\title{
Optimal Mode Localization in Disordered, Periodic Structures

\begin{tabular}{c|c|} 
by & LIBRARY \\
\cline { 2 - 2 } Gopalkrishna Rajagopal & $\begin{array}{c}\text { woons HoL, HASS. } \\
\text { W. H. O. i. }\end{array}$ \\
\hline
\end{tabular}

Bachelor of Technology, Indian Institute of Technology Madras

Submitted to the

Department of Ocean Engineering, MIT

and the

Department of Applied Ocean Physics and Engineering, WHOI

in partial fulfillment of the requirements for the degree of

Doctor of Philosophy in Oceanographic Engineering

at the

\section{MASSACHUSETTS INSTITUTE OF TECHNOLOGY \\ and the}

\section{WOODS HOLE OCEANOGRAPHIC INSTITUTION}

February 1995

(c) Gopalkrishna Rajagopal. All rights reserved.

The author hereby grants to MIT and WHOI permission to reproduce and to distribute publicly paper and electronic copies of this thesis document in whole or in part.

Author
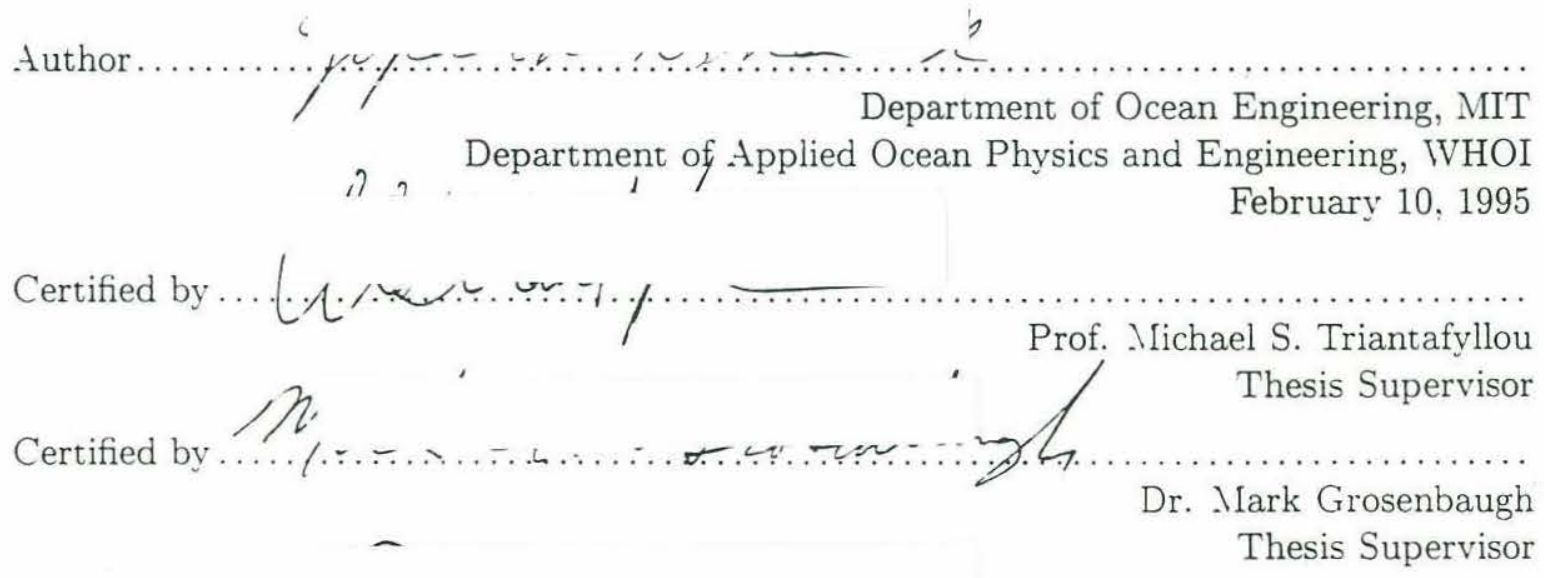

Accepted by<smiles>CCCC=CC=CC=CCC#CCCCCCC</smiles><smiles>C1#CCC1</smiles> 


\title{
Optimal Mode Localization in Disordered, Periodic
}

\section{Structures}

\author{
by \\ Gopalkrishna Rajagopal
}

\author{
Submitted to the Department of Ocean Engineering, MIT \\ and the \\ Department of Applied Ocean Physics and Engineering, WHOI \\ on February 10, 1995, in partial fulfillment of the \\ requirements for the degree of \\ Doctor of Philosophy in Oceanographic Engineering
}

\begin{abstract}
Periodic structures which are slightly disordered undergo dramatic changes in mode shapes such that the responses go from being spatially extended to spatially localized. This phenomenon called mode localization, offers an excellent option for passive vibration isolation.

In the first part of the thesis, we provide analytical prediction of modes exhibiting moderate localization using a newly developed Jordan Block Perturbation Method. We estimate and compare convergence zones of our newly developed method with perturbation techniques used to describe localized modes.

In the second part of the thesis, we provide numerical evidence that complex branch points, which occur for complex disorder values in the mode-disorder relation, are responsible for modal sensitivity. We investigate the effects of the strength of the branch point and their location in the complex plane.

In the third part of the thesis we perform an optimization study involving the selection of parameters which ensure a minimum level of localization of all modes. Optimal solutions were found to lie at maximum distances from the branch points, and the convergence basin of each optimum was demarcated by the branch point surface. The number of local optima were found to grow exponentially with the number of pendula. A statistical analysis showed that sampling of $10 \%$ provided an estimate that was within $2 \%$ of the global optimum, thereby reducing the computational effort for small to moderate systems of pendula. For larger systems of pendula, the problem of obtaining the global optimum in reasonable time still remains an open problem.

In the fourth part of the thesis we propose an application for mode localization in vibration isolation. An oceanographic mooring with regularly spaced buoys is investigated for localization of inline elastic oscillations. Localization is found to be useful for confining the harmonics in deep water moorings of $1000-4000 \mathrm{~m}$.
\end{abstract}

Thesis Supervisor: Prof. Michael S. Triantafyllou

Thesis Supervisor: Dr. Mark Grosenbaugh 


\section{Dedicated to Achan and Amma}




\section{Acknowledgements}

I first wish to thank the members of my committee, Professor Triantafyllou, Dr. Grosenbaugh and Prof. Yue for their help in this thesis. They were always full of ideas and suggestions and steered the thesis by their firm grasp of where the research fitted into the bigger picture. They were very approachable and fulfilled very admirably the complex tasks which go into being a teacher i.e. "friend, philosopher and guide". Special thanks to Dr. Dana Yoerger for his help in the late stages of the thesis.

I wish to gratefully acknowledge educational support of the Office of Naval Research contract numbers N00014-89-C-0179 and N00014-90-C-0098. Computational time was supported by Office of Naval Research under grant number N00014-92-J1269.

I wish to thank Mike Drooker of the Design Laboratory at MIT and Marty Marra of the Deep Submergence Laboratory at WHOI for their help on the computers at both places. I wish to thank everyone at Headquarters at MIT and Education at W.H.O.I for all the work behind the scenes.

I have been fortunate in having a group of bright and enthusiastic set of friends and colleagues who made a big impact on this thesis. Thanks are due to Drs. Chris, Franz, Knut and Ram and Drs-to-be Dave, Jamie and Thanassis for always being willing to give a helping hand, for discussing problems in research and for providing a fun atmosphere to work in.

I wish to thank Yuming for discussions regarding applications of localization in the water wave problem. I wish to thank Chick, Joe, Tarun and all the others from the other side of the office for maintaining a lively ambience in the office.

I wish to thank my room-mates over the years Dinesh, Sumanth, Tom and Venkat for all those long philosophical discussions and fun times together. There are many other friends who have been very helpful over the years at MIT and WHOI. I wish to place on record my thanks to them - I wish I had more space to list all those names.

Finally I wish to thank my parents for their patience, consideration and support while I finished my graduate studies. 


\section{Contents}

1 Introduction $\quad 15$

1.1 Motivation for Thesis . . . . . . . . . . . . . . 16

1.2 History Of Localization . . . . . . . . . . . . . . . . 17

1.3 Review of Work by Triantafyllou and Triantafyllou . . . . . . . 23

1.3.1 Localization: The problem and the need for a more mature understanding of the subject . . . . . . . . . . . 23

1.3.2 Main Points of Geometric Theory . . . . . . . . . . . . . 24

1.4 Goals and Contributions of Thesis . . . . . . . . . . . . . . 27

1.5 Outline of Thesis . . . . . . . . . . . . . . . . . . . . 29

2 Analytical Prediction of Localized Modes using Perturbation Techniques 31

2.1 Introduction . . . . . . . . . . . . . . . . 31

2.2 Two pendula problem $\ldots \ldots \ldots \ldots \ldots \ldots \ldots \ldots \ldots$

2.3 Procedure for $n$-order Jordan block expansion _. . . . . . . . . 39

2.3.1 Application of Jordan Block Expansion to Two Pendula . . . 48

2.4 Higher Order Systems . . . . . . . . . . . . . . . . . . 54

2.4.1 Prediction of modes for ten pendula using lower order coales-

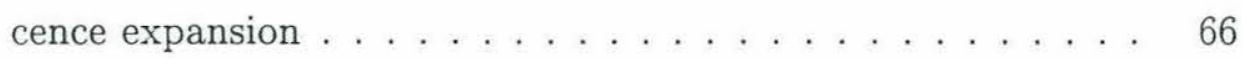

2.5 Conclusions . . . . . . . . . . . . . . . . . . . . . . 69

3 Investigation of the effects of the strength and location of the branch $\begin{array}{ll}\text { points on localization } & 71\end{array}$ 
3.1 Introduction . . . . . . . . . . . . . . 71

3.2 Two pendula example . . . . . . . . . . . . . . . . . . . 73

3.3 Three Pendula Example . . . . . . . . . . . . . . . . 76

3.4 Optimal directions to maximize localization . . . . . . . . . . . . 93

3.5 Quantifying difference between two and three root coalescences. . . . 95

3.6 Conflicting effects of strength and distance from the real axis : Trends and Examples . . . . . . . . . . . . . . . . . . . . 97

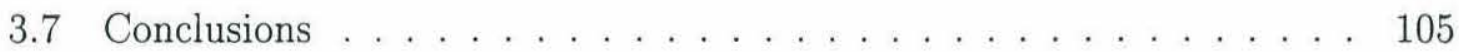

4 Optimal Mode Localization $\quad 106$

4.1 Introduction . . . . . . . . . . . . . . . . . 106

4.2 Work done in this chapter . . . . . . . . . . . . . . 107

4.3 Statement of the Problem . . . . . . . . . . . . 108

4.3.1 Problem 1 :Minimum Disorder to attain Minimum Level of Localization . . . . . . . . . . . . . . . . 108

4.3.2 Problem 2: Maximize Localization for given Mean Disorder . . 108

4.3.3 Problem 1: Objective Function . . . . . . . . . . . . 109

4.3.4 Problem 2: Objective Function . . . . . . . . . . . . . . . . . 110

4.4 Distribution of Optimal Solutions . . . . . . . . . . . . . . . 110

4.5 Development of an Algorithm to determine the Global Minimum . . . 115

4.6 Discussion of the performance of the algorithm . . . . . . . . . . 125

4.6.1 Operation Count . . . . . . . . . . . . . 127

4.7 Statistical Analysis of the Distribution of Optima . . . . . . . . . . . 129

4.7.1 The Weibull Distribution . . . . . . . . . . . . . . . . . . 134

4.8 Optimal solutions for larger systems of pendula . . . . . . . . . . . 141

4.9 Conclusions . . . . . . . . . . . . . . . . . . . . . . . . . . . . . 148

5 Mode Localization as a passive vibration isolation device in a real$\begin{array}{ll}\text { world structure } & 150\end{array}$

5.1 Introduction . . . . . . . . . . . . . . . . . 150

5.2 Work done in this chapter . . . . . . . . . . . . . . 151 
5.3 Equations of Motion . . . . . . . . . . . . . . . . . . . . 151

5.4 Modes of Vibration of Periodic and Disordered Structure . . . . . 152

5.5 Conclusions . . . . . . . . . . . . . . . . . . . . . . . . 169

6 Conclusions and Recommendations for Future Work 171

6.1 Main Features . . . . . . . . . . . . . . . . . . . . . . . . . . . . . . 171

6.2 Summary of Conclusions . . . . . . . . . . . . . . . . 172

6.3 Future Work . . . . . . . . . . . . . . . . . . . . . . . 174

A Definition of Modal Sensitivity and Localization Factor 175

A.1 Definitions of Modal Sensitivity Parameter and Localization Factor . 175

B Solutions to Jordan Block Size Three Perturbation 179

C Examples of Branch Point Surfaces and their effects on Localization 183

$\begin{array}{ll}\text { D Solution of the System of Equations } & 188\end{array}$

D.0.1 The General Form of Equations . . . . . . . . . . . . . . 189

D.0.2 Method of Steepest Descent . . . . . . . . . . . . 190

D.0.3 Model-Trust Region Approaches . . . . . . . . . . . . . . . 193

D.0.4 Comparison Between the Different Methods . . . . . . . . 194 


\section{List of Figures}

1-1 Eigenvalues as a function of disorder. Case (a) : Independent eigenvalues. Case (b) : Coalescent Eigenvalues. . . . . . . . . . . 25

2-1 A System of Coupled Pendula . . . . . . . . . . . . . . 32

$2-2$ System of Two Pendula . . . . . . . . . . . . . . . 34

2-3 Comparison of convergences of Jordan, MPM and CPM Methods. - : Exact. --: Perturbation prediction. . . . . . . . . . . 38

2-4 Comparison of errors in eigenvector predictions of the Jordan, CPM and $\mathrm{MPM} \ldots \ldots \ldots \ldots$

$2-5$ System of three pendula . . . . . . . . . . . . . . 55

2-6 -: Two root coalescences. (a,b): Projection of three root coalescence

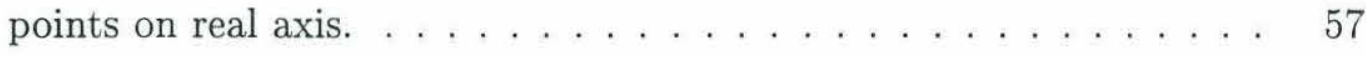

2-7 (a) : Jordan block and MPM predictions at $P 1\left(\epsilon_{1}=.01, \epsilon_{2}=\right.$ .01) Branch point (a) Jordan block expansion. (b) : Jordan block and MPM predictions at $P 2\left(\epsilon_{1}=.01, \epsilon_{2}=-.01\right)$ Branch point (b) Jordan block expansion, (c) : Jordan block and MPM predictions at $P 3\left(\epsilon_{1}=.06, \epsilon_{2}=-.06\right)$. ( $\square$ : Exact Eigenvector, $\diamond$ : Predicted Eigenvector.)

2-8 (a) : Jordan block and MPM predictions at $\left(P 1^{\prime}\right) \epsilon_{1}=0.06, \epsilon_{2}=$ .001. Expansion about square-root branch point at $\left(\epsilon_{1}=0.06, \epsilon_{2}=\right.$ $0.0001828+.004099 i)$. (b) : Jordan block and MPM predictions at $\left(P 2^{\prime}\right) \epsilon_{1}=.06, \epsilon_{2}=.03$ ( $\square$ : Exact Eigenvector, $\diamond$ : Predicted Eigenvector.) 
2-9 Convergence zone for MPM and envelope of convergence for Jordan block expansion . . . . . . . . . . . . . . . . . 6 63

2-10 (a) : Jordan block (a) and Jordan block (b) predictions at $B 1\left(\epsilon_{1}=.005, \epsilon_{2}=.01\right)$. (b) : Jordan block (a) and Jordan block (b) predictions at $B 2\left(\epsilon_{1}=.005, \epsilon_{2}=-.01\right)(\square$ : Exact

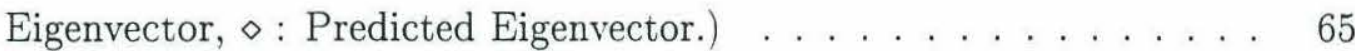

2-11 Jordan Block expansion (a) and CPM (b) predictions for ten pendula model at $\epsilon_{9}=-.2643$. Jordan block expansion (c) and CPM (d) expansion at $\epsilon_{9}=.0043 . \square$ : Exact Value, $\diamond$ : Predicted Value. . . . . 70

3-1 Modal Sensitivity as a Function of Disorder for $R^{2}=.01, R^{2}=.05$,

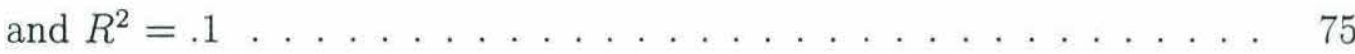

3-2 Position of branch point versus disorder . . . . . . . . 77

3-3 Localization factor versus disorder . . . . . . . . . . . . . 78



3-5 Gradual transition from cube-root branch point to square root branch point. Point P1 : $\epsilon_{1}=.0043, \epsilon_{2}=-.01186$. (a) : Jordan block size three expansion about (a). Jordan block size two expansion about $\epsilon_{1}=.0043, \epsilon_{2}=-.01186+.01616 i$. Point P2 : $\epsilon_{1}=.02, \epsilon_{2}=-.003335576$. (b) : Jordan block size three expansion about (a). Jordan block size two expansion about $\epsilon_{1}=.02, \epsilon_{2}=$ $-.003335576+.0112921 i$. Point P3 $: \epsilon_{1}=.06, \epsilon_{2}=-.00018283$. (c) : Jordan block size three expansion about (a). Jordan block size two expansion about $\epsilon_{1}=.06, \epsilon_{2}=-.00018283+.004099 i$. ( $\square$ : Exact Eigenvector, $\diamond$ : Predicted Eigenvector.) _ . . . . . . . . . 81

3-6 Variation of magnitude of $\operatorname{Imag}\left(\epsilon_{1}\right)$ with $\epsilon_{2}$ for square-root branch point curve. ०: Imaginary part of projection of cube-root branch point . . . 82

3-7 Schematic diagram of branch point surfaces for two parameter system 84 3-8 (a) : $\operatorname{Real}\left(\epsilon_{2}\right)$ versus Imaginary $\left(\epsilon_{1}\right)$. (b) $: \operatorname{Imag}\left(\epsilon_{2}\right)$ versus $\operatorname{Imaginary}\left(\epsilon_{1}\right) 85$

3-9 Modulus of modal sensitivity : $Q\left(q_{i}\right), i=1,2,3 \ldots \ldots \ldots 8$ 
3-10 Localization factor contours for three pendula. $\star$ : Projection of cuberoot branch points on real disorder plane. . . . . . . . . . . . . 89

3-11 Figures (a-c): Mode Shapes at (a), $\epsilon_{1}=-.04, \epsilon_{2}=-.03$. a-c $:$ Jordan block size two expansion about $\epsilon_{1}=-.03, \epsilon_{2}=-.042+.018 i$, d-f : MPM expansion. Figures (d-f): Mode Shapes at (b), $\epsilon_{1}=0.05, \epsilon_{2}=0$. Jordan block size two expansion about $-0.0003+0.005 i$. . . . . . 90

3-12 Mode Shapes (a-c): $\epsilon_{1}=-.48, \epsilon_{2}=-.428$, Mode Shapes(d-f) $: \epsilon_{1}=0$, $\epsilon_{2}=-.0152$, Mode Shapes $(\mathrm{g}-\mathrm{i}): \epsilon_{1}=0, \epsilon_{2}=.49$, Mode Shapes $(\mathrm{j}-\mathrm{l})$ $: \epsilon_{1}=-.48, \epsilon_{2}=0$, Mode Shapes (m-o) $: \epsilon_{1}=.003, \epsilon_{2}=0$ Mode Shapes $(\mathrm{p}-\mathrm{r}): \epsilon_{1}=.49, \epsilon_{2}=.4675 \ldots \ldots \ldots \ldots 2$

3-13 Optimal Search Directions to maximize localization superposed on localization factor contours for mode 2: '-' : Directions a1-a6. . . . . . 94

3-14 (a) : Jordan block size two expansion about $\epsilon_{1}=0, \epsilon_{2}=-.0152+$ .0169i. (b) : Jordan block size three expansion about branch point (a). (c) : Jordan block size three expansion about branch point (b). (d) : Jordan block size two expansion about $\epsilon_{1}=0, \epsilon_{2}=.0030-.0113 i \ldots 96$

3-15 Six Pendula System : Modes close to Six Root Coalescence . . . . . . 98

3-16 Six Pendula System : Modes close to Three Root Coalescences . . . . 99

3-17 Six Pendula System : Modal Sensitivity for six root coalescence : $Q\left(q_{i}, \epsilon_{5}\right)$ where $1 \leq i \leq n . \ldots \ldots \ldots \ldots \ldots$

3-18 Modal Sensitivity for the three root coalescences. Group (a) : $Q\left(q_{i}, \epsilon_{0}\right)$ and Group (b) : $Q\left(q_{i}, \epsilon_{5}\right)$ where $1 \leq i \leq n \ldots \ldots \ldots \ldots$

3-19 Modal Sensitivity for the three root coalescences. Group (a): $Q\left(q_{i}, \epsilon_{0}\right)$ and Group (b): $Q\left(q_{i}, \epsilon_{5}\right)$, where $1 \leq i \leq n \ldots \ldots \ldots . \ldots 103$

4-1 Distribution of Local Optima for System of Three Pendula : Objective Function $1 \ldots \ldots \ldots \ldots \ldots \ldots 111 \ldots \ldots \ldots$

4-2 Distribution of Local Optima for System of Three Pendula : Objective Function $2 \ldots \ldots \ldots \ldots$

4-3 Convergence Basin for Steepest Descent Method for Three Pendula . 114 
4-4 Examples of legal and illegal search directions f . . . . . . . . 117

4-5 Distribution of Local Optima for System of Four Pendula . . . . . . 118

4-6 Modes at a1 for four pendula system . . . . . . . . . . . . . . . 119

4-7 Modes at a2 for four pendula system . . . . . . . . . . 120

4-8 Modes at a3 for four pendula system . . . . . . . . . . . . . 121

4-9 Modes at a4 for four pendula system . . . . . . . . . . . 122

4-10 Modes at global optimum of six pendulum system, $\epsilon_{5}=.0430, \epsilon_{4}=$ $-.0486, \epsilon_{3}=-.0917, \epsilon_{2}=-.0785, \epsilon_{1}=.0743 \ldots \ldots \ldots \ldots 124$

4-11 Plot of Variation of Number of Optima with Number of Pendula . . . 126

4-12 RMS disorder distribution for local optima for six pendula system . . 130

4-13 RMS disorder distribution for local optima for three pendula system . 132

4-14 RMS disorder distribution for local optima for four pendula system . 133

4-15 RMS data plot on Weibull graph without cutoff . . . . . . . 135

4-16 RMS data plot on Weibull graph with cutoff . . . . . . . . . 136

4-17 Fitted Weibull Distribution . . . . . . . . . . . . . 138

4-18 Minimum of sample(expressed as a percentage of global minimum) vs. sample size.-: Confidence Bound from Weibull Distribution. o: Actual Minimum. . . . . . . . . . . . . . . . . . 140

4-19 Modes of ten pendulum for objective function 1: $\epsilon_{9}=.2201, \epsilon_{8}=$ $.1029, \epsilon_{7}=.0434, \epsilon_{6}=.1599, \epsilon_{5}=.0211, \epsilon_{4}=.1992, \epsilon_{3}=.1992, \epsilon_{2}=$ $.0797, \epsilon_{1}=.2299$, RMS disorder $=.1436 \ldots \ldots \ldots \ldots \ldots$

4-20 Modes of ten pendulum system for ten pendula for objective function $2: \epsilon_{9}=.0613, \epsilon_{8}=.0101, \epsilon_{7}=-.0296, \epsilon_{6}=-.0554, \epsilon_{5}=.0519, \epsilon_{4}=$ $-.0149, \epsilon_{3}=-.0431, \epsilon 2=-.0698, \epsilon_{1}=.0375$, RMS disorder $=.05 \quad \ldots 143$

4-21 Localization factor as a function of end pendulum disorder for three and thirty pendula system . . . . . . . . . . . . . . 144

4-22 Log of absolute amplitude of mode shape of thirty pendula system . . 144

4-23 An Optimal Solution for the Eight Pendula Problem : Two groups of four root coalescences . . . . . . . . . . . . . . . . 146 
4-24 An Optimal Solution for the Eight Pendula Problem : Four groups of two root coalescences . . . . . . . . . . . . . . . . . . . . . . . 147

5-1 Schematic Diagram of Oceanographic Mooring . . . . . . . . . . 153

5-2 Free Body Diagram of Parts of Mooring. $u_{i}$ : Displacement of $i$ th segment. $M$ : Virtual Mass of buoy. $s$ : Coordinate along cable. $D$ : Drag on buoy. $E$ : Youngs modulus of the cable. $A$ : Area of cable.



5-3 Fundamental Set of Modes for Periodic Structure . . . . . . . . 156

5-4 First Harmonic Set of Modes for Periodic Structure . . . . . . . 157

5-5 Second Harmonic Set of Modes for Periodic Structure . . . . . . . . . 158

5-6 Fundamental Set of Modes for Disordered Structure . . . . . . . . 160

5-7 First Harmonic Set of Modes for Disordered Structure . . . . . . . . . 161

5-8 Second Harmonic Set of Modes for Disordered Structure . . . . . . 162

5-9 Pierson Moskowitz Spectrum . . . . . . . . . . . . . . . . . . . 164

5-10 Design Curves for selection of $E$ and the distance between masses . . 165

5-11 Transfer Function for Periodic Case . . . . . . . . . . . . . . 167

5-12 Transfer Function for Disordered Case . . . . . . . . . . . 168

5-13 Transfer Function for Disordered Case for Boundary Condition 2 . . . 170

6-1 Flow of Research in Mode Localization . . . . . . . . . . . . . . 173

A-1 Example where the exponential fit is good. $\epsilon_{1}=.1, \epsilon_{2}=-.15 \quad \ldots \quad 177$

A-2 Example where the exponential fit is poor. $\epsilon_{1}=.005, \epsilon_{2}=.1 \ldots 178$

C-1 Modal Sensitivity Parameter $Q\left(q_{j}, \epsilon_{1}\right) \ldots \ldots \ldots \ldots \ldots$

C-2 Four Pendula System : Two Root Coalescence Surface Number 1

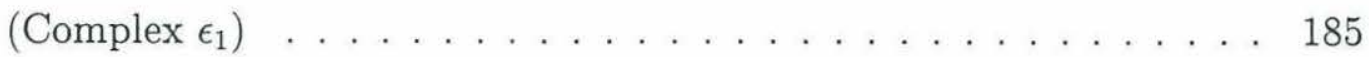

C-3 Four Pendula System : Two Root Coalescence Surface Number 2

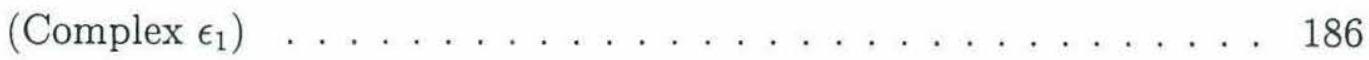

C-4 Four Pendula System : Two Root Coalescence Surface Number 3

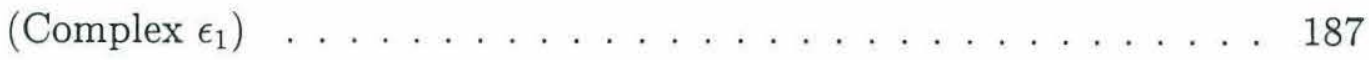


D-1 Example Case where the Steepest Descent Method is inefficient . . . 192 


\section{List of Tables}

2.1 Three Root Coalescences . . . . . . . . . . . . . . . . 56

3.1 Three Root Coalescences . . . . . . . . . . . . . . . . 86

3.2 Six Root Coalescences . . . . . . . . . . . . . . . . . . . . . . . 97

3.3 Three Root Coalescence for configuration 1 . . . . . . . . . . . . . 101

3.4 Three Root Coalescence . . . . . . . . . . . . . . . . . . 104

4.1 Coordinates of points a1,a2,a3 and $\mathrm{a} 4 \ldots \ldots . . . \ldots 123$

5.1 Disorder . . . . . . . . . . . . . . . . . . 155

5.2 Column 1 : Cable Parameters, Column2 : Mass Parameters . . . . . 155

5.3 Column 1: Cable Parameters, Column2 : Mass Parameters . . . . . 166 


\section{Chapter 1}

\section{Introduction}

Elastic, periodic structures are characterized by spatially extended mode shapes and responses to input forcings (See Brillouin [6]). The typical periodic structure met in engineering practice can be modeled as a system of oscillators with identical natural frequencies. These are coupled together by some appropriate coupling element to build up the periodic structure.

Under conditions of weak coupling, small changes in the periodicity (disorder) result in very dramatic changes in the dynamics of the system. Disordered, periodic structures are characterized by spatially localized mode shapes and responses to input

forcings even at resonance. Thus, small perturbations to the structure have resulted in dramatic changes to the response and mode shapes of the system. Since the response of the system is uniquely determined by the modes of the system, it is evident that the key to understanding localization lies in understanding the sensitivity of the mode shapes to perturbations.

The remarkable feature about localization is that conservative systems with a minimal amount of damping display confinement of vibration about the driving point. Damping is unimportant in this phenomenon except as a means of preventing catastrophic failure by draining out energy during steady state excitation of the structure. So damping can be ignored during analysis of localization. 


\subsection{Motivation for Thesis}

We will be examining the dynamics of disordered, periodic structures. We will, for most of this thesis, restrict our attention to a system of identical coupled pendula or coupled oscillators because this system is sufficiently simple to permit analytical treatment of the system while capturing all features of the dynamics of more complicated periodic structures. This has become a canonical system in the study of localization.

The main feature of localization is the extreme sensitivity of the mode shapes of the structure to small perturbations of the periodicity of the structure. This sensitivity has a number of features on which we will comment.

Previous authors like Cornwell and Bendiksen [9] have pointed out that if we view the modes as a continuous function of the disorder, the modes make the transition from extended to localized over a very narrow range of disorder. In other words, the localization is not a linear function of disorder. In general as disorder is input into the structure the modes change very dramatically initially, and then as we reach larger values of disorder the change is very little even though we increase disorder substantially.

The structure appears to be sensitive to the precise combination of disorder input into the structure. For example, if we increase the natural frequencies of all the oscillators by the same amount, we still have a periodic structure and periodic mode shapes. If we increase the natural frequencies of one of the pendula only, to be much greater than the rest, its dynamics becomes decoupled from those of the remaining pendula because of the large difference in natural frequencies, and intuitively we can expect one mode to be significantly localized about that pendulum. It is thus obvious that the modes display different levels of sensitivity and localization depending on the combination of disorder input into the system and the actual functional dependence of the mode shapes on the disorder can be very complex. This fact can be seen in the results from extensive numerical experiments conducted on a system of coupled oscillators by Hodges and Woodhouse [19]. Any theoretical attempts to understand 
localization must be able to explain all of the varied aspects of this sensitivity of the mode shapes.

The reasons for interest in this sensitivity are twofold. The first, is the academic reason of understanding localization. The second, is the tremendous potential that localization offers as a passive vibration isolation device in ocean structures. It is difficult to apply conventional vibration isolation methods (using the presence of anti-resonances in the transfer function) to these structures because the resonances are closely spaced and narrow banded excitation would still excite all the modes. Localization is a viable option because even when we excite the structure at resonance, we still have a response confined about the excitation point. During steady-state excitation of the structure we have a buildup of energy in the structure. During localization, damping permits the structure to reach a steady-state by draining out excess energy in the structure.

In sum, the two main reasons which motivated this thesis were the need to understand the large modal sensitivity in structures whose modes can be localized and the need to introduce disorder to ensure passive vibration isolation while ensuring that drag is minimum.

\subsection{History Of Localization}

Localization was first predicted by Anderson [1] in the context of solid state physics. This was first described in the context of the eigenstate localization of an electron in a three dimensional lattice. The existence of localization in one dimensional lattices was first shown by Borland [5].

Structural applications of localization deal with the one dimensional lattice. Its

occurrence in structural dynamics was first shown by Hodges [16]. It must be pointed out here that most of the localization seen in solid state applications is for periodic structures where the substructures are of the order of 50 to 100 at least. The structural dynamics applications on the other hand deal with a far smaller number of substructures, typically, less than twenty. 
Early, fundamental work on localization in engineering structures was done by Hodges [16]. He demonstrated the existence of localization for short wavelength waves propagating in a structure. This would correspond to acoustic waves. Hodges and Woodhouse ([19]) examined structural applications by doing extensive numerical studies for systems of coupled oscillators and provided penetrating physical descriptions of the problem. They have provided insights into the statistical properties of the response of a system of oscillators when subject to input forcing. In particular, they have demonstrated how the logarithm of the response of the disordered structure, when averaged over many realizations of the ensemble containing all possible combinations of disorder that could be input into the system, yields a well defined mean. This has been used as the basis of the definition of measures for the localization in the system by other authors like Kissel [20] and Pierre [29].

An excellent review of localization is provided by Hodges and Woodhouse [18]. Here, they explained the equivalence of the modal and traveling wave formulation for vibrations in structures. They also discussed the connection with other commonly used analytical tools like Statistical Energy Analysis (SEA). They performed experiments to prove the existence of localization in a system of masses on a string [17]. This was the first experimental demonstration of localization in a structure.

Since system responses are uniquely determined by the free modes of vibration of the system, many studies of localization using perturbation techniques applied to the eigenvalues and modeshapes of the system were carried out. Perturbation studies were done by Pierre and Dowell [27], and Pierre and Cha [30]. They identified the fact that the two broad parameters affecting the problem were the coupling and the disorder. In general, if the disorder was larger than the coupling the modes looked strongly localized while if the coupling was larger than the disorder, the modes looked weakly localized. Small perturbations about the periodic state were described by the Classical Perturbation Method (CPM) which used the disorder as the expansion parameter in the perturbation expansion. The unperturbed state would comprise a set of spatially extended mode shapes. This method however failed to provide effective prediction of strongly localized mode shapes. Such mode shapes violated the 
assumption that the coupling was stronger than the disorder. Pierre and Dowell ([27]) proposed an alternative scheme called the Modified Perturbation Method (MPM) where the coupling was treated as the expansion parameter and the unperturbed state was the localized state. This has proved to be effective in the analytical prediction of strongly localized mode shapes. Such mode shapes have large amplitudes over one oscillator and have a small nonzero amplitude over a few others.

The analytical prediction of moderately localized modes has still remained an open issue as has been pointed out by Cornwell and Bendiksen [9]. Some attempts have been made to address this problem by Happawana et al. [15] who attempted to use singular perturbation methods to predict eigenvalues corresponding to a state of moderate localization. This singular perturbation was applied about the uncoupled disordered state. Two criticisms can be levelled at the approach they took. The method is very cumbersome for even a small system of two coupled pendula. The second criticism is that the method obscures a lot of the physics involved in the problem. This harks back to some of the issues raised by Pierre and Dowell ([27]) in another context involving matrix perturbations about the uncoupled, periodic state where physical understanding can be sacrificed for accuracy of prediction by using such a state as the unperturbed state for performing perturbation calculations. Pierre and Dowell ([27]) discarded matrix perturbation expansions about the uncoupled periodic state because such a perturbation expansion did not provide any new information about the system eren though it might have provided accurate predictions of the eigenvalues and eigenvectors. This is true in this case also. Both methods are very unwieldy and require considerable amounts of complicated algebra. The authors have attributed the rapid change of eigenvectors to the singular point about which the singular perturbation was performed. This may be wrong. This would imply the point of maximum sensitivity is at the state of zero disorder and that may not be correct. They examined cases of very weak coupling and hence the modal sensitivity plots they show have maximum sensitivity at zero disorder. We will show in this thesis that the singularity responsible for the sensitive behavior of the eigenvectors is a branch point type singularity and the peak modal sensitivity does not necessarily occur at the 
state of zero disorder, especially for cases involving moderate coupling. The authors also seem, not to have provided any predictions of mode shapes using their singular perturbation techniques which is after all more critical given that we are studying "mode localization".

Cornwell and Bendiksen [10], Valero and Bendiksen [40] have investigated the existence of localization in another type of structure, the dish antenna. This is a system where we have a periodicity of a different kind. We have a rotary structure with the $n$th and first oscillators being connected to each other. In addition to dish antenna, they are important as models while studying turbine rotors and propellers. These authors have also done some extensive parametric studies on the problem where they noted that the transition of modes from extended to localized state occurs rapidly over a small range of parameters. They however could not identify the precise cause of the transition from extended to localized state.

Additional aspects of localization have included association with the phenomenon of curve veering. In certain systems, eigenvalue loci of the system, when plotted as a function of a system parameter (for the system of coupled pendula, it is the disorder) approach each other and then rapidly veer away with interchange of mode shapes. This phenomenon is called curve veering. Pierre [28] found that the eigenvalue loci of the system of coupled pendula, a system which displayed mode localization, also exhibited curve-veering. He used conditions for curve-veering to occur (Perkins and Mote [25]) and showed that the conditions for localization to occur and those for curve-veering to occur are both linked to the existence of weak coupling.

Much of the motivation for this thesis comes from the study by Triantafyllou and Triantafyllou [39] where localization was studied from a geometric standpoint. Existing studies, using perturbation techniques, indicated that the main cause of the large sensitivity of mode shapes seen during localization was due to the existence of closely spaced eigenvalues as seen in a system of coupled pendula. Triantafyllou and Triantafyllou pointed out it was misleading to attribute the large sensitivity seen in such systems to closely spaced eigenvalues. The central features of localization and associated curve veering were shown to be associated with the existence of branch 
points in the frequency-disorder relation using asymptotic expansions. The branch points were shown to be linked to the existence of eigenvalue coalescences. In general, for a system with $n$ eigenvalues, we could have $n$th root dependence of the eigenvalue on the system parameters, which would be linked to the existence of coalescences of $n$ eigenvalues occurring for complex values of the parameter. The non-analytic nature of the branch point was held responsible for the dramatic changes in mode shapes which occurred for small perturbations applied to the system. Triantafyllou and Triantafyllou also showed that these branch points are responsible for the twin phenomena of mode localization and curve veering.

This thesis does not cover all aspects of localization. However for completeness sake, we will review other work that has been performed in localization studies.

Kissel [20] investigated the problem statistically, drawing on work performed by Hodges and Woodhouse ([19]) and solid state physics to define a localization factor associated with the localized transmitted wave in a disordered structure. He calculated the localization factors associated with transmitted waves in various periodic structures averaged over many realizations from an ensemble of disorder. This decay factor was frequency dependent and he systematically created many frequency dependent plots of the localization factor for disorder drawn from uniform probability distributions with different standard deviations, for a variety of systems which would model engineering structures met in the real world. A big criticism levelled by Pierre [29] was that the structures examined by Kissel [20] did not allow for the existence of strong localization because he did not examine structures with internal coupling.

Pierre [29] utilized statistical perturbation methods to compare those predictions with the results of Monte Carlo simulations of the type done by Kissel, but for structures with internal coupling to allow for the existence of strong localization. He found that it was not possible to correlate the perturbation and Monte Carlo predictions for modes in a state of moderate localization. The Monte-Carlo and perturbation predictions for weakly and heavily localized modes were in excellent agreement. Seides [37] also performed such calculations with emphasis on marine structures. The statistical study of localization, while being a very interesting subject in itself, is not being pur- 
sued in this thesis. We will be focusing exclusively on the effects of deterministically introduced disorder.

Balmes [2] has provided some interesting observations about systems with high modal density i.e. systems where the modal damping is larger than the separation between natural frequencies. He performed some numerical simulations to demonstrate cases where the mode shapes are very sensitive to small amounts of disorder, but the frequency response of the system remains relatively unaffected by the disorder.

Experimental investigation of localization started with the fundamental work by Hodges and Woodhouse [17]. This was followed up with work by Pierre and Cha [30] and Levine and Salama [22]. They looked at localization seen in multispan coupled beams and in a space reflector respectively. Rajagopal ([34]) had conducted some experiments to satisfy ourselves about the localization process. We examined a structure similar to that examined by Hodges and Woodhouse, although we were examining it using steady state excitation. We did find localization achievable in this structure.

Most of the studies reviewed so far have tended to idealize engineering structures as discrete coupled oscillators. Very interesting work on continuous systems has been done by Luongo [23] where he considered the longitudinal free oscillations of a beam with small axial rigidity continuously restrained by imperfect elastic springs. He showed that the problem can be viewed as being governed by a turning point problem. Some asymptotic predictions using WKB methods were obtained. Another very interesting piece of research was done by Devillard, Dunlop, and Souillard [12] where they examined gravity waves in a one-dimensional channel. Localization was studied for a bottom with a series of random rectangular steps. Transfer matrices for the linear dynamics of water waves on a flat shelf were used to model the dynamics of the system. Experimental evidence of localization for the water wave problem was provided by Belzons et al. 


\subsection{Review of Work by Triantafyllou and Tri- antafyllou}

Since this thesis was motivated in large measure by the paper by Triantafyllou and Triantafyllou [39], we will make a detour to explain the concepts in that paper.

\subsubsection{Localization : The problem and the need for a more mature understanding of the subject}

Consider a system of identical coupled pendula. We now permit disorder to be intro-

duced into this periodic system. Each pendula can have a perturbation $\epsilon_{i}$ from the unperturbed state. For the sake of standardization of the problem. we will always examine a set of pendula with length 1 . The coupling between the pendula are also all identical and could be "weak" or "strong". The periodic system is characterized by a set of extended mode shapes. This is a result of Floquet theory and is explained in great detail in Brillouin [6]. Small alterations to the system (disorder) can result in dramatic changes in the mode shapes from a spatially extended state to a spatially localized state. It is found that the tendency for modes to be localized is more prevalent when the coupling is weak. Obviously, such rapid transition of mode shapes from extended to localized state implies extreme sensitivity of the modes. The cause of the extreme sensitivity has been understood to be caused by the "small denominator" effect. Classical perturbation studies have shown that large changes to the mode shapes resulting in change from extended to localized state are caused by the denominator of the coefficients of the perturbation series being very small (hence the name). The geometric theory however advances the cause of the large modal sensitivity seen during localization as due to something more fundamental, which we will explore in this section. Another intriguing aspect of localization is the fact that different combinations of disorder result in very different levels of localization of mode shapes in the system. We will see in this section that the geometric theory helps us understand the division of different regions of the parameter space into regions with 
more and less localization.

\subsubsection{Main Points of Geometric Theory}

There are three main stages in the development of the theory of Triantafyllou and Triantafyllou ([39]).

We start by examining the mapping defined by the characteristic polynomial of the eigenvalue problem associated with a system which exhibits localization like a system of coupled pendula. This is a mapping from the disorder parameter space to the eigenvalues. There are $n$ distinct eigenvalues which are obtained by solving the eigenvalue problem. For any given value of disorder, the eigenvalues may be distinct, or coalescent (See figure 1-1). The conditions for $n$ coalescent eigenvalues in a general $n$ parameter system ar

$$
\begin{gathered}
\Delta\left(\lambda, \epsilon_{1}, \ldots \epsilon_{n-1}\right)=0 \\
\frac{\partial^{i} \Delta}{\partial \lambda^{i}}\left(\epsilon_{1}, \ldots, \epsilon_{n-1}, \lambda\right)=0
\end{gathered}
$$

where $1 \leq i \leq(n-1)$. Here $\lambda$ is the eigenvalue and $\epsilon_{i}$ is the disorder parameter. These are conditions for a saddle point to exist. We have so far made the assumption that since we are studying a real system of coupled pendula, the disorder can only assume real values. The solution to the above system of equations, however may be complex. Triantafyllou and Triantafyllou [39] made the bold but perfectly admissible contention that we should permit the disorder parameters to become complex. We would thus be permitting the parameters by analytic continuity to assume complex values. We would be making the assumption that the real and imaginary parts of the mapping defined by the characteristic polynomial obey the Cauchy-Riemann equations. We are allowing for the existence of branch points, branch cuts and other such features in the complex plane.

The second stage of the analysis followed. Triantafyllou and by Triantafyllou ([39]) showed that these saddle points are associated with branch points in the frequencydisorder relation. The analysis used a Taylor expansion about a point at which the 

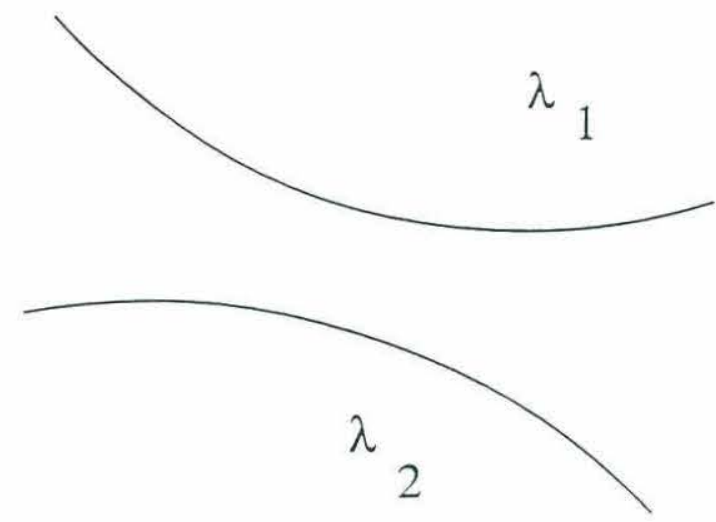

\section{(a) Distinct Eigenvalues}

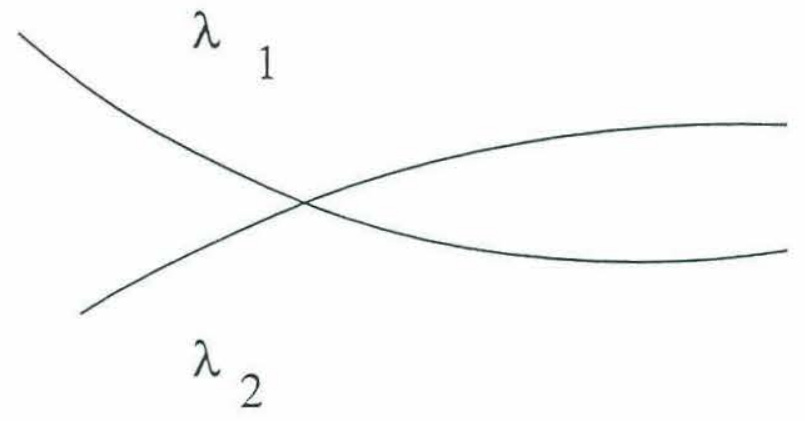

(b) Coalescent Eigenvalues

Figure 1-1: Eigenvalues as a function of disorder. Case (a) : Independent eigenvalues. Case (b) : Coalescent Eigenvalues. 
saddle point conditions for eigenvalue coalescence are satisfied. Consider a one disorder parameter system with disorder $\epsilon$. If we consider a saddle point and that point is denoted by the coordinates $\left(\lambda_{0}, \epsilon_{0}\right)$, the expansion for the characteristic polynomial is given by

$$
\Delta(\lambda, \epsilon)=\Delta\left(\lambda_{0}, \epsilon_{0}\right)+\frac{\partial \Delta}{\partial \lambda}\left(\lambda-\lambda_{0}\right)+\frac{\partial \Delta}{\partial \epsilon}\left(\epsilon-\epsilon_{0}\right)+\frac{\partial^{2} \Delta}{\partial \lambda^{2}}\left(\lambda-\lambda_{0}\right)^{2} \cdots
$$

At the point of eigenvalue coalescence.

$$
\begin{gathered}
\Delta\left(\lambda_{0}, \epsilon_{0}\right)=0 \\
\frac{\partial \Delta}{\partial \lambda}\left(\lambda_{0}, \epsilon_{0}\right)=0
\end{gathered}
$$

Using these two equations in the previous expansion for the characteristic polynomial, we collect the lowest order terms to get the following asymptotic relation

$$
\lambda=\lambda_{0}+B \sqrt{\left(\epsilon-\epsilon_{0}\right)}
$$

At such a point, the eigenvalues cannot be expanded in a Taylor series and a series in fractional powers of the disorder (a Pusieux series) only, can be used.

In general for an $n$ parameter system we could have any from two through $n$ root coalescences. Obviously an $n$th root coalescence is more desirable than a two root coalescence since the modes would be more sensitive (an nth root dependence) to small changes in disorder.

The third stage of the analysis was to point out that these complex coordinates of eigenvalue coalescence were also points where there was infinite eigenvalue sensitivity since we have branch points at these points. The stiffness matrix at those values of the complex coordinate were associated with Jordan Blocks of size greater than one. This implied that the associated eigenvectors would also be associated with 
infinite modal sensitivity. If the complex coordinate had a sufficiently small imaginary part, they would lie very close to the axis of real disorder. Evidently the eigenvalue sensitivity would increase if the imaginary part tended to zero. Hence Triantafyllou and Triantafyllou pointed out that any attempts to search for localization in structures should focus on looking for structures where the imaginary part of the complex branch point coordinate was as small as possible and the order of the coalescence was as large as possible.

They also pointed out that the failure of Pierre's perturbation schemes was directly related to the presence of these branch points. Triantafyllou and Triantafyllou however focused mainly on the eigenvalues of localizable systems and did not focus at all on the eigenvectors. They did not dwell at length on the modal sensitivity(as opposed to eigenvalue sensitivity) associated with these branch points. This is the starting point of this thesis. Modal sensitivity is the prerequisite for localization. It is important to link modal sensitivity with localization wherever possible. This was not done in Triantafyllou and Triantafyllou's paper. This is accomplished here. A complete investigation of the effects of the strength and location of branch points on localization is also performed.

\subsection{Goals and Contributions of Thesis}

The first contribution of this thesis is the development of Jordan Block perturbation methods to analytically describe modes in an intermediate state of localization. These modes are modes which display very high modal sensitivity and that makes for interesting study.

The second contribution is the outlining of a systematic procedure to determine the convergence zones of the various perturbation techniques.

The third contribution is providing numerical confirmation of the fact that branch points are directly responsible for the large modal sensitivity seen in systems which exhibit localization. This was done by numerical solution of the bifurcation equations provided by Triantafyllou and Triantayllou [39]. 
The fourth contribution is explaining the reason for the fact that different combinations of disorder with the same statistical characteristics result in different levels of localization (noted by Hodges and Woodhouse [19]). This was done by noting three facts which are obtained from the geometric theory. The first is that both the order of coalescence and magnitude of imaginary part of the complex branch point coordinate are responsible for modal sensitivity. The second is that the number of oscillators having significant modal amplitude is equal to the order of coalescence of the closest branch point. The third, is the number of modes having large modal sensitivity is directly related to the order of the coalescence of the closest branch point. These three facts can be used to explain the reason for Hodges and Woodhouse's results. Various conflicting effects of different order branch points and their implications on localization were explored. Specifically, the $n$th root sensitivity of modes implied that $n$ modes would display an $n$th root dependence on the disorder. Depending on the disorder combination we input, we could be close to branch points of different orders. A higher order branch point would cause more modes to have increased modal sensitivity as opposed to a lower order branch point if both were equally distant in the complex plane from real axis. The highest order branch point ( $n$ for an $n$ pendula system) was found to be fixed whereas the lower order branch points were found to form a surface with the imaginary part varying across the surface. Conflicts arose when the imaginary part of the lower order branch point was sufficiently small to cause the associated sensitivity to approach that of the higher order branch point. The mode shapes close to different order branch points were also found to be very different resulting in modes which were localized while appearing very different from each other. We also find a trend that for larger values of disorder, the lower order branch point is more important in affecting localization while for smaller values of disorder, the higher order branch points affect localization. The existence of optimal directions in the parameter space where localization is a maximum is also noted. The existence of a form of curve veering associated with the branch point loci is also noted.

The fifth contribution is the introduction of an algorithm using nonlinear optimization techniques to design a structure to ensure that all modes have a certain 
minimum level of localization while ensuring that the sum of the squares of the disorder is the minimum. The first result was that the optimal solution lay at the point of maximum distance from the two root coalescence branch point surfaces. The second result was the development of an algorithm to ensure that all the optima could be sequentially tracked down. The third result was that the number of optima and the computational effort increased exponentially with the number of pendula. The fourth result was a statistical analysis of these optima with relevance for smaller systems ranging from approximately two to ten pendula which indicated that sampling of a few of the optima, gave a good estimate of the global optimum. This vastly reduced the computer time taken given the implications of the third result. However the exponential growth of the optima with the number of pendula implied that obtaining a global optimum for a large system of coupled pendula in reasonable time still remained an open problem.

The sixth contribution was a real-life application of this method to an oceanographic mooring. The mooring was a taut cable with submerged buoys at regular intervals. The studies showed mode localization to be excellent especially for deep water moorings ranging from $1000-4000 \mathrm{~m}$.

\subsection{Outline of Thesis}

Chapter 2 covers the Jordan Block Perturbation and examines applications to the analytical prediction of moderately localized modes. It also provides convergence zones for the perturbation techniques being used in this thesis.

Chapter 3 offers numerical proof of the fact that modal sensitivity is directly linked to the branch points in the frequency-disorder relation. We investigate the conflicting effects of the order of the branch point and the location in the complex plane.

Chapter 4 outlines the nonlinear optimization methods used for larger systems to determine optimum parameter combinations to ensure some minimum level of localization in the system. Applications of the method and a systematic study of the dependence of the optimum disorder on the minimum localization factor is done. 
We also examine the inverse problem of maximizing localization for some given mean disorder in the system. Studies of the distribution of optima and a statistical analysis to show that the sampling of only a few optima can provide an excellent estimate of the global optimum is also provided. We also use this optimization scheme to search for special configurations which are close to multiple eigenvalue coalescences and satisfy the optimality conditions.

Chapter 5 examines a real world application of mode localization in passive vibration isolation. The structure that is studied is an oceanographic mooring with regularly spaced subsurface buoys. The main source of excitation was the wave induced excitation and the waves were inline elastic waves. The need to reduce vibrations arose because of the presence of instrumentation on the mooring which needed minimum motion for accuracy of measurement. Localization was induced by randomizing the positions of the buoys. It was found to be useful for passive vibration isolation for structures which were in deep waters (1000-4000 m).

Chapter 6 covers conclusions and provides recommendations for future research. 


\section{Chapter 2}

\section{Analytical Prediction of Localized Modes using Perturbation Techniques}

\subsection{Introduction}

We use analytical perturbation techniques to study the modes of oscillation of a system of disordered, coupled pendula as seen in figure 2-1. Pierre and Dowell[27] presented two perturbation methods, called the Classical Perturbation Method (CPM) and Modified Perturbation Method (MPM). The CPM used a set of identical coupled pendula as the unperturbed state. The CPM made the assumption that the disorder was much smaller than the coupling and used the disorder as the parameter in which the perturbation series was expanded. They were however only able to accurately describe modes which appeared almost periodic or "lightly localized". The second expansion (MPM) was about the uncoupled, disordered state. The MPM expansion was written out with the coupling being used as the small parameter for the perturbation series. The assumption here was that the coupling was much smaller than the disorder. The MPM was successful in describing "heavily localized" modes where the modes have significant amplitude on one pendulum with small non-zero amplitude 


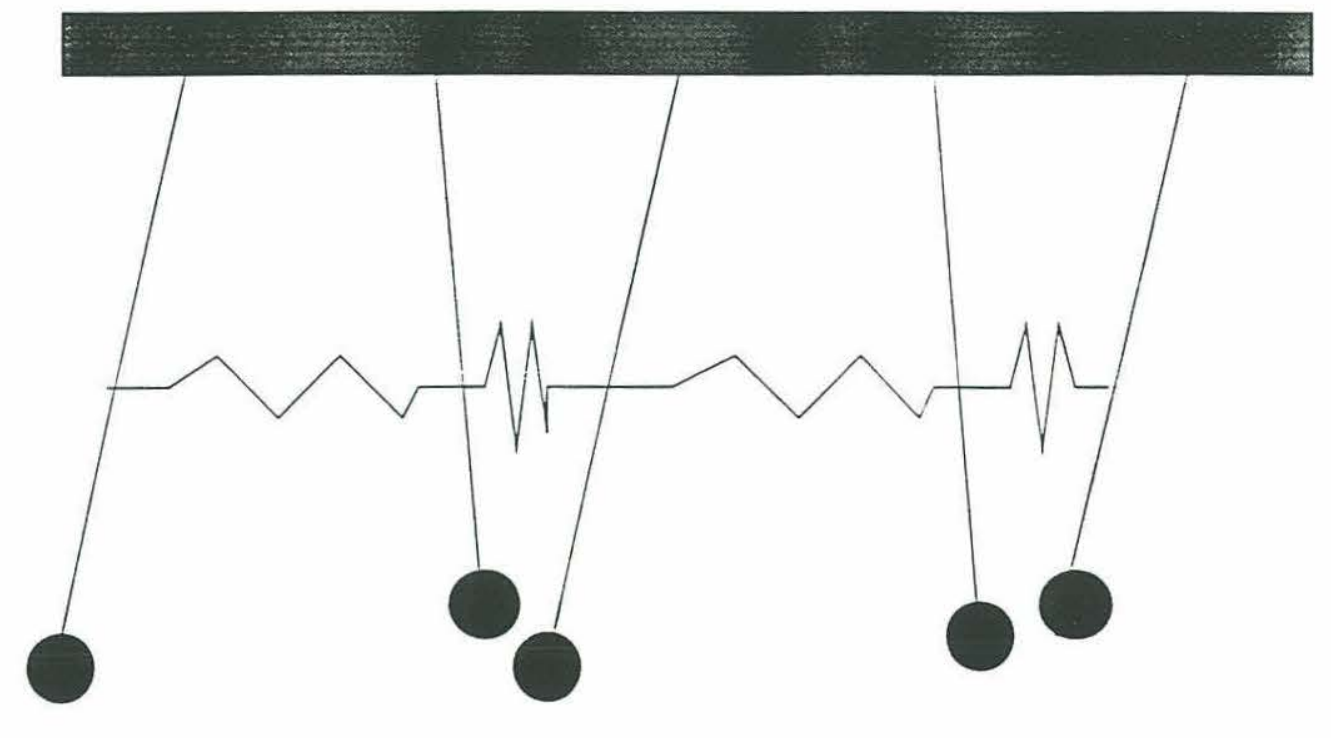

Disordered system of $\mathbf{n}$ coupled pendula

Figure 2-1: A System of Coupled Pendula 
on the other pendula.

Neither of the perturbation expansions work for modes where the coupling is of the same order as the disorder. Triantafyllou and Triantafyllou [39] showed that the CPM and MPM are limited in their zone of convergence because of the existence of branch points in the eigenvalue-disorder relation. We have noted in Chapter 1 that the localization seen in modes varies nonlinearly with the disorder. As disorder is introduced into the system of pendula, the modes abruptly change from periodic to localized passing through the state of moderate localization. Once localized, they show very little change of modes with disorder. The MPM works well over this large zone of disorder over which there is almost no change in the modes.

Moderately localized modes are associated with the intermediate range of parameters where there is large sensitivity of modes in their transition from extended to localized state. In this chapter, we introduce a new perturbation method to describe modes in this intermediate state of localization. The perturbation expansion is performed about branch points in the eigenvalue-disorder relation. It fills in the gap left by the CPM and MPM and allows us to obtain an analytical description of modes in various states of moderate localization.

It must be emphasized that the numerical methods for evaluation of eigenvalues and eigenvectors of matrices are sufficiently evolved to make redundant the usage of perturbation techniques for numerical calculations (especially for the size of matrices we consider for structural dynamics applications which range from two to twenty elements). However, while numerical calculations are important, we require analytical perturbation techniques to provide more physical insights into the problem, such as which parameters affect localization more, what are the parameters influencing the large modal sensitivity and what range of parameters are we more likely to see localization. 



\section{Disordered two pendulum system}

Figure 2-2: System of Two Pendula 


\subsection{Two pendula problem}

We will use the simple case of two pendula as shown in figure 2-2 to demonstrate the differences between the various perturbation expansions. The nominal pendula are of length $l$ and have mass $m$ which are taken equal to unity. The pendula are coupled by a spring with constant $k$. The variation in length of one of the pendula from the nominal length is denoted by $\Delta l$. If we define a nondimensional spring constant $R^{2}=\frac{k l}{m g}$, and disorder $\epsilon=\frac{\Delta l}{l}$, the eigenvalues are

$$
\lambda=R^{2}+\frac{1}{2}+\frac{1}{2(1+\epsilon)} \pm \frac{\sqrt{4 R^{4}+\frac{\epsilon^{2}}{(1+\epsilon)^{2}}}}{2}
$$

The first perturbation expansion that Pierre and Dowell ([27]) advocated was the CPM. Since small perturbations about the disordered state result in dramatic changes to mode shapes, Pierre and Dowell suggested that expansions be performed about the state of zero disorder with the disorder being used as the small parameter for the perturbation expansion. The CPM uses the periodic state as the unperturbed state corresponding to $\epsilon=0$. The two unperturbed eigenvalues are $\lambda=1,1+2 R^{2}$.

The CPM expansion for the eigenvalues as a series in the small parameter $\epsilon$ (where $\epsilon<<R^{2}$ ) would be

$$
\lambda_{1,2}=1+R^{2}-\frac{\epsilon}{2}+\frac{\epsilon^{2}}{2} \pm R^{2}\left(1+\frac{\epsilon^{2}}{8 R^{4}}\right)+\ldots
$$

During the expansion, Pierre and Dowell [27] made the assumption that the parameter $\epsilon$ was small in relation to $R^{2}$ and this assumption is violated as $\epsilon$ becomes larger. Pierre and Dowell found (as we will confirm later in this chapter) that there was very little change in the modes in the range considered and they appeared almost periodic in appearance. He concluded that localization of modes would be seen more in the parameter range where the coupling was much smaller than the disorder $\left(R^{2}<<\epsilon\right)$. Obviously in this range of parameters, the assumption that $\epsilon<<R^{2}$ was violated.

They put forward the MPM as a perturbation method to be used to describe 
modes which were heavily localized. The MPM uses the state where $R^{2}=0$ and $|\epsilon|>0$. The unperturbed eigenvalues are $\lambda=1$ and $\lambda=1-\epsilon$. The MPM expansion for the eigenvalues about the disorder $\epsilon$ as a series in the small parameter $R^{2}$ would be

$$
\lambda_{1,2}=1-\frac{\epsilon}{2} \pm \frac{\epsilon}{2}+R^{2}+\frac{\epsilon^{2}}{2} \mp \frac{\epsilon^{2}}{2} \mp \frac{R^{4}(1+\epsilon)^{2}}{\epsilon^{2}}
$$

This works well for heavily localized modes. However the second order perturbation fails as $\epsilon \rightarrow 0$ because the assumption that $R^{2}$ is very small compared to $\epsilon$ is violated. A very interesting feature of this breakdown is that the method does not break down for small $\epsilon$ if the expansion is terminated at linear order but it breaks down if the expansion is terminated at quadratic order. This breakdown is related to the asymptotic nature of the MPM expansion. When asymptotic series break down, additional terms do not improve the predictive capabilities of the asymptotic series but actually reduce the quality of the prediction and in this case the MPM displays precisely this form of behavior.

However, a gap in the accurate prediction of localized modes still existed. There existed an inability to describe modes in a state of intermediate localization which also corresponded to parameter values where $\epsilon \simeq R^{2}$. This manifested itself mathematically by the presence of branch points in the eigenvalue-disorder relation whose existence we next show. By analytic continuation, we permit the disorder parameter to become complex. The complex length has no physical significance and is mainly an outcome of the application of complex variable theory. Branch points occur in the frequency-disorder relation if

$$
\epsilon_{0}= \pm \frac{2 R^{2} i}{1 \mp 2 R^{2} i}
$$

This is obtained by setting the expression under the square-root in equation 2-1 equal to zero and solving for the disorder $\epsilon$. The new perturbation expansion which we introduce in this chapter is written about the branch point $\epsilon_{0}$. At this point, the eigenvalues are equal and given by 


$$
\lambda_{0}=1+R^{2}-i R^{2}
$$

An expansion about the branch point can be obtained by setting

$$
\zeta=\epsilon-\epsilon_{0}
$$

We can expand the solution to eq. $2-1$ in a series in the complex variable $\zeta^{\frac{1}{2}}$. We get

$$
\lambda_{1,2}=1+R^{2}-i R^{2} \mp \zeta^{\frac{1}{2}} \sqrt{i R^{2}}\left(1-2 i R^{2}\right)-\zeta \frac{\left(1-2 i R^{2}\right)^{2}}{2} \pm \zeta^{\frac{3}{2}} \frac{1}{8 \sqrt{i R^{2}}\left(1-2 i R^{2}\right)}+\ldots
$$

There is an obvious difference in the expansions seen for the MPM and CPM as opposed to expansion about the branch point. There are additional fractional powers of the the small parameter appearing in the expansion. The square-root behavior exhibited by the eigenvalue is exhibited by the eigenvectors also. The eigenvectors of the two pendulum system can be expanded about the branch point to lowest order as

$$
\{q\}=\left\{\begin{array}{c}
1 \\
\frac{-i}{1-2 R^{2} i}
\end{array}\right\} \pm \zeta^{\frac{1}{2}}\left\{\begin{array}{c}
0 \\
\sqrt{i R^{2}}
\end{array}\right\}+\ldots
$$

At the branch point, we have only one distinct eigenvalue and eigenvector for this matrix. The matrix is said to be associated with a Jordan block of size two and the matrix perturbation expansion about the branch point will henceforth be referred to as a Jordan Block perturbation. These branch points occur in complex conjugate pairs. Matrix perturbation techniques have their radii of convergence bounded by the distance to the closest singularity, in this case, the branch point. The CPM and MPM are restricted in their radius of convergence due to the branch point (Triantafyllou and Triantafyllou [39]). The Jordan Block expansion too is restricted in its convergence by the branch points however its convergence zone spans precisely those parameter values where the MPM and CPM breakdown which also corresponds to moderate 

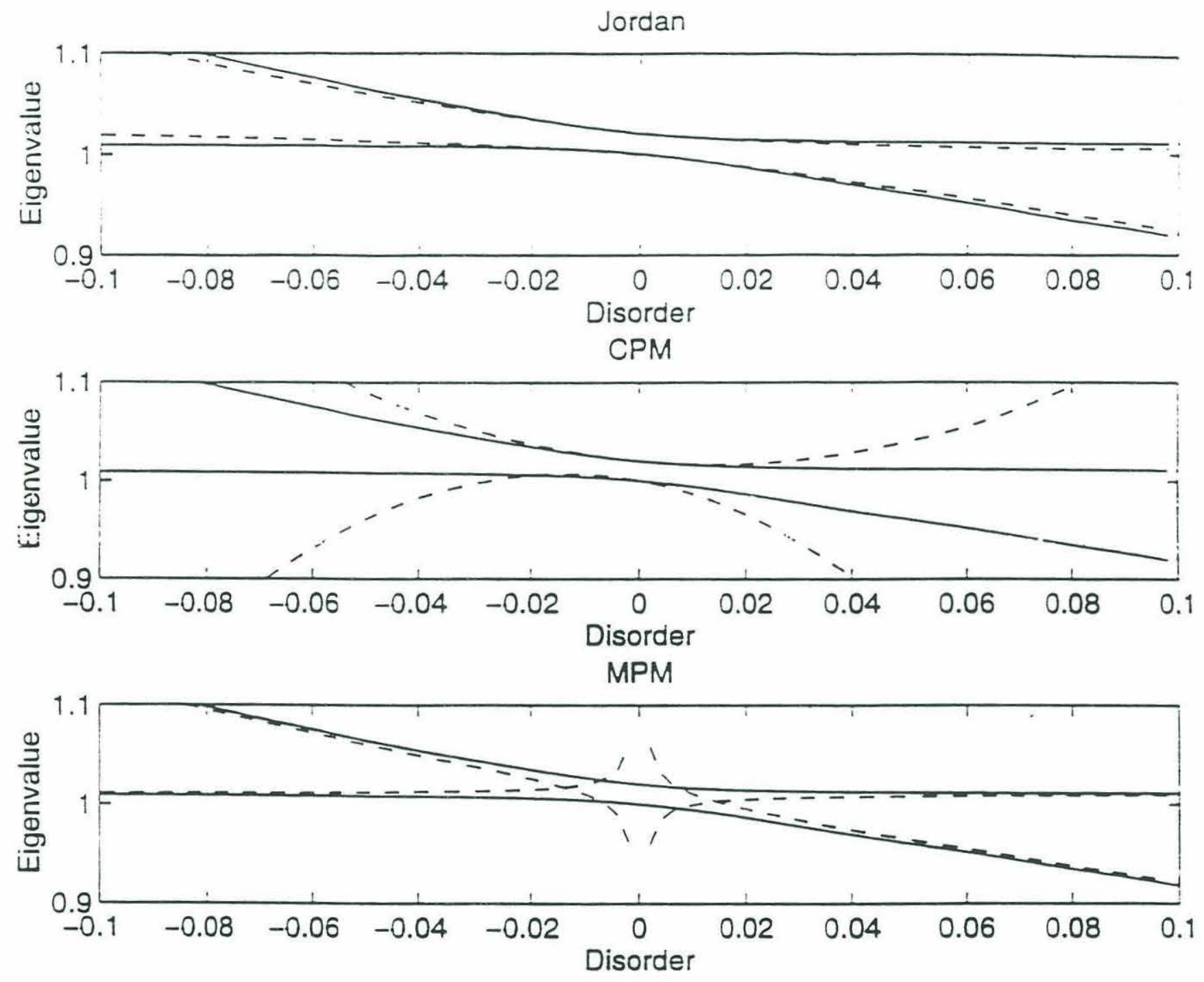

Figure 2-3: Comparison of convergences of Jordan, MPM and CPM Methods. - : Exact. --: Perturbation prediction. 
localization. We can see this by comparing the predictions of the eigenvalues for the three perturbation methods as a function of disorder (Fig 2-3). We consider the range $-.08<\epsilon<.08$. The Jordan block prediction for the eigenvalues is seen to perform well in a significant portion of the range of parameters we consider i.e. $-.04<\epsilon<.04$, while the CPM is seen to work well in the range $-.02<\epsilon<.02$. The MPM works well in the range $\epsilon>.04$ and $\epsilon<-.04$. We will later see that the eigenvector predictions are far poorer than those for the eigenvalues. However this range $.02<|\epsilon|<.04$ where the CPM and MP Mperform poorly, is in fact the range where the Jordan block expansion outperforms the CPM and MPM. We will also show in this chapter that this zone is also a zone of maximum change of the eigenvectors.

Expansion about a branch point would imply that we cannot utilize a standard Taylor series like we saw for the CPM and MPM. We have to use what is called a Pusieux series where rather than having the eigenvalues and eigenvectors vary as integer powers of the disorder, we have the eigenvalues and eigenvectors vary as fractional powers of the disorder parameter (Gohberg et al. [14]). In matrix perturbation theory, a Pusieux series is associated with perturbations about a Jordan block. In

general for an $n$th root branch point, we could have an expansion in the $n$th root of the complex parameter $\zeta$ and an association with a Jordan block of size $n$. The Jordan Block of size $n$ would only have one distinct eigenvector and $n$ repeating eigenvalues.

\subsection{Procedure for $n$-order Jordan block expan- sion}

The general system of $n$ pendula has the following stiffness matrix

$$
[K]=\operatorname{Tridiag}\left[-R^{2} \frac{\left(1+\epsilon_{j-2}\right)}{\left(1+\epsilon_{j-1}\right)} ; \frac{1}{\left(1+\epsilon_{j-1}\right)}+\left(2-\delta_{j, n}-\delta_{j, 1}\right) R^{2} ;-R^{2} \frac{\left(1+\epsilon_{j}\right)}{\left(1+\epsilon_{j-1}\right)}\right]
$$

where $1 \leq j \leq n$. The notation Tridiag $\left(\alpha_{j}, \beta_{j}, \kappa_{j}\right)$ designates a tridiagonal matrix with $\alpha_{j}$ being the element of the lower diagonal ( $j$ th row, $(j-1)$ th column), $\beta_{j}$ is the element on the main diagonal (jth row, jth column), and $\kappa_{j}$ is the element on the 
upper diagonal ( $j$ th row, $(j+1)$ th column). By definition $\alpha_{1}=\kappa_{n}=0$. Also $\delta_{i, j}$ is the Kronecker Delta function and is defined as

$$
\delta_{i, j}= \begin{cases}1 & \text { if } \mathrm{i}=\mathrm{j} \\ 0 & \text { otherwise }\end{cases}
$$

The variables in the above equation are

$\epsilon_{j}$ : Disorder of $j$ th pendulum. The pendula are numbered from left to right in figure

$2-1$ with $0 \leq j \leq(n-1)$.

$R^{2}=\frac{k l}{m g}$ : Nondimensional coupling parameter

k: Coupling spring stiffness.

g: Acceleration due to gravity.

m: Mass of pendulum.

l: Length.

We write out the perturbation expansion about the branch point in the complex disorder space.

$$
\left[K_{0}+\delta K+\delta^{2} K+\ldots\right]\left\{x_{01}+\delta^{\frac{1}{n}} x+\ldots\right\}=\left(\lambda_{0}+\delta^{\frac{1}{n}} \lambda+\ldots\right)\left\{x_{01}+\delta^{\frac{1}{n}} x+\ldots\right\}
$$

The stiffness matrix at the complex branch is $K_{0}$. We write out the ordered problem

$O(1)$

$$
\left[K_{0}-\lambda_{0} I\right] x_{01}=0
$$

$O\left(\epsilon^{\frac{1}{n}}\right)$

$$
\left[K_{0}-\lambda_{0} I\right]\left\{\delta^{\frac{1}{n}} x\right\}=\delta^{\frac{1}{n}} \lambda\{x\}
$$

$O\left(\epsilon^{\frac{2}{n}}\right)$

$$
\left[K_{0}-\lambda_{0} I\right]\left\{\delta^{\frac{2}{n}} x\right\}=-\left[\delta K-\lambda_{1} I\right]\left\{x_{01}\right\}+\left(\delta^{\frac{1}{n}} \lambda\right) \delta^{\frac{1}{n}} x
$$




$$
\begin{aligned}
& O\left(\epsilon^{2+\frac{n-1}{n}}\right) \\
& {\left[K_{0}-\lambda_{0} I\right]\left\{\left\{\delta^{2+\frac{n-1}{n}}\{x\}\right\}=-[\delta K-\delta \lambda]\left[\delta^{1+\frac{n-1}{n}} x\right]-\left[\delta^{2} K-\delta^{2} \lambda I\right]\left[\delta^{\frac{n-1}{n}} x\right]+\right.} \\
& \left(\delta^{\frac{1}{n}} \lambda\right)\left[\delta^{2+\frac{n-2}{n}} x\right]+\ldots+\left(\delta^{2+\frac{n-1}{n}} \lambda\right) x_{01}
\end{aligned}
$$

All the quantities in the superscripts attached to $\delta$ indicate the order of magnitude associated with those quantities. Thus $\delta^{\frac{1}{n}} \lambda$ indicates an $n$th root perturbation in $\lambda$ and $\left[\delta^{\frac{1}{n}} x\right]$ represents the $n$th root perturbation in the eigenvectors and so on. These equations govern the perturbations to the eigenvalue and eigenvectors at each order of the perturbation. We write out the expansions to order $\epsilon^{2+\frac{(n-1)}{n}}$ because (as we will show later) in order that we solve the complete perturbation to order $O\left(\epsilon^{2}\right)$ we have to utilize the perturbation equations to order $\epsilon^{2+\frac{(n-1)}{n}}$.

The first order and second order perturbed stiffness matrices about the periodic state are given by

$$
\delta K=\operatorname{Tridiag}\left[-R^{2}\left(\zeta_{j-2}-\zeta_{j-1}\right) ;-\zeta_{j-1} ;-R^{2}\left(\zeta_{j}-\zeta_{j-1}\right)\right]
$$

and

$$
\delta^{2} K=\operatorname{Tridiag}\left[-R^{2}\left(\zeta_{j-1}^{2}-\zeta_{j} \zeta_{j-1}\right) ; \zeta_{j-1}^{2} ;-R^{2}\left(\zeta_{j-1}^{2}-\zeta_{j} \zeta_{j-1}\right)\right]
$$

The first step in the method is to determine the complex coordinates associated with the branch point, and the eigenvalues and eigenvectors associated with the unperturbed state. This unperturbed state is the zero-order problem. The eigenvector associated with the Jordan block obeys the standard eigenvector relation at the branch point, 


$$
\left[K_{0}-\lambda_{0} I\right] x_{01}=0
$$

The Jordan Block perturbation method used the branch points in the modedisorder relation as the unperturbed state. This is determined easily for a system of two coupled pendula. However for larger systems, we require mathematical equations to determine the complex disorder parameters which define the branch point. The characteristic polynomial of the eigenvalue problem is given by

$$
\Delta\left(\lambda, \epsilon_{1}, \ldots \epsilon_{n-1}\right)=|K-\lambda I|=0
$$

where the vertical bar denotes the determinant. The mathematical conditions for eigenvalue coalescence to occur can be derived by considering the form of the characteristic polynomial at the point of eigenvalue coalescence(Triantafyllou and Triantafyllou [39]). The form of the polynomial at the point of $m$ root coalescence would be

$$
\Delta \simeq\left(\lambda-\lambda_{0}\right)^{m}
$$

where $\lambda_{0}$ is the coalescent eigenvalue. The condition for the coalescence of $m$ eigenvalues would be

$$
\frac{\partial^{i} \Delta}{\partial \lambda^{i}}=0
$$

and $i=1, \ldots, m-1$ with $m \leq n$. This is in fact a condition for a saddle point to occur. Along with the equation for the characteristic polynomial, we have a system of $m$ equations. We require $m$ unknown variables to be guaranteed a solution to this system of $m$ equations.

If $m=n$ which would then correspond to an $n$th root branch point, we would have to solve for the complex unknowns $\left(\lambda, \epsilon_{1}, \ldots \epsilon_{n-1}\right)$. The $n$ root coalescence is a "fixed" singularity. We get a set of isolated discrete points as the solution to the equations for eigenvalue coalescence. According to Bender and Orzsag [3], for a problem where 
the dependence on the disorder parameter is linear, the number of $n$ root coalescences would be $n$ ! if the characteristic polynomial is of order $n$.

Having determined the complex coordinates of $n$ root coalescence, the next step is to calculate the eigenvectors associated with the Jordan block. We can only determine one eigenvector associated with the Jordan Block since the Jordan block of size greater than one is associated with a matrix of reduced rank. We however need a set of $n$ eigenvectors to span the $n$ dimensional space. This is done by constructing a special set of vectors called generalized eigenvectors which along with the single eigenvector span the $n$ dimensional space. The generalized eigenvectors satisfy the following relation (Gohberg et. al. [14] and Wilkinson [41]),

$$
\left[K_{0}-\lambda_{0} I\right] x_{0 i}=x_{0(i-1)}
$$

where, $2 \leq i \leq n$.

These eigenvectors of the unperturbed state are the set of basis functions we use for expanding the eigenvector perturbations at each order of the perturbation. The eigenvectors at each order of the perturbation are expanded as linear combinations of the unperturbed eigenvectors

$$
\delta^{\frac{m}{n}} x=\sum_{j=1}^{j=n} c_{\frac{m}{n}, j} x_{0 j}
$$

Note $m$ denotes the perturbation order and $m=1,2, \ldots$. During the perturbation expansion, we have $(n+1)$ unknowns. These are the unknown eigenvalue perturbation(one unknown) and the $n$ coefficients ( $n$ unknowns $c \frac{m}{n}, j$ ) which are used to linearly combine the $n$ eigenvectors when we compute the eigenvector perturbation. However, we can only generate $n$ equations by systematically multiplying the perturbation equation by the $n$ left eigenvectors. We need one more equation to ensure that we have $n+1$ equations to solve for $n+1$ unknowns. This is obtained as follows. The eigenvectors which are perturbed must satisfy the orthogonality conditions between the right and left eigenvectors at all orders of the perturbation. The left generalized eigenvector $y_{01}^{H}$ is the reciprocal of the right eigenvector. As we perturb the vector 
away from the Jordan Block, we get $n$ splits in the solution. A split implies that as we perturb the solution away from the Jordan block vectors, we get $n$ solutions emerging from a single vector corresponding to the Jordan block. Thus,

$$
y_{01}^{H}\left(x_{01}+\delta^{\frac{1}{n}} x+\delta^{\frac{2}{n}} x+\ldots\right)=1
$$

Ordering terms, at all orders, we get

$$
y_{01}^{H} x_{01}=1
$$

and

$$
y_{01}^{H} \delta^{\frac{m}{n}} x=0
$$

During the perturbation expansion, we require the left eigenvectors as a set of orthogonal vectors to determine the coefficients multiplying the generalized right eigenvectors. Hence we next calculate the left eigenvectors.

Although at the branch point, we have one left and one right eigenvector, they are orthogonal to each other. The reciprocal of each eigenvector is a generalized eigenvector.

At the branch point, we only have one left eigenvector which obeys the following relation

$$
y_{0 n}^{H}\left[K_{0}-\lambda_{0} I\right]=0
$$

where the superscript $H$ denotes the hermitian operation of transpose and conjugate. The left generalized vectors satisfy the following relations.

$$
y_{0 i}^{H}\left[K_{0}-\lambda_{0} I\right]=y_{0(i+1)}^{H}
$$

with $1 \leq i \leq n-1$. The left and right eigenvectors if chosen correctly, will obey orthogonality relations 


$$
y_{0 i}^{H} x_{0 j}=\beta \delta_{i, j}
$$

Here, 3 is a normalizing constant which is taken as one and $\delta_{i, j}$ is the Kronecker delta function.

We briefly outline the solution procedure. The perturbation problem to order $\frac{m}{n}$ is said to be solved if we obtain solutions for all of the $(n+1)$ unknowns $\delta^{\frac{m}{n}} \lambda, c \frac{m}{n}, 1, \ldots, c \frac{m}{n}, n$. In perturbation methods for matrices with distinct eigenvalues, we are able to obtain the entire solution to order $m$ by solely utilizing the equations from that order of the perturbation. This is not true for the Jordan block expansion.

$c_{\frac{m}{n}, 1}$ can easily be determined using the orthogonality condition applied to the perturbed eigenvector(equation 2-24). We introduce the expansion in equation (2-23) into equation (2-24) to get

$$
c_{\frac{m}{n}, 1}=0
$$

Thus the eigenvector associated with the Jordan Block is only perturbed in the direction of the generalized eigenvectors.

We still have $n$ unknowns to determine. We use the perturbation equations at different orders to determine these coefficients. Each of these equations are matrix equations. Each of them are reduced to $n$ scalar equations by multiplying successively by the left eigenvectors $y_{0 j}, j=1, \ldots, n$.

At the order $m$, we can obtain only two useful equations, the first is the orthogonality condition for the perturbed eigenvector at that order and the second equation is that obtained by multiplying the order $m$ perturbation with $y_{01}^{H}$. We obtain the remaining $(n-1)$ equations by multiplying each of the successive $(n-1)$ order equations by the eigenvectors $y_{02}^{H}, y_{03}^{H}, \ldots$ and,$y_{0 n}^{H}$ respectively. These equations are constructed so that even though they are obtained from utilizing perturbations equation whose order is greater than $m$, they still couple the unknowns of the order $m$ problem only. Thus, in order to solve for the unknowns at order $m$. we have to utilize equations to order $\frac{m}{n}+\frac{n-1}{n}$. 
The solutions for the two lowest orders are as follow:

$O\left(\epsilon^{\frac{1}{n}}\right)$

$$
\delta^{\frac{1}{n}} \lambda=e^{\frac{2 \pi j i}{n}}\left(\frac{y_{0 n}^{H}(\delta K) x_{01}}{y_{01}^{H} x_{01}}\right)^{\frac{1}{n}}
$$

Note the $n$th roots of unity imply the presence of $\mathrm{n}$ splits in the solution and $i=\sqrt{-1}$ in equation $2-31$.

$$
\begin{gathered}
c_{\frac{1}{n}, 2}=\left(\delta^{\frac{1}{n}} \lambda\right) \frac{\left(y_{01}^{H} x_{01}\right)}{\left(y_{02}^{H} x_{02}\right)} \\
c_{\frac{1}{n}, 3}=\ldots=c_{\frac{1}{n}, n}=0
\end{gathered}
$$

We note that most of the coefficients multiplying the various generalized eigenvectors are zero until we reach integral or higher powers of the perturbation. The $n$th root dependence of the eigenvalue on disorder is transmitted to the eigenvectors also since the eigenvector coefficients depend on the eigenvalue perturbation.

$$
O\left(\epsilon^{\frac{2}{n}}\right)
$$

The trivial solutions to the $O\left(\epsilon^{\frac{2}{n}}\right)$ problem are written below

$$
c_{\frac{2}{n}, 4}=\ldots=c_{\frac{2}{n}, n}=0
$$

There is no simple closed form solution to the remaining non-trivial unknowns. They are obtained as solutions to the system of simultaneous, linear equations obtained by multiplying equations of $O\left(\epsilon^{\frac{2}{n}}\right)$ through $O\left(\epsilon^{\frac{2}{n}+\frac{n-1}{n}}\right)$ by the generalized eigenvectors $y_{01}^{H}, y_{02}^{H}, \ldots, y_{0(n-1)}^{H}, y_{0 n}^{H}$ successively. The equations which were generated are written below.

$$
c_{\frac{2}{n}, 2}\left(y_{02}^{H} x_{02}\right)-\delta^{\frac{2}{n}} \lambda\left(y_{01}^{H} x_{01}\right)=0
$$




$$
\delta^{\frac{1}{n}} \lambda c_{1, n}\left(y_{0 n}^{H} x_{0 n}\right)+\delta^{\frac{2}{n}} \lambda c_{1-\frac{1}{n}, n}\left(y_{0 n}^{H} x_{0 n}\right)-y_{0 n}^{H} \delta K x_{02} c_{\frac{1}{n}, 2}=0
$$

Higher order terms can be systematically obtained in this manner.

We have so far consider cases where a stiffness matrix of size $n * n$ is associated with a Jordan block of size $n$. We could also have situations where the Jordan block of size is of size $m$ where $m<n$. Thus we can have $m$ coincident eigenvalues and $m$ root branch point between the eigenvalue and disorder. The remaining $(n-m)$ eigenvalues are distinct and are associated with $(n-m)$ distinct eigenvectors. At this $m$ th order branch point we have one Jordan block of size $m$ and $(n-m)$ blocks of size one. We apply a hybrid of the Jordan block expansion and the expansion for matrices wit distinct eigenvalues.

The unperturbed state is slightly different from the $m=n$ case. Consider the case where $m<n$ in equation 2-21. Along with the coalescent frequency, we can select $m-1$ parameters, say $\epsilon_{1}, \ldots, \epsilon_{m-1}$ to be unknowns. This would imply that we have to provide arbitrary values to the remaining $(n-m+1)$ parameters $\epsilon_{m}, \ldots, \epsilon_{n-1}$. In this sense, the lower order coalescences are "movable" singularities. Depending on the value we fix for $\epsilon_{m}, \ldots, \epsilon_{n-1}$, we can get different values for the complex branch point coordinates. Thus rather than having a branch point we would have a branch point surface by allowing these arbitrarily fixed parameters to vary in a continuous fashion over the entire field of complex numbers. Next, we should realize that we could have taken another set of disorder parameter say $\epsilon_{n-m}, \ldots, \epsilon_{n-1}$ as our unknowns. We could in fact select $m-1$ of these parameters in $\frac{n !}{(m-1) !(n-m+1) !}$ ways. Hence we have an infinite number of points about which we could perform an $m$ th root expansion where $m<n$. For an $m$ root coalescence, with linear dependence of the characteristic polynomial on disorder (Bender and Orzsag [3]), we could have a maximum of $\frac{n !}{(n-m) !}$ possible $m$ root coalescences given a fixed set of values for the $(n-m+1)$ complex parameters. Since we could select these parameters in $\frac{n !}{(m-1) !(n-m+1) !}$ ways, we would have a total of $\frac{(n !)^{2}}{(n-m) !(m-1) !(n-m+1) !} m$ th root branch point surfaces for an $m$ th root branch point. 


\subsubsection{Application of Jordan Block Expansion to Two Pen- dula}

We next work out the perturbation expansion for a two pendula problem as an example. The perturbation expansion is as follows :

$\left[K_{0}+\delta K+\delta^{2} K+\ldots\right]\left\{x_{01}+\delta^{\frac{1}{2}} x+\delta x+\ldots\right\}=\left(\lambda_{0}+\delta^{\frac{1}{2}} \lambda+\delta \lambda+\ldots\right)\left\{x_{01}+\delta^{\frac{1}{2}} x+\delta x+\ldots\right\}$

The first order perturbed stiffness matrix about the branch point is given by

$$
\delta K=\left(\begin{array}{cc}
0 & -R^{2} \zeta \\
R^{2} \frac{\zeta}{\left(1+\epsilon_{0}\right)^{2}} & -R^{2} \frac{\zeta}{\left(1+\epsilon_{0}\right)^{2}}
\end{array}\right)
$$

and the second order perturbed stiffness matrix is

$$
\delta^{2} K=\left(\begin{array}{cc}
0 & 0 \\
-\frac{R^{2} \zeta^{2}}{\left(1+\epsilon_{0}\right)^{3}} & \frac{R^{2} \zeta^{2}}{\left(1+\epsilon_{0}\right)^{3}}
\end{array}\right)
$$

Note, the subscripts indicate the order of magnitude of the associated quantities. Thus all quantities with subscript $\frac{1}{2}$ are associated with $\epsilon^{\frac{1}{2}}$ and so on. The appearance of fractional powers in the expansion is a direct outcome of the properties of the Jordan block. We write out the ordered problem :

$O(1)$

$$
\left[K_{0}-\lambda_{0} I\right] x_{01}=0
$$

$$
O\left(\epsilon^{\frac{1}{2}}\right)
$$

$$
\left[K_{0}-\lambda_{0} I\right]\left\{\delta^{\frac{1}{2}} x\right\}=\delta^{\frac{1}{2}} \lambda\{x\}
$$

$O(\epsilon)$

$$
\left[K_{0}-\lambda_{0} I\right]\{\delta x\}=-[\delta K-\delta \lambda I]\{x\}+\left(\delta^{\frac{1}{2}} \lambda\right) \delta^{\frac{1}{2}} x
$$




$$
\begin{gathered}
O\left(\epsilon^{\frac{3}{2}}\right) \\
{\left[K_{0}-\lambda_{0} I\right]\left\{\delta^{\frac{3}{2}}\right\} x=-\left[\delta K-\delta^{1} \lambda I\right]\left\{\delta^{\frac{1}{2}} x\right\}+\left(\delta^{\frac{1}{2}} \lambda\right)\{\delta x\}+\left(\delta^{\frac{3}{2}} \lambda\right)\{x\}} \\
O\left(\epsilon^{2}\right) \\
{\left[K_{0}-\lambda_{0} I\right]\left\{\delta^{2} x\right\}=-\left[\delta^{2} K-\delta^{2} \lambda\right]\{x\}-[\delta K-(\delta \lambda) I]\{\delta x\}+(\delta \lambda)(\delta x)+\left(\delta^{\frac{1}{2}} \lambda\right)\left(\delta^{\frac{3}{2}} x\right)+\left(\delta^{\frac{3}{2}} \lambda\right)\left(\delta^{\frac{1}{2}} x\right)} \\
O\left(\epsilon^{\frac{5}{2}}\right) \\
{\left[K_{0}-\lambda_{0} I\right]\left\{\delta^{\frac{5}{2}} x\right\}=-\left[\delta^{2} K-\delta^{2} \lambda I\right]\left\{\delta^{\frac{1}{2}} x\right\}-[\delta K-\delta \lambda I]\left\{\delta^{\frac{3}{2}} x\right\}+\left(\delta^{\frac{1}{2}} \lambda\right)\left(\delta^{2} x\right)+\left(\delta^{\frac{3}{2}} \lambda\right)\left(\delta^{\frac{1}{2}} x\right)}
\end{gathered}
$$

Unlike the CPM and MPM, we have complex perturbation matrices. The right and left eigenvectors and generalized eigenvectors for the unperturbed state are as follow:

$$
\begin{gathered}
{[X]=\left[\begin{array}{ll}
x_{01} & x_{02}
\end{array}\right]=\left[\begin{array}{cc}
1 & 0 \\
-\frac{i}{\left(1-2 i R^{2}\right)} & -\frac{1}{\left(1-2 i R^{2}\right)}
\end{array}\right]} \\
{[Y]^{H}=\left[\begin{array}{ll}
y_{01}^{H} & y_{02}^{H}
\end{array}\right]=\left[\begin{array}{cc}
-\frac{1}{R^{2}\left(1-2 i R^{2}\right)} & 0 \\
\frac{i}{\left(1-2 i R^{2}\right)} & 1
\end{array}\right]}
\end{gathered}
$$

If we apply the orthogonality conditions, we get

$$
[Y]^{H}[X]=[I]
$$

and

$$
Y^{H}[K][X]=\left[\begin{array}{cc}
\lambda_{0} & 1 \\
0 & \lambda_{0}
\end{array}\right]
$$

where the coalescence frequency is $\lambda_{0}=1+R^{2}-i R^{2}$.

We shall solve the complete perturbation problem to $O\left(\epsilon^{2}\right)$ only. As in classical matrix perturbation, we will expand the eigenvector perturbation at each order as a 
linear combination of the basis vectors. If $m$ is the order of the perturbation,

$$
\delta^{\frac{m}{2}} x=c_{\frac{m}{2}, 1} x_{01}+c_{\frac{m}{2}, 2} x_{02}
$$

The problem is said to be completely solved to any order $m$ if we have solved for $c_{m, 1}, c_{m, 2}$ and $\delta^{m} \lambda$. As we noted in the general $n$ pendula problem, if we wish to solve the unknowns at order $m$, we will only get one useful equation at order $m$. The first equation is that obtained by using the orthogonality condition for the perturbed vector at order $m$. We get

$$
y_{01}^{H}\left[\delta^{\frac{m}{2}} x\right]=0
$$

Applying equation (2-50) to (2-51), we get

$$
c_{\frac{m}{2}, 1}=0
$$

The unknowns $c_{\frac{m}{2}, 2}$ and $\delta^{\frac{1}{2}} \lambda$ are obtained by solving two simultaneous linear equations. The first equation is obtained by multiplying the order $m$ equation by $y_{02}^{H}$. This is the second useful equation at order $m$. As we noted in the general size $n$ Jordan block expansion problem, we can obtain $(n-1)$ (in this case one) more useful equation(s) by using the order $\frac{m}{n}+\frac{1}{n}$ through $\frac{m}{n}+\frac{(n-1)}{n}$ order perturbation equations. The second equation is obtained by multiplying the order $\left(\frac{m}{2}+\frac{1}{2}\right)$ equation by $y_{02}^{H}$.

The solutions to order $O\left(\epsilon^{2}\right)$ is given below.

$$
\begin{aligned}
& c_{\frac{1}{2}, 1}=0 \\
& c_{\frac{1}{2}, 2}=\delta^{\frac{1}{2}} \lambda \\
& \delta^{\frac{1}{2}} \lambda= \pm \sqrt{\frac{y_{02}^{H}(\delta K) x_{01}}{y_{01}^{H} x_{01}}}
\end{aligned}
$$




$$
\begin{aligned}
& c_{1,1}=0 \\
& c_{1,2}=\frac{\frac{y_{02}^{H}(\delta K) x_{01}}{y_{02}^{H} x_{02}}-\frac{y_{02}^{H}(\delta K) x_{01}}{y_{01}^{H} x_{01}}}{1+\frac{y_{02}^{H} x_{02}}{y_{01}^{H} x_{01}}} \\
& \delta \lambda=\frac{y_{01}^{H}(\delta K) x_{02}+y_{01}^{H}(\delta K) x_{02}}{y_{01}^{H} x_{01}+y_{02}^{H} x_{02}} \\
& O\left(\epsilon^{\frac{3}{2}}\right) \\
& c_{\frac{3}{2}, 1}=0 \\
& c_{\frac{3}{2}, 2}=\frac{\frac{y_{02}^{H}\left(\delta^{2} K\right) x_{01}}{y_{01}^{H} x_{01} \delta^{\frac{1}{2}} \lambda}+\frac{\left(y_{02}^{H}(\delta K) x_{02}\right) c_{1,1}}{y_{02}^{H} x_{02} \delta^{\frac{1}{2}} \lambda}-\frac{\delta \lambda c_{1,1}}{\delta^{\frac{1}{2}} \lambda}-\frac{y_{01}^{H}(\delta K) x_{02} \delta^{\frac{1}{2}} \lambda}{y_{01}^{H} x_{01}}}{\left(1+\frac{y_{02}^{H} x_{02}}{y_{01}^{H} x_{01}}\right)} \\
& \delta^{\frac{3}{2}} \lambda=\frac{\frac{y_{02}^{H}\left(\delta^{2} K\right) x_{02}}{\delta^{\frac{1}{2}} \lambda}+\frac{y_{02}^{H}(\delta K) x_{02} c_{1,2}}{\delta^{\frac{1}{2}} \lambda}-\frac{\delta \lambda\left(y_{02}^{H} x_{02}\right) c_{1,2}}{\delta^{\frac{1}{2}}(\lambda)}+\left(\delta^{\frac{1}{2}} \lambda\right) y_{01}^{H}(\delta K) x_{02}}{y_{01}^{H} x_{01}+y_{02}^{H} x_{02}} \\
& O\left(\epsilon^{2}\right) \\
& c_{2,1}=0 \\
& c_{2,2}=\frac{-y_{01}^{H}\left(\delta^{2} K\right) x_{01}-y_{01}^{H}(\delta K) x_{02} c_{1,2}+\frac{\left(y_{01}^{H} x_{01}\right)\left(y_{02}^{H}\left(\delta^{2} K\right) x_{02}\right)}{\left(y_{02}^{H} x_{02}\right)}+\frac{\left(y_{02}^{H}(\delta K) x_{02} c_{\frac{3}{2}, 2}\right)\left(y_{01}^{H} x_{01}\right)}{\left(\delta^{\frac{1}{2}} \lambda\right)\left(y_{02}^{H} x_{02}\right)}}{y_{01}^{H} x_{01}+y_{02}^{H} x_{02}} \\
& \frac{-\frac{\left(\delta^{\frac{3}{2}} \lambda\right) c_{1,2}\left(y_{01}^{H} x_{01}\right)}{\delta^{\frac{1}{2}} \lambda}-\frac{\delta \lambda c_{\frac{3}{2}, 2}\left(y_{01}^{H} x_{01}\right)}{\left(\delta^{\frac{1}{2}} \lambda\right)}}{y_{01}^{H} x_{01}+y_{02}^{H} x_{02}}
\end{aligned}
$$




$$
\begin{gathered}
\delta^{2} \lambda=\frac{y_{02}^{H}\left(\delta^{2} K\right) x_{02}+\frac{y_{02}^{H}(\delta K) x_{02}}{\delta^{\frac{1}{2}} \lambda}-\frac{(\delta \lambda) c_{\frac{3}{2}, 2}\left(y_{02}^{H} x_{02}\right)}{\delta^{\frac{1}{2}} \lambda}-\frac{\delta^{\frac{3}{2}} \lambda\left(y_{02}^{H} x_{02}\right) c_{1,2}}{\left(\delta^{\frac{1}{2}} \lambda\right)}+\left(y_{01}^{H}\left(\delta^{2} K\right) x_{01}\right)}{y_{01}^{H} x_{01}+y_{02}^{H} x_{02}}+ \\
\frac{y_{01}^{H}(\delta K) x_{02} c_{1,2}}{y_{01}^{H} x_{01}+y_{02}^{H} x_{02}}
\end{gathered}
$$

The above represents the solution for the Jordan Block size two perturbation expansion to second order.

We confirm our results with the closed form solution derived earlier in equation (2-7) and (2-8) by applying the order $O\left(\epsilon^{\frac{1}{2}}\right)$ solution.

$$
\begin{aligned}
& \delta^{\frac{1}{2}} \lambda= \pm \zeta^{\frac{1}{2}} \sqrt{i R^{2}}\left(1-2 i R^{2}\right) \\
& c_{\frac{1}{2}, 2}= \pm \zeta^{\frac{1}{2}} \sqrt{i R^{2}}\left(1-2 i R^{2}\right)
\end{aligned}
$$

The predictions of the expansion match the closed form expansion derived earlier(equations (2-7) and (2-8)).

It should be noted that the perturbation technique laid out here for the Jordan form and nondegenerate coalescences is different from that for matrices with degenerate coalescences. There are $n$ eigenvectors associated with the size $n$ matrix unlike the Jordan form where we have fewer eigenvectors than the size of the matrix. The eigenvalues and eigenvectors associated with a degenerate coalescence can be expanded in a Taylor series (Courant and Hilbert [11]) about the point of eigenvalue coalescence.

We next compare the predictions of the three perturbation techniques in figure 2 -4. We retain terms to $O\left(\epsilon^{2}\right)$ in our calculations. We define an error norm as follows

$$
e=\max \left(\left|\gamma_{i}^{a}-\gamma_{i}^{p}\right|\right)
$$

Here $\gamma_{i}^{a}$ is the localization factor of the $i$ th actual eigenvector and $\gamma_{i}^{p}$ is the localization factor of the $i$ th predicted eigenvector. The localization factor definition is provided in Appendix A. The procedure to calculate the localization factor is as 


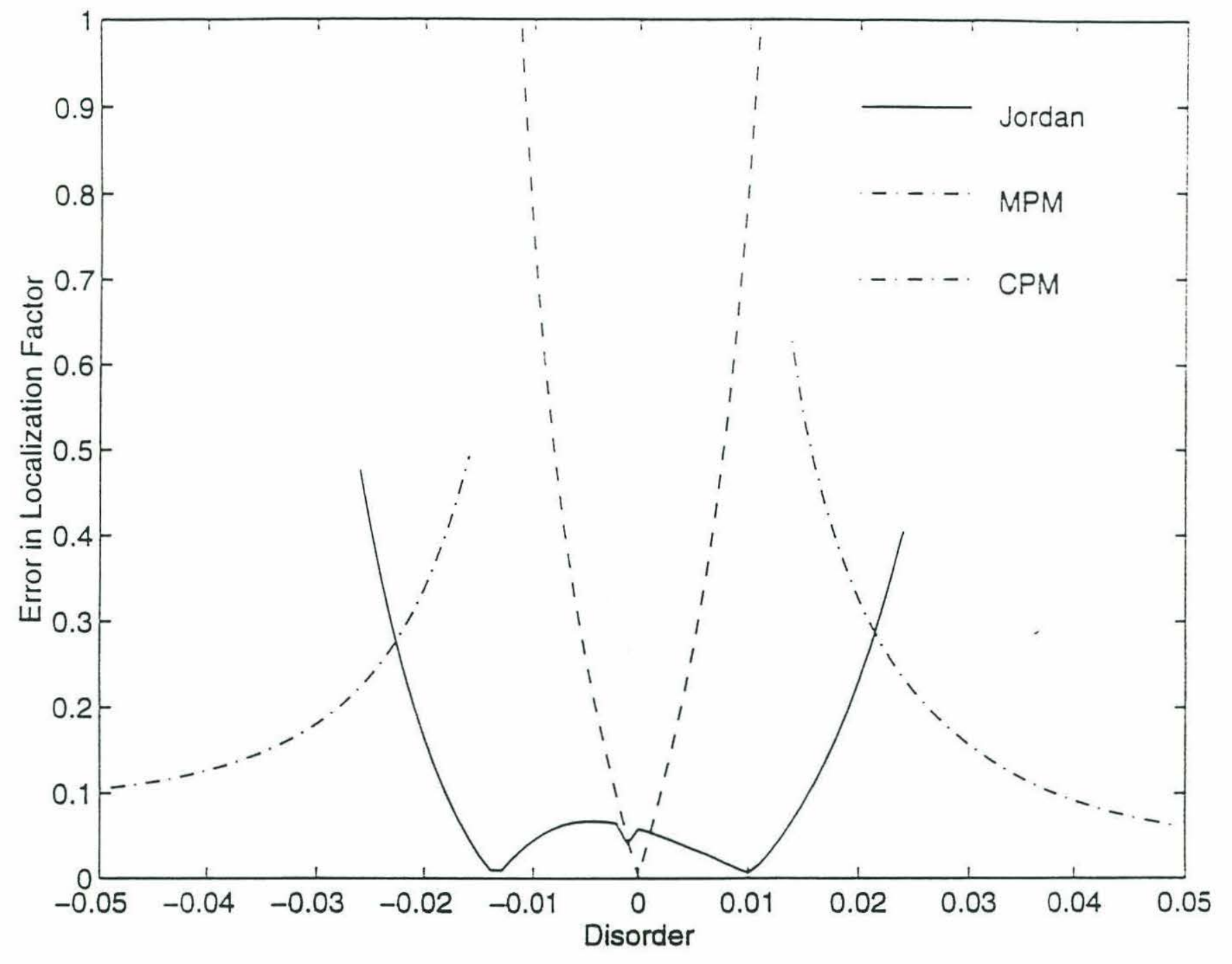


follows :

- Compute the logarithm of the absolute modal coordinate for each mode. Determine the peak.

- If the peak is at the end oscillator, fit a straight line through the logarithm of the modal coordinates. The slope of this line is the localization factor. If the peak is at a middle oscillator, compute the two localization factors for the decay in the mode on either side of the peak. Average the two values to obtain a single localization factor value for the mode.

We use the maximum of these values since the prediction is as good as the poorest prediction. When the actual and predicted eigenvector are close, the error norm is small and the error is a continuous function of the eigenvector. However when they become poor in their fit, we start getting very poor and discontinuous variation of the function with the disorder.

In figure $2-4$, we provide a plot of the variation of the error norm $e$ associated with the eigenvector. The MPM and CPM are less effective than the Jordan Block Expansion in the range of parameters $|\epsilon| \leq .02$ except right at the origin. The MPM appears to be accurate over a large range of parameters $(|\epsilon|>.04)$. But, the modes change very little over that range. The Jordan Block method is valid over a smaller range $(|\epsilon|<.04$. But the modes have maximum sensitivity in that range $(.02<|\epsilon|<.04)$ as they change from a periodic to a localized state.

\subsection{Higher Order Systems}

The two pendula problem was simple in that we had only one branch point (and its complex conjugate) to perform perturbation expansions about. There is increasing complexity in higher order branch points owing to the presence of branch points of different orders. We examine a system of three pendula (figure 2-5) to illustrate these ideas. The eigenvalue problem associated with the three pendula system is 


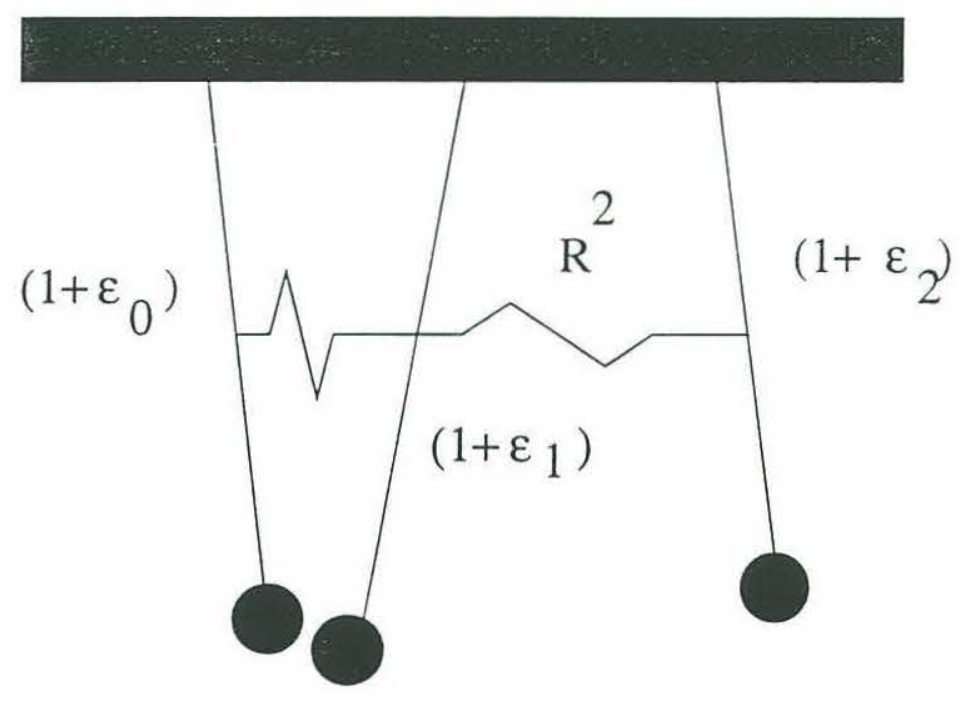

System of Three Coupled Pendula

Figure 2-5: System of three pendula 


\begin{tabular}{||c|c|c||}
\hline \hline Branch Point & $\epsilon_{1}$ & $\epsilon_{2}$ \\
\hline \hline (a) & $.0145 \pm .0268 i$ & .0101 \\
(b) & $.0043 \pm .0262 i$ & -.0099 \\
(c) & $.0099 \pm .0144 i$ & $-.0008+.0283 i$ \\
\hline \hline
\end{tabular}

Table 2.1: Three Root Coalescences

$$
\Delta\left(\lambda, \epsilon_{1}, \epsilon_{2}\right)=0
$$

where $\epsilon_{1}, \epsilon_{2}$ are complex disorder parameters. We can have a variety of eigenvalue coalescences. We could have two root coalescences. They are obtained as the solution to equation 2-68 and

$$
\frac{\partial \Delta}{\partial \lambda}=0
$$

We can either assign an arbitrary value to $\epsilon_{1}$ and solve for complex $\epsilon_{2}$ or vice versa. There are an infinite number of two root coalescence points. We could have a three root coalescence by adding the condition

$$
\frac{\partial^{2} \Delta}{\partial \lambda^{2}}=0
$$

We would then have to solve a set of complex nonlinear algebraic equations for the unknowns $\left(\lambda, \epsilon_{1}, \epsilon_{2}\right)$. The three root coalescence implies that three eigenvectors have large sensitivity on the nearby real axis while the two root coalescence implies that we have only two modes with significant sensitivity on the nearby axis providing the branch points are sufficiently close to the real axis. We plot projections of the complex lines of two root coalescences and three root coalescences on the real axes in figure 2-6. The three root coalescences lie close to where the two root coalescence lines approach each other.

We now consider two lines along which we provide eigenvector predictions using 




Figure 2-6: -: Two root coalescences. (a,b): Projection of three root coalescence points on real axis. 
(a) Good Jordan prediction, poor MPM prediction.
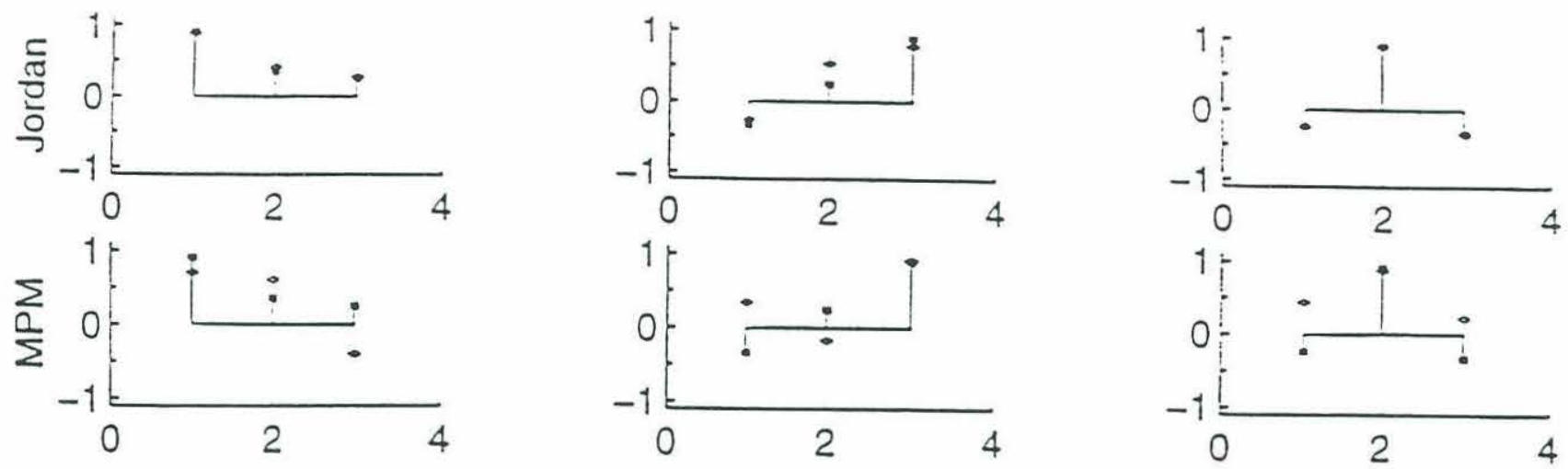

(b) Good Jordan prediction, poor IIPM prediction.


(c) Good MPM predictions, poor Jordan block predictions.
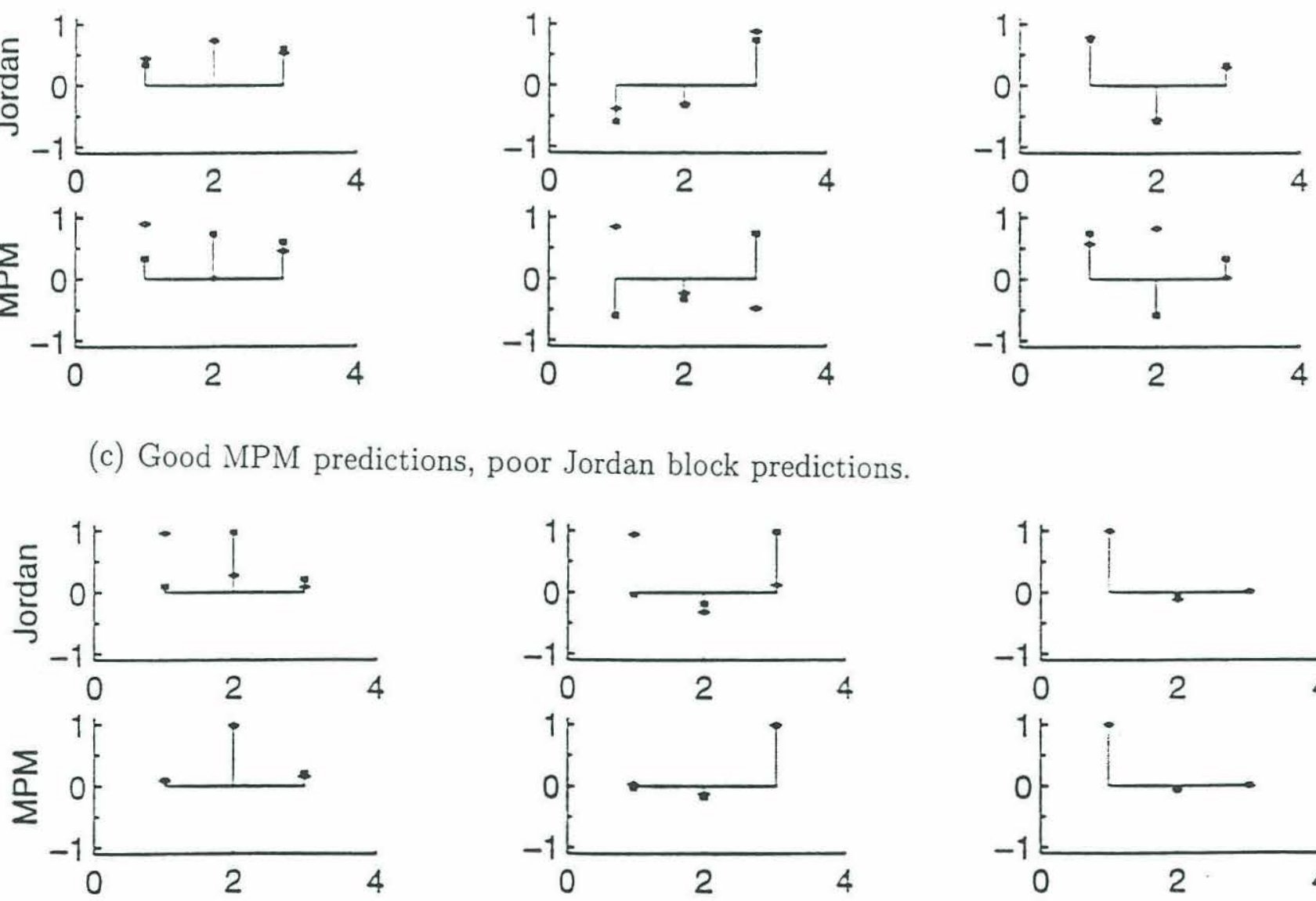
the Jordan block expansion and MPM at various points. The first line is $\epsilon_{1}=-\epsilon_{2}$. We provide plots at the points $P 1\left(\epsilon_{1}=-.01, \epsilon_{2}=.01\right), P 2\left(\epsilon_{1}=.01, \epsilon_{2}=-.01\right), P 3$ $\left(\epsilon_{1}=.06, \epsilon_{2}=-.06\right)$. The three points on this line are represented as triangles in figure 2-6. Point $P 1$ and $P 2$ are in the zone where the cube-root branch point effect is important while $P 3$ is in a zone where all the modes are localized and is also far away from all branch points. $P 1$ is on one side of the branch point (b) while $P 2$ is on the other side of the branch point while being sufficiently far away from the branch point to ensure the modes appear reasonably heavily localized. Two themes are developed using these three points. The first is that the Jordan block expansion performed well in a zone where all three modes are varying rapidly in response to disorder. The second point is that the edge of the predictive capabilities of the Jordan block expansion is such that modes in the entire range of intermediate localization are covered. In figure 2-7, we provide Jordan block expansion and MPM predictions at each point. The Jordan block expansions are performed about branch point (a) at point $P 1$ and branch point (b) at point $P 2$. In figure 2-7a, we provide Jordan block and MPM predictions at $P 1$. In figure $2-7 \mathrm{~b}$, we provide Jordan block expansion and MPM predictions at $P 2$. Finally, in 2-7c, we provide Jordan block and MPM predictions at $P 3$. These points start on one side of the origin, move to the other side and gradually enter a zone of large disorder. Points $P 1$ and $P 2$ are in a zone where the highest order Jordan block expansion performs well and the modes are moderately localized while point $P 3$ is in a zone where only the MPM performs well and all the modes are heavily localized. There is a gradual increase in localization in the modes when we pass from $P 1$ to $P 2$. The third point is associated with localization of all the modes. The Jordan block method which performs well at points $P 1$ and $P 2$ performs poorly at point $P 3$ while the MPM performs poorly at points $P 1$ and $P 2$ but performs well at point $P 3$.

The second line is along the coordinate $\epsilon_{1}=.06$ and predictions at two points $P 1^{\prime}$ and $P 2^{\prime}$ are plotted in figure 2-8. The two points on this line are represented by squares in figure 2-6. The theme developed in this figure is that there are lower order branch points (in this case, square-root branch points) in addition to the highest 
(a) Good Jordan prediction, poor MPM prediction.
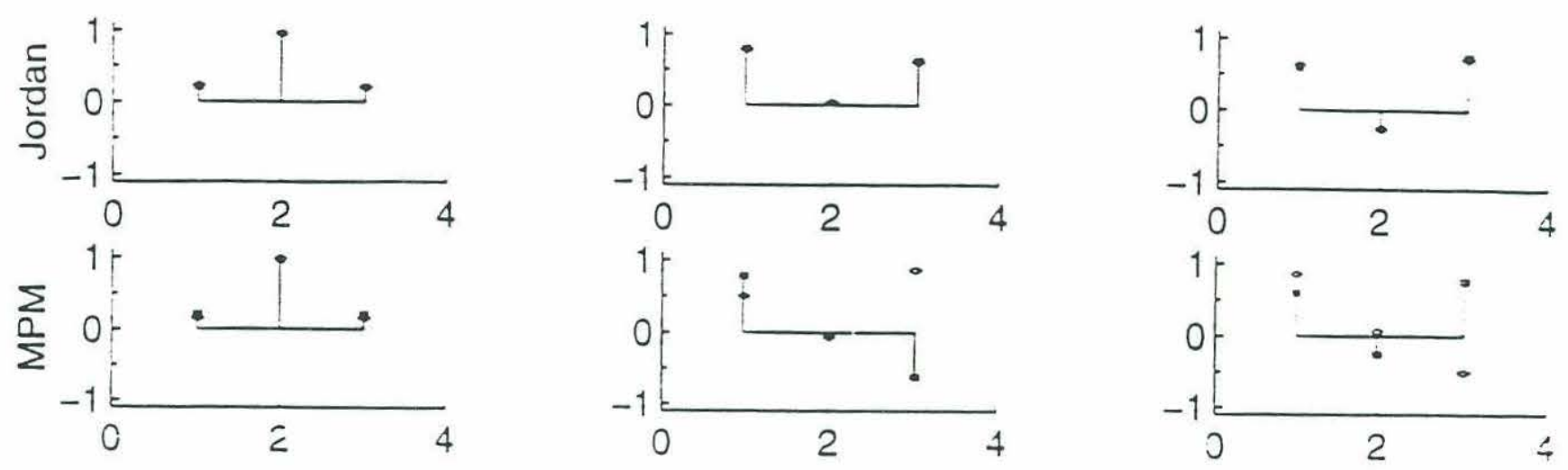

(b) Good MPM prediction, poor Jordan prediction.
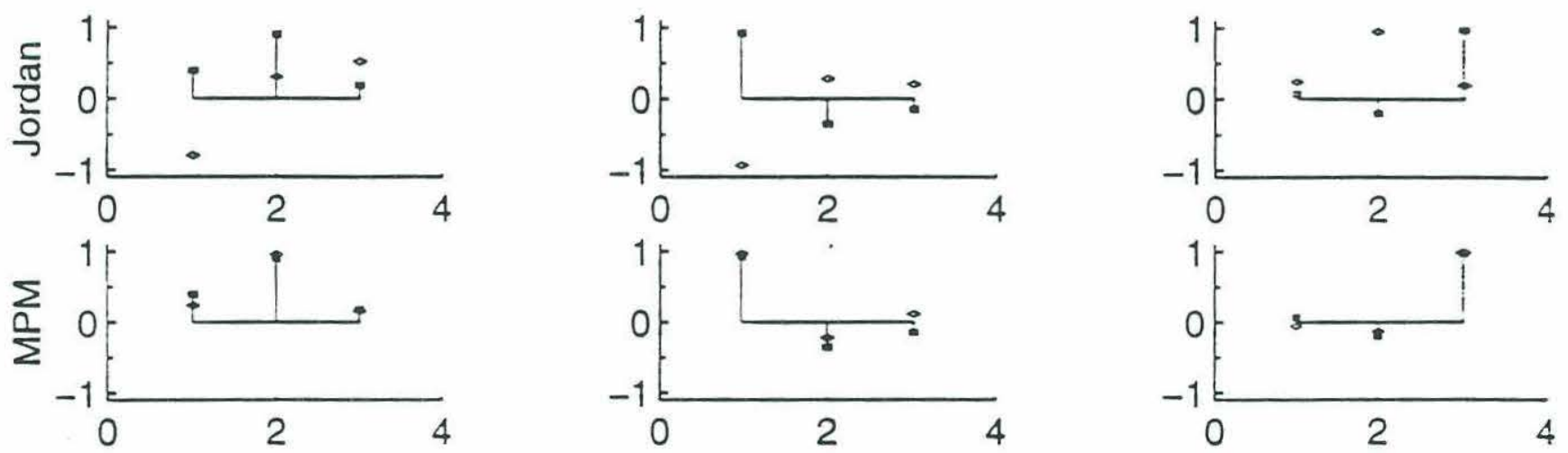

Figure 2-8: (a) : Jordan block and MPM predictions at $\left(P 1^{\prime}\right) \epsilon_{1}=0.06, \epsilon_{2}=$ .001. Expansion about square-root branch point at $\left(\epsilon_{1}=0.06, \epsilon_{2}=0.0001828+\right.$ $.004099 i)$. (b) : Jordan block and MPM predictions at $\left(P 2^{\prime}\right) \epsilon_{1}=.06 . \epsilon_{2}=.03$ $(\square$ : Exact Eigenvector. $\diamond$ : Predicted Eigenvector.) 
branch points and expansions about these branch points aid in providing analytical descriptions for modes in an intermediate state of localization. The modes are qualitatively different in appearance from the kind of modes we saw close to at cube-root branch point. Here one mode is localized and two have intermediate localization unlike near a cube-root branch point where all three modes displayed moderate localization. The points along this line which we consider are $P 1^{\prime}\left(\epsilon_{1}=0.06, \epsilon_{2}=.001,\right)$ and $P 2^{\prime}\left(\epsilon_{1}=.06, \epsilon_{2}=.03\right)$. The Jordan block expansion which we use is a two root coalescence expansion performed about the point $\epsilon_{2}=0.0001828+.004099 i$, and $\epsilon_{1}=0.06$. In sum, we have a wide variety of modes in various states of moderate localization which can be described well using Jordan block expansions about various order branch points.

We now construct some rules for questions like do we use the MPM or Jordan block expansion to determine the modes associated with a given point in the parameter space. If we use the Jordan block expansion, which order coalescence do we use and how do we select the correct branch point from the ones we have? We will discuss these questions here with emphasis on the three pendula problem and then the ten pendula problem.

We construct convergence zone diagrams for the MPM and Jordan block expansion for the three pendula system. We have two and three root coalescence branch points associated with this system. However the MPM which is an expansion about the uncoupled disordered state has a convergence zone only to the outermost branch points. The convergence zone for the MPM and Jordan Block Perturbation Method for a three pendula system are shown in figure 2-9. Since the complex conjugate square-root branch points straddle the real axis and are placed at a distance $\operatorname{Im}\left(\epsilon_{i}\right)$ on either side of the real axis, the MPM convergence zone is essentially the envelope formed by the lines $\operatorname{Re}\left(\epsilon_{i}\right) \pm \operatorname{Im}\left(\epsilon_{i}\right)$ where $\operatorname{Re}\left(\epsilon_{i}\right)$ and $\operatorname{Im}\left(\epsilon_{i}\right)$ are the real and imaginary coordinates of the two root coalescence branch point associated with the three pendula system. The convergence zone for the MPM is exclusively determined by the geometry of the branch points. Determining the zone of convergence for the Jordan block expansion is complicated because we have different Jordan block expansions. We have 
six possible three root coalescence Jordan block expansions and an infinite number of two root coalescence Jordan Block expansions. We can only talk in terms of a convergence envelope where either one or the other Jordan block expansion can be used. Hence, we provide an envelope convergence zone in which one or the other Jordan Block expansions would be valid. This is the region interior to the MPM convergence zone.

Let us assume we are in a zone of parameters where the Jordan block expansion is valid. There are altogether six cube-root branch points given in table 2-1. How do we choose the correct branch point to perform the expansion? Let us say we are considering a combination of parameters $\left(\epsilon_{1}, \epsilon_{2}\right)$. If all three modes display appreciable modal sensitivity close to this region of the parameter space, we can conclude we have to use a third order branch point expansion. The next question, is which of the three branch point do we use as our expansion point ? The branch point which is the closest to the point in question is used as the branch point for expansion. It is very interesting that if we take a sample of points ranging from $\left|\epsilon_{1}\right|<.02$ and $\epsilon_{2}<.02$, and compute the closest branch point to each point in the disorder space, only branch points (a) or (b) contribute to the solution set. Branch points (c) are slightly deeper in the complex plane but are situated so that they are further away (relative to (a) and (b)) from the point considered in the disorder parameter space. When we have larger values of disorder $\epsilon_{1}, \epsilon_{2} \simeq O\left(R^{2}\right)$ or greater, we find two situations : the first is that where all three modes are localized and the second is where only one mode is localized. The case where two modes are extended is typically a case where two modes display appreciable sensitivity. In the first case, we use an MPM expansion while in the second case we use a square-root branch point expansion. We determine the point of expansion by determining the closest two root coalescence line. These ideas are applicable to larger problems. We would then examine $m$ modes for their sensitivity and extended nature. We would subsequently use a Jordan block size $m$ expansion. For this three pendula system that we are studying, we would thus use a Jordan block size three or size two expansion. The details of the Jordan block size three expansion are shown in appendix $\mathrm{C}$. The perturbation expansion for a size three 


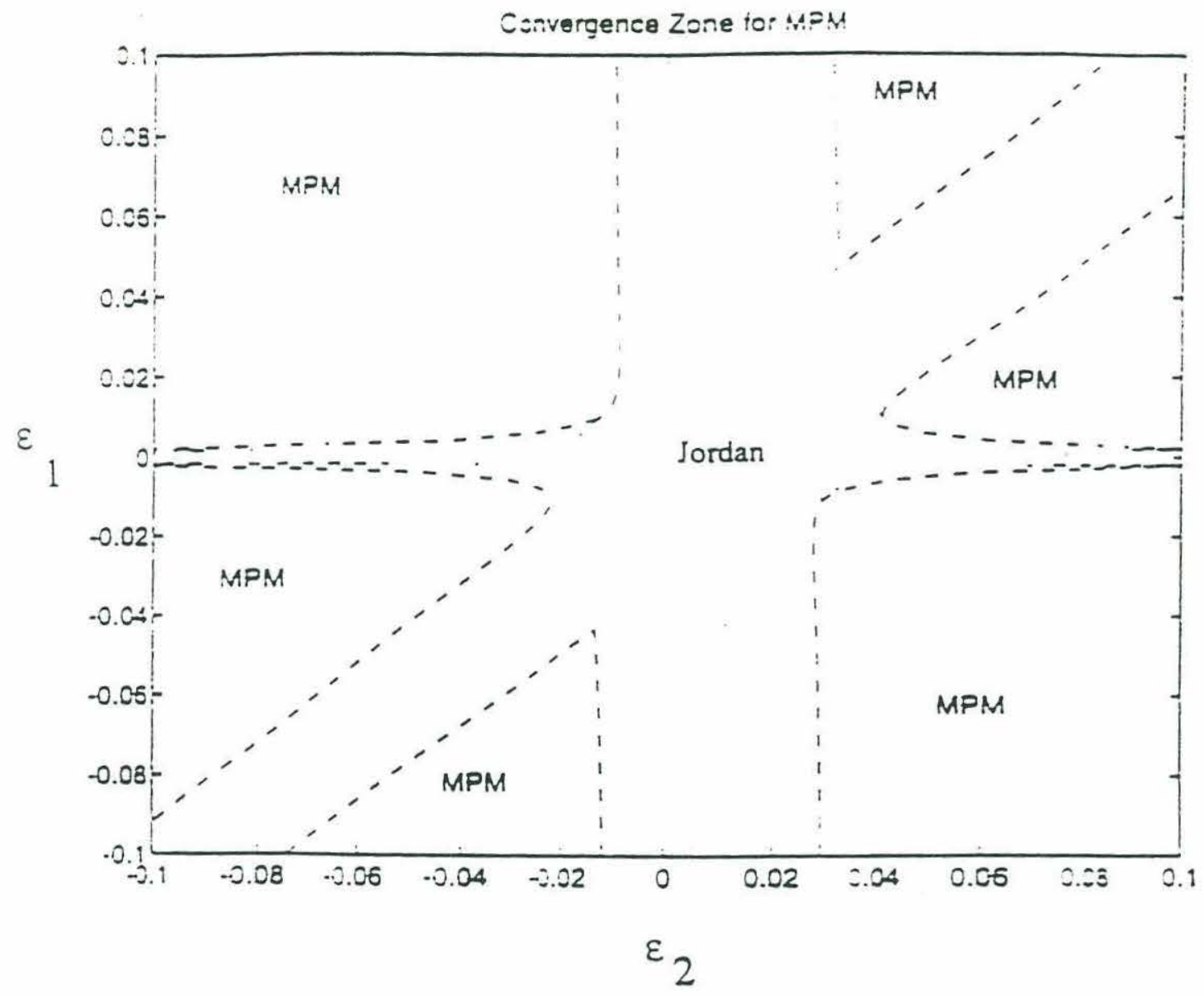

Figure 2-9: Convergence zone for MPM and envelope of convergence for Jordan block expansion 
Jordan block expansion is of the form

$$
\begin{gathered}
{\left[K_{0}+\delta K+\delta^{2} K\right]\left\{x_{01}+\delta^{\frac{1}{3}} x+\delta^{\frac{2}{3}} x+\delta x+\ldots\right\}=} \\
\left(\lambda_{0}+\delta^{\frac{1}{3}} \lambda+\delta^{\frac{2}{3}} \lambda+\delta \lambda+\ldots\right)\left\{x_{01}+\delta^{\frac{1}{3}} x+\delta^{\frac{2}{3}} x+\delta x+\ldots\right\}
\end{gathered}
$$

where $x_{01}$ is the eigenvector associated with a Jordan block of size three.

Different branch point expansions are valid in different zones of the parameter space. In figure 2-10, we show eigenvector predictions at two points (B1) and (B2) using two different Jordan block size three expansions. Point (B1) is at $\epsilon_{1}=.005$ and $\epsilon_{2}=.01$. Point (B1) is closer to branch point (a) but further away from branch point (b). Point (B2) is closer to branch point (b) but further away from branch point (a). We provide predictions close to branch point (a) in figure 2-10 a and predictions close to branch point (b) in figures 2-10 b. Clearly the branch point (b) expansion fares poorly in comparison with the branch point (a) expansion in 2-10 a. It is clear that this point is very close to branch point (a) and far away from branch point (b) and hence the reason for one expansion performing better than the other. Point (B2) is $\epsilon_{1}=.005, \epsilon_{2}=-.01$. The branch point (a) expansion in figure $2-10 \mathrm{~b}$ fares poorly compared to the branch point (b) expansion in figure 2-10 b in this zone of the parameter space. It is obvious that in moving from point (B1) to point (B2), we have gone from a point where a branch point (a) expansion performed better to a point where a branch point (b) expansion performed better.

In sum, if we examine the MPM convergence zone from figure 2-9, it is clear that in the zone where the disorder $\epsilon_{i} \simeq O\left(R^{2}\right)$, we can use the Jordan block expansion. In the zone where $\left|\epsilon_{i}\right|>>R^{2}$, we can use the MPM if we are clear of the square-root branch points. It is also clear that the modes change very dramatically in this small zone of parameters where Jordan block expansions are applied as seen in figure 2-7, 2-8 and 2-10. Hence the Jordan block expansion is useful in zones where there is dramatic variation of the mode shapes. 
(a) Good Jordan block (a) and bad Jordan block (b) prediction.
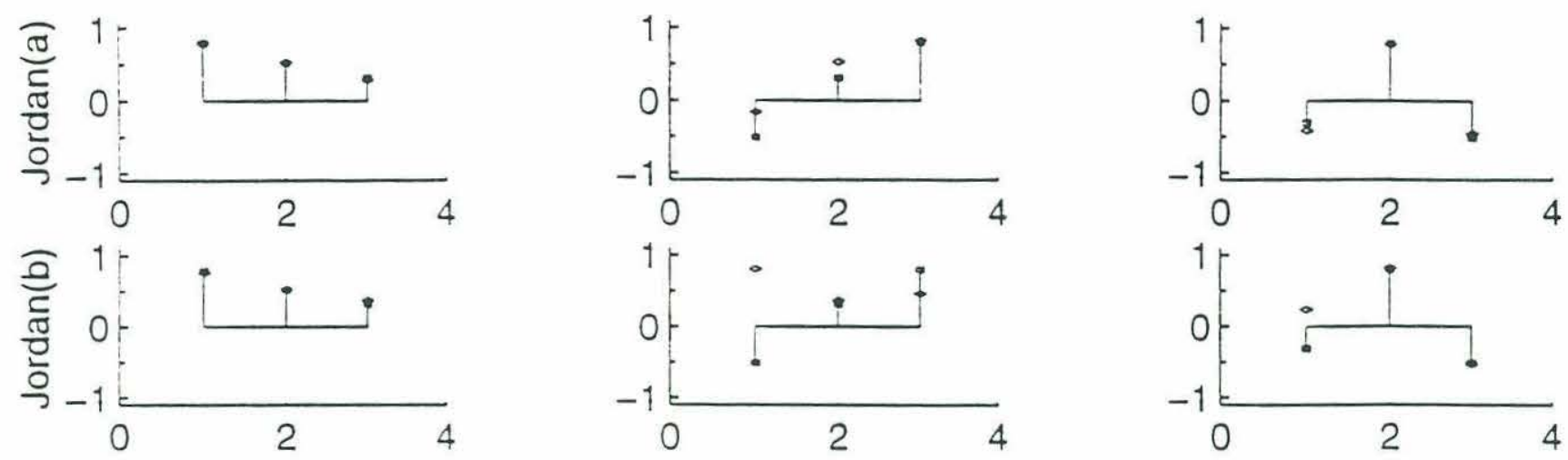

(b) Good Jordan block (b) and bad Jordan block (a) prediction.
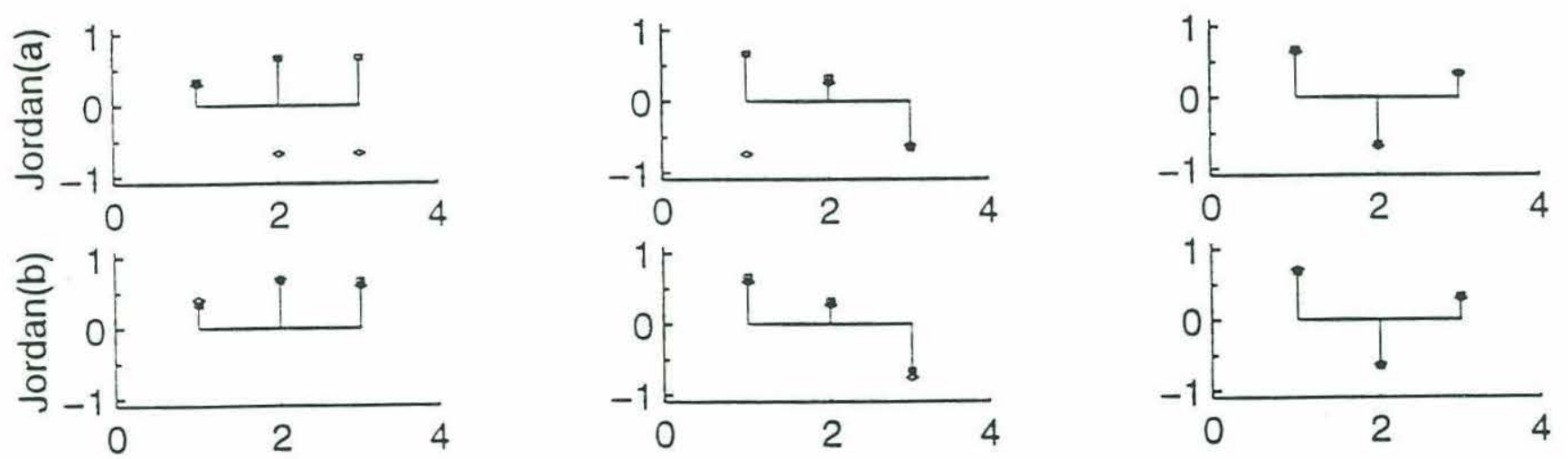

Figure 2-10: (a) : Jordan block (a) and Jordan block (b) predictions at $B 1$ $\left(\epsilon_{1}=.005, \epsilon_{2}=.01\right)$. (b) : Jordan block (a) and Jordan block (b) predictions at $B 2\left(\epsilon_{1}=.005, \epsilon_{2}=-.01\right)$ ( $\square$ : Exact Eigenvector, $\diamond:$ Predicted Eigenvector.) 


\subsubsection{Prediction of modes for ten pendula using lower order coalescence expansion}

We have so far been looking mostly at eigenvector predictions where the Jordan block is the same size as the stiffness matrix. For example in an $n$ pendula system, we have a Jordan block of size $n$. We next examine a case where the Jordan block is of size $m$ where $m<n$. In such a case, we would have an $m$ th root branch point in the eigenvalue-disorder relation. If we apply a similarity transform, we would get

$$
Y^{H}[K][X]=\left[\begin{array}{ccccccccccc}
\lambda_{0} & 1 & 0 & 0 & 0 & 0 & 0 & 0 & 0 & 0 & 0 \\
0 & . & 1 & 0 & 0 & 0 & 0 & 0 & 0 & 0 & 0 \\
0 & 0 & . & 1 & 0 & 0 & 0 & 0 & 0 & 0 & 0 \\
0 & 0 & 0 & . & 1 & 0 & 0 & 0 & 0 & 0 & 0 \\
0 & 0 & 0 & 0 & \lambda_{0} & 0 & 0 & 0 & 0 & 0 & \\
0 & 0 & 0 & 0 & 0 & \lambda_{m+1} & 0 & 0 & 0 & 0 & 0 \\
0 & 0 & 0 & 0 & 0 & 0 & \lambda_{m+2} & 0 & 0 & 0 & 0 \\
0 & 0 & 0 & 0 & 0 & 0 & 0 & . & 0 & 0 & 0 \\
0 & 0 & 0 & 0 & 0 & 0 & 0 & 0 & . & 0 & 0 \\
0 & 0 & 0 & 0 & 0 & 0 & 0 & 0 & 0 & . & 0 \\
0 & 0 & 0 & 0 & 0 & 0 & 0 & 0 & 0 & 0 & \lambda_{n}
\end{array}\right]
$$

When we apply a Jordan block expansion about this point, we would be using a hybrid of two methods. The first method is the Jordan block expansion while the second method is that used for matrices with distinct eigenvalues (Courant and Hilbert [11]). The $m$ eigenvectors associated with the Jordan block are expanded in a series in powers of the $m$ th root of the disorder parameter. The other eigenvectors can only be expanded in integral powers of the disorder parameter. We examine the expansion associated with a Jordan block of size two as a special case to illustrate a few principles. The eigenvectors associated with the branch point are expanded using the equation similar to that for the square-root branch point. 


$$
\left[K_{0}+\delta K+\delta^{2} K+\ldots\right]\left\{x_{01}+\delta^{\frac{1}{2}} x+\delta x+\ldots\right\}=\left(\lambda_{0}+\delta^{\frac{1}{2}} \lambda+\delta \lambda+\ldots\right)\left\{x+\delta^{\frac{1}{2}} x+\delta x+\ldots\right\}
$$

The eigenvector not associated with the branch point can be expanded using a Taylor series.

$$
\left[K_{0}+\delta K+\delta^{2} K+\ldots\right]\left\{x_{0 j}+\delta x+\delta^{2} x+\ldots\right\}=\left(\lambda_{0}+\delta \lambda+\delta^{2} \lambda+\ldots\right)\left\{x_{0 j}+\delta x+\delta^{2} x+\ldots\right\}
$$

$x_{0 j}$ is the eigenvector not associated with the branch point. There is clearly a big difference between the expansions used for the two types of eigenvectors. At each order, the branch point perturbation is expanded using equation 2-23.

The issue of interest is to determine the nonzero coefficients in the expansions for the eigenvectors associated with the branch point. The generalized eigenvector is the eigenvector number 1 . Since the eigenvector is perturbed away from eigenvector 1, all the coefficients associated with that eigenvector are zero for all orders. Many of the other eigenvector coefficients are also zero making the calculation relatively simple. We will refer to this method as a hybrid Jordan block expansion to distinguish it from the Jordan block expansion.

We provide lower order coalescence expansion predictions for moderately localized modes of a system of ten coupled pendula. We could have used a ten root coalescence expansion but determining the ten root coalescence coordinates is difficult because as the order of the characteristic polynomial increases, the roots of the polynomial become increasingly sensitive to small perturbations to the coefficients of the polynomial. So truncation errors can cause us to make errors in our estimate of the branch point. For example if we calculate the $n$ root coalescence coordinate correct to $p$ decimal places, and then substitute the disorder values back into the stiffness matrix, we would have errors of $O\left(10^{-\frac{p}{n}}\right)$ in the eigenvalues due to the $n$ root dependence of the eigenvalue on disorder. If $n=10$ and $m=8$, we would only be able to obtain $10^{-\frac{4}{5}}$ accuracy which is not even one decimal place accuracy. There is another important effect of computing higher order coalescences. The coefficients 
of the characteristic polynomial are polynomial function of $R^{2}$. For larger systems, the polynomial coefficients could be function of high powers of $R^{2}$. If the coupling is a small number like .01, we would get round-off errors affecting the coefficients of the polynomial. For example, if we have coefficients which are functions of $\left(R^{2}\right)^{9}$, we would have, due to finite precision effects, lost significant digits associated with these coefficients if we working in double precision. $n$th root amplification of this error would result in $O(.1)$ errors in the roots of the equation. The Jordan block vectors (especially the generalized eigenvectors) are very sensitive to small errors in calculation of the complex branch point coordinates. Since we cannot compute higher order coalescences accurately, we cannot provide analytical prediction of modes for large systems of pendula for cases where the disorder is very small, and where all, or a large number of modes show appreciable modal sensitivity. Even if we were able to calculate the complex coordinates for eigenvalue coalescence, we would require a large number $(2 n)$ of terms to obtain reasonable eigenvector predictions since we are expanding in powers of $\epsilon^{\frac{1}{n}}$ where $\epsilon$ is the disorder parameter. As an example of the computational needs, for $n=20$, we would require 40 terms if we wish to expand the series to order $\epsilon^{2}$.

We follow the techniques described earlier in performing hybrid Jordan block expansions. We provide a Jordan block expansion about the disorder parameter $\epsilon_{9}=-.2643-.4866 i$. This is a point of two root coalescence. We provide predictions of the two modes which are linked with the two root coalescence. We cannot use the MPM because adjacent modes have zero disorder. We use the CPM because it is is the only expansion which could provide predictions in this zone of the parameter space. We again traverse two points along a line. The first point is $t 1$ with coordinate $\epsilon_{1}=0, \epsilon_{2}=0, \ldots, \epsilon_{8}=0, \epsilon_{9}=-.2643$, and the second $\mathrm{t} 2$ with coordinate $\epsilon_{1}=0, \epsilon_{2}=$ $0, \ldots=\epsilon_{8}=0, \epsilon_{9}=-.0043$. The first point is close to the two root coalescence. The second point is close to the periodic state. If we could use a 10 root coalescence expansion, we would get excellent predictions for the second case. However, due to our numerical constraints we will only explore the predictive capabilities of a two root coalescence expansion. Point $\mathrm{t} 1$ and $\mathrm{t} 2$ lie on this straight line. The predictions are 
shown in figure 2-11. The Jordan Block method (2-11a) works far better than the CPM (2-11b) at the point t1. The CPM works better at t2(2-11d) than the Jordan expansion(2-11c). The CPM is useful only for modes which are almost periodic. This is obvious by examining the modes predicted by the CPM. The MPM is far more useful for examining heavy localization than the CPM. The Jordan Block method is the third alternative in the analytical study of localized modes.

\subsection{Conclusions}

We provide a new method in the analytical description of localized mode shapes thereby extending the existing predictive capabilities of perturbation techniques. The MPM and CPM expansions are useful for describing lightly and heavily localized modes over a vast range of disorder parameters. The intermediate and relatively smaller range of parameters corresponds to moderately localized modes. The mode shapes in this range of parameters display maximum sensitivity in their transition from periodic to localized modes. We demonstrate that the usage of different order Jordan block expansions is useful in describing different modes in this intermediate range. We also provide convergence zones associated with different expansions. The drawback of this method associated with numerical limitations, (i.e. the inability to calculate the branch points associated with higher order coalescences) is discussed. 


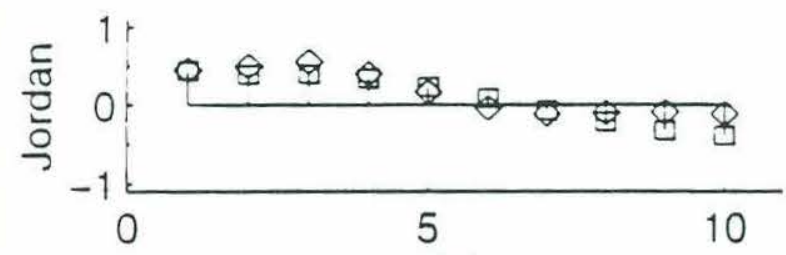

(a)

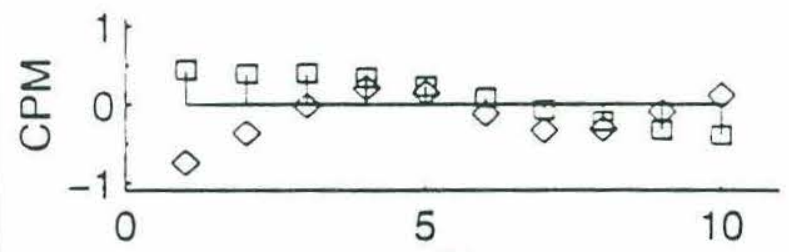

(b)

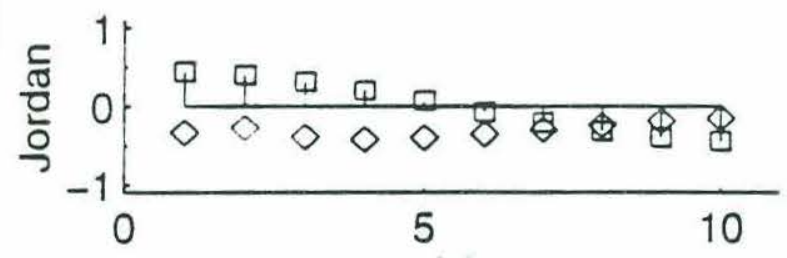

(c)

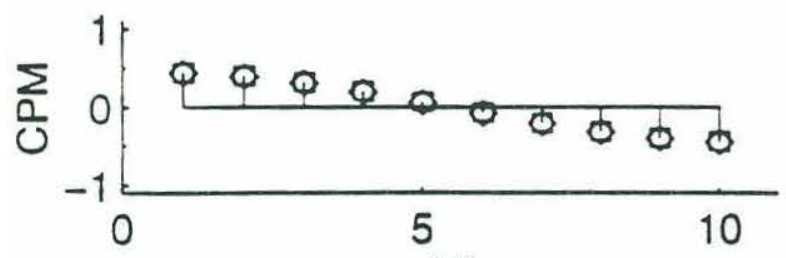

(d)
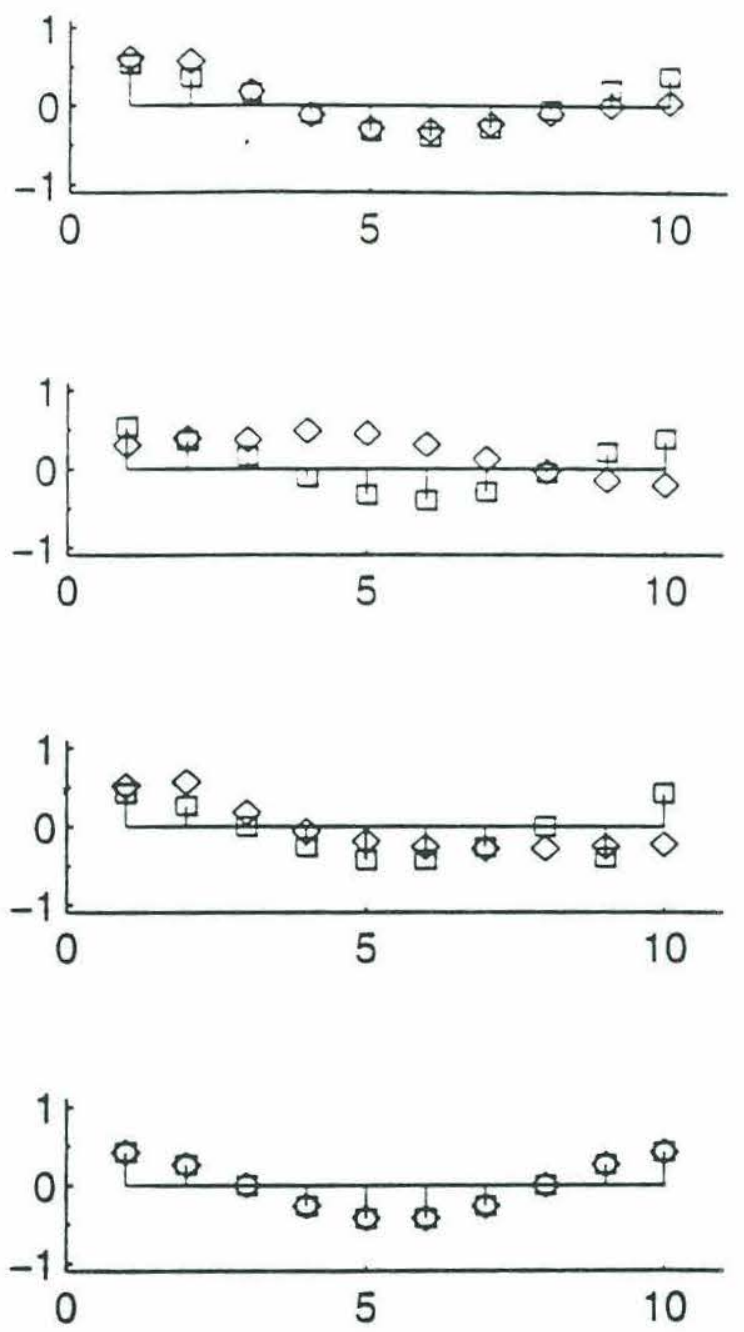

Figure 2-11: Jordan Block expansion (a) and CPM (b) predictions for ten pendula model at $\epsilon_{9}=-.2643$. Jordan block expansion (c) and CPM (d) expansion at $\epsilon_{9}=$ .0043. $\square$ : Exact Value, $\diamond$ : Predicted Value. 


\section{Chapter 3}

\section{Investigation of the effects of the strength and location of the branch points on localization}

\subsection{Introduction}

We have in the previous chapter found the general trend that the modes in an intermediate state of localization displayed large sensitivity to disorder. Jordan block expansions about these branch points were useful in describing modes in an intermediate state of localization. However we have only talked in very general terms about the sensitivity of modes without specifying any quantitative measures for localization. We introduce quantitative measures for modal sensitivity and localization in this chapter and then use these measures to study localization.

Triantafyllou and Triantafyllou [39] performed asymptotic analyses to demonstrate that branch points in the frequency-disorder relation were responsible for the large sensitivity seen in localization. The term "geometry" refers to the properties of points and surfaces. Depending on the number of pendula and disorder parameters, we could have either a simple branch point in the eigenvalue-disorder relation or more complicated surfaces (when the term branch-surface would be more appropri- 
ate). The branch point geometry is responsible for the large sensitivity seen in systems exhibiting localization. The strength of the branch point(referring to the order of the branch point in the mode-disorder relation) and the location of the branch point with respect to the real axis are the main geometric properties which control the modal sensitivity and localization. The precise distribution of these branch points which have varying strengths (determined by the order of the branch point) and distances from the real axis is responsible for the varying levels of sensitivity to different combinations of disorder. A very rich analogy exists with the field of electromagnetism. The electromagnetic field at a point is determined by the distribution of singularities of varying strengths like poles, dipoles, quadrupoles etc. and their distance from the point of the space in question. In complete analogy, we have a set of singularities (albeit weaker, being branch points) of varying strengths (the $n$th root dependence on disorder where $n$ is the variable order of the branch point) and varying distances (depending on their positions in the complex plane) from the point of the disorder parameter space in question, defining the modal sensitivity (or alternately the localization) at the point. One special feature of localization which does not exist in the electromagnetic analogy is that $n$ modes show appreciable sensitivity if the disorder parameter combination is close to an $n$ root coalescence (Refer Chapter 2, Jordan Block Perturbation for a Jordan block of size $n$ ).

This chapter explores the complex implications on mode localization due to the distribution of these branch points in the complex plane. Sensitivity is connected to localization because the integral of the modal sensitivity is related to localization. So understanding sensitivity helps us understand a number of problems in localization. One problem which will be resolved here is the observation by Hodges and Woodhouse([19]) that similar combinations of disorder which share the same statistical properties (we examine the mean square disorder here) result in different levels of localization. Another problem which we look at is the utilization of this knowledge of the distribution of strength and location of branch points to determine directions to search parameter combinations which have minimal mean square disorder. We also study the conflicting effects of the two geometric properties i.e. the strength and 
location of the branch point by examining some unusual configurations. Modal sensitivity of configurations which are associated with high strength branch points can sometimes be lower than those associated with lower strength branch points owing to differing locations of the branch points.

Obviously the presence of damping would alter the characteristic polynomial and alter the positions of the branch points in the complex plane. We neglect damping from the analysis because the effect of damping is not very relevant in the study of localization.

\subsection{Two pendula example}

We utilize modal sensitivity and localization factor definitions from Appendix A throughout to quantify localization and modal sensitivity. The modal sensitivity for a system dependent on only one parameter $\epsilon$ is determined by the

$$
Q(q, \epsilon)=\frac{\operatorname{Lim}}{\epsilon^{\prime} \rightarrow 0} \frac{\left|q\left(\epsilon+\epsilon^{\prime}\right)-q(\epsilon)\right|}{\epsilon^{\prime}}
$$

If the system is dependent on two parameters say $\epsilon_{1}$ and $\epsilon_{2}$, the modal sensitivity would actually represent a partial modal derivative. The total modal derivative or modulus of modal sensitivity would be

$$
Q(q)=\sum_{j=1}^{j=2} Q\left(q, \epsilon_{j}\right)^{2}
$$

The localization factor which is also defined in Appendix A is a measure of the exponential decay associated with the mode shape. It is based on the exponential decay associated with modes displaying heavy localization. The constant of exponential decay is assumed to be the localization factor. For modes displaying light localization, we do not usually witness a clear exponential decay and hence an exponential curve is fitted through the modal amplitudes to give a value for the localization factor (see Chapter 2 for details).

Asymptotic analyses performed by Triantafyllou and Triantafyllou [39] showed 
that the cause of the large modal sensitivity seen in localization was the presence of branch points in the mode-disorder relation. For a system with $n$ disorder parameters, the $m$ th root branch points could be determined by solving the equations

$$
\Delta\left(\lambda, \epsilon_{1}, \ldots \epsilon_{n-1}\right)=|K-\lambda I|=0
$$

and

$$
\frac{\partial^{i} \Delta}{\partial \lambda^{i}}=0
$$

where $1 \leq i \leq m-1$ and $m \leq n$.

Here $K$ is the stiffness matrix associated with a system of $n$ disordered pendula and $\epsilon_{i}(1 \leq i \leq n)$ are the disorder parameters introduced into each pendulum. The stiffness matrix as described in Chapter 2 is

$$
[K]=\operatorname{Tridiag}\left[-R^{2} \frac{\left(1+\epsilon_{j-2}\right)}{\left(1+\epsilon_{j-1}\right)} ; \frac{1}{\left(1+\epsilon_{j-1}\right)}+\left(2-\delta_{j, n}-\delta_{j, 1}\right) R^{2} ;-R^{2} \frac{\left(1+\epsilon_{j}\right)}{\left(1+\epsilon_{j-1}\right)}\right]
$$

where $1 \leq j \leq n$. The notation $\operatorname{Tridiag}\left(\alpha_{j}, \beta_{j}, \kappa_{j}\right)$ designates a tridiagonal matrix with $\alpha_{j}$ being the element of the lower diagonal ( $j$ th row, $(j-1)$ th column), $\beta_{j}$ is the element on the main diagonal (jth row, jth column), and $\kappa_{j}$ is the element on the upper diagonal ( $j$ th row, $(j+1)$ th column). By definition $\alpha_{1}=\kappa_{n}=0 . \delta_{i, j}$ is the Kronecker Delta function.

Consider a system of two coupled pendula with disorder $\epsilon$. Consider in figure 3-1 the modal sensitivity plotted as a function of disorder. This is done for three values of the coupling parameter. Clearly, the peak is not centered at zero disorder and the off-centered nature of the peak becomes more pronounced as we increase coupling. Conventional perturbation techniques(Pierre and Dowell [27]) indicate that the main cause of the large modal sensitivity is the closely spaced nature of the eigenvalues and that the peak modal sensitivity is at the point where the difference in the eigenvalues is minimum i.e. the point of zero disorder. This graph would seem to indicate the close eigenvalue spacing may not be the reason for the large modal sensitivity since the peak is not at the point of zero disorder. The reason for the off-centered peak can 


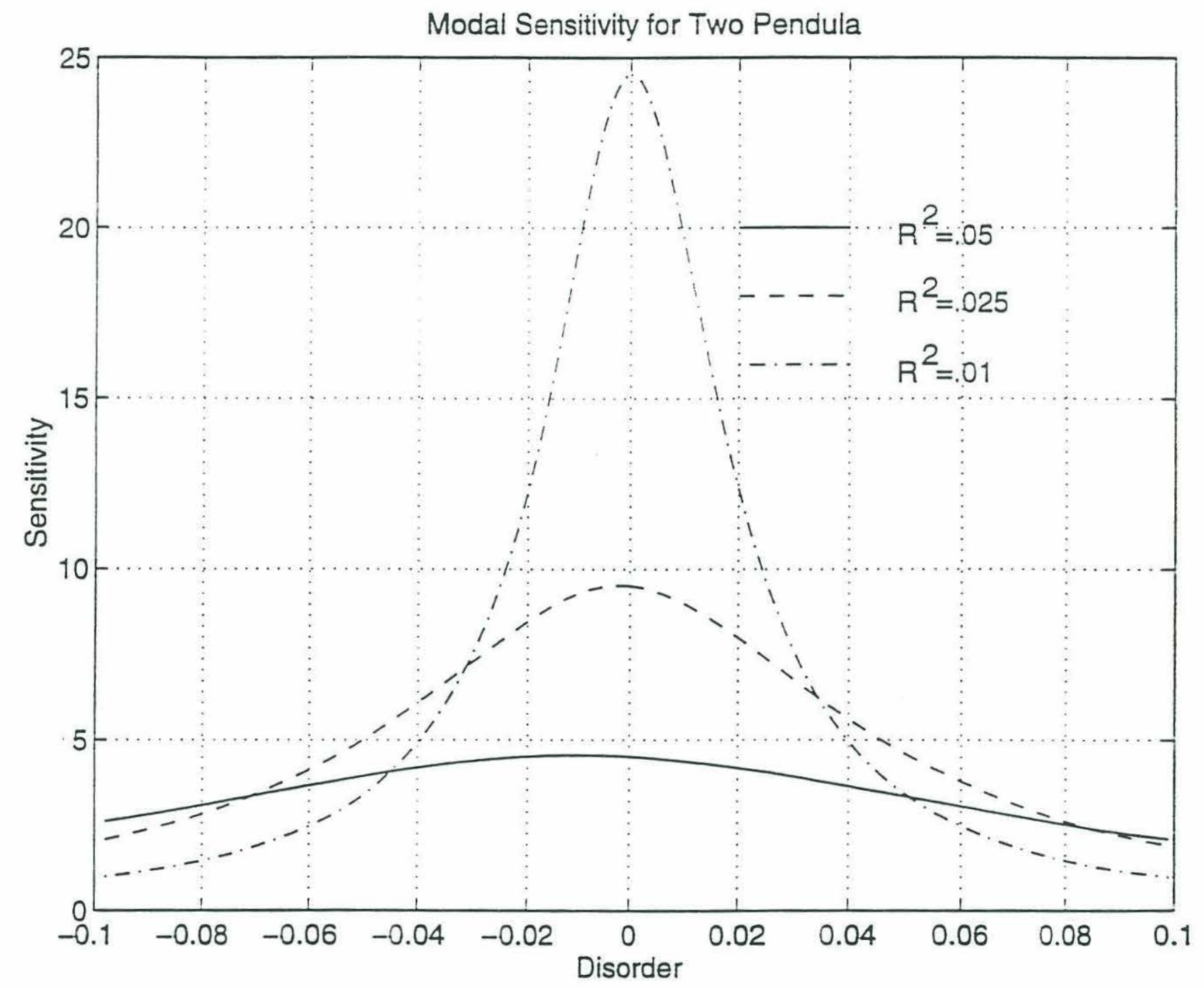

Figure 3-1: Modal Sensitivity as a Function of Disorder for $R^{2}=.01, R^{2}=.05$, and $R^{2}=.1$ 
be clearly understood by inspecting the expression for the branch point in equation $3-5$.

$$
\epsilon_{0}= \pm \frac{2 R^{2} i}{\left(1 \mp 2 R^{2} i\right)}
$$

There is a real negative part associated with the branch point coordinate. Its magnitude increases with coupling. So we should see a leftward shift in the peak of the modal sensitivity curve with an increase in coupling. Of course there is a decrease in the peak sensitivity with the increase in coupling because of the rapid approach of the branch point towards the real axis. See figure 3-2 for the variation of position of the branch point in the complex plane as a function of the disorder.

We also provide a plot of the variation of the localization factor versus disorder for a two pendula case in figure 3-3. The localization factor plot is also slightly asymmetric indicating a path skirting the branch point would result in larger change and quicker transition to localized state (i.e. the $\epsilon<0$ contour) as opposed to the $\epsilon>0$ contour which results in a slower transition to localized state. This seems reasonable because localization is proportional to the area under the modal sensitivity curve. Moving along the $\epsilon<0$ contour results in our covering greater area under the modal sensitivity curve as opposed to moving along the $\epsilon>0$ curve where far less area under the modal sensitivity curve was covered. Already an important fact has emerged. If our interest is to induce a certain level of localization in the modes of the structure, some combinations are more effective than others. Here, to ensure a localization factor of $\gamma=1.6$, we would have to pick a disorder magnitude $\epsilon$ slightly less than .05 if we considered negative disorder whereas if we considered positive disorder, we need a disorder magnitude slightly greater than .05 .

\subsection{Three Pendula Example}

We now consider a system of three pendula with disorder parameters $\left(\epsilon_{1}, \epsilon_{2}\right)$. We could either have square-root or cube-root type branch points in the mode-disorder relation. The cube-root branch points can be obtained by solving equations 3-1 and 


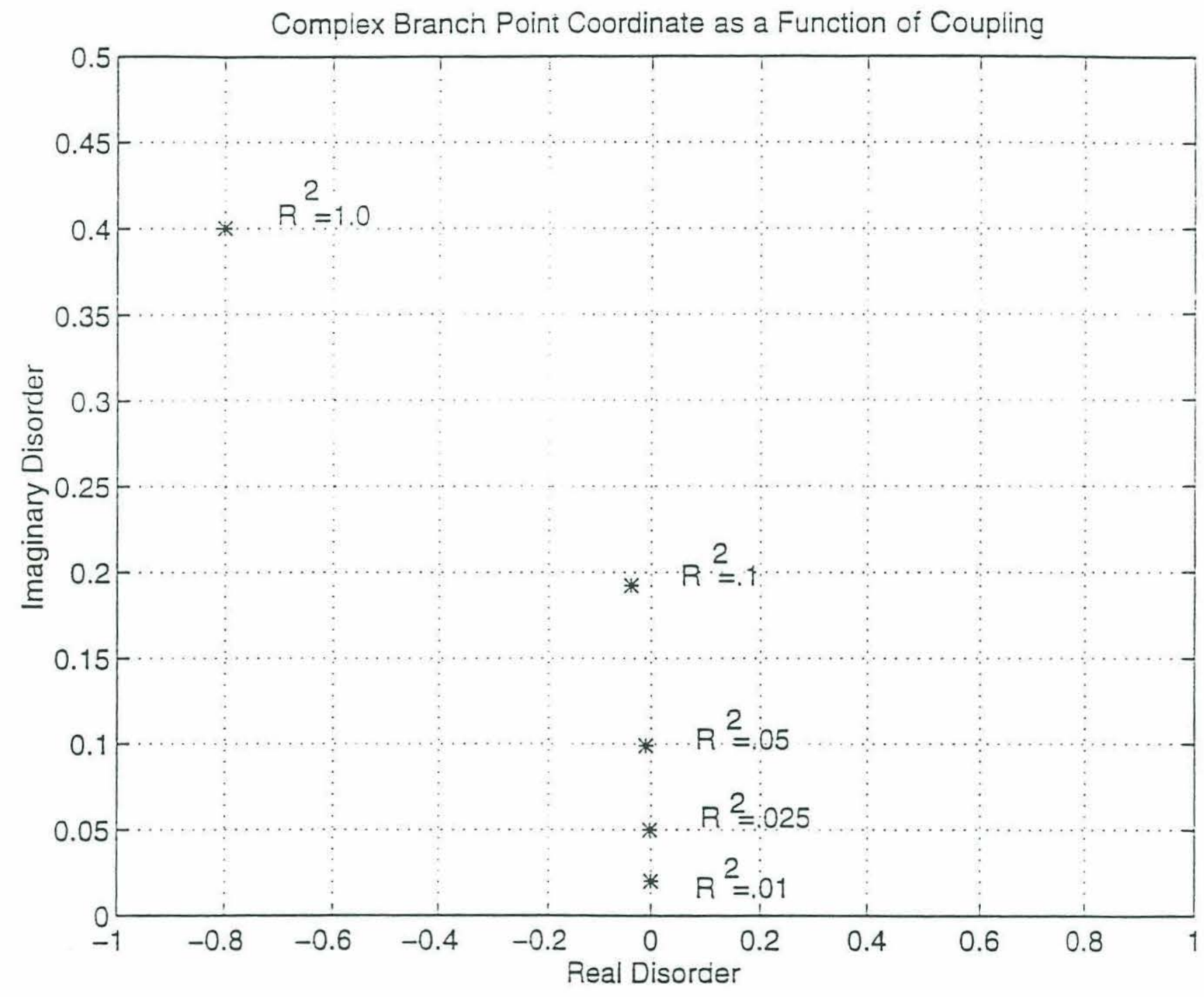

Figure 3-2: Position of branch point versus disorder 
Two Penduia : Localization vs. Disorder

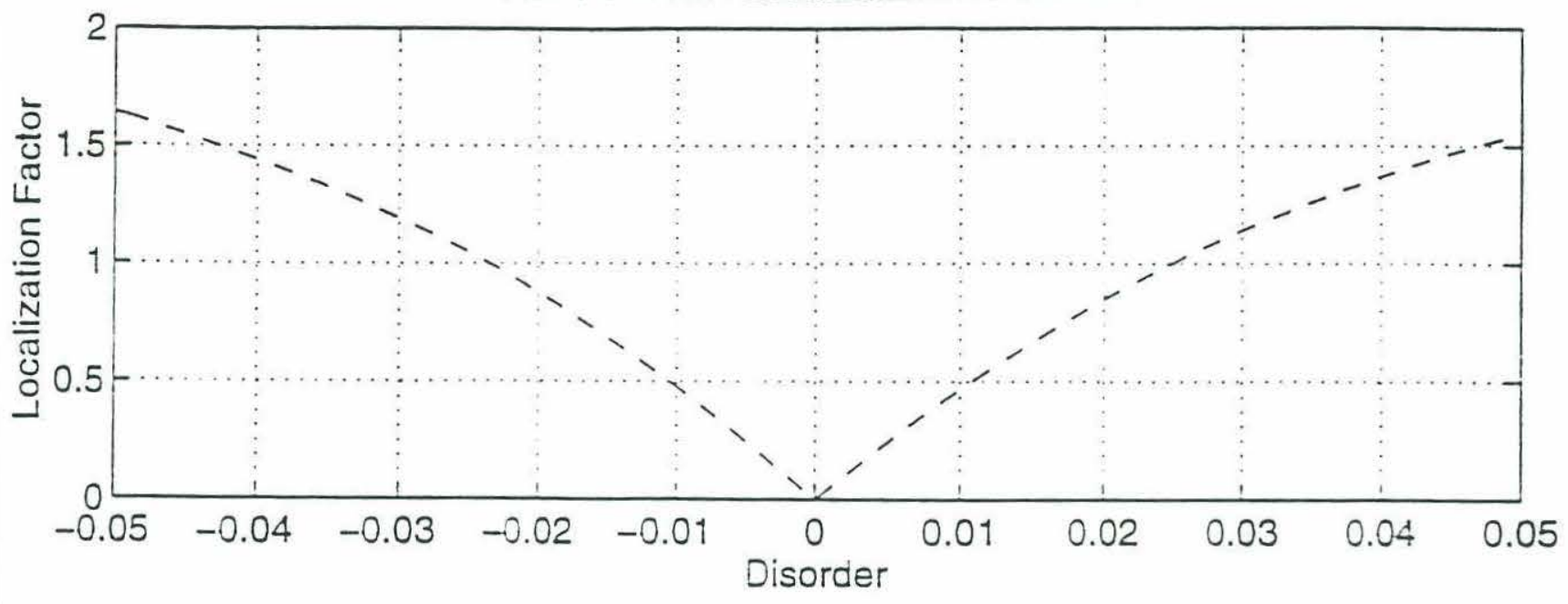

Figure 3-3: Localization factor versus disorder

3-2 with $m=2$. We could also have square-root type branch points in the modedisorder relation. If we solve equations 3-1 and 3-2 with $m=1$, we could solve for square-root branch points. As we have remarked in chapter 2, we have only two equations and three variables $\lambda, \epsilon_{1}$ and $\epsilon_{2}$ as variables. The only way to ensure unique solutions for equations 3-1 and 3-2 is by arbitrarily fixing one parameter say $\epsilon_{1}$ and solving for $\lambda$ and $\epsilon_{2}$ as unknowns. We could do the reverse i.e. fix $\epsilon_{2}$ and solve for the unknowns $\lambda$ and $\epsilon_{1}$. The next question is what arbitrary value do we fix for $\epsilon_{1}$ for the first case. We could permit $\epsilon_{1}$ to vary over the entire field of complex numbers. But we should pick that point on this surface which exerts maximum influence on the real axis. A reasonable argument is that we only permit $\epsilon_{1}$ to assume real values. $\epsilon_{2}$ and $\lambda$ are complex. If this choice is correct, we should expect to see the square-root branch point lines run parallel to the modal sensitivity lines. In figure 3-4, we proride plots of the modal sensitivity $Q\left(q_{i}, \epsilon_{2}\right)$ superposed on the projections of these branch points on the axis of real disorder for all three modes of the system. There are two square-root branches which are plotted in figure 3-4 and these lines run parallel to the ridges. We will name the one on the left as branch B1 and the one on the right branch B2. The 

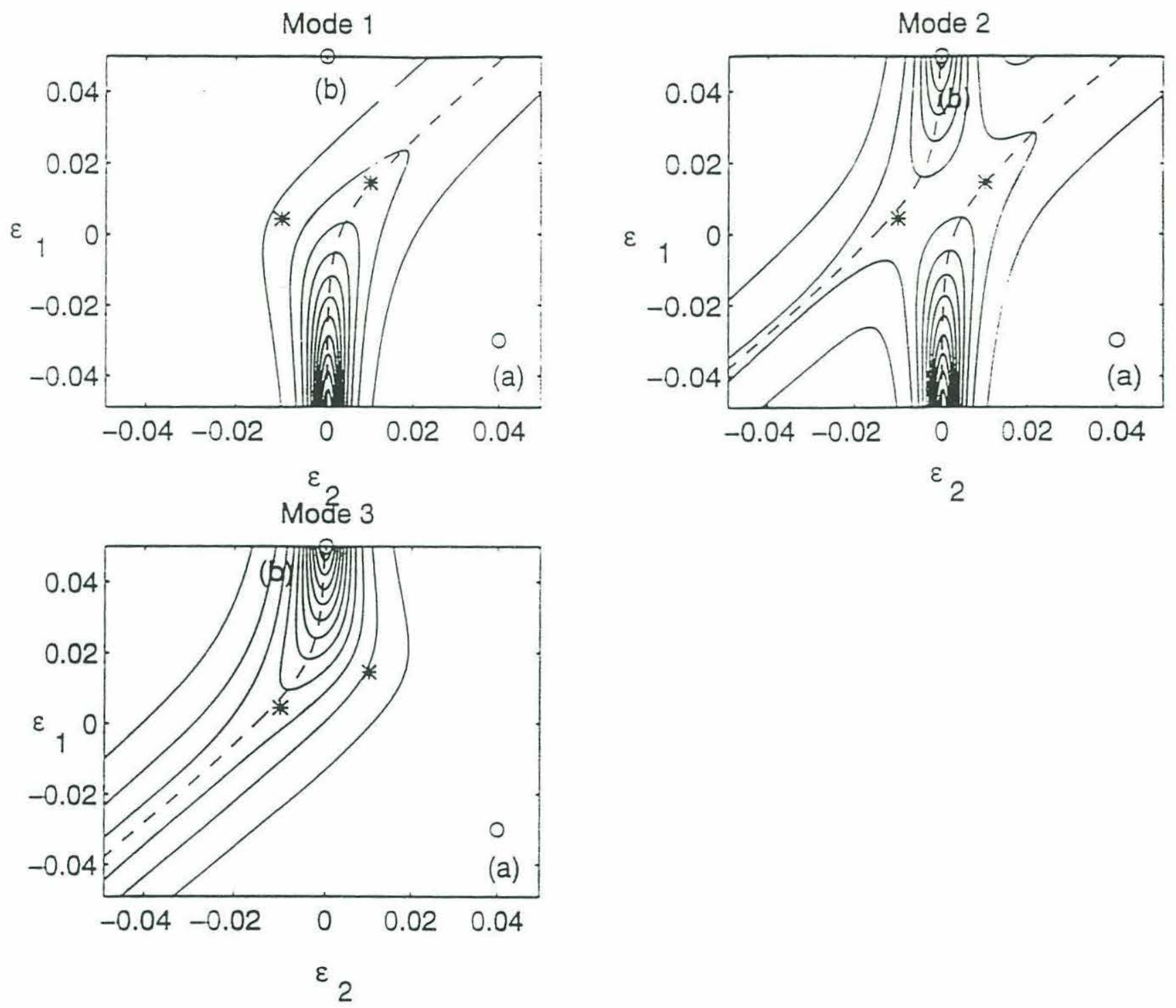

Figure 3-4: Modal Sensitivity $Q\left(q_{i}, \epsilon_{2}\right)$ for three pendula. 
cube-root strength branch points are marked on the same figure. They are located close to the origin. The zone close to the cube-root branch point is a zone where all three modes have significant sensitivity. We can see that the modal sensitivity contours vary in magnitude over different regions of the graph. We have not marked any magnitudes to prevent the figures from being too crowded but it is sufficient to note that zones where there is significant clustering of the sensitivity contours are also zones where there is increased sensitivity while zones where the contours are well spaced are also zones where there is reduced sensitivity. This variation is because the imaginary part of the branch point varies in magnitude over different zones of the graph. In zones where the imaginary part is large, we have low sensitivity while in zones where the imaginary part is small, we have large sensitivity. Similar figures can be obtained for $Q\left(q_{i}, \epsilon_{1}\right)$ and are plotted in Appendix C.

The cube-root strength branch point occurs close to where the square-root strength branch point surfaces converge. In the case of the branch point lines drawn in figure $3-4$, if we had permitted $\epsilon_{1}$ to be complex, we would have seen actual intersection of the square-root branch surfaces for some special value of $\epsilon_{1}$. The next question to be answered is which branch point is more important, the cube-root variety or the square-root variety, when both are close to each other ? In chapter 2, we have already seen that the square-root branch point is associated with two $\epsilon^{\frac{1}{2}}$ splits in the eigenvector perturbation. Since the modal sensitivity is directly related to the square-root term, it is reasonable to conclude that only two modes show appreciable sensitivity. Eigenvector perturbation expansions about the cube-root branch point have three modes which possess the $\epsilon^{\frac{1}{3}}$ dependence on disorder and hence in zones where the cube-root branch point effects are important, we would see three modes possessing large modal sensitivity. In the figure $3-4$, we can see that close to the origin all three modes possess appreciable sensitivity. Hence it is unlikely the square-root branch point is important in its effects close to the origin since we should expect to see significant modal sensitivity for only two modes.

We confirm our suspicions by using Jordan block expansion predictions at three points $P 1, P 2$ and $P 3$ which lie on the line $\epsilon_{1}=0$ and represent points with grad- 
(a) Good cube root expansion and bad square root expansion.
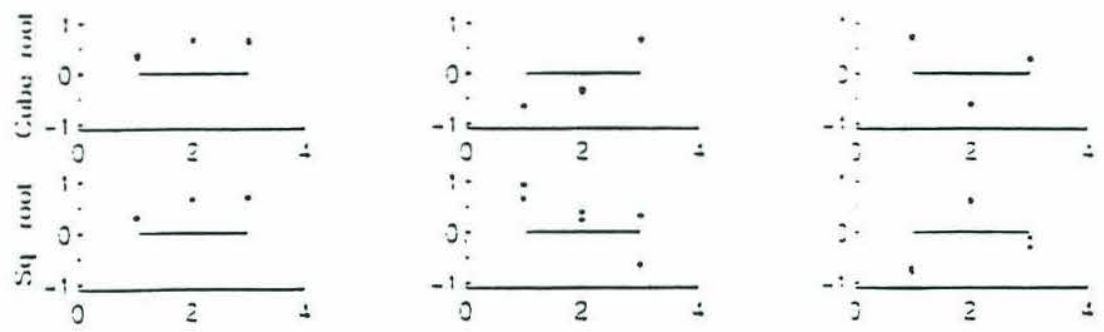

(b) Transition zone between areas where cube-root begins to perform badly and square-root branch point expansions begins to perform well.
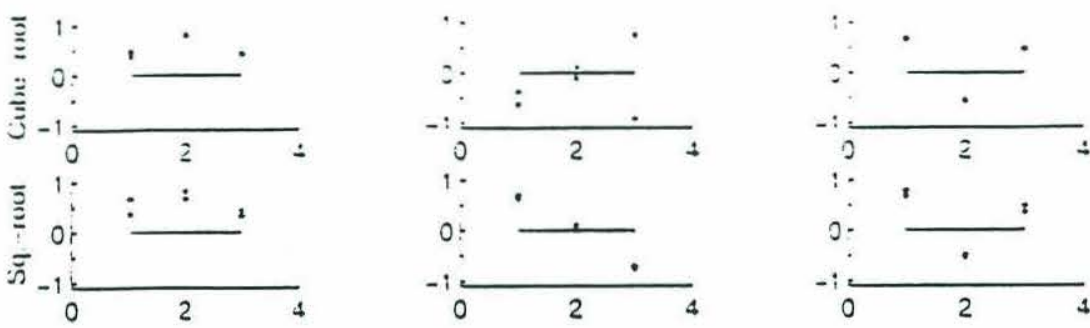

(c) Bad cube root expansion and good square root expansion.
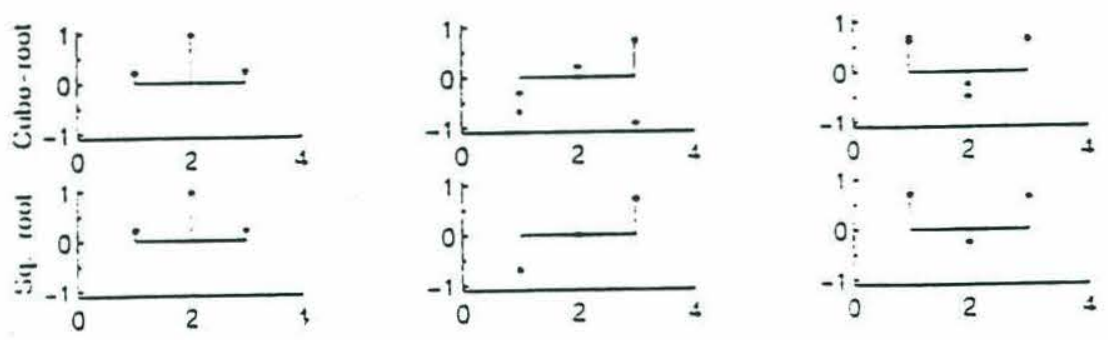

Figure 3-5: Gradual transition from cube-root branch point to square root branch point. Point P1 : $\epsilon_{1}=.0043, \epsilon_{2}=-.01186$. (a) : Jordan block size three expansion about (a). Jordan block size two expansion about $\epsilon_{1}=.00 \pm 3, \epsilon_{2}=$ $-.01186 \div .01616 i$. Point P2 : $\epsilon_{1}=.02, \epsilon_{2}=-.0033355$ i6. (b) : Jordan block size three expansion about (a). Jordan block size two expansion about $\epsilon_{1}=.02, \epsilon_{2}=$ $-.003335516+.0112921 i$. Point P3 : $\epsilon_{1}=.06, \epsilon_{2}=-.00018283$. (c) : Jordan block size three expansion about (a). Jordan block size two expansion about $\epsilon_{1}=.06, \epsilon_{2}=$

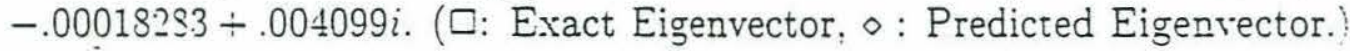




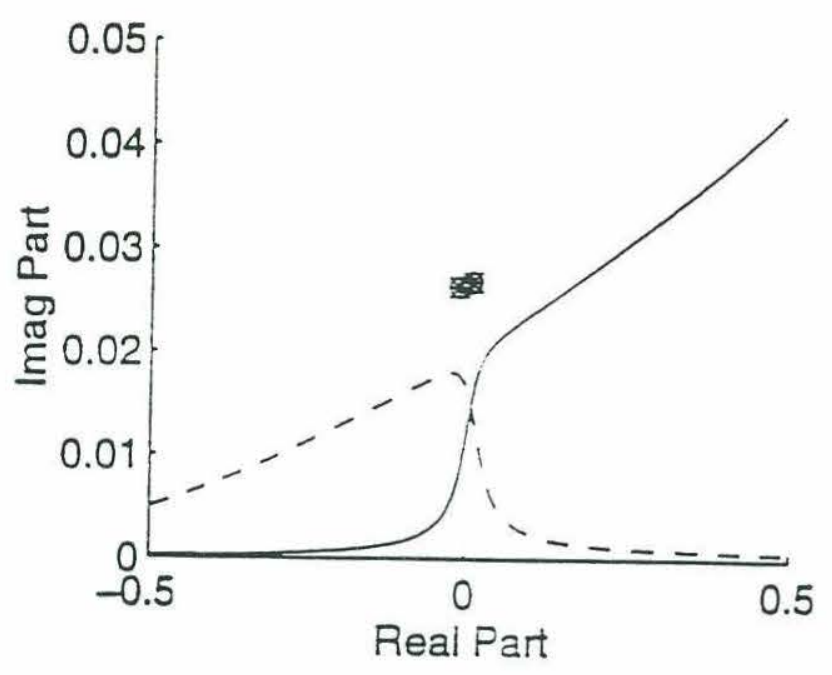

Figure 3-6: Variation of magnitude of $\operatorname{Imag}\left(\epsilon_{1}\right)$ with $\epsilon_{2}$ for square-root branch point curve. ०: Imaginary part of projection of cube-root branch point

ually increasing distances from the origin. These are shown in figure 3-5. Point $P 1$ $\left(\epsilon_{1}=.0043, \epsilon_{2}=-.01186\right)$ is a point where the Jordan block size three expansion about branch point (a) (3-5 a) is effective while the Jordan block size two expansion about $\left(\epsilon_{1}=.0043, \epsilon_{2}=-.01186+.01616 i\right)$ is ineffective. The Jordan block size two expansion blows up because of the close proximity of the higher order branch point. The point $P 2\left(\epsilon_{1}=.02, \epsilon_{2}=-.003335576\right)$ is a point in between where the Jordan block size three expansion (fig. 3-5 b) is beginning to function poorly while the size two expansion about $\left(\epsilon_{1}=.02, \epsilon_{2}=-.003335576+.0112921 i\right)$ is performing adequately. The point $3\left(\epsilon_{1}=.06, \epsilon_{2}=-.00018283\right)$ is a point where the Jordan block size 3 expansion (fig. 3-5 c) is inadequate while the Jordan block size two expansion about $\left(\epsilon_{1}=.06, \epsilon_{2}=-.00018283+.004099 i\right)$ performs well indicating that only the effects of the square-root branch point is important in this zone of the parameter space. So the broad conclusion is that in a zone of the parameter space where two different order branch points are present, the higher order branch point predominates in its effects.

Some more interesting features of the branch point geometry close to the cube-root 
branch point surface becomes obvious if we examine the imaginary parts associated with the square-root and cube-root branch points. We plot imaginary part of the square root branch point curve involving complex $\epsilon_{1}$ as a function of the real coordinate $\epsilon_{2}$ in figure 3-6. We superpose the projections of the positions of the three root coalescence on this plot. Clearly the large changes in the magnitude of the imaginary part occur close to the three root coalescence. Also the three root coalescence has a larger magnitude of the imaginary part than the square-root branch point. In the vicinity of the cube-root branch point the imaginary part of the square root branch point actually increases in magnitude while always being less in magnitude than that of the cube-root branch point. However, the effects of the cube-root would predominate close to the cube-root because a cube-root dependence is far stronger than a square-root dependence.

Let us say that we are examining a square root branch point with complex $\epsilon_{2}$. In figure $3-4$, we only considered real $\epsilon_{1}$ while evaluating the square-root branch point lines, but this was not necessary, and $\epsilon_{1}$ could be a complex but free parameter which we may vary. We now have the three eigenvalues with two eigenvalues being coincident along this surface. The box indicates the coincident eigenvalues.

$$
\left(\lambda_{1}, \lambda_{2}, \lambda_{3}\right)
$$

Let us say we have another square-root branch point line with complex $\epsilon_{1}$ and $\lambda$ being the unknowns obtained from solving equations 3-1 and 3-2. Two eigenvalues are coincident again but not the same two that we saw coincident earlier in figure 3-4.

$$
\left(\lambda_{1}, \lambda_{2}, \lambda_{3}\right)
$$

There is only one situation in which we could obtain a three root coalescence. That would occur if these two square-root branch point surfaces cross and the eigenvalues would now be of the form shown below :

$$
\lambda_{1}, \lambda_{2}, \lambda_{3}
$$


Schemaric I wo Parameter Geometry

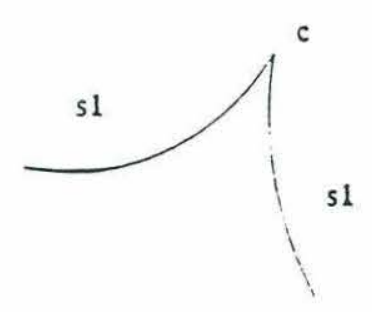

$$
\begin{aligned}
& \text { s1: Two root coajescence line } \\
& \text { s2 : Two root coalescence line } \\
& \text { c: Three root coarescence point }
\end{aligned}
$$

Figure 3-7: Schematic diagram of branch point surfaces for two parameter system

The obvious implication is that the two root coalescence is a subset of the three root coalescence since a two root coalesence is a necessary condition for a two root coaiescence to occur. We have noted that a square-root branch point can only be determined by solving two equations for eigenvalues coalescence. One of the disorder parameters can be varied arbitrariiy over the entire field of complex numbers to generate a two dimensional surfaceithe real and imaginary parts can be taken as independent coordinates). This surface is said to have codimension two since we need two equations to define the surface. We have two two-dimensional square-root branch point type surfaces crossing to generate a three root coalescence point. If twe restrict ourseives so that one of the parameter values is real and the other complex. we obtain loci for the branch points which approach each other but do not cross as in îgure 3-4. A schematic diagram of the distribution of these branch points is provided in figure 3- - for a two parameter system similar to the type we saw for three pendula.

We now examine the square-root branch point lines for the three penduia system. We have already noted that there are three cube-root branch points (ai. (b), and (c): Instead of permitting $\epsilon_{1}$ to be pure real, we specify that $\epsilon_{1}$ assume the same 
(a)

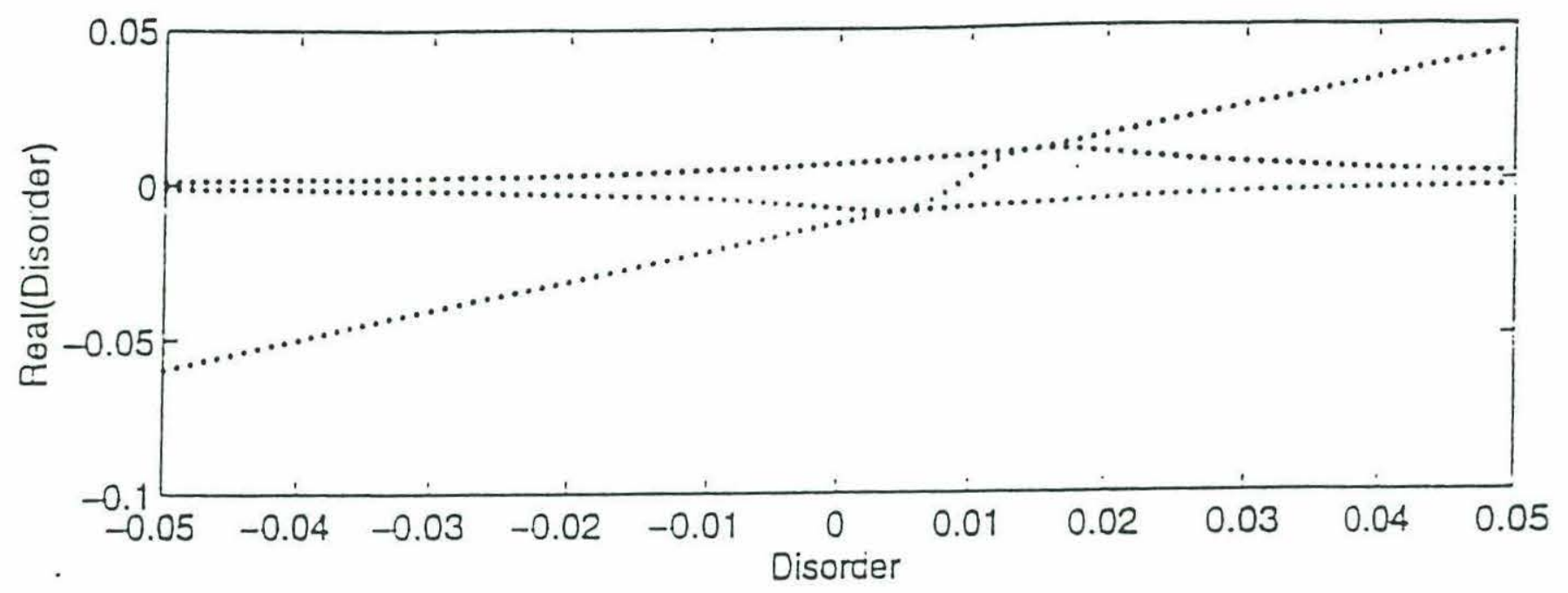

(b)

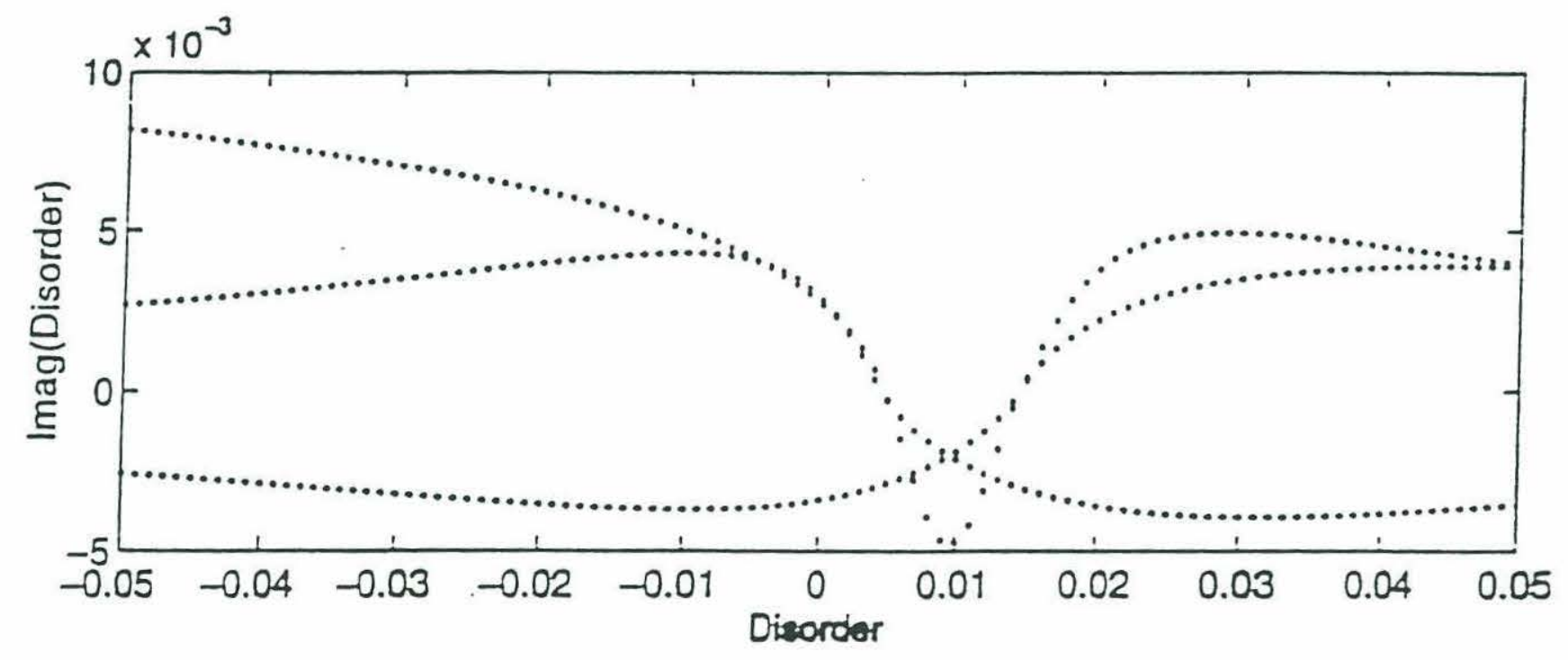

Figure 3-8: (a) : Real $\left(\epsilon_{2}\right)$ versus Imaginary $\left(\epsilon_{1}\right)$. (b) $: \operatorname{Imag}\left(\epsilon_{2}\right)$ versus $\operatorname{Imaginary}\left(\epsilon_{1}\right)$ 


\begin{tabular}{||c|c|c||}
\hline \hline Branch Point & $\epsilon_{1}$ & $\epsilon_{2}$ \\
\hline \hline (a) & $.0145 \pm .0268 i$ & .0101 \\
(b) & $.0043 \pm .0262 i$ & -.0099 \\
(c) & $.0099 \pm .0144 i$ & $-.0008+.0283 i$ \\
\hline \hline
\end{tabular}

Table 3.1: Three Root Coalescences

values as in figure 3-4 but with an imaginary part equal to that of cube-root branch point (a) ie $.0268 i$. We are thus taking a section in the complex space through the cube-root branch point instead of restricting ourselves to real values of $\epsilon_{1}$. We plot the real and imaginary parts of the complex coordinate $\epsilon_{2}$ obtained by solving equations 3-1 and 3-2 with $\mathrm{m}=1$, as a function of the real part of $\epsilon_{1}$ in figures 3-8a and $3-8 \mathrm{~b}$. The various branch point lines can only cross at a point of three root coalescence. As described earlier, for a system of eigenvalues $\left(\lambda_{1}, \lambda_{2}, \lambda_{3}\right)$, one of the branch point lines corresponds to a case where two eigenvalues say $\lambda_{1}$ and $\lambda_{2}$ are equal while the the second branch point line corresponds to a case where the other two eigenvalues, say $\lambda_{2}$ and $\lambda_{3}$ are equal. A cube root branch exists if both of the real and imaginary parts of the various branch point lines cross each other. If only one or the other cross each other, we do not have a cube-root branch point and in fact nothing special can be attached to this phenomenon. We can clearly see there are only two coordinates at which this occurs. Cube-root branch points occur if $\operatorname{Real}\left(\epsilon_{2}\right) \simeq .01$ and $\operatorname{Real}\left(\epsilon_{2}\right) \simeq-.01$. The imaginary part at the point of cross-over is zero for both branch points. Comparison with branch points which were calculated in Chapter 2 (and are presented again for ready reference in table 3-1) show that the the cube-root coalescence coordinates are indeed correct.

We will see these features for larger systems which are dependent on many parameters $\left(\epsilon_{1}, \ldots, \epsilon_{n-1}\right)$. We have a hierarchy of surfaces. The higher order branch point surfaces are embedded in the lower order branch point surfaces. The square root type branch point surface can be obtained by solving two equations and by systematically 
varying $(n-2)$ parameters to generate a surface of dimension $2(n-1)$. We could select these surfaces in $\frac{n !}{2 !(n-2) !}$ ways. Cube root branch point surfaces are generated wherever these square-root branch point surfaces intersect. We need three equations to determine these cube-root branch points. We can vary $(n-3)$ parameters at a time and hence the cube-root branch point surface is of dimension $2(n-3)$. In general, ${ }^{n} C_{m}$ $m$ th root branch point surfaces can be generated and these are of dimension $2(m-1)$ and require $m$ equations to be solved for the complex branch point coordinates.

We next consider the modulus of the modal sensitivity given by

$$
Q\left(q_{i}\right)=\sqrt{Q\left(q_{i}, \epsilon_{1}\right)^{2}+Q\left(q_{i}, \epsilon_{2}\right)^{2}}
$$

in figure 3-9(Modes 1-3). Here the contributions of all the partial modal derivatives have been accounted for and we can expect these contours to be similar to the localization factor contours because they reflect the integral of the modal sensitivity. We next plot the localization factor as a function of disorder over the same numerical range in figure $3-10$ (Modes 1-3). There is a remarkable similarity between the contour lines for modulus of modal sensitivity(3-9 Modes 1-3) and localization factor(3-10 Modes 1-3). The asymmetry in the localization factor curves appear to be directly related to the asymmetric position of the three root coalescence points, the projections of which have been marked on the graph.

We examine the mode shapes at points $a$ and $b$ on figure $3-4 b$. At (b), we are close to a two root coalescence curve and two modes have appreciable amplitudes. At (a), we are far from all branch points and all modes are heavily localized. These three modes are shown in figures 3-11 (a-c) where we show Jordan block size two expansion predictions and in figure 3-11 (d-f) where we show MPM predictions at point (a). Clearly the MPM performs better because of the point being distant from all branch points while the Jordan block size two expansion prediction is poor. In figure 3-11 (g-i), we show predictions using the Jordan block expansion for point (b). Clearly the Jordan block expansion does provide good predictions because of the proximity to the square-root branch point. The MPM blows up. The important feature here is that (a) 

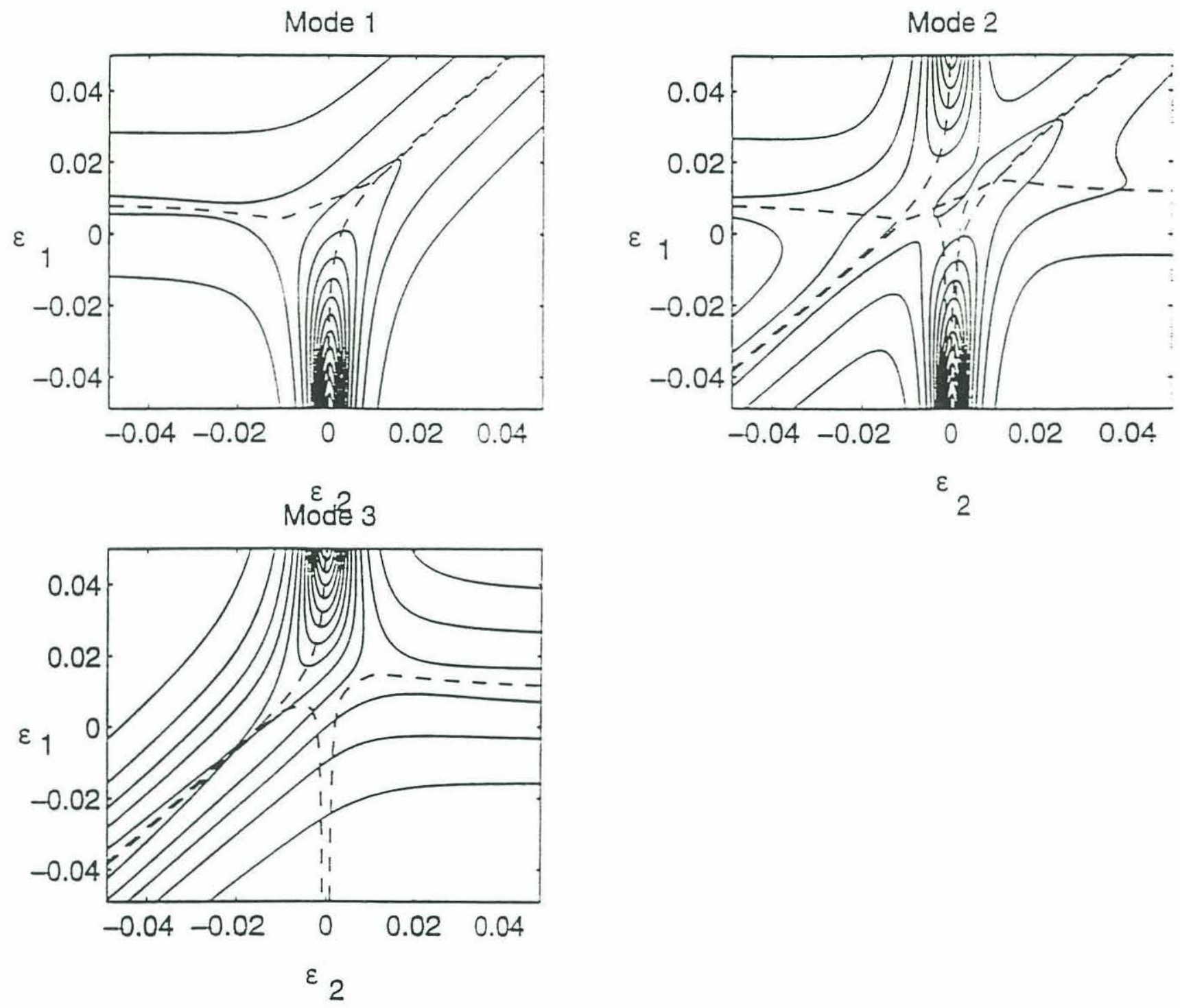

Figure 3-9: Modulus of modal sensitivity : $Q\left(q_{i}\right), i=1,2,3$ 



Figure 3-10: Localization factor contours for three pendula. $\star$ : Projection of cuberoot branch points on real disorder plane. 

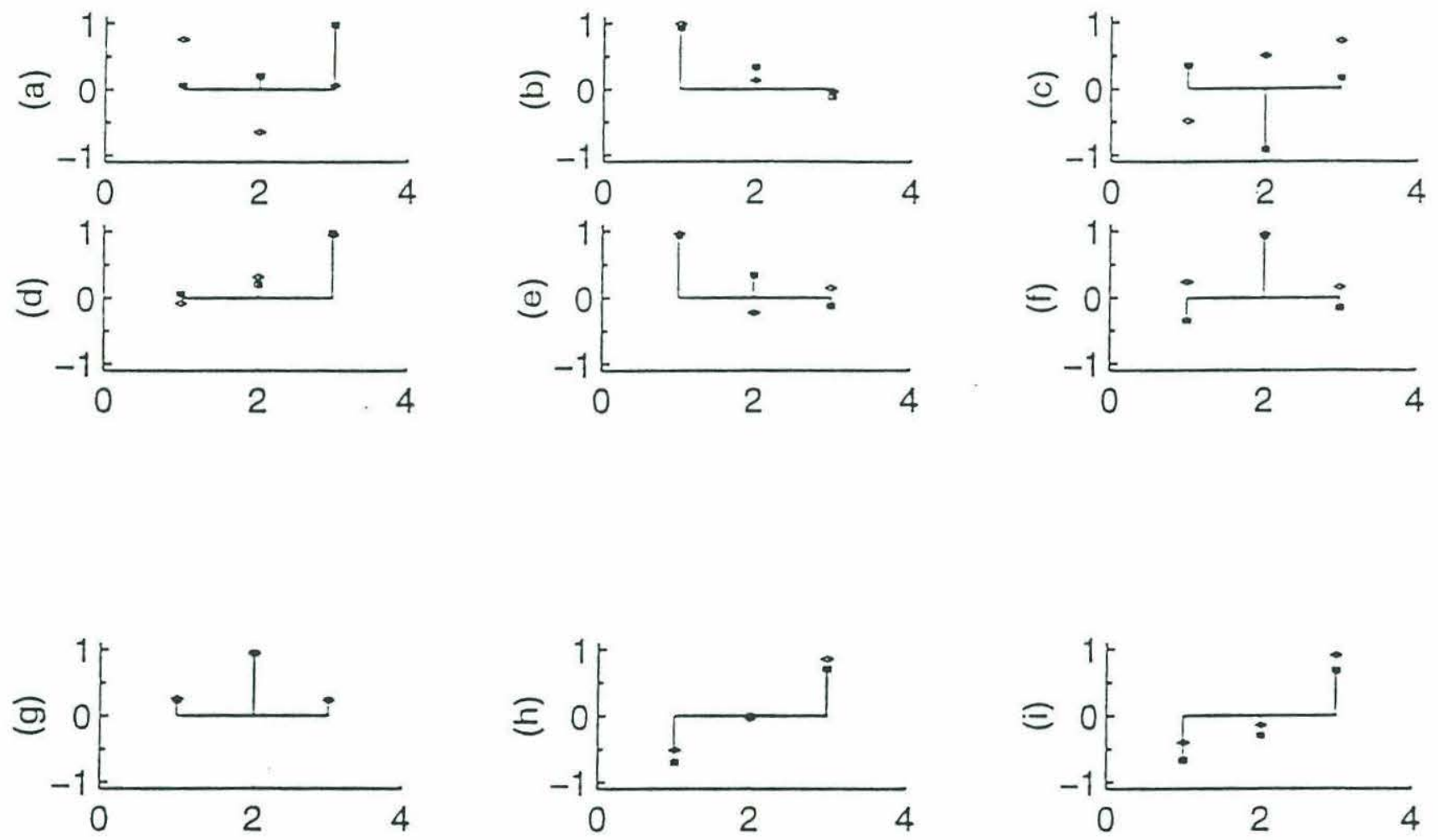

Figure 3-11: Figures (a-c): Mode Shapes at (a), $\epsilon_{1}=-.04, \epsilon_{2}=-.03$. a-c : Jordan block size two expansion about $\epsilon_{1}=-.03, \epsilon_{2}=-.042+.018 i$, d-f : .MPM expansion. Figures (d-f): Mode Shapes at (b), $\epsilon_{1}=0.05, \epsilon_{2}=0$. Jordan block size two expansion about $-0.0003+0.00$.ji . 
and (b) are disorder configurations possessing the same mean square disorder. Inspite of that, we see that (b) corresponds to nonlocalized modes whereas (a) corresponds to localized modes. This provides us with a good explanation of Hodges and Woodhouses discovery that statistically similar combinations of disorder yield significantly different levels of localization. The answer is that rather than any absolute magnitudes of disorder, the distance from that point in the parameter space to the closest branch point surface is the crucial factor in deciding the amount of localization seen in the modes. We have looked at a simple example here to explain this phenomenon but it is true for arbitrarily sized systems. Of course, we would have to determine the closest eigenvalue coalescences of all orders to find out the number of oscillators which have significant amplitude and the number of modes which are not localized.

It is clear there are regions of increased localization on these maps of the parameter space. In figure 3-12 a-r, we provide plots of variation of eigenvectors along the two square-root branches in figure 3-4 (a-c). The branch point lines are lines along which the modes display large sensitivity but have not undergone sufficient change to look localized. Evidently, the branch point lines are lines where it is sub-optimal to search for localization given that all the modes in figure 3-12 a-i look reasonably extended. We see in figures 3-12a through 3-12c that mode 1(figure 3-12a) is localized and mode 2 and 3 (figures 3-12b through 3-12c) have appreciable amplitude on two oscillators. In figures 3-12d through 3-12f, we are actually in a zone where the effects of three root coalescence are felt and although we have used coordinates for a two root coalescence, we are in reality seeing the effects of the three root coalecence. Further down the branch in figures 3-12g through 3-12i, we see that the modes have undergone appreciable change in relation to the modes in figure 3-12a through 3-12c. Now, the modes 2 and 3 (figures $3-12 \mathrm{~h}$ and 3-12i) are extended with appreciable amplitude on two oscillators while mode 1 (figure $3-12 \mathrm{~g}$ ) is localized. For much of the distance along this line there was relatively little change in the mode but owing to the three root coalescence, there was a violent change in the mode whence the mode remained relatively unchanged for much of the second half of the branch point line. The main difference between the modes in figures $3-12$ a through $3-12 c$ and those 
(a) : Modes along branch B1
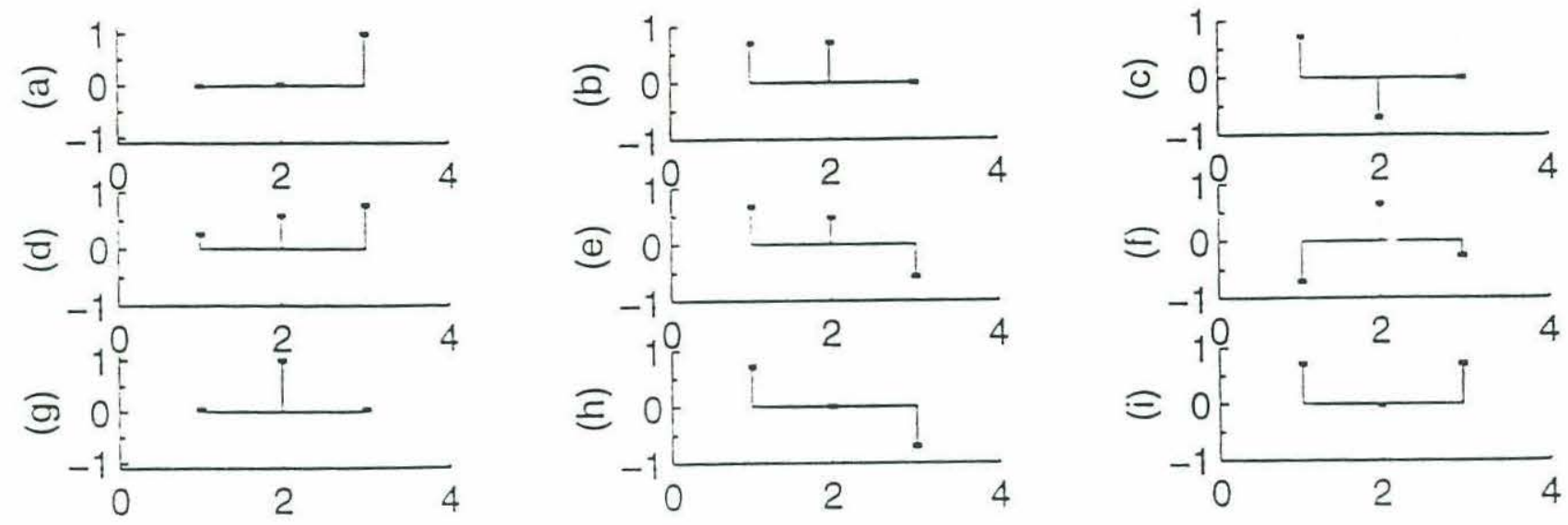

(b) : Modes along branch B2


Figure 3-12: Mode Shapes (a-c): $\epsilon_{1}=-.48, \epsilon_{2}=-.428$, Mode Shapes(d-f) : $\epsilon_{1}=0$, $\epsilon_{2}=-.0152$, Mode Shapes (g-i): $\epsilon_{1}=0, \epsilon_{2}=.49$, Mode Shapes $(\mathrm{j}-1): \epsilon_{1}=-.48$, $\epsilon_{2}=0$, Mode Shapes $(\mathrm{m}-\mathrm{o}): \epsilon_{1}=.003, \epsilon_{2}=0$ Mode Shapes $(\mathrm{p}-\mathrm{r}): \epsilon_{1}=.49$, $\epsilon_{2}=.4675$ 
in $3-12 \mathrm{~g}$ through $3-12 \mathrm{i}$ is that the cube-root branch point has caused the modes to switch the oscillators on which there were significant amplitudes. Thus modes in figures 3-12b and 3-12c had significant amplitudes on the first and second oscillators while modes in figures $3-12 \mathrm{~h}$ and $3-12 \mathrm{i}$ had significant amplitudes on the first and third oscillators. Modes a and $\mathrm{g}$ were localized but with the oscillator with large amplitude being the third and second respectively. There is an analogy existing with the curve veering (Perkins and Mote [25]) seen earlier in the manner of eigenvector exchange which occurs, although here the veering is that associated with the squareroot branch point curve. Modes 3-12j through 3-12r represent modes at points on B1 corresponding to those in figures $3-12$ a through $3-12 \mathrm{i}$ on the branch B2. Modes in figures $3-12$ a through $3-12 \mathrm{c}$ and those in figures 3-12p through $3-12 \mathrm{r}$ on the one hand and those in figures $3-12 \mathrm{~g}$ through $3-12 \mathrm{i}$ and in figures $3-12 \mathrm{j}$ through $3-12 \mathrm{l}$ on the other are the same but shuffled around indicating that interchange of mode shapes has occurred along these branch point loci. This feature explains another aspect of the asymmetry seen in figure 3-10 (Modes 1-3) for the localization factors for the three modes of the three pendula system. The localization factors of mode 1 form a triangle while those of mode 3 appear to also form a triangle but with the vertices of the triangle rotated around. The reason is that during the veering the modes associated with the branch point lines have been exchanged and the localization factor contours reflect this fact.

Please see Appendix C for square-root type branch point surfaces for a four pendula system.

\subsection{Optimal directions to maximize localization}

The asymptotes of the branch point surfaces appear to be straight lines. The bisectors between the asymptotes of the square-root type branch point surfaces appear to be zones of increased localization in figure 3-10(Modes 1-3). Any search to determine optimal combinations of disorder for localization must proceed in an initial direction along this bisector. Some bisectors appear to be more conducive to localization than 


\section{Mode 2}



Figure 3-13: Optimal Search Directions to maximize localization superposed on localization factor contours for mode 2: '-' : Directions al-a6.

others. It is not clear what precise criterion is to be used to determine which of these bisectors ensures more localization. The asymptotes are obtained by obtaining squareroot branch point solutions for equations 3-1 and 3-2 for the asymptotic ralues of the disorder i.e. $\epsilon_{1} \rightarrow \infty, \epsilon_{1} \rightarrow-1$ with complex $\epsilon_{2}$ and $\epsilon_{2} \rightarrow \infty, \epsilon_{2} \rightarrow-1$. with complex $\epsilon_{1}$. In figure $3-13$, we provide plots of the bisectors to the asymptotes superposed on the contour lines for mode 2 of the three pendula system. Since all the two root coalescences cause interaction of this mode with the other modes, this mode is the least localized of the three modes. It is fairly obvious that the localization is maximum along these bisectors and the localization is more in some of these directions than the others. For example, directions a 3 and a 6 offer more localization for the same mean square disorder than the other directions. There are no consistent conditions which can be used to determine which of these directions offer the most localization. But it is of interest that the optimal direction a6 actually lies along the direction away from the three root coalescence, whereas the direction a 3 lies in the direction of a cube-root branch point. Between a 3 and a6, a6 provides more localization for the same mean square disorder. 
These general trends of the optimal directions being along the bisector between adjacent square-root branch point surfaces is true for systems with more disorder parameters. However, instead of square-root branch point lines, we would be dealing with a branch point surface in the $n$ dimensional space. We would again have to determine the bisectors between adjacent square-root branch point surfaces to determine the optimal directions for maximizing localization.

\subsection{Quantifying difference between two and three root coalescences.}

The main difference between the two and three root coalescence so far has been in the number of modes showing sensitivity to applied perturbation. Another quantitative approach to show this difference is by using perturbation method predictions. In short if we are close to a cube root branch point, a cube-root perturbation expansion would provide good predictions while if we are close to a square-root branch point a square-root expansion would work well.

In figure 3-14, we show predictions of the eigenvalue loci as we traverse the contour $\epsilon_{1}=0$. As we start from $\epsilon_{2}=-.05$, we first encounter a square-root branch point and then two cube-root branch points and finally a square-root branch point before we approach $\epsilon_{2}=.05$. Jordan block size two predictions of the eigenvalue loci are shown in figure 3-14 a. In figure 3-14 b, we show Jordan block size three predictions of the eigenvalue loci about branch point (b). In figure 3-14c we show Jordan block size three prediction of the eigenvalue loci for the expansion about branch point (a). Finally we show the Jordan block two expansion prediction for eigenvalue loci close to the square-root branch point of coordinates. The expansions close to the square-root branch points appear to be associated with a veering of the eigenvalue loci as predicted

in Triantafyllou and Triantafyllou [39]. The intermediate cube-root branch points are very closely spaced hence each cube root expansions works well over a short range before failing. These also happen to be the zone closest to the origin. The square-root 
a

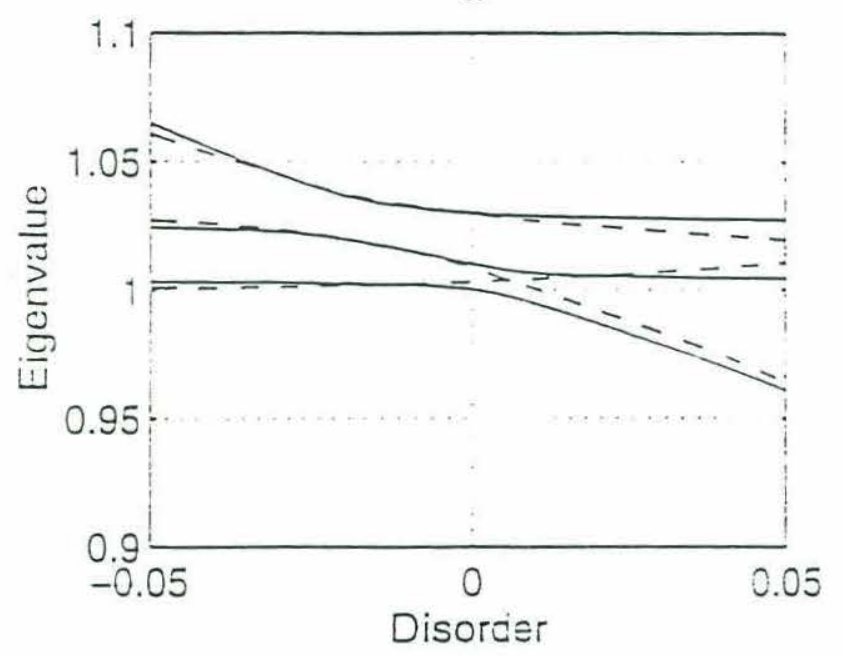

C

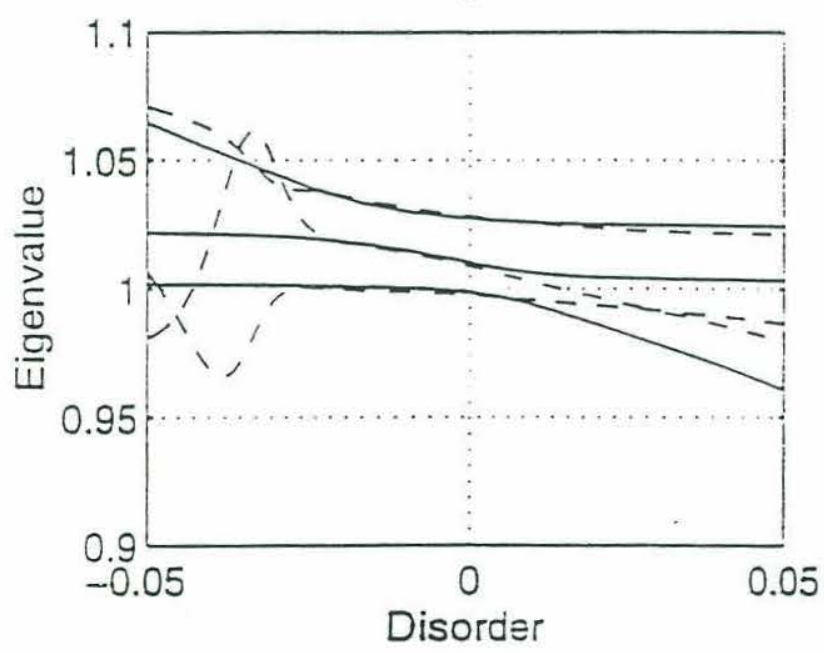

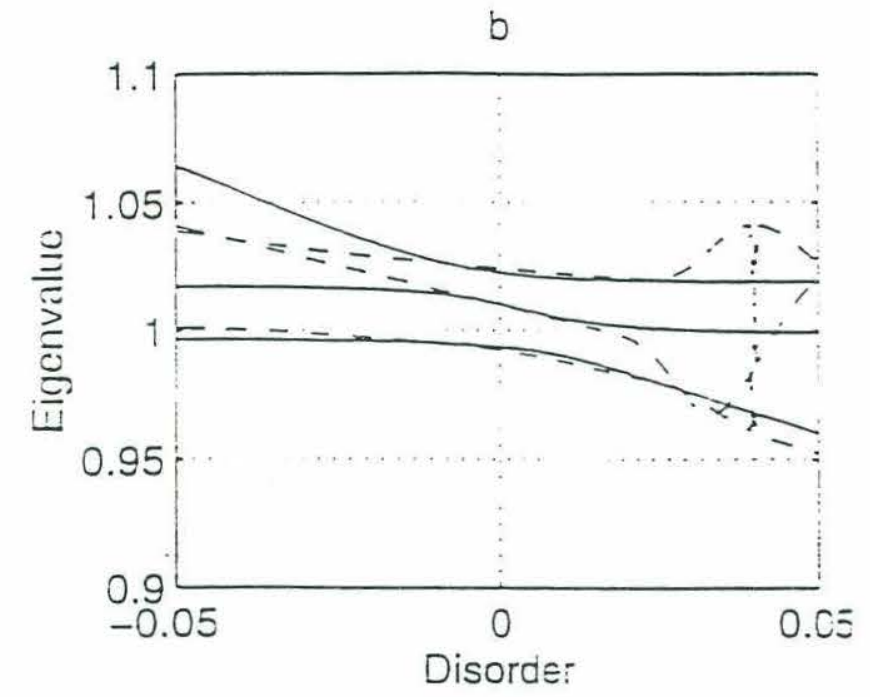

d



Figure 3-14: (a) : Jordan block size two expansion about $\epsilon_{1}=0, \epsilon_{2}=-.0152 \div .0169 i$. (b) : Jordan block size three expansion about branch point (a). (c) : Jordan block size three expansion about branch point (b). (d) : Jordan block size two expansion about $\epsilon_{1}=0, \epsilon_{2}=.0030-.0113 i$ 


\begin{tabular}{||c|c|c|c|c|}
\hline \hline$\epsilon_{1}$ & $\epsilon_{2}$ & $\epsilon_{3}$ & $\epsilon_{4}$ & $\epsilon_{5}$ \\
\hline \hline$-.0218-.0781 \mathrm{i}$ & $-.0941-.0816 \mathrm{i}$ & $-.1126+.0339 \mathrm{i}$ & $-.0483+.0555 \mathrm{i}$ & $-.1397-.02731 \mathrm{i}$ \\
\hline \hline
\end{tabular}

Table 3.2: Six Root Coalescences

branch points influence sensitivity for larger disorder. This confirms the general trend we observed that the strongest ( $n$th root) branch point occurs closest to the origin. The lower order branch points can occur for larger values of disorder and can also possess smaller imaginary parts. Depending on the trade-off between the magnitude of the imaginary part and the strength, we could get lesser or greater sensitivity for the lower order branch point relative to the higher order branch points.

\subsection{Conflicting effects of strength and distance from the real axis : Trends and Examples}

We illustrate this point with a very interesting example i.e. a system of six coupled pendula. We consider an extreme case where the disorder is of the order of $50 \%$ of the length to highlight the problem in a clearer fashion. Consider two possible cases viz. the first being one where we have the effects of a six root coalescence being important and the second where we have the effects of two three root coalescences being important. The complex coordinates of one of the complex branch point coordinates is shown in table $3-2$.

We show the modes of the six pendula system with the six root coalescence being important. The modes are those corresponding to that of the periodic state. These are shown in figures 3-15 a-f. The case corresponding to the system where the effects of two three root coalescences are important is next discussed. Such a system is seen in a system of six pendula with three of them having a length of two units and the other three having a length of one unit. The disorder combination for the special 




(a)

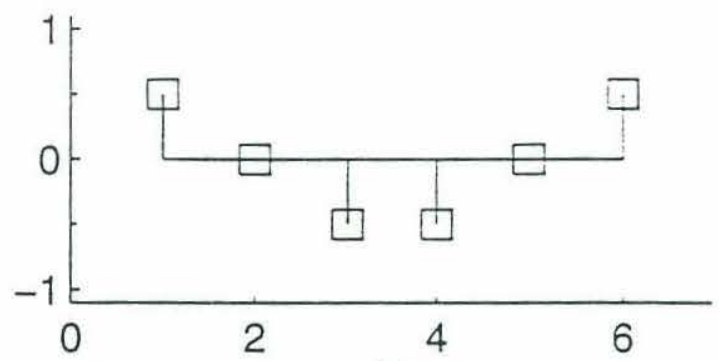

(c)

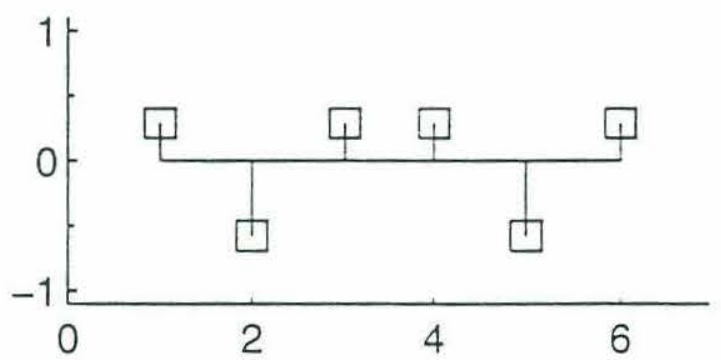

(e)

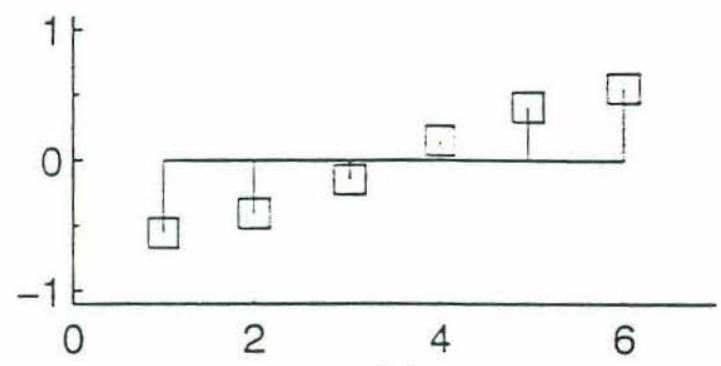

(b)

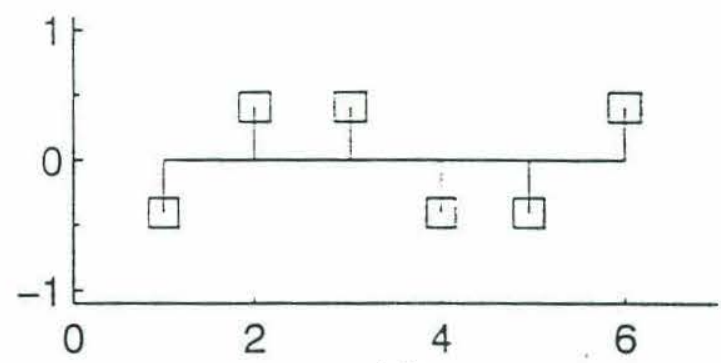

(d)

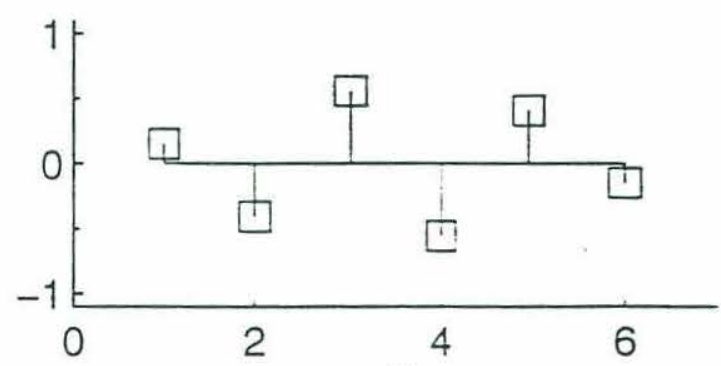

(f)

Figure 3-15: Six Pendula System : Modes close to Six Root Coalescence 


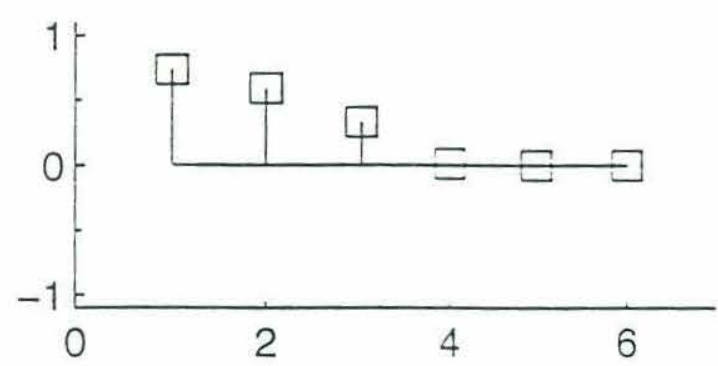

(a)

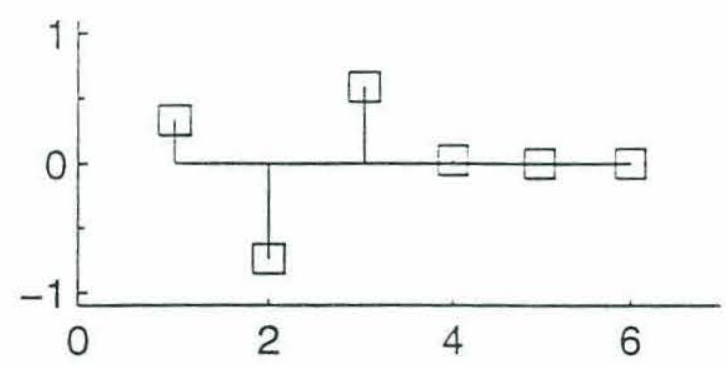

(c)

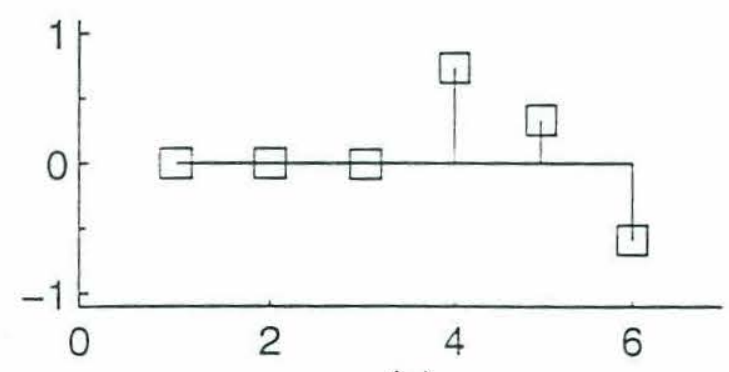

(e)



(b)

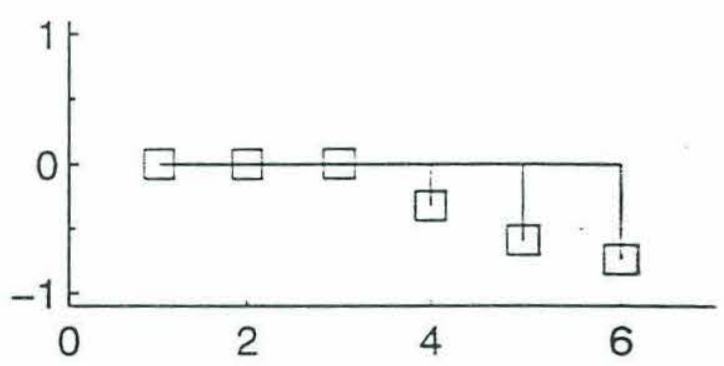

(d)

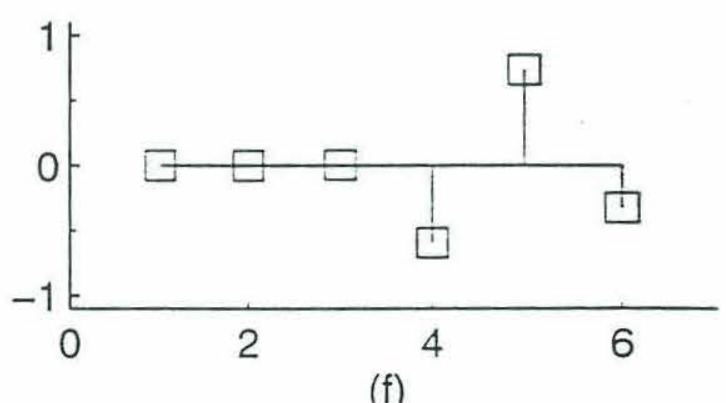

Figure 3-16: Six Pendula System : Modes close to Three Root Coalescences 


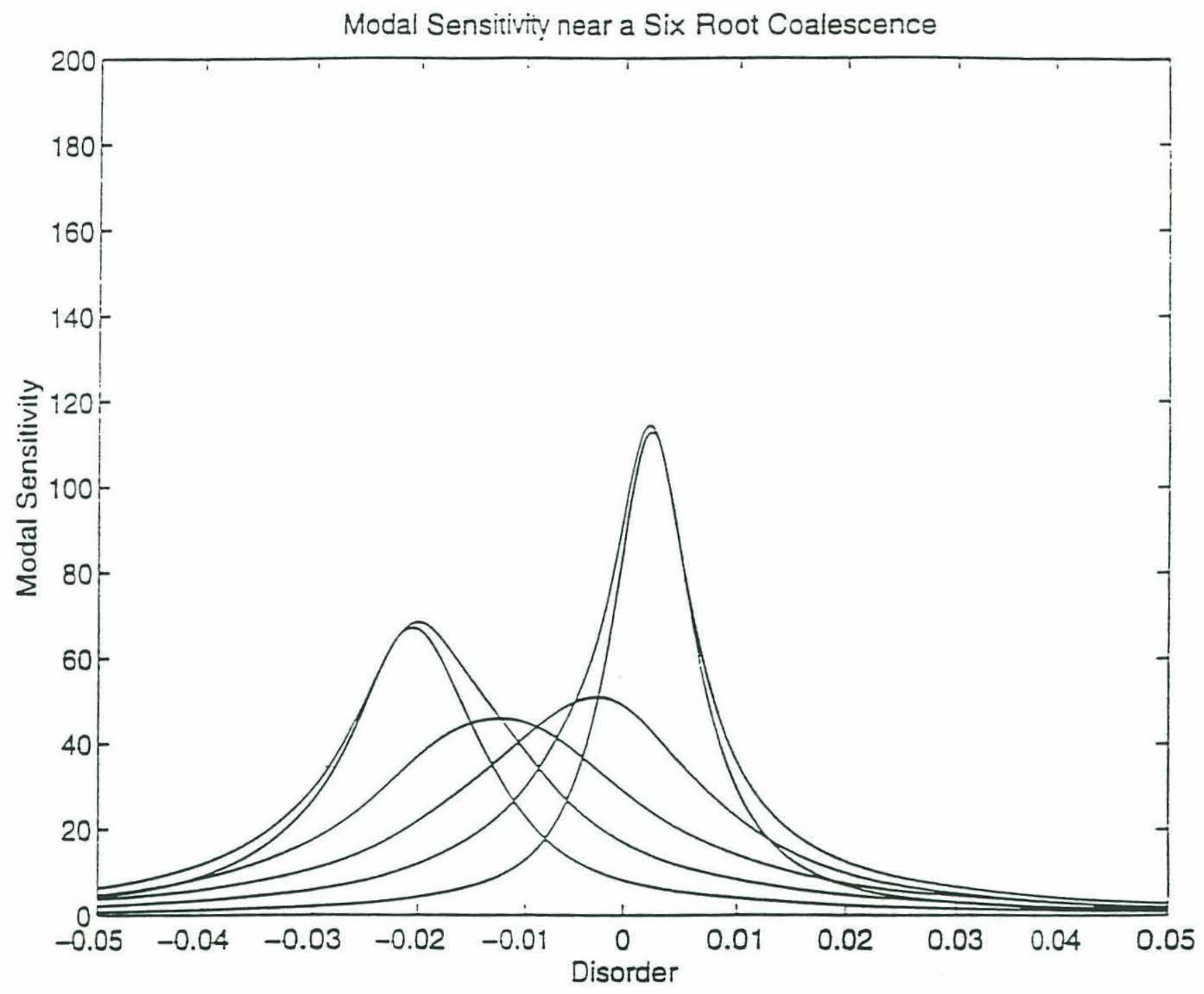

Figure 3-17: Six Pendula System : Modal Sensitivity for six root coalescence : $Q\left(q_{i}, \epsilon_{5}\right)$ where $1 \leq i \leq n$. 


\begin{tabular}{||c|c|c|c|c|}
\hline \hline$\epsilon_{1}$ & $\epsilon_{2}$ & $\epsilon_{3}$ & $\epsilon_{4}$ & $\epsilon_{5}$ \\
\hline \hline 0 & 0 & 0 & $1.0155-.1058 \mathrm{i}$ & $1.000796+.00000693 \mathrm{i}$ \\
\hline \hline
\end{tabular}

Table 3.3: Three Root Coalescence for configuration 1

configuration associated with two three root coalescences is as follows :

$$
\epsilon_{0}=\epsilon_{1}=\epsilon_{2}=1 . \epsilon_{3}=\epsilon_{4}=\epsilon_{5}=0
$$

. The modes are shown in figures 3-16 a-f. There are two sets of modes, group (a) and group (b). The former has significant amplitudes on the first three pendula while the latter has significant amplitude on the last three pendula. We plot the modal sensitivity as a function of $\epsilon_{5}$ for the six root coalescence problem in figure 3-17.

We consider the modal sensitivity for the three root coalescence as a function of $\epsilon_{0}$ for the group (a) modes and as a function of $\epsilon_{5}$ for the group (b) modes. These are plotted in figure 3-16. Note the peak sensitivity of the group (a) set of modes is much lower than that for the six root coalescence. The peak sensitivity for the group (b) set of modes is higher than that for the group (a) set of modes but less than the six root coalescence. Note also the group (a) set of modes appear to be completely decoupled from the group (b) set of modes. In other words, the group (a) set of modes are not sensitive to variations in $\epsilon_{5}$ and the group (b) set of modes are not sensitive to variations in $\epsilon_{0}$. We provide the complex coordinates of one of the branch points for this configuration in table 3-3. The magnitudes of the imaginary parts for the three root coalescences in table 3-3 are much smaller than those for the coordinates of the six root coalescence in table 3-2.

We now consider a second configuration where the first three modes have a length of .5 units while the last three pendula have a length of 1 unit. The modal sensitivities 
(a)

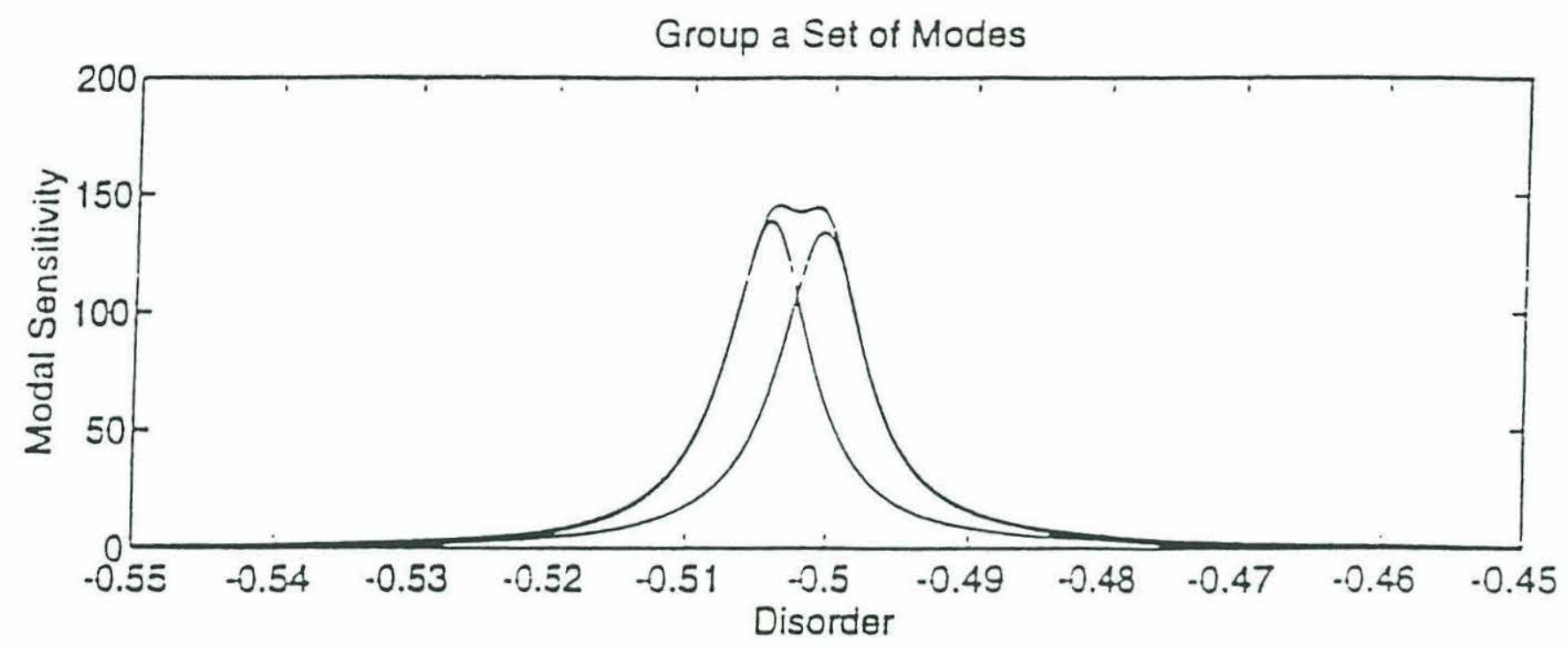

(b)

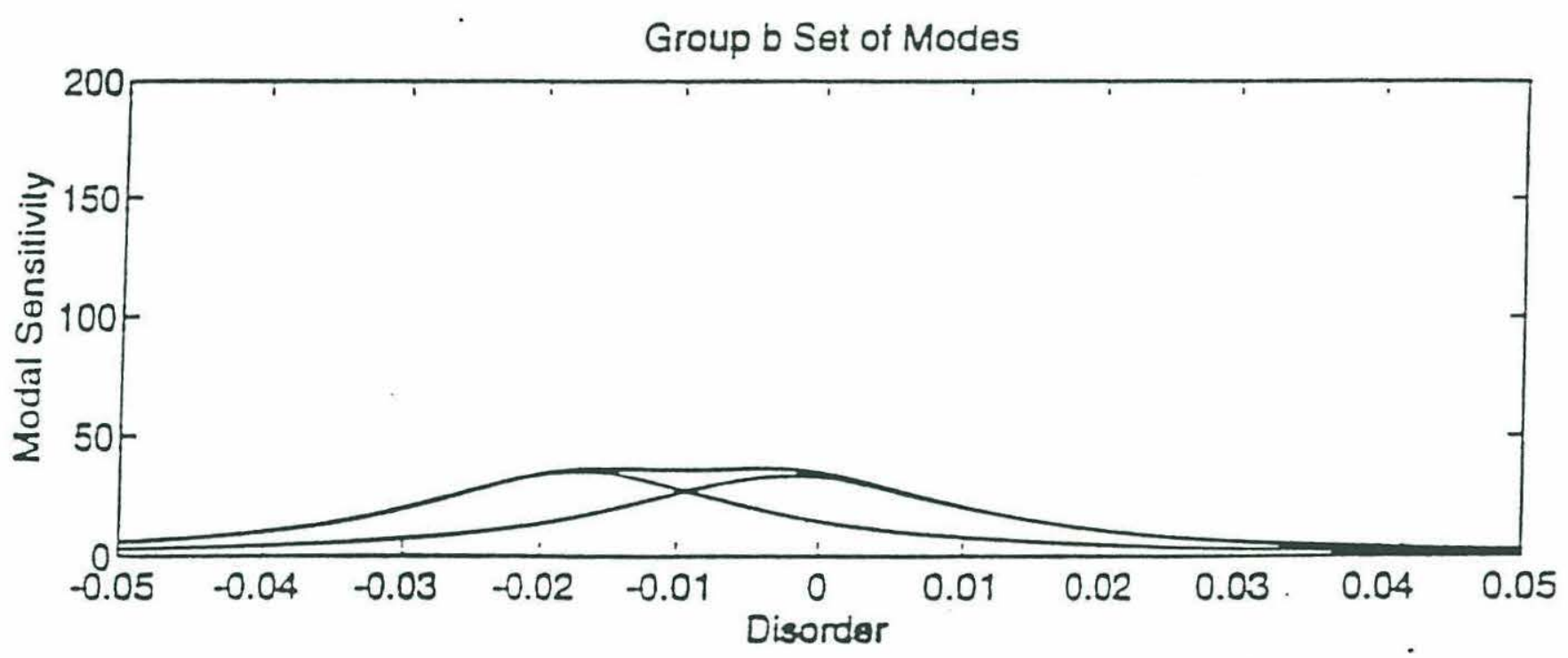

Figure 3-19: Modal Sensitivity for the three root coalescences. Group (a): $Q\left(q_{i}, \epsilon_{0}\right)$ and Group (b): $Q\left(q_{i}, \epsilon_{5}\right)$, where $1 \leq i \leq n$. 


\begin{tabular}{||c|c|c|c|c||}
\hline \hline$\epsilon_{1}$ & $\epsilon_{2}$ & $\epsilon_{3}$ & $\epsilon_{4}$ & $\epsilon_{5}$ \\
\hline \hline 0 & 0 & -.5 & $-.501143-.0065258 \mathrm{i}$ & $-.50003+.000000108725 \mathrm{i}$ \\
\hline \hline
\end{tabular}

Table 3.4: Three Root Coalescence

are plotted as a function of $\epsilon_{0}$ and $\epsilon_{5}$ in figure 3-19(a-b). The configuration is as below

$$
\epsilon_{0}=\epsilon_{1}=\epsilon_{2}=-.5, \epsilon_{3}=\epsilon_{4}=\epsilon_{5}=0
$$

. Only three modes display appreciable sensitivity. Previously, the group (a) set of modes displayed almost no sensitivity relative to the six root coalescence while the group (b) set of modes displayed lower but comparable sensitivity. Now the group (a) set of modes display slightly larger sensitivity than the six root coalescence while the group (b) set of modes display the same sensitivity as earlier. The obvious conclusion would be one of the three root coalescences has moved as we altered the length of the pendula. In table 3-3, we provide the complex coordinates of the three root coalescence branch point corresponding to the new configuration. The imaginary parts of the complex coordinates have clearly decreased.

In the first case(figure 3-19a) the three root coalescence was relatively far away from the real disorder axis and the modal sensitivity was very low. In the second case (figure 3-19b) the three root coalescence was relatively very close to the real disorder axis and the modal sensitivity was comparable with the six root coalescence even though the dependence on the disorder was a third root dependence as opposed to the sixth root dependence we saw earlier. Of course, the imaginary components of the six root coalescence were fairly large relative to the imaginary parts seen for the three root coalescences but the sixth root dependence on the disorder was responsible for the relatively large sensitivity. 


\subsection{Conclusions}

We provide numerical confirmation of the asymptotic results of Triantafyllou and Triantafyllou. Some broad trends in the numerical investigation of this problem were detected. The "strongest" branch point, corresponding to an $n$th root branch point for $n$ pendula occurred, typically, for values of small disorder and were fixed and relatively further away from the real axis. The weaker branch points corresponding to an $m$ th root (where $m<n$ ) dependence of modes on disorder were "movable" branch points and typically had larger imaginary parts as they approached the stronger branch point. For larger values of disorder the weaker ( $m$ th root) branch points actually had sufficiently small imaginary parts to result in modal sensitivities comparable to or greater than those for the stronger branch points, but would only affect $m$ modes. A form of curve veering was observed where the square-root branch point loci for a three pendula problem were seen to exchange loci as the branch point approached each other before veering away. The bisectors to adjacent square-root branch point surfaces were found to be lines along which localization was maximum. Among these directions, one of them actually was the most optimal direction to maximize localization but there does not appear to be any quantitative means to identify the most optimal direction. 


\section{Chapter 4}

\section{Optimal Mode Localization}

\subsection{Introduction}

Mode Localization offers very exciting possibilities for reducing vibration transmission in periodic structures like bridges, moorings, and offshore structures. The problem with these structures is that the excitation frequency which causes vibrations is not monochromatic. Conventional methods have focused on using anti-resonances to confine vibrations about the source. But if the exciting source has a frequency spectrum with the excitation frequencies spread over a broad range relative to the bandwidth of the resonances of the structure, we would be unable to achieve vibration confinement. The importance of mode localization is that even if we excite the structure at resonance, the vibration is still confined close to the source. There have been some situations where this method has been applied successfully. Cornwell and Bendiksen [9] has commented about the deliberate mistuning of blade assemblies to ensure some level of localization in turbines. However the disorder parameter selection was essentially a trial and error selection. There is a need to develop a systematic method for parameter selection while using localization in vibration isolation. This however required a mature understanding of modal sensitivity which was only acquired in the previous chapter.

Introducing disorder into an otherwise periodic structure results in manufacturing and aesthetic problems. Manufacturing problems are related to the fact that it is 
expensive to manufacture nonstandard parts. There is also a matter of aesthetic beauty in that public utilities like bridges would look ugly by having very irregular spans. We have already observed that there exists a mapping from the disorder parameter space to the eigenvalues determined by the solution to the characteristic polynomial. This mapping divides the parameter space into zones of higher and lower order localization. The transition from periodic to localized modes occurs very rapidly in a small range of parameters close to the solutions of the bifurcation equations. Hence we can accommodate our requirements of having as little disorder as possible while trying to maximize localization with this prior knowledge of the behavior of systems which exhibit localization.

\subsection{Work done in this chapter}

In this chapter, we study two optimization problems. The first problem, is that of parameter selection to ensure all the modes are localized to some minimum level while ensuring the sum of the squares of the disorder was a minimum. The second problem is that of maximizing localization while ensuring that the sum of the squares of the disorder is some specified amount. Numerical tests on small systems of pendula indicated that the distribution of optima is such that the optima are along lines of maximum distance from the lines of two root coalescence. Also, the convergence basins of each optimum are bounded by different lines of two root coalescence. An algorithm is suggested for tracking down all the optima with a view to determining the global optimum using this knowledge of the location of the optima. Since there was an exponential growth of optima and computational effort with the number of disorder parameters, a statistical analysis was performed to show that for small systems of pendula (two to six pendula), it was sufficient to sample only a few optima to obtain a good estimate of the global optimum. Special optimal solutions were also examined. 


\subsection{Statement of the Problem}

In this chapter, we will answer two fundamental questions. How do we select parameters (disorder) for the system so as to ensure a certain minimum level of localization? Alternately, if the constraint being placed on the system is that the disorder in the system has some mean value, the obvious question would be how do we ensure optimum selection of parameters to maximize the localization seen in the system?

\subsubsection{Problem 1 :Minimum Disorder to attain Minimum Level of Localization}

We use the same definition for localization factor used earlier. Consider a generic system of $n$ coupled pendula with $(n-1)$ possible disorder units $\epsilon_{i}$. We then state the optimization problem to ensure all the modes have a minimum localization factor. Minimize

$$
f\left(\epsilon_{i}\right)=\sum_{i=1}^{i=n-1}\left(\epsilon_{i}^{2}\right)
$$

subject to the constraints

$$
\gamma_{i} \geq b
$$

for $1 \leq i \leq n$, where $b$ is some minimum value of the localization factor as defined in appendix A.

\subsubsection{Problem 2: Maximize Localization for given Mean Disorder}

Maximize

$$
f\left(\epsilon_{i}\right)=\sum_{i=1}^{i=n} \frac{1}{\gamma_{i}}
$$

with the constraint 


$$
\sum_{i=1}^{i=n-1}\left(\epsilon_{i}^{2}\right)=n(b)^{2}
$$

Here $b$ is the mean desired disorder. If we need $5 \%$ disorder, we would $b=.05$.

\subsubsection{Problem 1: Objective Function}

We now state the optimization problem in terms of optimization theory.

$$
f\left(\epsilon_{i}, \lambda_{i}, c_{i}\right)=\sum_{i=1}^{i=n-1}\left(\epsilon_{i}^{2}\right)+\sum_{i=1}^{i=n} \lambda_{i}\left(\gamma_{i}-b-c_{i}^{2}\right)
$$

The objective function is used as defined earlier. The variables $\lambda_{i}$ are Lagrange Multipliers and the variables $c_{i}$ are slack variables used to implement the inequality constraints. Note the increase of the number of unknowns from $(n-1)$ variables $\epsilon_{i}$ to $3 n-1$ unknowns including $n$ additional unknowns in the Lagrange multipliers $\lambda_{i}$ and $n$ unknowns in the form of the slack variables $c_{i}$. The optimal solution exists as the solution to the system of equations

$$
\frac{\partial f}{\partial \epsilon_{i}}=\sum_{i=1}^{n-1}\left(2 \epsilon_{i}\right)+\sum_{j=1}^{j=n} \frac{\partial \gamma_{j}}{\partial \epsilon_{i}}=0
$$

$1 \leq i \leq n-1$

$$
\frac{\partial f}{\partial \lambda_{i}}=\gamma_{i}-b-c_{i}^{2}=0
$$

$n \leq i \leq 2 n-1$.

$$
\frac{\partial f}{\partial c_{i}}=c_{i} \lambda_{i}=0
$$

$2 n \leq i \leq 3 n-1$.

This is the complete statement of the problem. The equations are coupled, nonlinear algebraic equations, and we will have to use some iterative method to obtain the solution. Note there is a total of $3 n-1$ equations in $3 n-1$ unknowns.

Note the following feature about the equations. The third set of equations. involves the product of the slack variable and Lagrange multiplier. The Lagrange 
multiplier is zero when the constraint is not enforced and $O(1)$ when the constraint is enforced. The slack variable is typically zero when the constraint is enforced and of signficiant magnitude when not enforced. Together, they satisfy an "or" relationship.

\subsubsection{Problem 2: Objective Function}

We now state the objective function required for the second problem.

$$
f\left(\epsilon_{i}, \lambda\right)=\sum_{i=1}^{i=n} \frac{1}{\gamma_{i}}+\lambda \sum_{j=1}^{j=n-1}\left(\epsilon_{i}^{2}-n b\right)
$$

The conditions for optimal solutions to exist give

$$
\frac{\partial f}{\partial \epsilon_{i}}=-\sum_{i=1}^{i=n} \frac{1}{\gamma_{i}^{2}} \frac{\partial \gamma_{i}}{\partial \epsilon_{i}}+\lambda \sum_{i=1}^{i=n-1}\left(2 \epsilon_{i}\right)=0
$$

These equations (4-10) number $(n-1)$ altogether.

$$
\frac{\partial f}{\partial \lambda}=\sum_{i=1}^{i=n-1} \epsilon_{i}^{2}-n b=0
$$

Along with equations 4-10, we have $n$ equations altogether. Please refer to Appendix $\mathrm{D}$ for a brief review of the solution techniques used for the equations.

\subsection{Distribution of Optimal Solutions}

We shall in this section study the optimal solutions of the objective function 1 (equation 4-5).

As a preliminary investigation of the problem, we apply these optimization techniques to a system of three pendula to determine the precise structure and distribution of these optima. We first use the steepest descent method with a series of initial guesses ranging over $-.05 \leq \epsilon_{1} \leq .05$ and $-.05 \leq \epsilon_{2} \leq .05$. We focus on both objective functions. This would enable us to determine all the optima and determine the nature of the distribution of these optima in the two parameter space. The results of this study are as follows. There are six local optima shown in figure 4-1. They are 




Figure 4-1: Distribution of Local Optima for System of Three Pendula : Objective Function 1 
marked on the localization factor contour map for mode two which is the least localized mode. Interestingly, each of these optima lie roughly on the bisector between the lines of two root coalescence. In some sense these optima are situated on the points of maximum distance from the two root coalescence lines. We specified a minimum localization factor of $\gamma=2$. As we increase the minimum localization factor, we will see the optimal point move outward. The optimal solution does not necessarily lie exactly on the $\gamma=2$ curve, because there are three modes and it could be that one of the other modes is satisfying the condition for the minimum localization factor value. We next look at the distribution of optima connected with the objective function 2. These are shown in figure 4-2 superposed on the localization factor plot. These optima are those that satisfy the constraint that the mean square disorder is $5 \%$. All the optima lie on a circle however because of the constraint that the mean square disorder is $5 \%$. They also again seem to lie on the line of maximum distance from the branch point curve.

We now perform a convergence study on the steepest descent method applied to this problem. We systematically cover the entire range of parameters $-.3 \leq \epsilon_{1}, \epsilon_{2}, \leq .3$ with guesses ranging over a grid of width .05 . The convergence basin for each optima is that region of space which is demarcated by the two root coalescence lines and is shown in figure 4-3. There are six symbols on the graph representing each one of the six final solutions. Each symbol represents the final solution for an initial guess at the point in question. Those initial guesses which were positioned on or close to the two root coalescence lines resulted in final solutions which were far away from their initial guess and did not stay within the quadrant bounded by the two root coalescence lines. This was because of the local minima associated with the localization factor in those regions of the parameter space.

Three facts emerged from this study. There exist multiple optima. These optima are in quadrants of the space separated by the two root coalescence lines. The convergence basins are roughly the quadrants carved out in this space by the lines of two root coalescence. Initial guesses in the zones of large sensitivity however do not result in final solutions which are close to the initial guess. This is because the 
छ̈genvector 2



Figure 4-2: Distribution of Local Optima for System of Three Pendula : Objective Function 2 


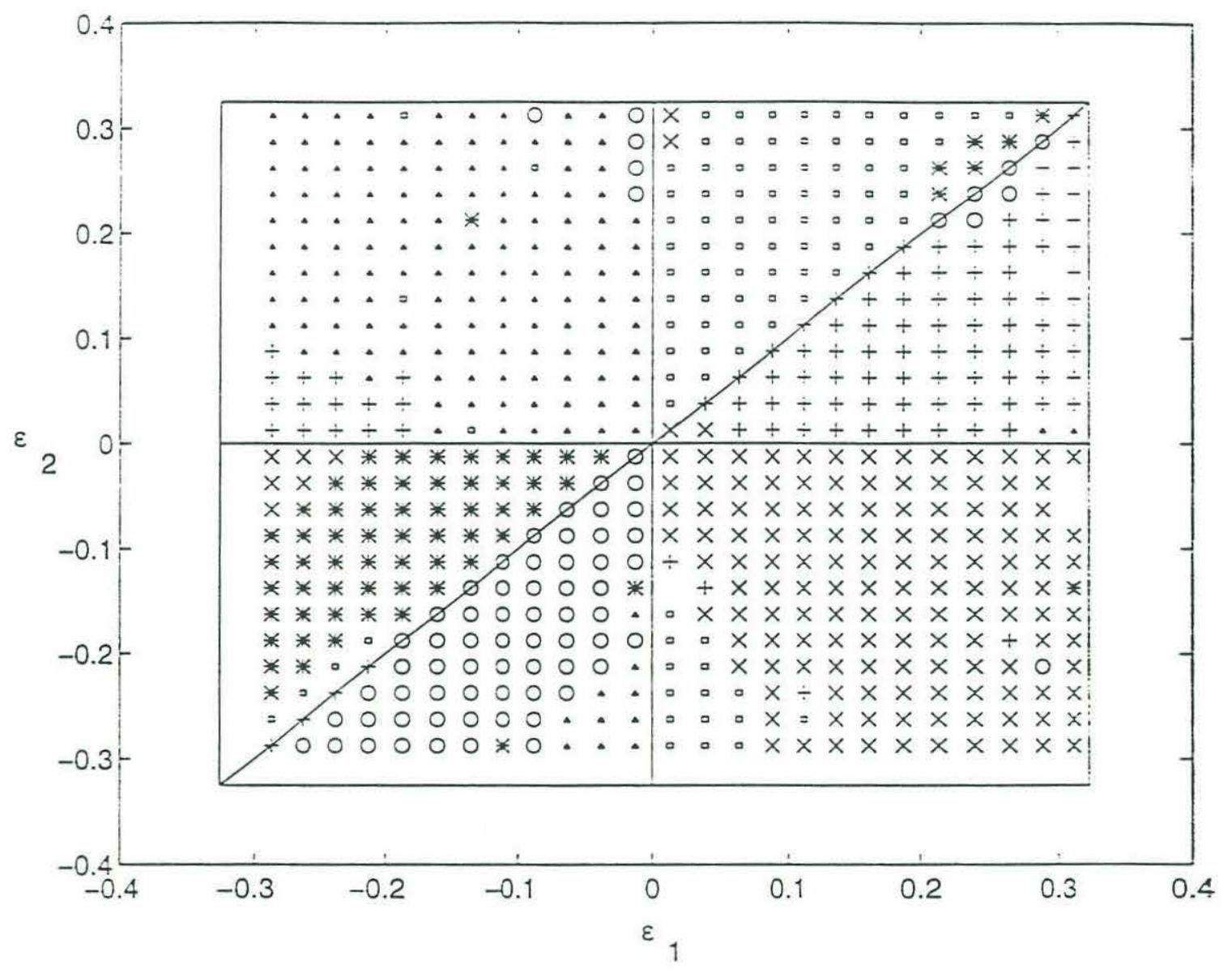

Figure 4-3: Convergence Basin for Steepest Descent . Yethod for Three Pendula 
iterated solution tends to shoot off far away from the initial guess due to the large change of gradients and the discontinuities associated with the absolute value in the localization factor definition, in this zone of the parameter space.

\subsection{Development of an Algorithm to determine the Global Minimum}

We use the knowledge of these optima gained from our preliminary analysis to develop an algorithm to determine the global minimum for objective function one.

Stage 1 : Identification of the quadrants: The $n$ dimensional space is divided into $n$ quadrants by the two root coalescence lines. The asymptotic values of these two root coalescence surfaces can be used to demarcate the parameter space into the different convergence zones. The asymptotic values that can be assumed by the disorder parameter $\epsilon_{i}$ are -1 and $\infty$. We systematically solve the two root coalescence equations for asymptotic values of the branch point surfaces. Direction cosines of these asymptotically determined points in the branch point surface are known.

Stage 2 : Initial search direction: We have already seen for the three pendulum model that the bisector to these asymptotic two root coalescence points for the least localized mode passes close to the optimal solution. Hence for the $n$ dimensional problem, the logical procedure is to first determine the least localized mode and then proceed along the bisector to the asymptotic points on the two root coalescence surface seeking to minimize the function $\left(\gamma_{\min }-b\right)^{2}$ where $b$ is the specified level of localization for the system and $\gamma_{\min }$ is the localization factor associated with the least localized mode. One issue remains. How do we identify the least localized mode in the direction of search. We do it by taking a big initial step in the direction of minimization and determining the least localized mode. In that direction of search, it would in general be correct to assume that is the least localized mode. We used

the method of golden section for the minimization procedure (See Press et al. [33]). Application of this stage would yield an estimate for the disorder which would be 
fairly close to the actual solution and lying in the convergence basin for this solution using the Steepest Descent technique.

One issue remains. The bisector to the asymptotic branch point surface should in general lead us to the correct estimate of the solution. However, in multiple dimensions, we are handicapped by the fact that while we have a multidimensional asymptotic surface in this space, we possess the directions of only isolated points on these surfaces. Evaluating bisectors of any two of these points would in general lead us to the correct solution if the points are on adjacent surfaces. If they are on the same surface or on two surfaces separated by another surface, we have a search direction which would give us an estimate of the solution which need not be meaningful. This is a shortcoming of this method. We generate a number of points which need not provide us with a correct estimate of the solution. See figure 4-4 for examples. In figure $4-4, a, b$ and c are asymptotic points on the surfaces of two root coalescence. $\mathrm{d} 1$ and $\mathrm{d} 2$ are two examples of search directions along the bisectors. $\mathrm{d} 2$ is a valid search direction while $\mathrm{d} 1$ is not valid as a search direction since it lies along a branch point surface. So any search along d1 will essentially yield meaningless results and represents wasted effort. This is an inefficiency associated with this method. A useful rule of thumb was to calculate the angle bisector between any point and $m$ of its closest neighbors. The value $m$ could be taken to be half the total number of points. Alternately, a more time consuming method would be to compute bisectors between all the points calculated. This would heighten the labor involved. We opted for the latter approach to retain accuracy.

Stage 3 : Usage of Steepest Descent Method to obtain a refined solution:

We now use estimates from the second stage as initial guesses for the Steepest Descent method. Some of these initial guesses are fairly accurate estimates of the actual solution. Some are a result of searching initially in the wrong direction but may still provide us with a correct estimate of the solution. Some of these wrong initial guesses may not converge to the correct solution. Two initial points might converge to the same solution. A lot of book-keeping effort was needed to keep track of all these possibilities. 
(b)

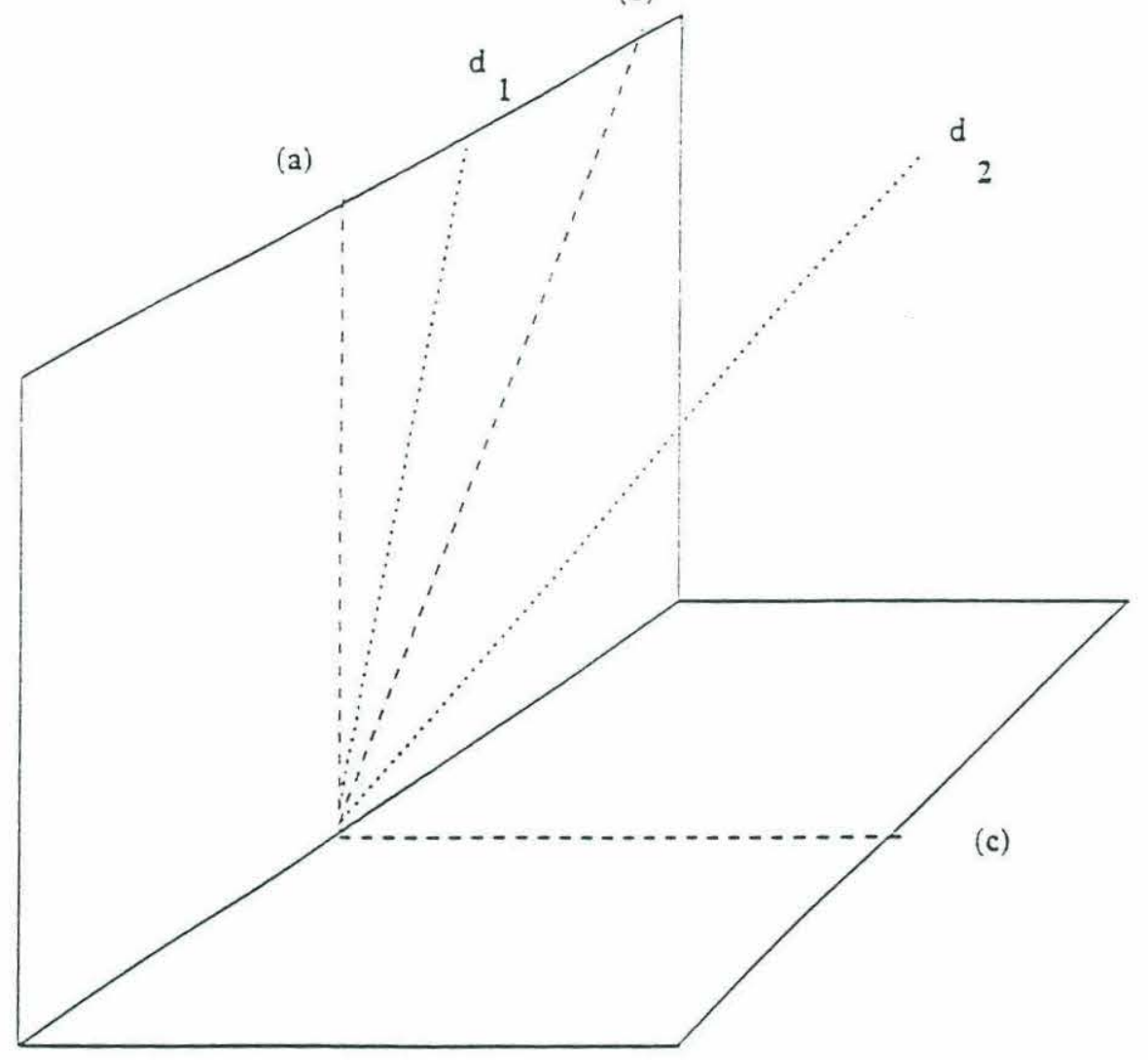

a,b,c: Asymptotic Positions on Two root coalescence surfaces.

$d_{1}, d_{2}:$ Directions between two asymptotic points on the branch point suriace

Figure 4-4: Examples of legal and illegal search directions 


\section{Distrioution of Optimal Solutions (Four Pencula)}

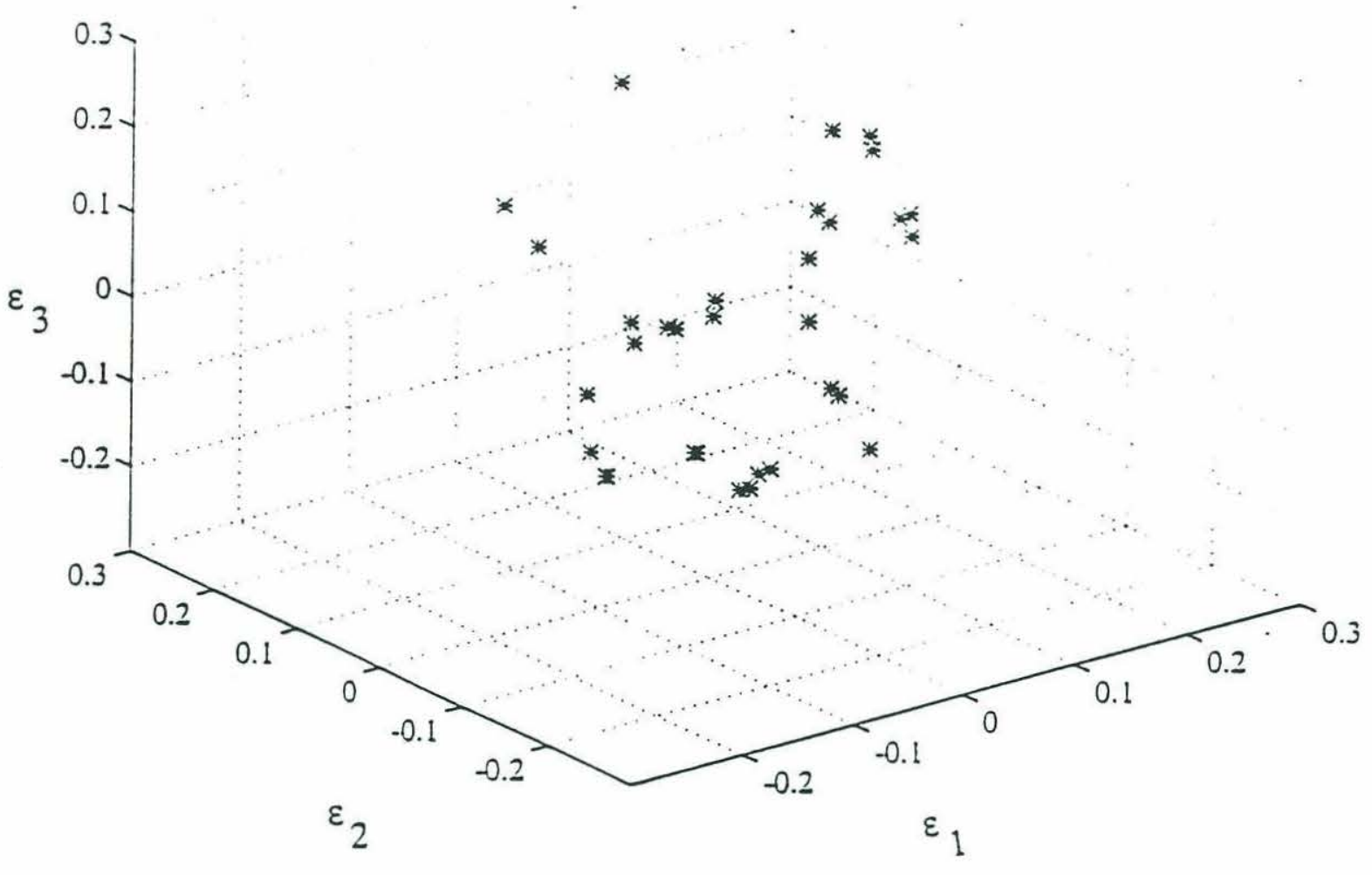

Figure 4-5: Distribution of Local Optima for System of Four Pendula 


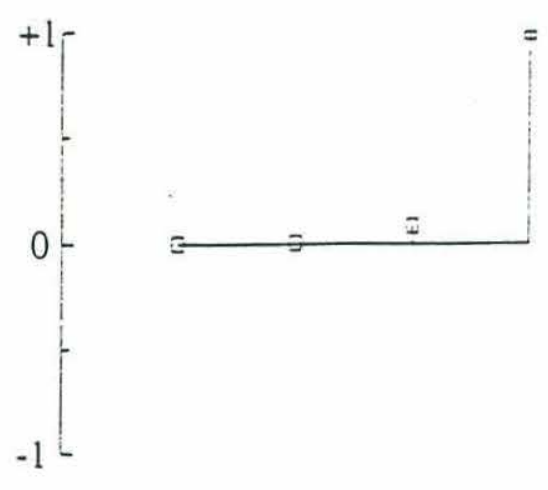

(a)



(c)

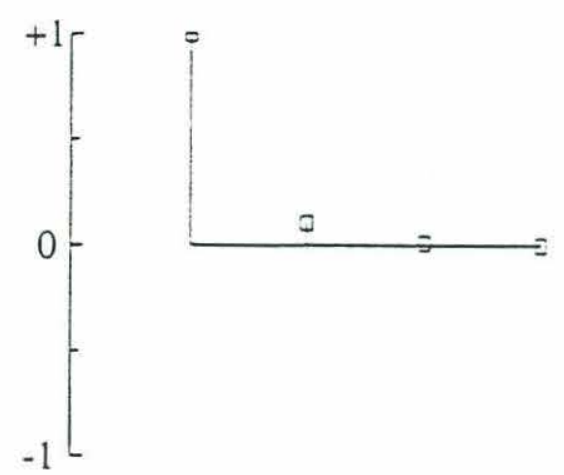

(b)

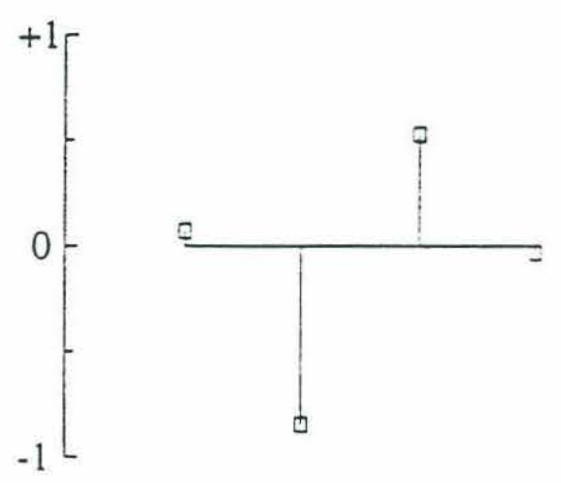

(d)

Figure 4-6: Modes at a1 for four pendula system 


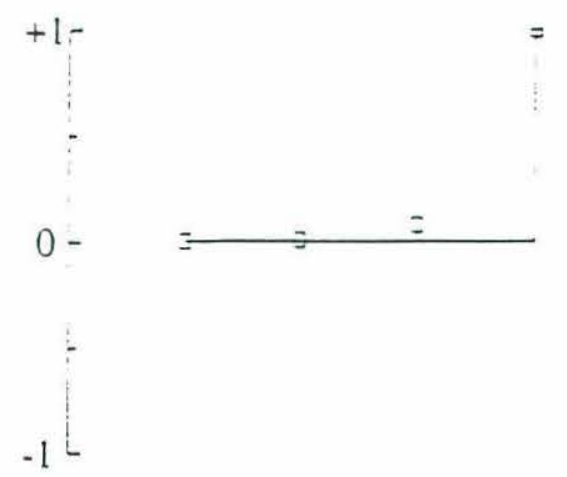

(a)

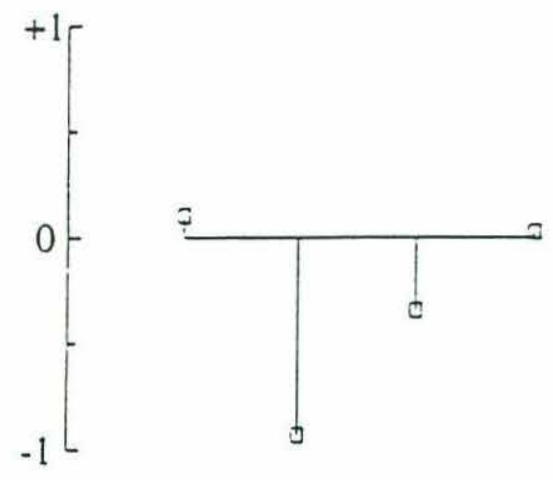

(c)

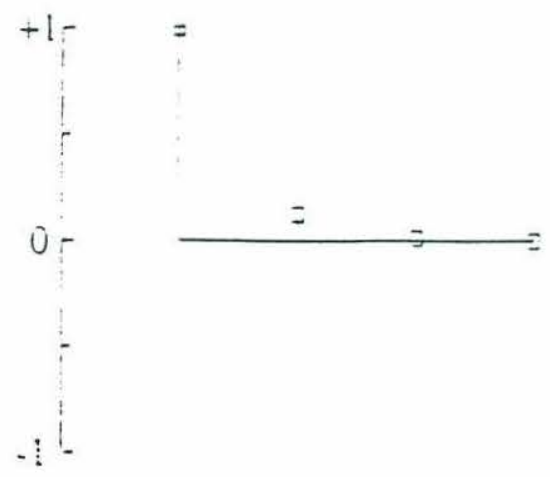

(b)

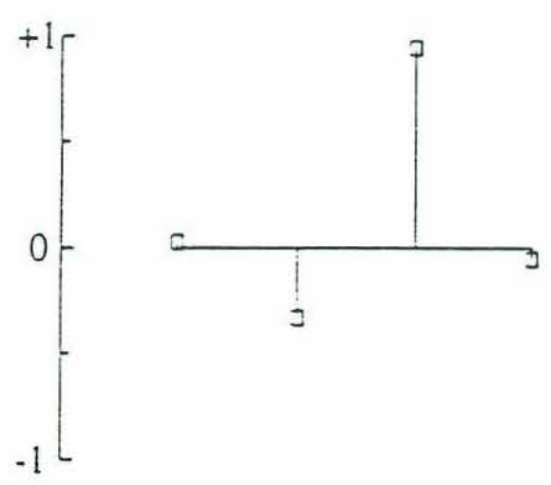

(d)

Figure 4-7: Modes at a2 for four pendula system 


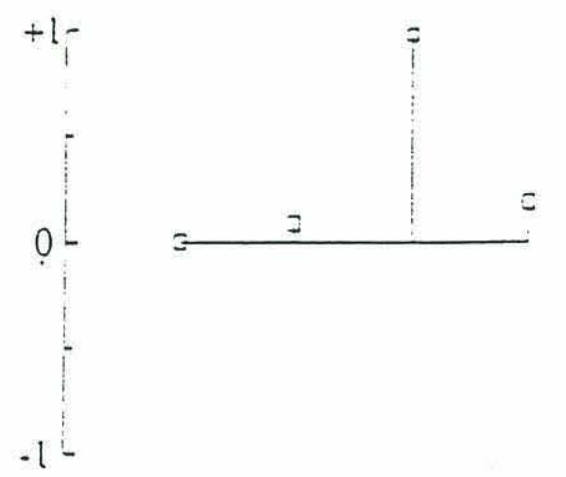

(a)



(c)

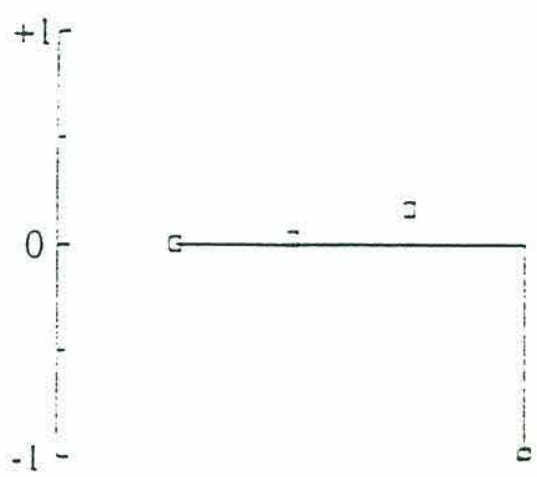

(b)

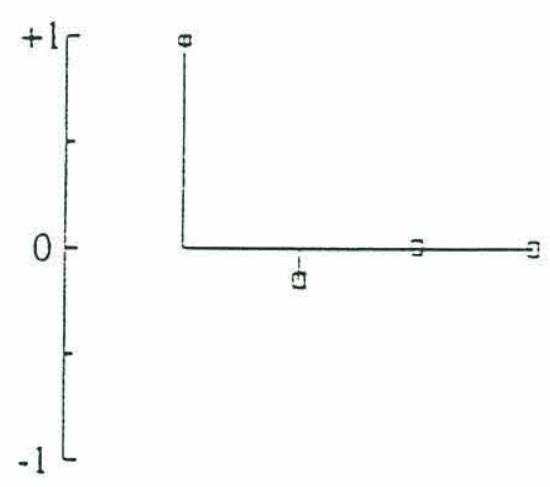

(d)

Figure 4-8: Modes at a3 for four pendula system 


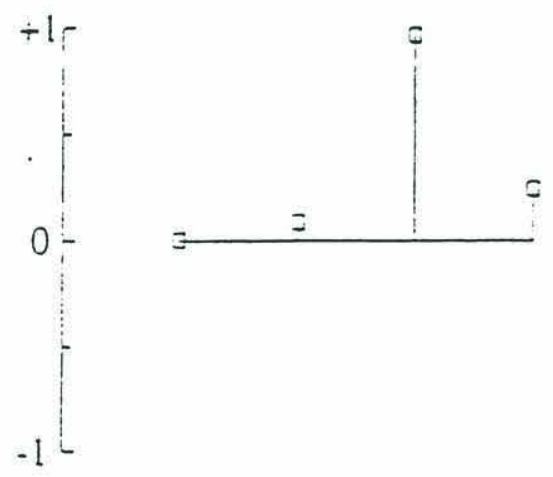

(a)

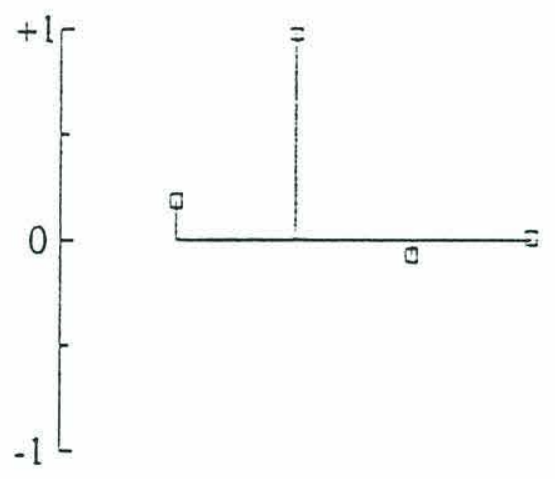

(c)



(b)

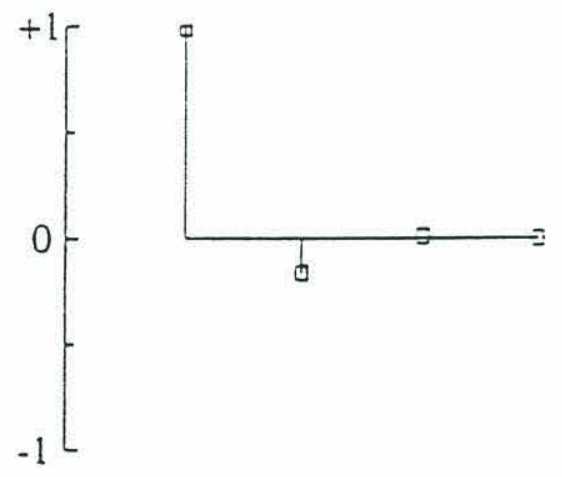

(d)

Figure 4-9: Modes at a4 for four pendula system 


\begin{tabular}{||c|c|c|c||}
\hline \hline Optimum & $\epsilon_{1}$ & $\epsilon_{2}$ & $\epsilon_{3}$ \\
\hline \hline $\mathrm{a} 1$ & -.1071 & -.1146 & -.0345 \\
$\mathrm{a} 2$ & -.1197 & -.1004 & -.0339 \\
$\mathrm{a} 3$ & .0676 & -.0563 & .1158 \\
$\mathrm{a} 4$ & .0511 & -.0687 & .1219 \\
\hline \hline
\end{tabular}

Table 4.1: Coordinates of points a1,a2,a3 and a4

We now consider a system of four pendula. We examine the spatial distribution of optima. This is shown in figure 4-5. We have tracked down all the optima using the algorithm suggested earlier. One surprising fact seen in this study is that many of the optima lie close to each other. This is surprising. We examine the mode shapes associated with some of these optimal solutions. The coordinates are shown in table 4-1. Note that (a1) and (a2) are points sharing similar coordinates but when we plot their modes in figures 4-6 and 4-7, we find that they differ in the positions of the peak of the mode. This can be explained by referring to the Chapter 2 about the mode shapes close to the surfaces of two root coalescences. We can interpret these two optima to lie on either side of the two root coalescence line. Hence they are legal separate optima. However we can have situations in figures 4-8 and 4-9 where we have optima with almost similar coordinates. These are the optima (a3) and (a4). The mode shapes in these figures are also the same. These are not separate optima and they are actually caused by round off differences in the numerical solutions.

However if our interest is the global optimum, the existence of these indistinguishable optima is not that critical. We can obtain the optima by systematically tracking down all the optima and even if there exist multiple, non-distinguishable optima corresponding to the global minimum, the error in round-off would be too small to be significant. The only issue at stake is the time taken to track down all optima. This can be very significant.

We calculated the global optimum for a system of six pendula. We uncovered 1227 


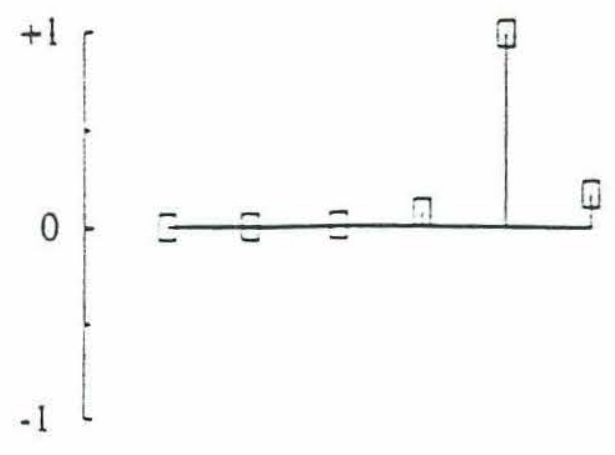

(a)

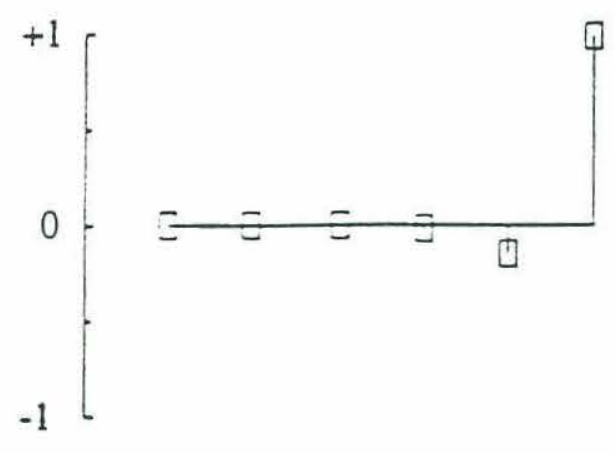

(c)

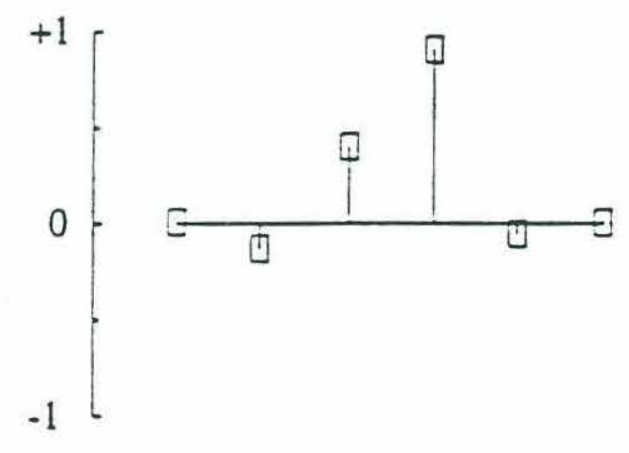

(e)



(b)



(d)

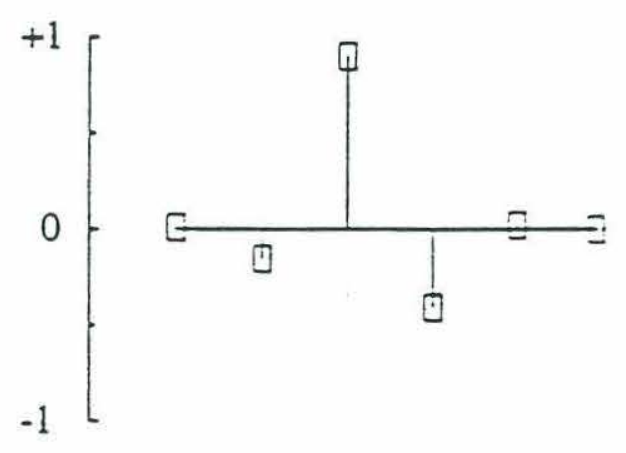

(f)

Figure 4-10: Modes at global optimum of six pendulum system, $\epsilon_{5}=.0430, \epsilon_{4}=$ $-.0486, \epsilon_{3}=-.0917 . \epsilon_{2}=-.0785, \epsilon_{1}=.0743$ 
local optima when we counted only those optima which had a distance of $1 e-06$ from each other. The modes corresponding to the global optimum are shown in figure 410. It took us 22 hours of running time on the SPARC workstation of the Design Laboratory.

\subsection{Discussion of the performance of the algo- rithm}

The algorithm for determining the global optimum is slow in terms of absolute computer time. We cannot doubt that. However it is relevant to look at the problem in a different light. The question for examining the performance of the algorithm should be posed as : How does this algorithm compare to other alternatives for obtaining the global optimum for an optimization problem?

There are very few other alternatives. We are fortunate to possess an in depth knowledge of the distribution of global optima. The only other alternative in this context is the method of Simulated Annealing and this does not promise to track down the global optimum but only promises to do so with probability one with infinite sampling of the parameter space. If we have any special knowledge of the distribution of optima, it is advisable to use that knowledge on tracking down all the optima. Computer run-times of one week are not uncommon when using the method of simulated annealing in minimization procedures for parameter estimation and this does compare favorably. However there is no doubt that this method becomes impossibly slow in estimating the global optimum for a system of say 30 pendula. This is not a shortcoming of the method as much as the fact that we have a large number of optima and these run-times are necessary to track them all down systematically.

In figure $4-11$, we provide a plot of the variation of the number of optima with the number of pendula. We provide a semi-log plot. The data for these few points appear to fall on a straight line. Thus there appears to be an exponential increase in the number of optima with the number of pendula. Now, we should note that this 
Variation of No. of Optima with No. of Pendula

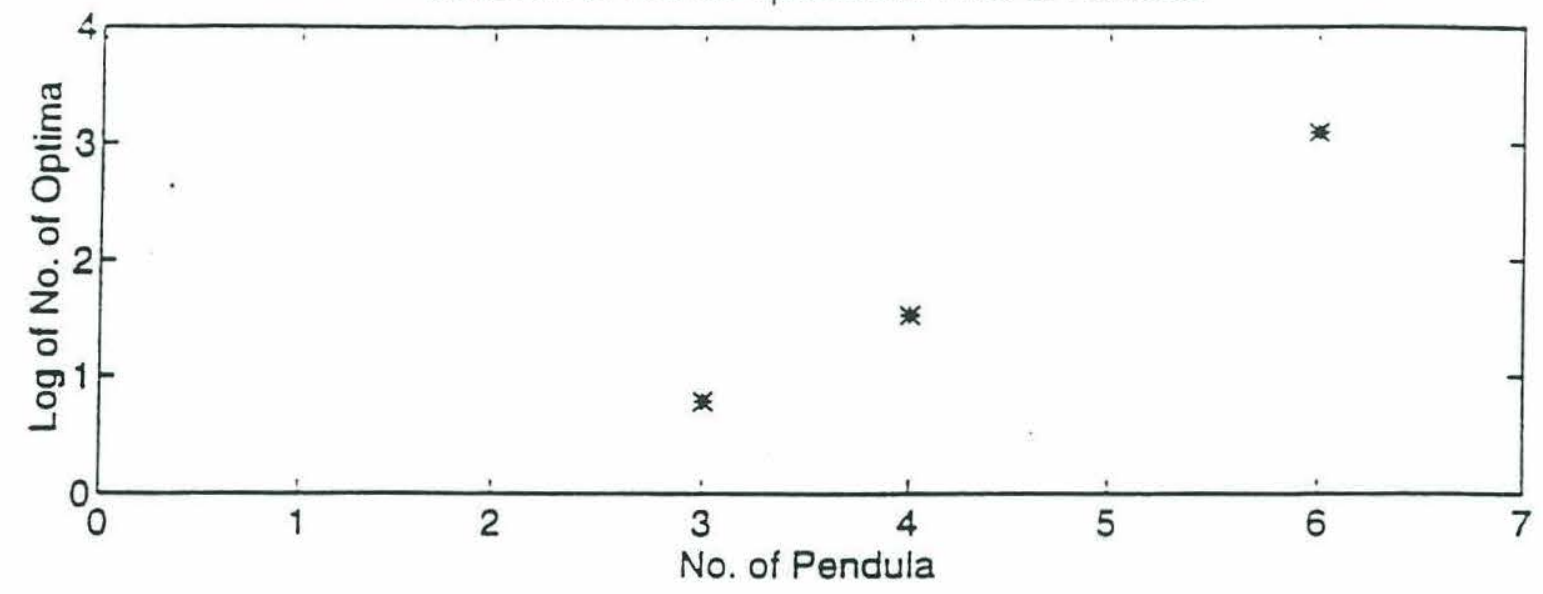

Figure 4-11: Plot of Variation of Number of Optima with Number of Pendula 
is only a trend and it would be reasonable but not absolutely correct to assume that this trend would continue for very large problems. Thus, if we denote the number of pendula by $N p$ and the number of optima by $N o p$.

$$
N o p=e^{4.1258+1.7702(N p-3)}
$$

An exponential growth of the number of optima implies that the reason for the computational effort increasing so rapidly with the number of pendula might be due to the number of optima rather than the time taken to uncover each optimum.

\subsubsection{Operation Count}

We provide an estimate of the operation count for large $N$ for confirming our suspicions of the growth rate in computational effort being due to the number of optima rather than the optimization algorithm itself. We will make a preliminary estimate assuming out initial search direction algorithm operates with $100 \%$ efficiency i.e. the initial direction results in a guess close to an optimum and the final solution is never duplicated during the entire process.

- Number of pendula $=N p$

- Number of disorder units $n=N p-1$.

Note the following operation counts :

- Operations for determination of modes of a size $N * N$ matrix: $O(3-5 N)$ using Q-Z algorithm.

- Operations for LU back substitution for size $N * N$ matrix : $O(N)$.

- Operations for LU decomposition for size $N * N$ matrix : $O\left(N^{2}\right)$.

We now use this method to determine the operation count for the various algorithms. Approximations for large $N p$ are introduced everywhere. The following operation counts are made per optimum solution The operation count for the initial search follows : 
- Average Number of Iterations $\simeq 50(\mathrm{~Np}-1)$

- Number of function evaluation per iteration $\simeq 3$

Total average number of function evaluations $=150(N p-1)$. Operations per function evaluation $=5 N p+(2 N p+1) N p \simeq N p^{2}$. The second term is the effort for actual estimation of $N p$ localization factors for each mode. Total number of operations $\simeq$ $150 N p^{3}$. The Steepest Descent has the following significant statistics :

- Maximum iterations $=100 \mathrm{~Np}$

All the following calculations are for one iteration. On average, we can expect $10 N p$ iterations. Operations in determining the Jacobian : $2\left((3 N p-1)^{2}+2 N p\right) \simeq N p^{2}$. The first $N p^{2}$ denotes effort in calculating the localization factor and the other terms like $N p$ denote the effort in other multiplications and divisions. The factor of two exists because the Jacobian is calculated by finite difference. Operations in LU decomposition: $(3 N p-1)^{2} \simeq N p^{2}$. Operations in back substitution: $3 N p-1 \simeq N p$. Operations in linesearch per iterations: $N p(3 N p-1)^{2} \simeq N p^{3}$. Summing and multiplying by the number of iterations, we get total operation count to leading order $\simeq N p^{4}$.

There are Nop optima. Hence we get the total effort to be $e^{4.1258+1.7702(N p-3)} N p^{4} \simeq$ $e^{4.1258+1.7702(N p-1)}$. Thus the exponential term dominates the effort for computation. Any decrease in the number of optima sampled would make a significant reduction in compute time.

We should now make a more complete analysis noting that we have not included the computational effort to calculate the initial search directions in this analysis. We note that we solve the two root coalescence equations for the $N p$ pendula problem to determine the search directions.

We will first determine the operation count for determining the two root coalescence asymptotes. There are two asymptotic values $\epsilon_{i}=\infty,-1$. Thus we have a total of $N$ tot $=2^{N p-2}(N p-1) \simeq e^{1.3863 N p}$ asymptotic values. Note $N$ tot is an exponential function of the number of pendula. The effort for determining the solution can be 
shown to grow as $N p^{2}$. We calculate the bisectors to $N$ tot $(N t o t-1)$ combinations to get the same number of search directions. We thus get a total of

$$
\simeq N \text { tot }+N \text { tot }(N t o t-1) 150 N p^{3} \simeq e^{1.3863 N p} N p^{3} \simeq e^{1.3863 N p}
$$

to leading order which is also exponential. We should now note that a big fraction of these search directions results in meaningless results.

So this analysis spells problems in the sense that the computational time grows exponentially with the number of pendula. However the next question is can we perhaps by sampling fewer of the optima still obtain a good estimate of the global optimum ? Obviously since there is an exponential growth in computational effort, if we examine a sufficiently large system(like say eighteen to twenty pendula), we will find it impossible to track down all the optima and then estimate the global optimum. But this reduced sampling would still make the method viable for small systems of two to ten pendula. The effect of any such analysis would be two-fold. Firstly, we would only have to sample a fraction of the the points for estimating the global optimum. Also, we would only be required to estimate a fraction of the asymptotic directions which are used to determine the initial guesses for the Gauss-Newton search.

\subsection{Statistical Analysis of the Distribution of Op- tima}

The first step in the analysis is to determine whether there is a sufficient spread in the optima to warrant determining all the optima and thence the global optima. Hence, it is instructive to examine the distribution of optimal solutions. In figure 4-12, we provide a histogram plot of the distribution of the Root Mean Square (RMS) disorders of the optimal solutions for six pendula. Note the following :

- The distribution is one-tailed.(If there is a minimum, the distribution has to be one tailed). 


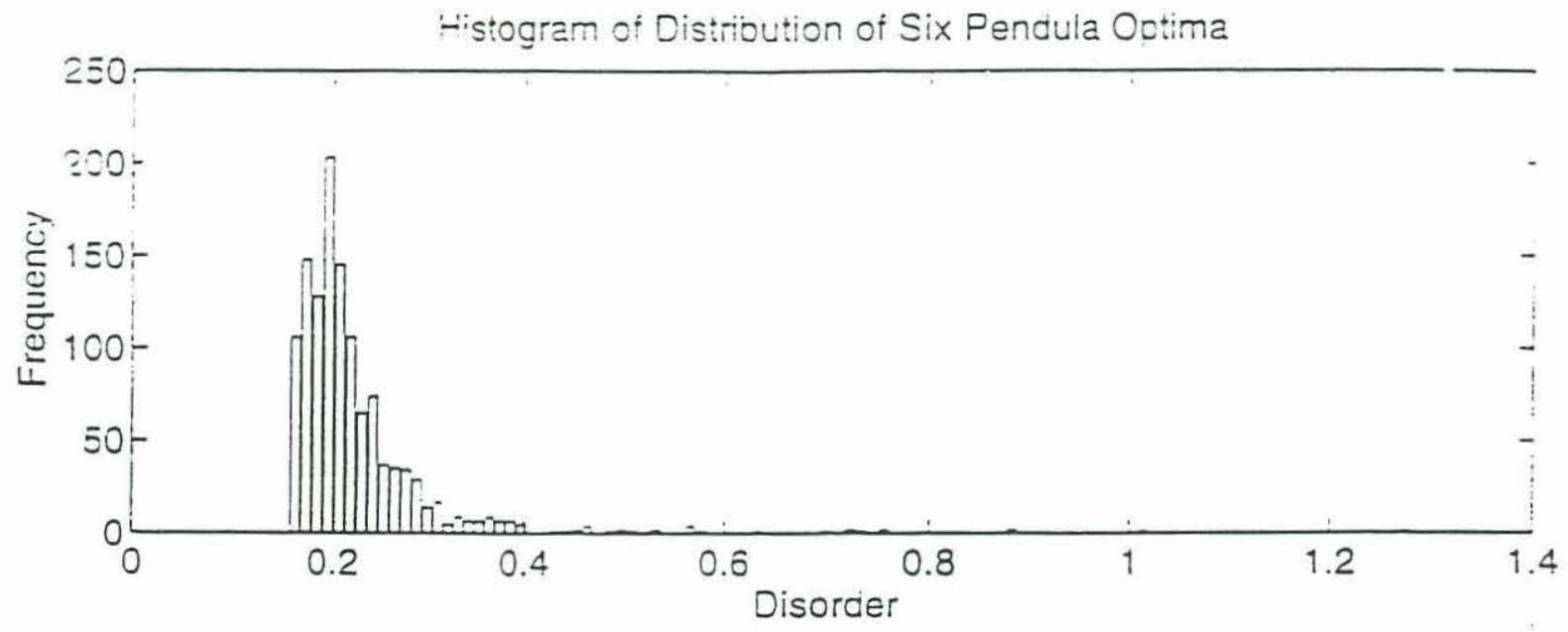

Figure 4-12: RMS disorder distribution for local optima for six pendula system 
- The distribution is skewed and has a long tail.

For the sake of completeness, there is no doubt that we need to compute the global minimum. Is there a sufficiently large difference between the global optima which have the greatest mean square disorder(GMSD) and least mean square disorder(LMSD) to warrant the extra effort to calculate the optimum? We plot histograms of the distributions of mean square disorders which we obtained for systems of three, and four pendula in figures 4-13 and 4-14 also. In figure 4-12, we see clearly that there is a ratio of about five between the GMSD and LMSD. But there is a band close to the LMSD where most of the optima are located. In figure 4-13, we see that the band is still there close to the LMSD but the ratio between the GMSD and LMSD is about two. In figure $4-14$, we see that the band is broad and is again located close to the LMSD but the ratio between the GMSD and LMSD is about two. So while the outliers in this distribution are well separated, the vast majority of the optima are closely spaced in a band close to the LMSD. If we ensure that any optimum we select falls in this band, we will obtain a very good selection of the optimal solution. So if the aim is to gain a good estimate of the global optimum rather than the exact global optimum, a good estimate would be obtained by sampling a few of these local optima and taking the least of that selection. We would then be no longer in the region of the long tail. The question to be answered is how many optima do we need to sample to ensure our estimate of the minimum falls in the band where most of these optima lie?

This falls in the domain of order statistics. Let us consider the distribution of the RMS disorder $D$. Let it possess a probability density function $f(D)$. The cumulative density of the RMS disorder of these optimal solutions is $F(D)$. Let us now attempt to determine the CDF $G_{m}\left(d_{m}\right)$ and PDF $g_{m}\left(d_{m}\right)$ of the random variable denoted by $d_{m}=\min \left(D_{i}\right)$ where $1 \leq i \leq m$. This random variable represents the global minimum of a sample of size $m$.

We follow the development in Drake [13] and Lass and Gottlieb [21] where the $\mathrm{CDF}$ of the minimum of a sample of size $m$ is given by 


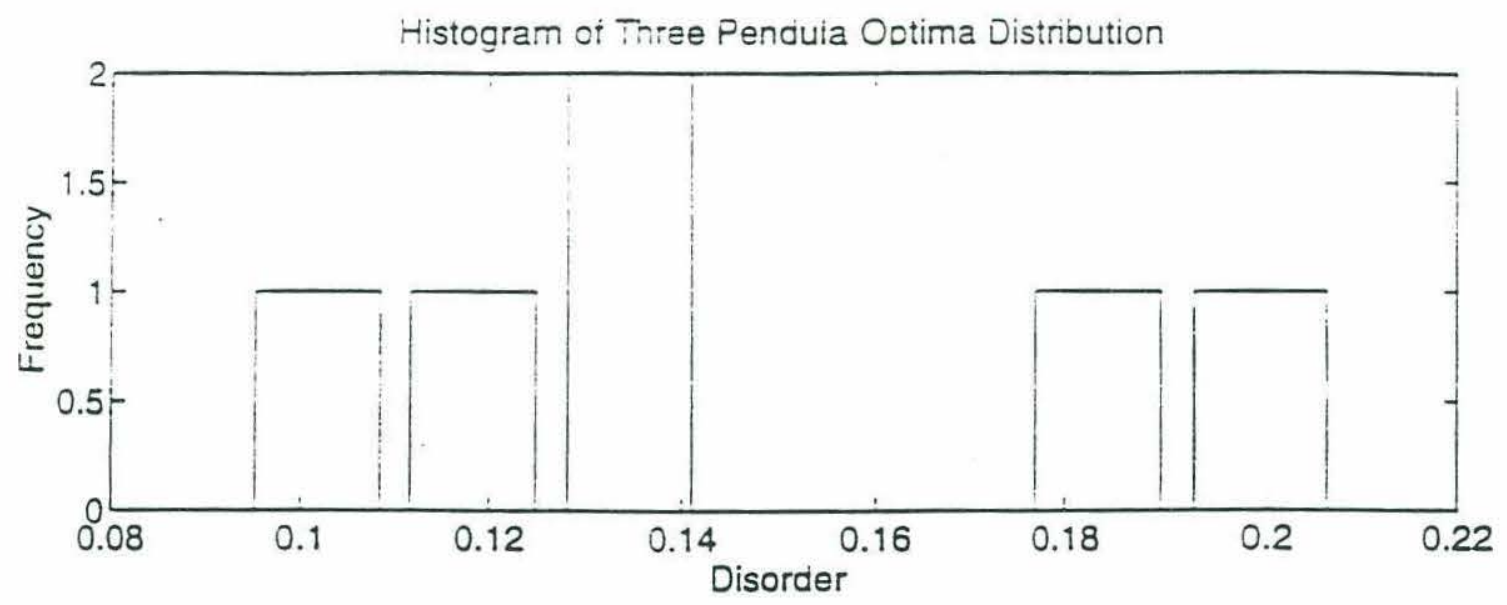

Figure 4-13: R.MS disorder distribution for local optima for three pendula system 


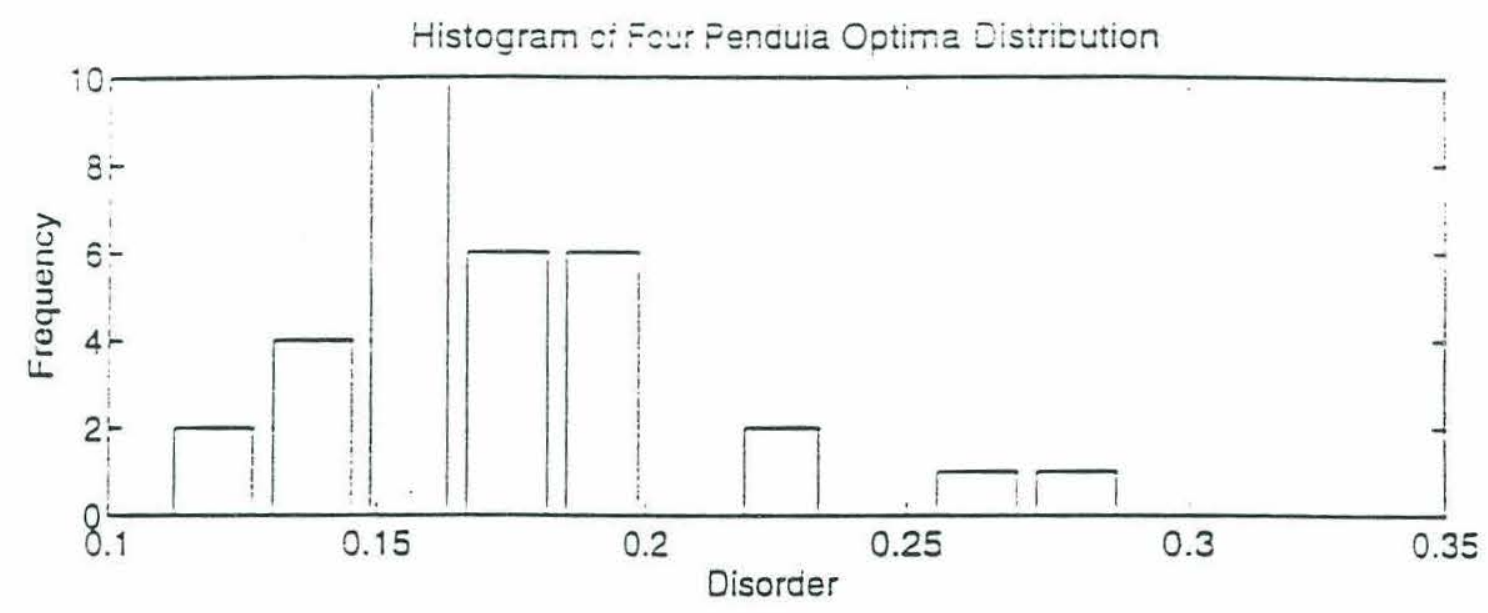

Figure 4-14: RMS disorder distribution for local optima for four pendula system 


$$
G_{m}\left(d_{m}\right)=1-\left(1-F\left(d_{m}\right)\right)^{m}
$$

and the PDF is given by

$$
g_{m}\left(d_{m}\right)=m\left(1-F\left(d_{m}\right)\right)^{m-1} f\left(d_{m}\right)
$$

Our illustrative example is the distribution of optima for the six pendula system. We fit a Weibull distribution to the data. The choice of this is based on two reasons. The first reason is that the Weibull distribution has historically been used on data for weakest link in a chain type problems where the chain with the minimum strength has to be identified. This is very parallel to this problem in that the statistic of interest is the minimum value of the parameter. The second reason is that the data is one tailed with a well defined cut-off and has a skew to the left. This can be handled by the Weibull distribution. We should note that there are other distributions which can capture the features of the data. The general results viz. that the minimum of a sample of a few of these optima provides a good estimate of the minimum, is however independent of the fitted distribution.

\subsubsection{The Weibull Distribution}

The three parameter distribution is defined by the parameters $\mu, \sigma$ and $\lambda$. If $D$ be the random variable which represents the RMS disorder of any optimal point, we define $x=D-\mu$. The CDF is defined by

$$
F_{W}(D, \mu, \sigma, \lambda)=1-\exp \left(-\left(\frac{(D-\mu)}{\sigma}\right)^{\lambda}\right)
$$

where $\mu \leq \sigma,-\infty \leq \mu \leq \infty .0 \leq \sigma, \lambda$.

We determine if the data is indeed Weibull distributed by plotting the data on a double log paper. In figure $4-15$, we provide plots of the data without the cutoff $\mu$ being incorporated into the model. Clearly, the data is skewed. This is a test to determine if the data has a Weibull distribution with two parameters. If the data 


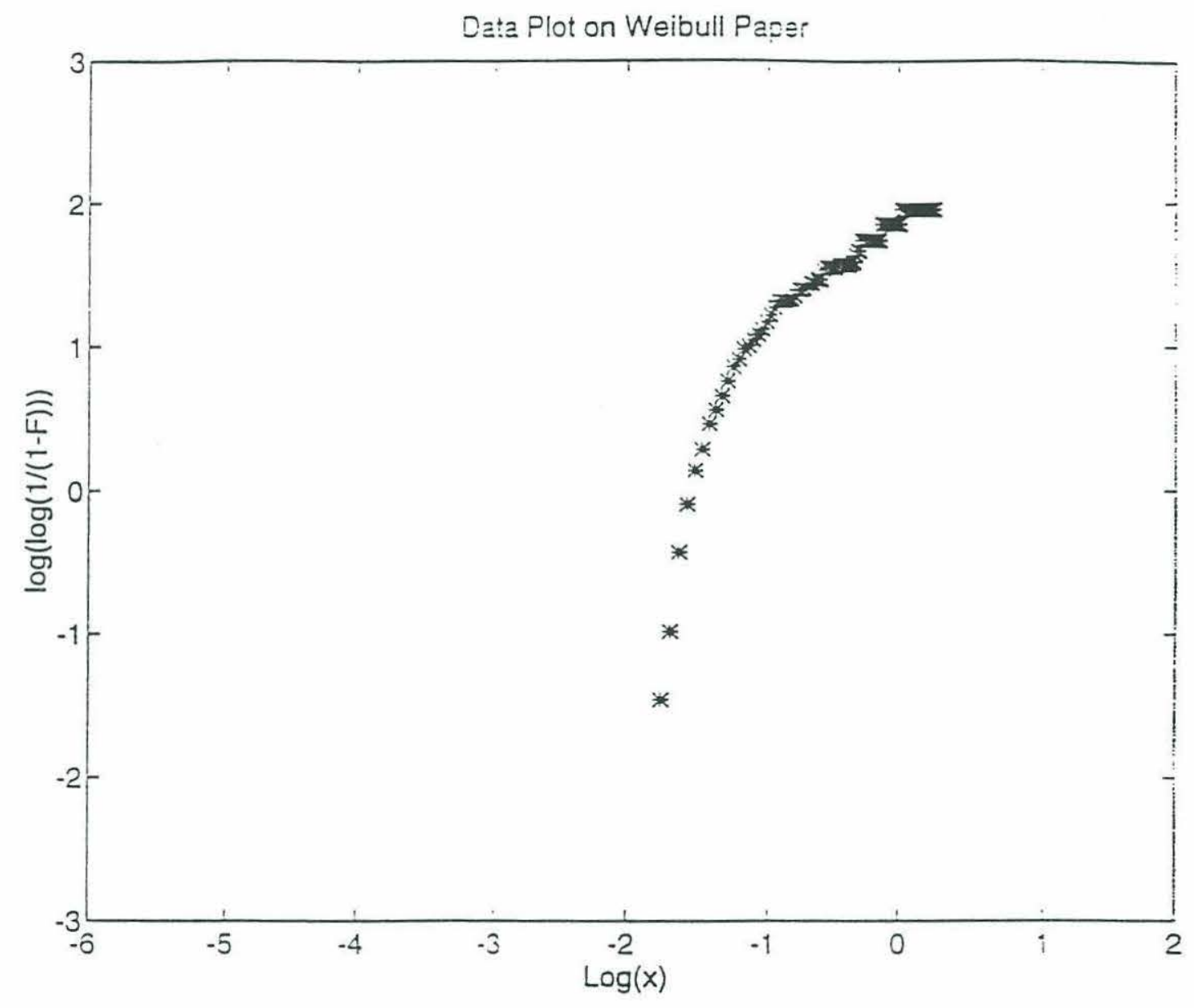

Figure $\div 15:$ RMS data plot on Weibull graph without cutoff 


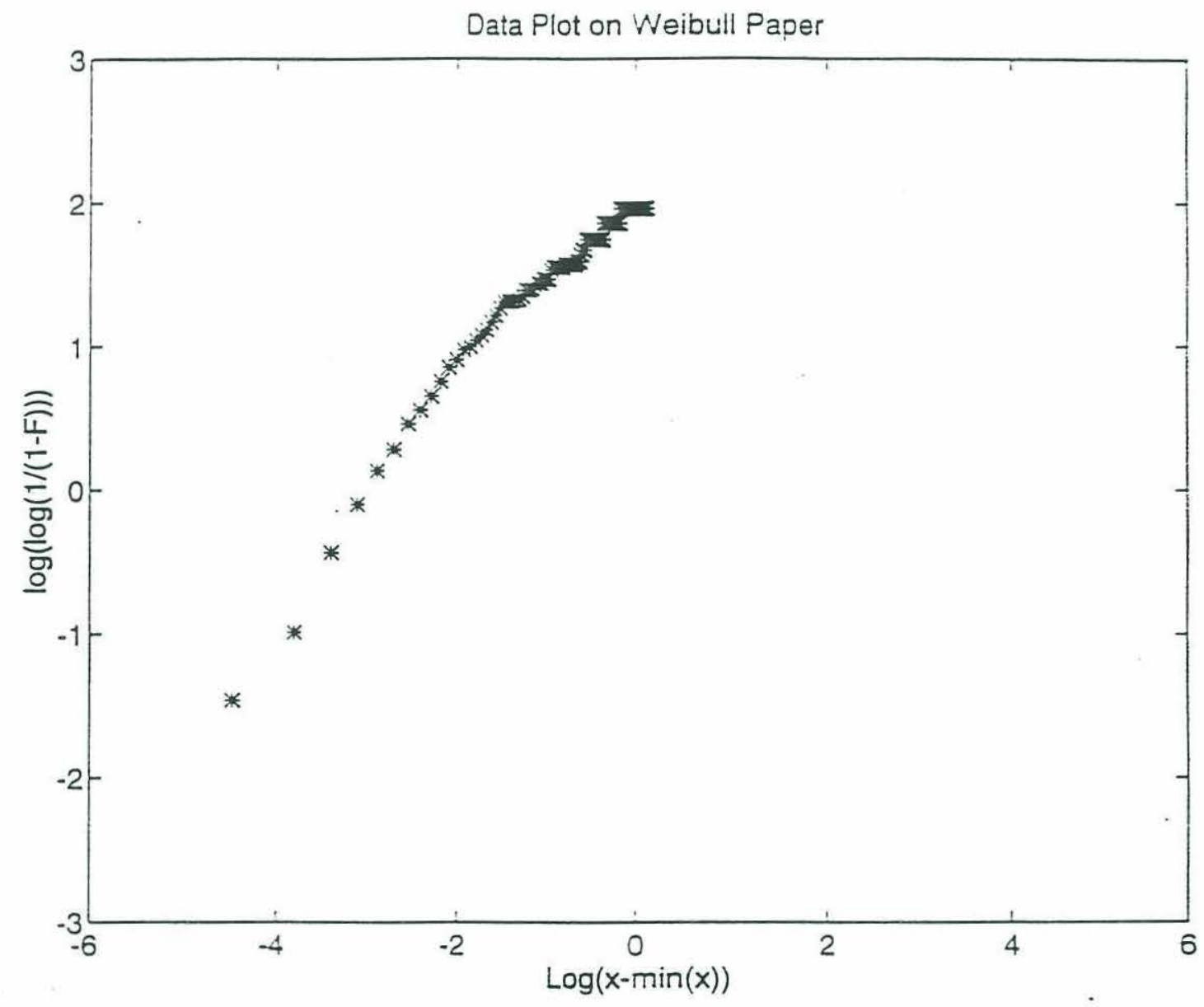

Figure 4-16: RMS data plot on Weibull graph with cutoff 
were distributed as a Weibull distribution of two parameters, we would have the data distributed as a straight line. In figure 4-16, we provide a plot with the cut-off $\mu$. The cutoff $\mu=\min (D)$. The data are scattered along a straight line without a skew. This is a graphical test to determine whether we use a three parameter Weibull distribution. Since the data is scattered roughly in a straight line, we can conlude that it is correct to use a three parameter Weibull distribution The y-intercept of the plot is $-\lambda \log (\sigma)$ and the $\mathrm{x}$ intercept of the plot is $\sigma$. However the graphical procedure is only used for a check and for actual estimation of the parameters we will use the maximum likelihood equations from Bury [8]. These equations are as follow :

$$
\begin{gathered}
\frac{n}{\lambda}-n \log (\sigma)+\sum_{j=1}^{j=n} \log \left(D_{j}\right)-\sum_{j=1}^{j=n} \frac{D_{j}^{\lambda}}{\sigma^{\lambda}} \log \left(\frac{D_{j}}{\sigma}\right)=0 \\
\left(\sum_{j=1}^{j=n} D_{i}^{\lambda} \log \left(D_{j}\right)\right)\left(\sum_{j=1}^{j=n} D_{j}^{\lambda}\right)^{-1}-\frac{1}{\lambda}=\frac{\sum_{j=1}^{j=n} \log \left(D_{j}\right)}{n} \\
\sigma=\left(\frac{\sum_{j=1}^{j=n}\left(D_{j}-\mu\right)^{\lambda}}{n}\right)^{\frac{1}{n}}
\end{gathered}
$$

They can be easily solved numerically using some iterative method to obtain the solution. A plot of the Weibull Distribution is shown in figure 4-17. It recaptures the long tail and skewed nature of the original data. We now return to figures 4-13 and 4-14. In both cases we see the same broad features of a skewed peak and a long tail. This is a check. We wish to establish beyond any doubt that the results of the statistical analysis are valid for different numbers of pendula by checking if a trend exists in smaller systems of pendula for the distribution of optima to remain similar regardless of the number of pendula. We will assume that the distribution obtained for six pendula is roughly what will be seen for larger systems of pendula.

We now determine the $90 \%$ confidence interval for the minimum of samples of size $m$ as $m$ increases in size. This analysis has however made some rather strong assumptions about the optimal solutions. We assumed that the RMS disorders of the optimal solutions are random variables which are independent of each other. In other words, selecting say the first three optima did not affect the distributions of 
Fitted Weibull Distribution

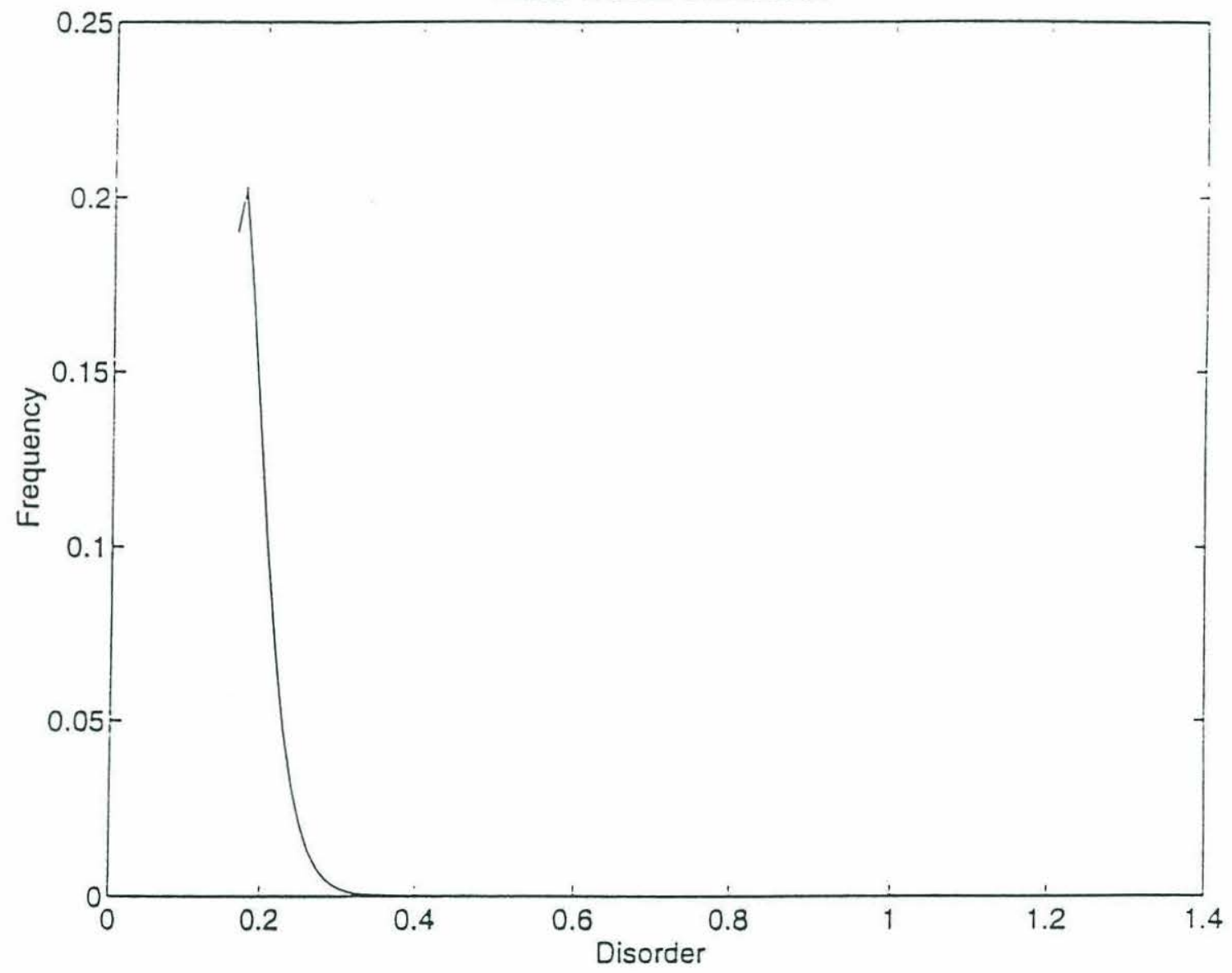

Figure 4-17: Fitted Weibull Distribution 
the other optima. This need not be correct, especially for small sample sizes but it is a reasonable assumption. In figure $4-18$, we provide plots of the $90 \%$ confidence intervals for the minimum of a sample of size $n$ as a function of the size of the sample. The $90 \%$ confidence interval is

$$
\left(d_{n}\right)_{.9}=\mu+\sigma\left(\frac{-\log (.1)}{n}\right)^{\frac{1}{\lambda}}
$$

We see that sampling as few as $10 \%$ of the optima, provides us with a good estimate of the global optimum (within 2\%). This on first sight appears contrary to our intuitive expectations viz. that in the histogram shown in figure 4-12 we needed to count at least 100 samples to ensure we were out of the tail of the distribution, and perhaps 800 before we were truly close to the global optimum. However we should note that this is not true because we are making the assumption that a sample of size much smaller than the population would essentially reflect the distribution of the whole population. In other words, the chances are that we will not sample the optima in the order where we first cover all the optima in the tail and work our way to the global optimum. The question then is whether the numerical algorithm we are using also tracks down the optima in accordance with this assumption. We answer this by actually sampling the optima in the order in which the algorithm uncovered them and plotting the minimum of the sample as a function of sample size. This is superposed in figure 4-18 on the predicted confidence intervals calculated from the distribution. The actual data shows good agreement with the predictions from the distribution. Obviously, this is only one combination of the data. Our analysis indicates that $90 \%$ of the combinations of data would have global optima of samples of various sizes lying within the indicated curve. We have taken some pains to explain this concept because in our mind, this is an area which readily lends itself to misinterpretation of results.

We note that when we examine a different system of pendula than $n=6$, the parameters $\mu, \sigma$ and $\lambda$ will change. The estimate requiring $10 \%$ of the optima to be sampled to obtain an estimate within $2 \%$ of the global optimum would continue to remain true if $\sigma$ and $\lambda$ do not vary too much. $\lambda$ is a parameter which determines the 


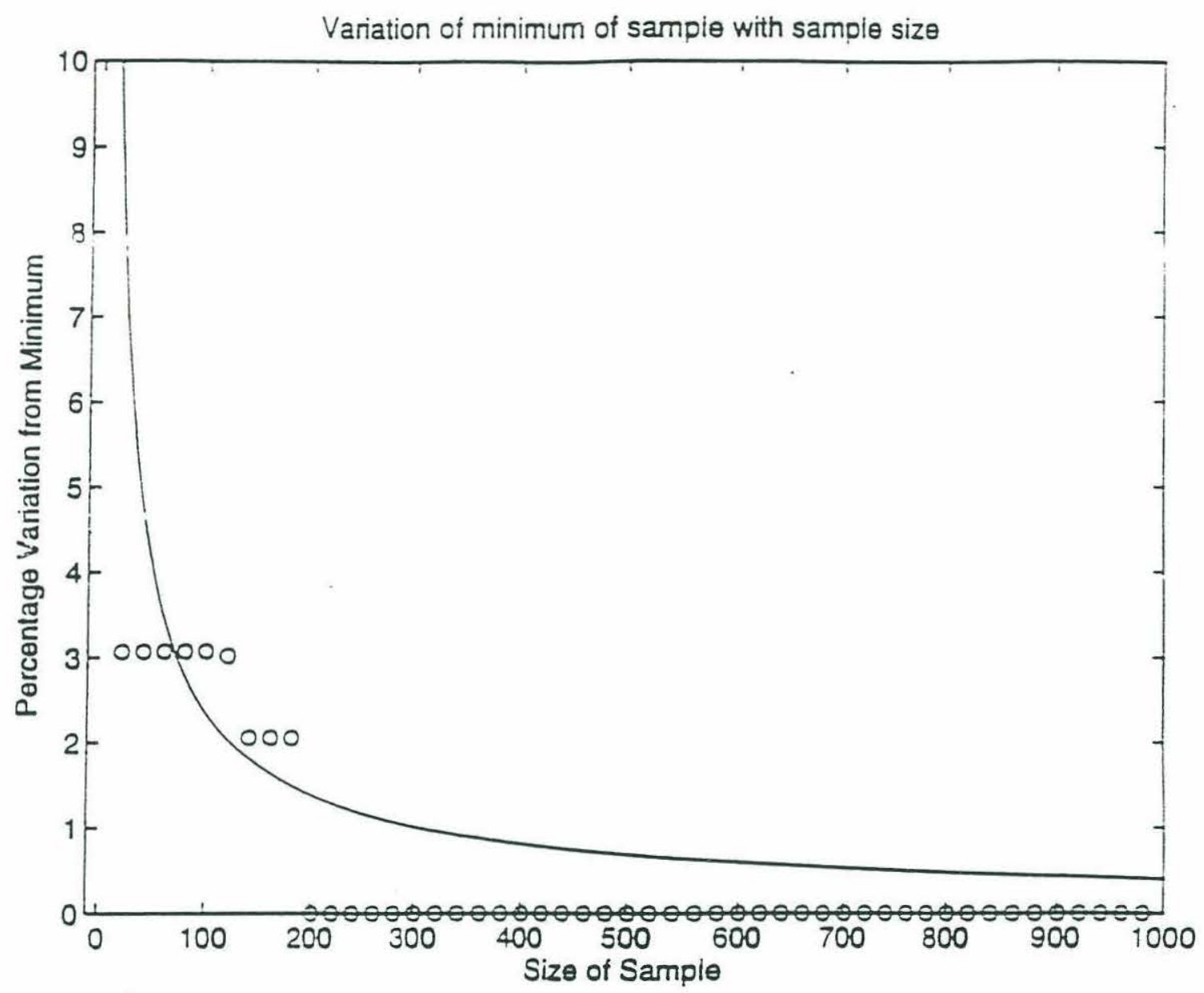

Figure 4-18: Minimum of sample(expressed as a percentage of global minimum) vs. sample size.-: Confidence Bound from Weibull Distribution. ॰: Actual Minimum. 
extent of skew and $\sigma$ is a parameter which determines the spread of the distribution. As we have seen, over the histograms associated with different numbers of pendula, these characteristics remain reasonably constant over different numbers of pendula. Hence it is reasonable that this result would hold true for different numbers of pendula too.

\subsection{Optimal solutions for larger systems of pen- dula}

We provide optimal solutions for the system of ten coupled pendula for objective functions 1 and 2 (equations 4-5 and 4-9). We note that we have only found a local optimum for the system of ten pendula. The modes are plotted in figures 4-19 and 4-20. Clearly, the modes associated with the objective function two, appear to have the same level of localization as those associated with the objective function one even though at first glance, the RMS disorder appears to be far larger for the first objective function. This is because we have not found the global optimum. However, the fact that two of the oscillators only have significant amplitude indicates that we are close to a two root coalescence surface in the 10 dimensional space.

Note that we have not even tried to locate the global optimum because of the large requirements of computational time. Hence we have restricted ourselves to merely finding local optima which do satisfy the equations for optimality.

In theory, we can apply this method to very large systems of coupled pendula. However, we found that the method of steepest descent converged to optimal solutions only for $n \leq 15$. For larger systems of coupled pendula. we actually found the method did not converge. This was initially mystifying. But we were successful in finding out the reason for the non-convergence of the method. In figure 4-21, we plot the localization factor as a function of disorder in the left-most pendulum for a three pendula system and thirty pendula system and in figure 4-22 we show the logarithm

of the mode shape associated with a localized mode of a thirty pendula system. The 


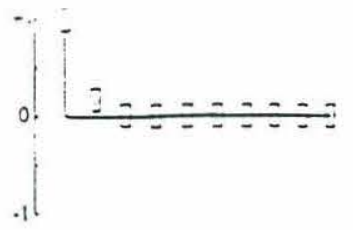

(a)

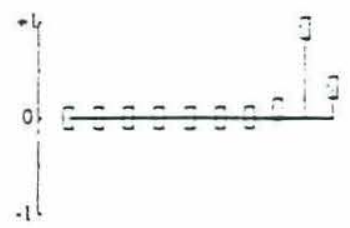

(c)

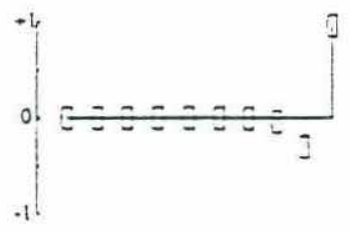

(e)

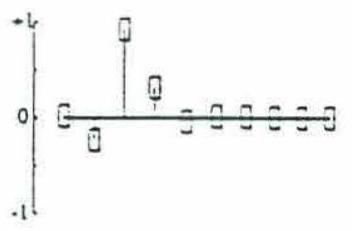

(z)



(i)

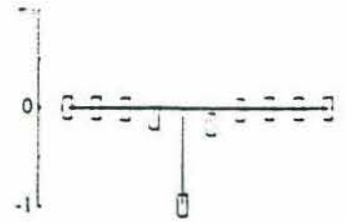

(b)

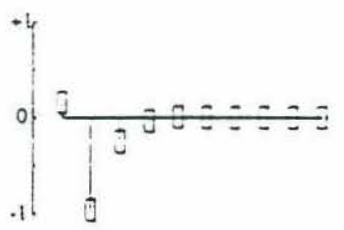

(d)

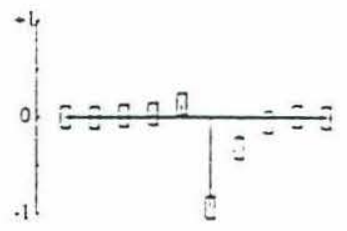

(f)

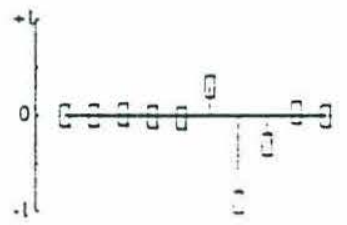

(h)



(j)

Figure 1-19: Modes of ten pendulum for objective function 1: $\epsilon_{9}=.201, \epsilon_{8}=$ $.1029, \epsilon_{7}=.0434, \epsilon_{6}=.1599, \epsilon_{5}=.0211, \epsilon_{4}=.1992, \epsilon_{3}=.1992, \epsilon_{2}=.0797, \epsilon_{:}=.2299$. RMS disorder $=.1436$ 


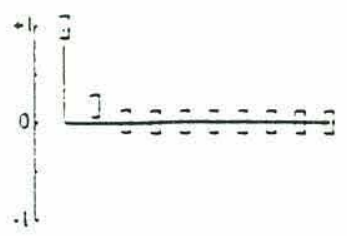

(2)

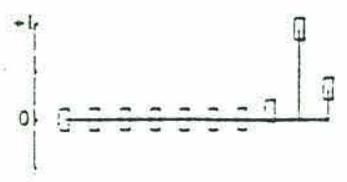

.16

$\therefore:$

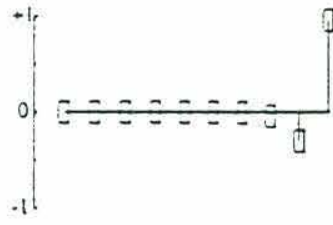

(e)

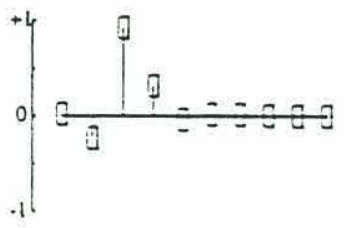

(3)

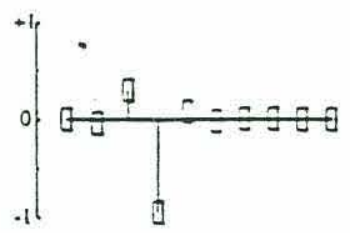

(1)



(b)

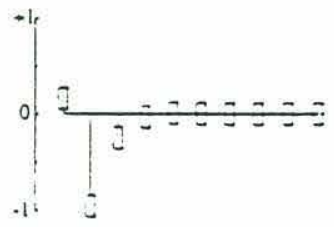

(d)

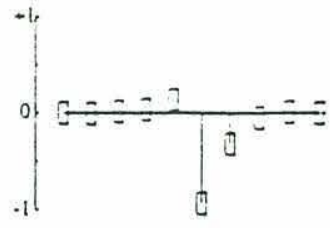

(n)

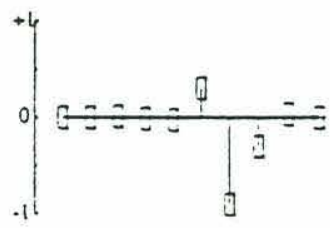

(h)

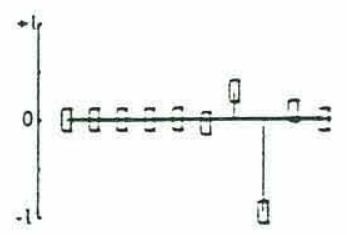

(j)

Figure 4-20: Modes of ten pendulum system for ten pendula for objective function $2: \epsilon_{9}=.0613, \epsilon_{8}=.0101, \epsilon_{7}=-.0296, \epsilon_{6}=-.0554, \epsilon_{5}=.0519, \epsilon_{4}=-.0149, \epsilon_{3}=$ $-.0431, \epsilon 2=-.0698, \epsilon_{1}=.0375$, R.IS disorder $=.05$ 

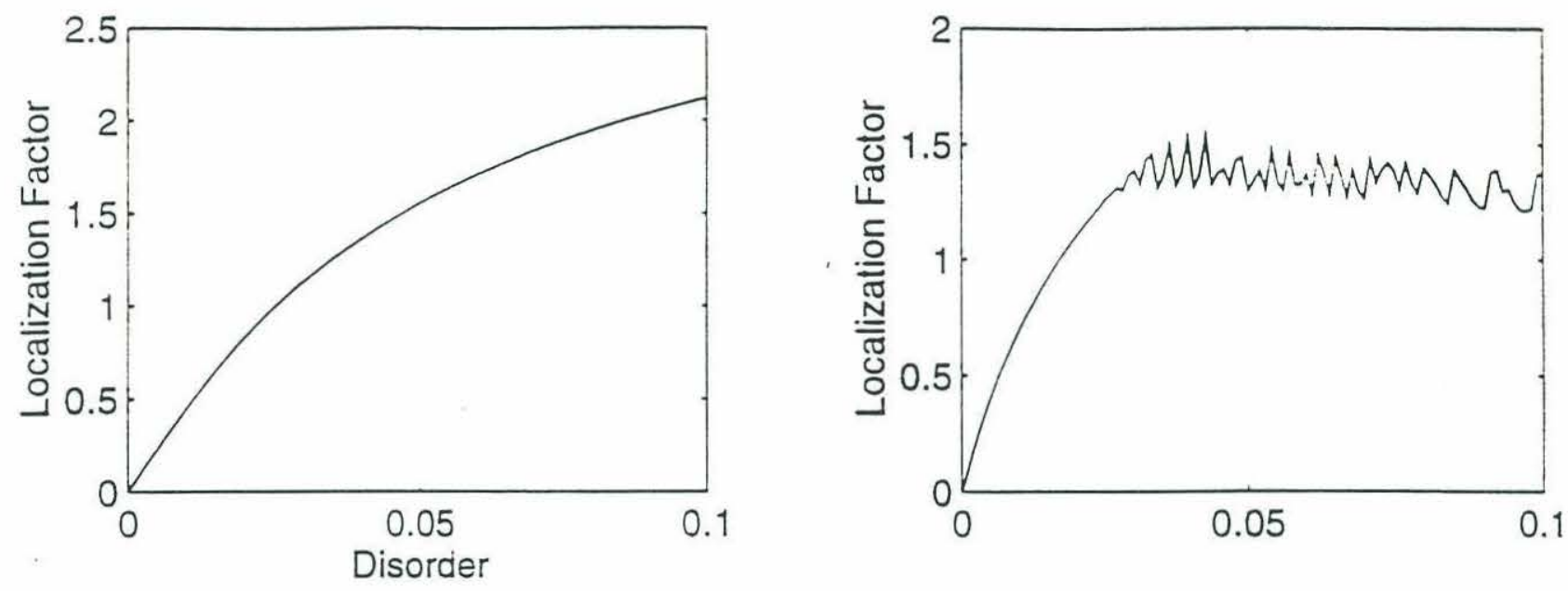

Figure 4-21: Localization factor as a function of end pendulum disorder for three and thirty pendula system

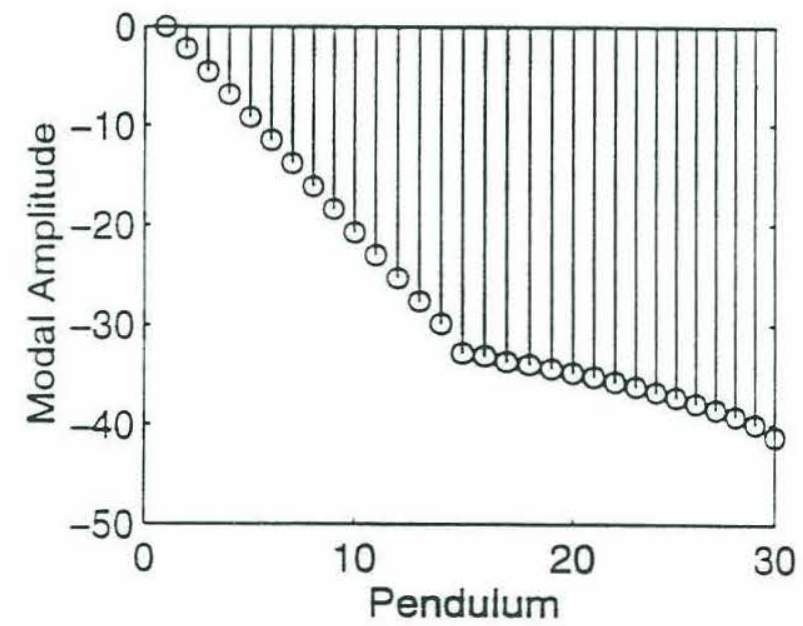

Figure 4-22: Log of absolute amplitude of mode shape of thirty pendula șstem 
insidious effects of machine accuracy have resulted in the mode shape not having a clean exponential profile. The numerical noise would result in the localization factor fluctuating in a discontinuous manner when plotted as a function of disorder. All derivatives are evidently discontinuous and hence there would exist problems in finding an optimal solution. The reason is easy to understand. Consider a localized mode with localization factor $\gamma$. Consider the mode near its peak. On either side the amplitude decays as $e^{-\gamma n}$ where $\mathrm{n}$ is the number of oscillators. If $\gamma=2$, for $\mathrm{n}=3$, we should see a decay of $e^{-6}$ away from the peak. If $n=30$, we see a decay of $e^{-60}$ away from the peak. Clearly, the decay would result in the modal amplitude being below resolution and the effects of roundoff would alter the computed localization factor since part of the mode shape would have the well defined exponential decay and part would have an amplitude which is below machine tolerance. During calculation of the localization factor, we usually only retain the part of the mode with the well defined decay for computing the localization factor. This became important for larger systems of coupled pendula.

We also use our knowledge of the geometry of the system to search for special configurations using our knowledge of the geometry. We have seen in Chapter 3 how in general if we clumped groups of pendula together to have the same disorder, they would be associated with eigenvalue coalescences of that size. We now look at a system of eight pendula and consider the pendula in groups of four and two. Thus for the first case we see the optimal solutions for a system of eight pendula in two groups of four (we are thus looking for solutions close to four root coalescence). These are shown in figure 4-23. In figure 4-24, we examine the optimal solutions for the case where we have four groups of two root coalescences. Again note that some of the modes appear to be subsets of the modes for the previous case. We have specified a minimum localization factor of $\gamma=1.0$ in these cases. For the case of the solution close to four root coalescence, we see the existence of mode shapes which have two sets of modes. Each of these sets of modes appear to be very similar to those associated with those of a system of four identical pendula. The first four modes are thus localized about this set of four pendula. The second set of modes are localized 


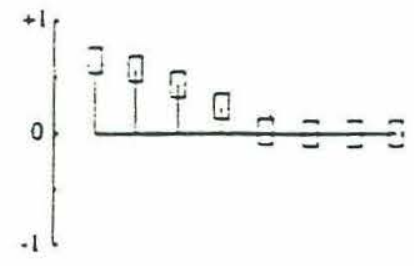

(a)

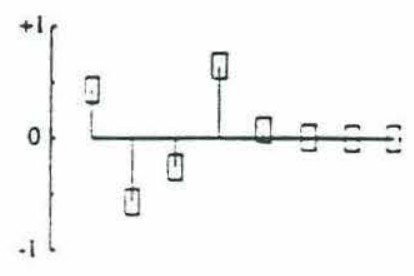

(c)

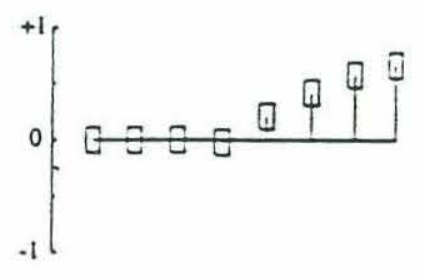

(e)



(a)

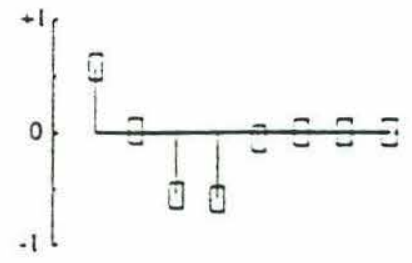

(b)

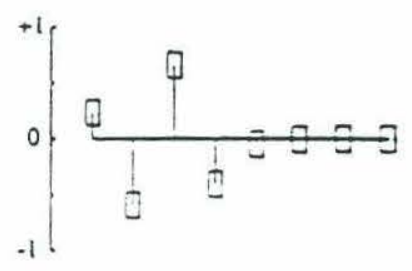

(d)



(I)



(h)

Figure 4-23: An Optimal Solution for the Eight Pendula Problem : Two groups of
four root coalescences 


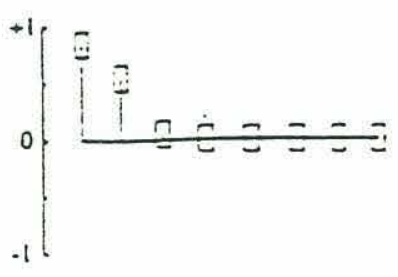

(a)

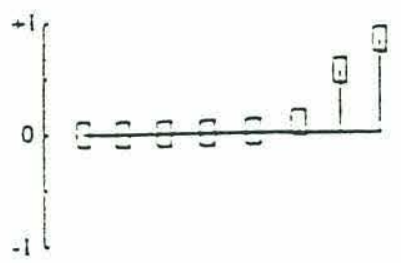

(c)

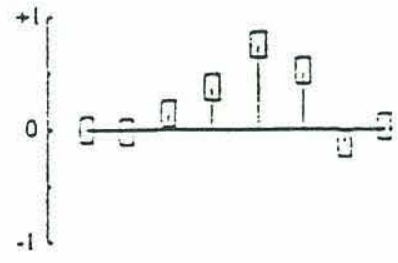

(e)

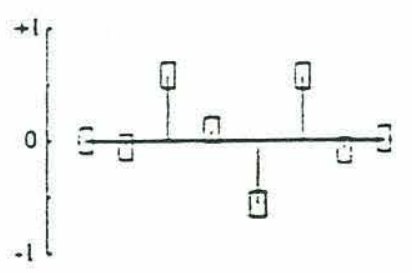

(5)



(b)

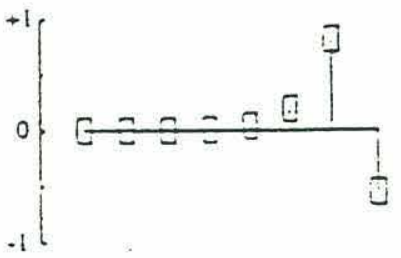

(d)

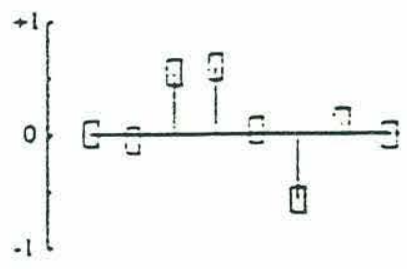

(I)

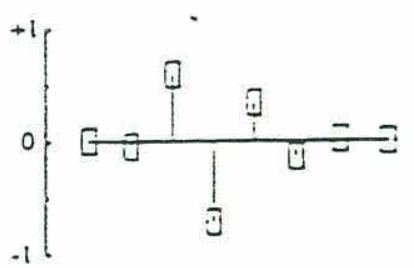

(a)

Figure 4-24: An Optimal Solution for the Eight Pendula Problem : Four groups of two root coalescences 
about the other four pendula.

The second case involving four groups of two pendula is also very educative. If we consider only those oscillators of a mode which have significant amplitude, we now have modes similar to those of a set of two identical pendula. Four modes are localized about a group of two pendula at a time. The modes which are not localized about two pendula have the lowest value for the localization factor.

\subsection{Conclusions}

We have used nonlinear optimization techniques in this chapter to design structures which would minimize vibration transmission. Two problems were studied. The first problem was that of introducing disorder into the systems in such a fashion so as to ensure all modes were localized to some minimum level. The disorder would be selected so as to minimize the sum of squares of the disorder. The second problem studied was that of maximizing the localization in the system so that the sum of the squares of the disorder would be some specified amount. We examined the spatial distribution of the disorder in the first problem and found that the position of the branch point was the exclusive factor in determining this distribution. The optima were along the line of maximum distance from the two root coalescence lines. The two root coalescence lines also divided the space into the convergence basins for each optimum. In order to determine the global minimum, we provided a search procedure which utilized our knowledge of the distribution of optima to systematically track down all optima. We studied the spatial distribution of optima for the second objective function in reference to the branch point distribution and again found the distribution of minima to be along the line of maximum distance from the two root coalescence lines. We found the number of optima and the computational effort grew exponentially with the the number of disorder parameters. Hence for large problems (eighteen to twenty disorder parameters), computational effort would be too large to find the global optimum. However for smaller problems(two to ten problems), a statistical analysis was carried out to prove that sampling as few as $10 \%$ of the 
optima would provide us with an estimate within $2 \%$ of the global optimum. We also examined a few larger systems of pendula and obtained locally optimal solutions and determined a few special optimal configurations. 


\section{Chapter 5}

\section{Mode Localization as a passive vibration isolation device in a real-world structure}

\subsection{Introduction}

We now examine the applications of localization for passive vibration isolation in a real world structure. We consider an oceanographic mooring system which has submerged buoys at regular intervals. The buoy is a symmetric structure and hence the wave induced excitation due to pressure loading would be vertical and the vibrations which would be excited would be inline, elastic excitations in the cable. We assume there will be no surge.

We consider two types of mooring conditions. The first is the case where we have a fixed end condition at the lower end and the second is the case where we have a free end condition at the lower end. The first corresponds to a fixed mooring and the second to a drifting buoy-cable system. 


\subsection{Work done in this chapter}

We write the equtions of motion for the simplified model of the mooring system. We demonstrate that all harmonic modes of the structure can be localized by adding disorder. We demonstrate that localization of response can be obtained for typical sea states only for deep waters of $1000-4000 \mathrm{~m}$. It does not work for shallow waters(40$50 \mathrm{~m})$. We demonstrate that different boundary conditions(towed and moored) do not affect the degree of localization of response.

\subsection{Equations of Motion}

Consider the free body diagrams of sections of the cable shown in figure 5-2. The equations of motion for the stretch of cable between masses are

$$
\rho A \frac{\partial^{2} u_{i}}{\partial t^{2}}=E A \frac{\partial^{2} u_{i}}{\partial s^{2}}
$$

where $s$ denotes the distance along the cable, $t$ denotes time, $u_{i}$ denotes the inline displacement of the $i$ th segment, E denotes the Youngs Modulus, A denotes the cross sectional area of the cable.

The equations of motion for the intermediate masses is

$$
m_{j} \frac{\partial^{2} u_{i}}{\partial t^{2}}+b_{j}\left(\frac{\partial u_{i}}{\partial t}\right)=E A\left(\frac{\partial u_{i+1}}{\partial s}-\frac{\partial u_{i}}{\partial s}\right)
$$

where the mass $m_{j}$ refers to the virtual mass of the $j$ th buoy and $b_{j}$ is the damping associated with the buoy. Note $1 \leq j \leq N-1$ and $1 \leq i \leq N$ with $j=i$. During the analysis, we will assume that the primary source of drag is separation drag and will use standard drag coefficients. This is reasonable because, the dimensions of the subsurface buoy are such that viscous effects are insignificant compared to the separation induced drag.

The boundary conditions are given by

$$
U_{1}(0)=0
$$




$$
U_{n}(n L)(0)=0
$$

For boundary condition 2, we modify the zero displacement condition at the lower end to result in

$$
\frac{\partial U_{n}(n L)}{\partial x}=0
$$

This is the condition for zero force at the lower end. Thus the boundary condition matrix would be slightly altered for this case.

The equations given above can be solved using the transfer matrix formulation described in Pestel and Leckie [26].

\subsection{Modes of Vibration of Periodic and Disor- dered Structure}

We consider an oceanographic mooring system as shown in figure 5-1. Free body diagrams of the mass and cable are shown in figure 5-2. Note that there is an infinite set of natural modes of the system, but we will restrict our attention to the lowest set of modes.

We consider a system with four masses on it. The disorder introduced into the system is shown in table 5-1. They are placed $200 \mathrm{~m}$ apart as shown in table 5-2. The total length of the mooring is $1000 \mathrm{~m}$. The disorder shown in table 5-1 was picked from a uniform distribution spread between -50 and $+50 \mathrm{~m}$ (i.e. $\pm 25 \%$ ). The added mass for a sphere is obtained from Blevins ([4]).

The first four modes of the periodic system are shown in figure 5-3. These modes have wavelengths of the order of the entire structure and form the fundamental set of modes. Others have half wavelengths of the order of the length of the distance between the masses. These form the first harmonic set of modes which are shown in figure 5-4. Still others have half wavelengths of the order of the half the distance between adjacent masses. These form the second harmonic set of modes and are 


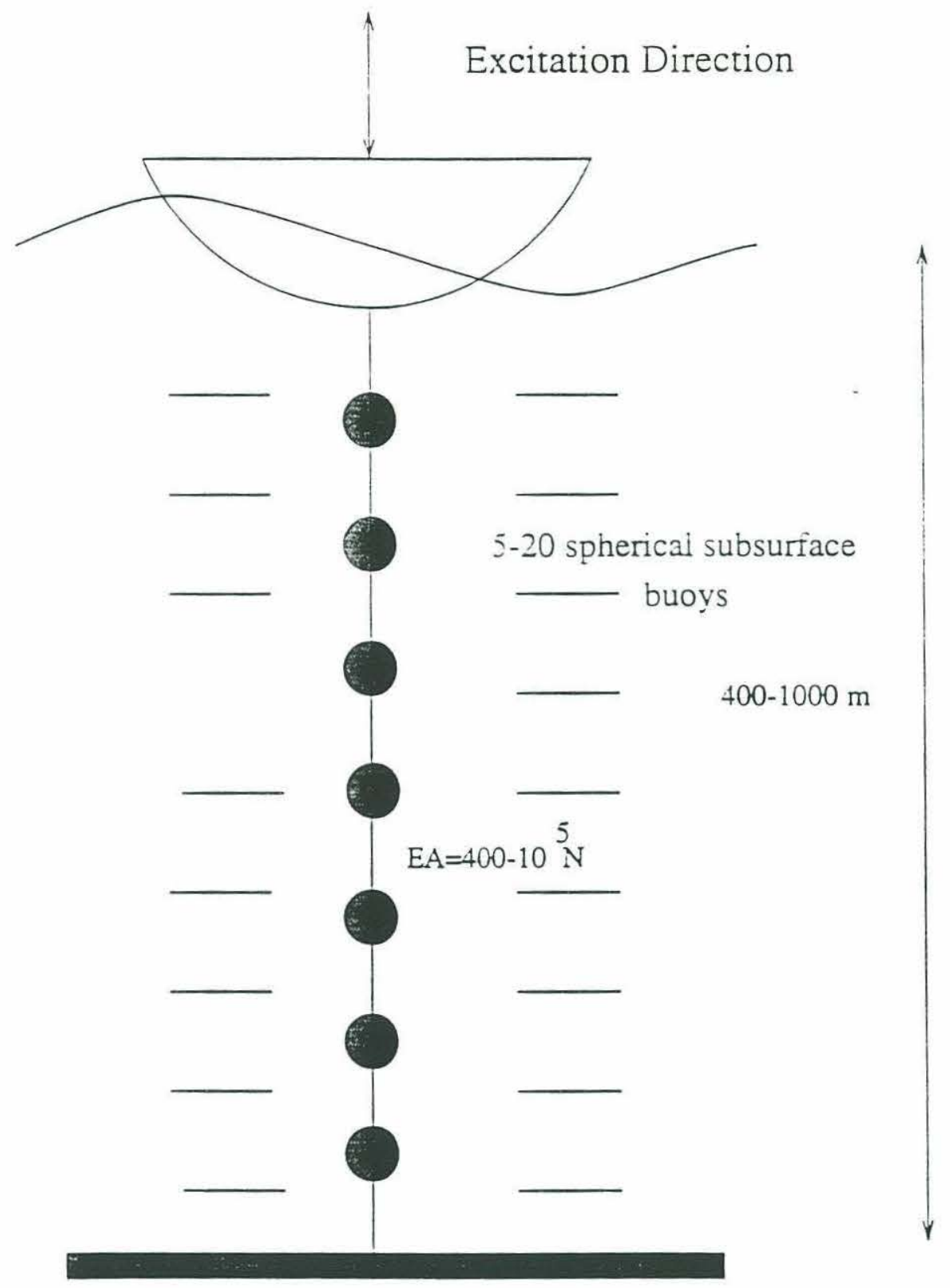

\section{FIGURE NOT DRAWN TO SCALE}

Figure 5-1: Schematic Diagram of Oceanographic Mooring 
(a) Free Body Diagram for Cabie Element

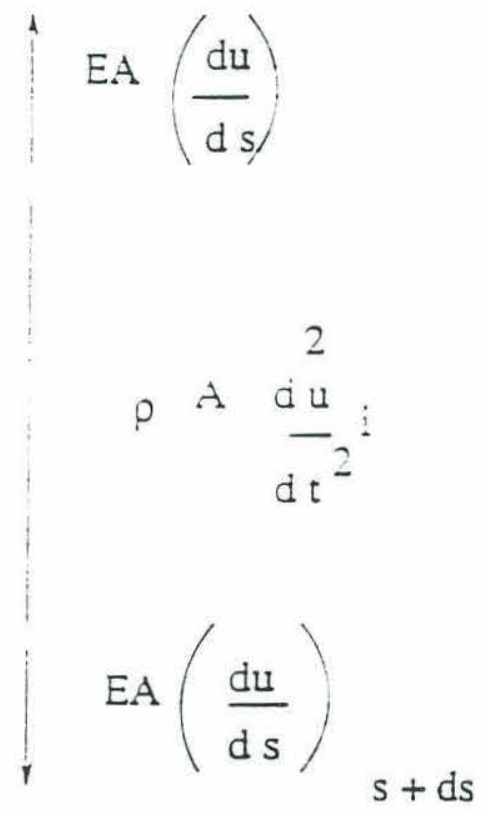

(b) Free Body Diagram of Buoy

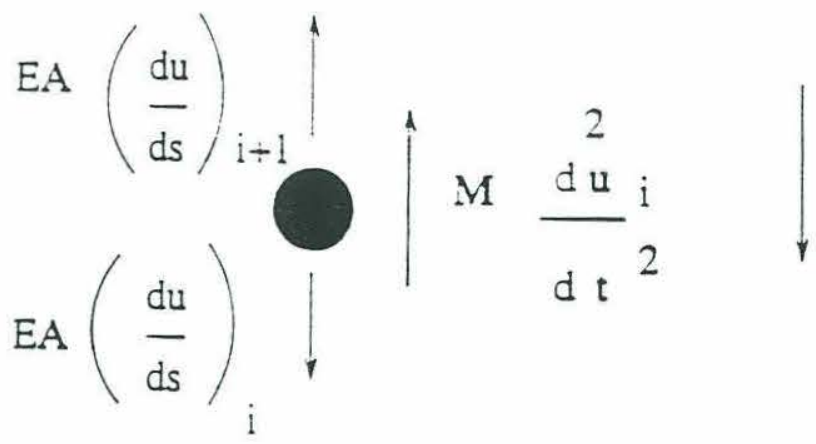

Figure 5-2: Free Body Diagram of Parts of Mooring. $u_{i}$ : Displacement of $i$ th segment. $M$ : Virtual Mass of buoy. $s:$ Coordinate along cable. $D:$ Drag on buoy. $E$ : Youngs modulus of the cable. $A$ : Area of cable. $t$ : time 


\begin{tabular}{||c|c||}
\hline \hline Mass & Disorder \\
\hline \hline 1 & $-29,9849$ \\
2 & 43.3032 \\
3 & -3.4485 \\
4 & .7147 \\
\hline \hline
\end{tabular}

Table 5.1: Disorder

\begin{tabular}{||c|c||}
\hline \hline Cable & Mass \\
\hline \hline$E A=600 \mathrm{~N}$ & $\mathrm{M}=200 \mathrm{~kg}$ (sphere) \\
$\rho=1070 \mathrm{~kg} / \mathrm{m}^{3}$ & $\mathrm{~d}=.669 \mathrm{~m}$ (sphere) \\
$d=.0252 \mathrm{~m}$ & \\
$n L=1000 \mathrm{~m}$ & \\
\hline \hline
\end{tabular}

Table 5.2: Column 1: Cable Parameters, Column2: Mass Parameters 

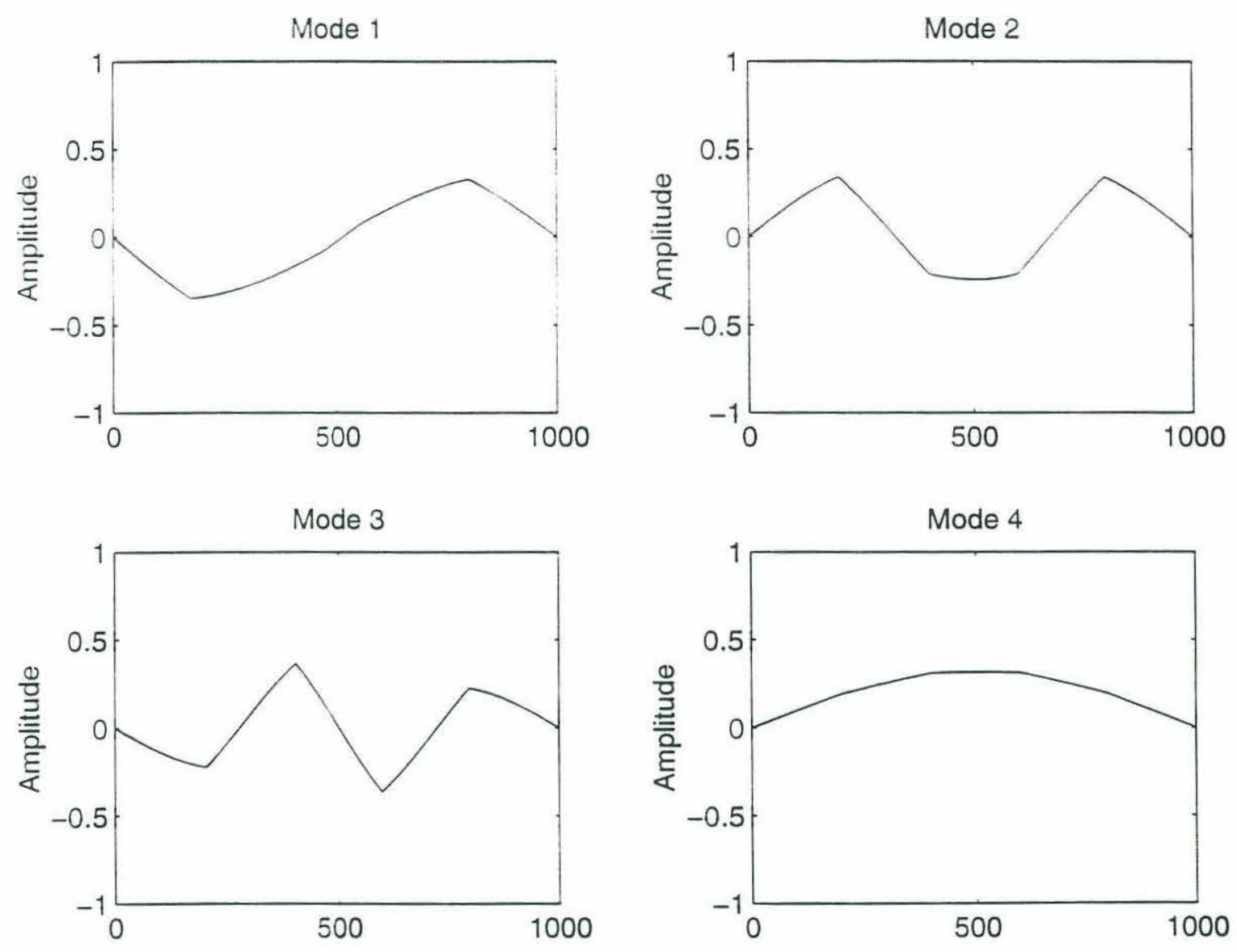

Figure 5-3: Fundamental Set of Modes for Periodic Structure 



Figure 5-4: First Harmonic Set of Modes for Periodic Structure 

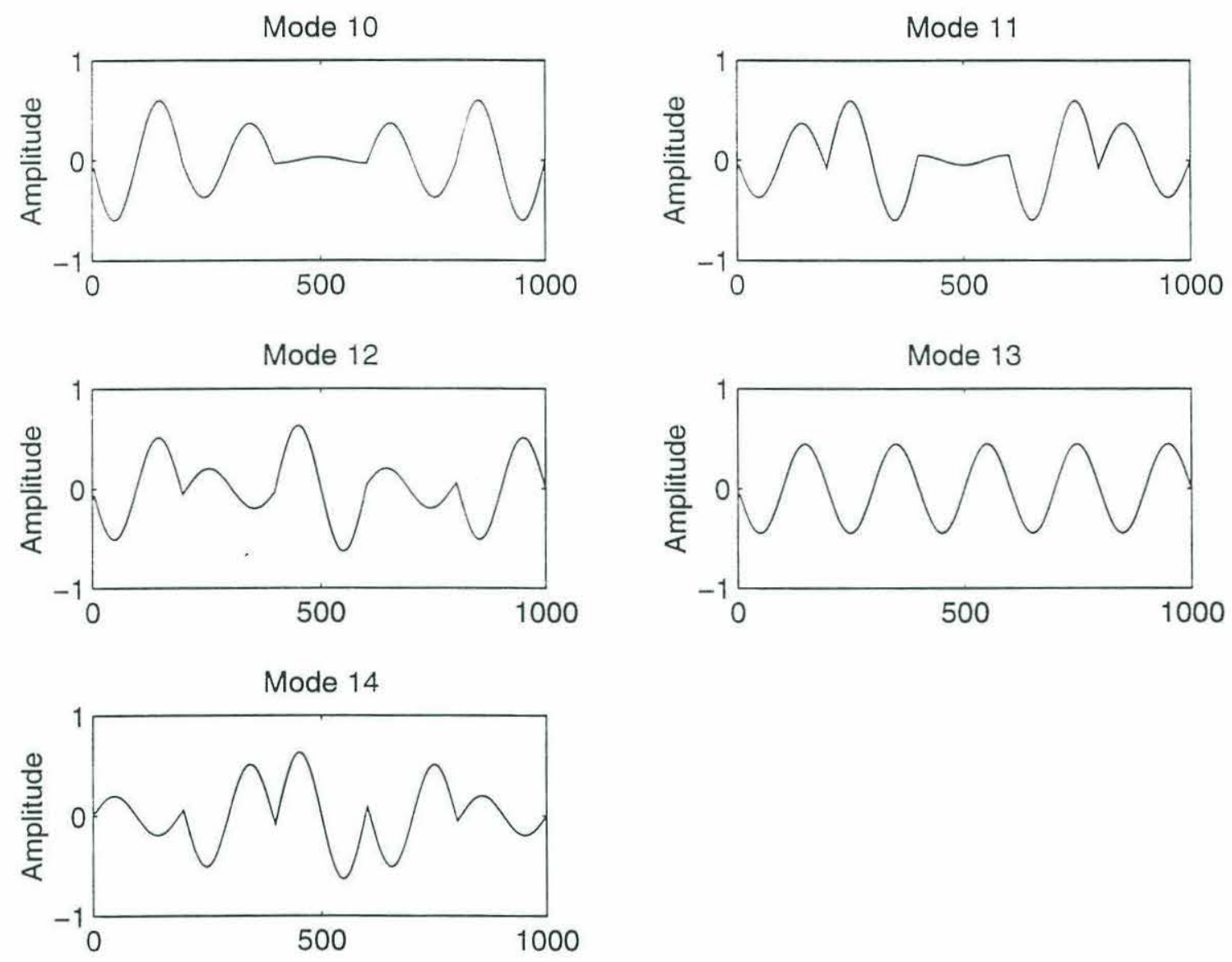

Figure 5-5: Second Harmonic Set of Modes for Periodic Structure 
shown in figure 5-5. Note that although we are examining in plane displacement, we have plotted the displacement in a transverse fashion. This is only for ease of viewing. The modes of the disordered structure for configuration (a) are shown in figure $5-6,5-7$ and 5-8. Note that the fundamental set of modes shown in figure 5-6 are not localized. But all the higher groups of modes as shown in figure 5-7 and 5-8 are localized. Thus localization is not useful for localizing low frequency vibration. It is useful for localizing higher frequency oscillations. Essentially, the reason is that in situations where the modes can be localized, the stretch of cable between the masses can be viewed as an oscillator with the mass serving as a decoupling element. In the limit of the mass tending to infinity, we recover the degenerate situation corresponding to a set of decoupled pendula and for the other situation where the mass tends to zero, we recover the asymptotic case corresponding to a system of rigidly coupled pendula.

Localization can thus be used as a passive vibration isolation device only in a restricted set of frequencies. During applications, we have to ensure that the distribution of the passband natural frequencies has a special distribution for the vibration isolation to be effective.

However for oceanographic structures in relatively deeper waters, as we will see, this is precisely the distribution of the passband natural frequencies and can be exploited to design a passive vibration isolation device. We note that for the effective utilization of mode localization as a passive vibration isolation device, we need to ensure that the fundamental set of modes which are nonlocalizable have to be at the lower end of the sea-spectrum where there is no energy while the localizable modes may be permitted to remain in the region of the sea spectrum where there is significant energy. We use a Pierson-Moskowitz Spectrum to represent the sea-state assuming a fully developed sea-state (fetch-independent) with a modal frequency at $.5 \mathrm{rad} / \mathrm{s}$. The Pierson-Moskowitz Spectrum is generated using

$$
S(\omega)=8.110^{-3} \frac{g^{2}}{\omega^{5}} e^{-\frac{.74\left(\omega^{4}\right)^{4}}{\omega^{4}}}
$$



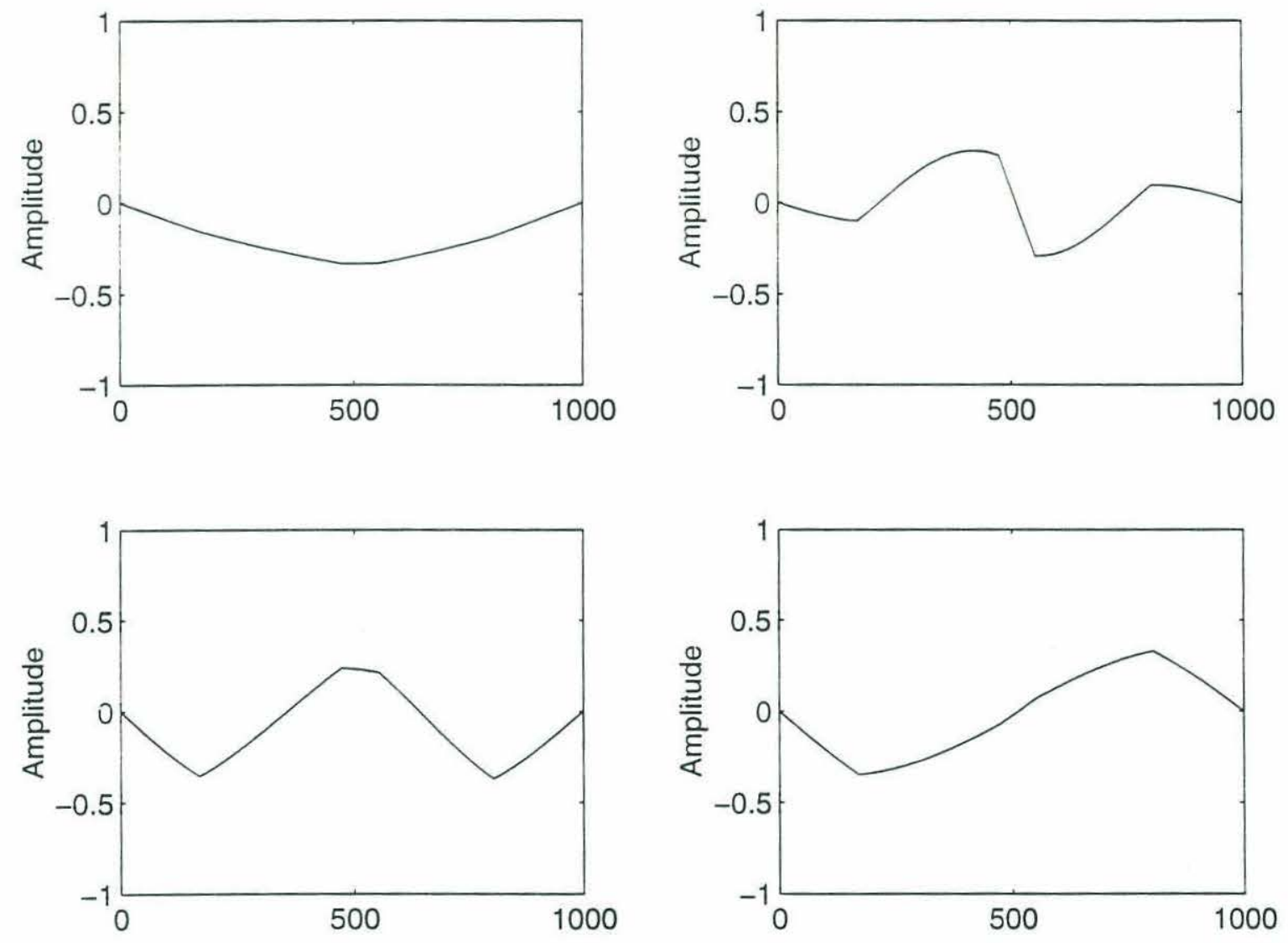

Figure 5-6: Fundamental Set of Modes for Disordered Structure 



Figure 5-7: First Harmonic Set of Modes for Disordered Structure 

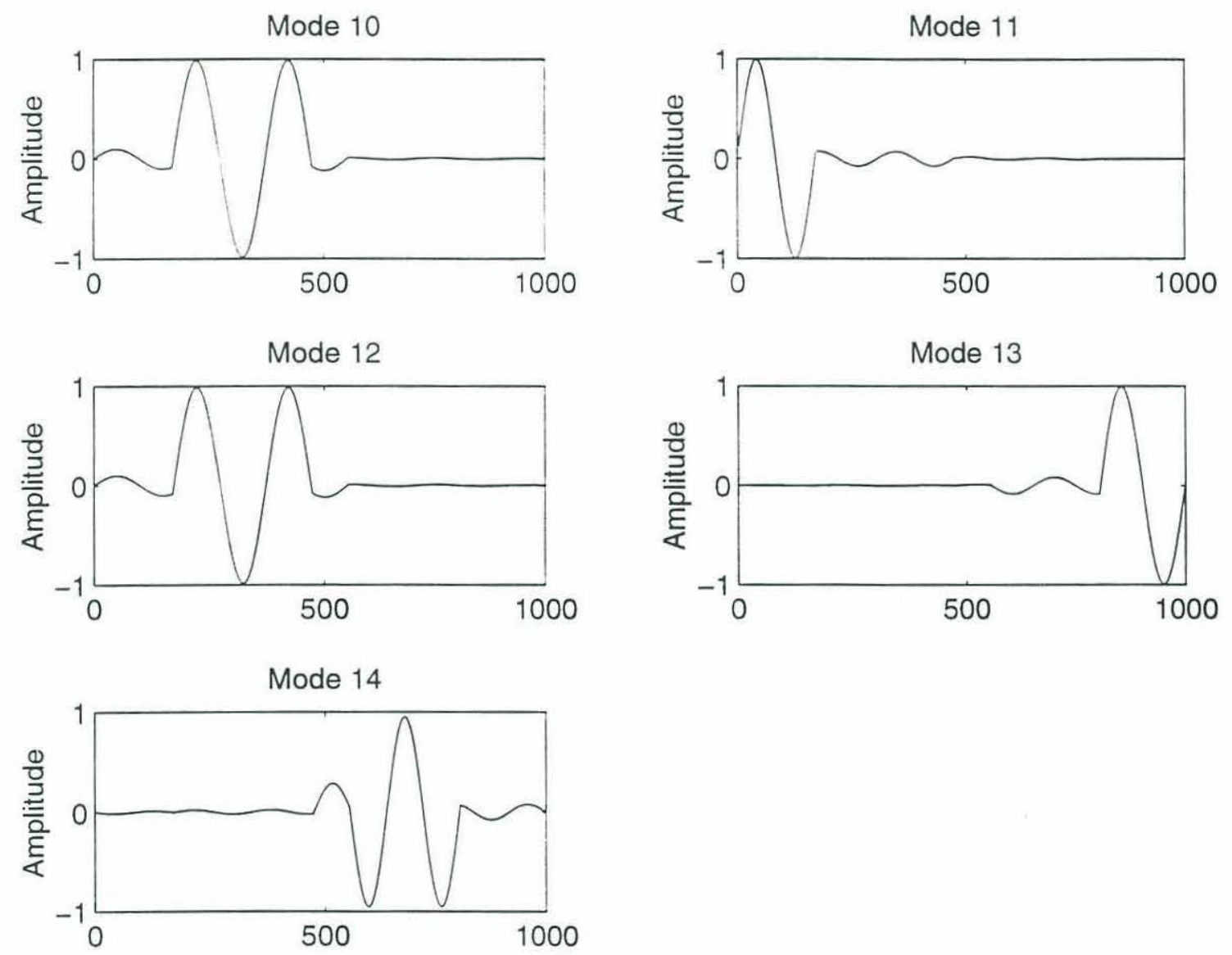

Figure 5-8: Second Harmonic Set of Modes for Disordered Structure 
where $\omega_{m}$ is the modal frequency and $S(\omega)$ is the spectrum. This is shown in figure 5-9.

We summarize the design constraints

(a) The lowest passband corresponds to modes whose half wavelengths are of the order of the whole structure and cannot be localized.

(b) The lowest passband whose modes can be localized is that for which the half wavelength of the center frequency is of the order of the distance between the masses.

(c) The sea-spectrum is such that the wave zone is between 0.4-1.2 rad/s. So the lowest passband (nonlocalizable) should be positioned at frequencies below the wave zone. The higher passband (localizable) can be positioned at the wave zone or higher.

We can do a preliminary design feasibility plot to determine the parameter ranges in which we could have a feasible design given these constraints. We note the if we desire half wavelength of the order of the distance between the masses or smaller,

$$
\omega=\sqrt{\frac{E A}{\rho A}} \frac{2 \pi}{L}
$$

Here $L$ is the distance between the masses. We require $\omega$ to be between $0.4 \mathrm{rad} / \mathrm{s}$ and $1.6 \mathrm{rad} / \mathrm{s}$. $\rho$ is fixed for the cable. So we prepare a plot indicating the variation of $E$ and $L$ with $\omega$. This is shown in figure 5-10. We note that we can use this in shallow water moorings $(40 \mathrm{~m})$ only if we have very low Youngs Modulus Values of $E=10^{4}$ Pascals. With existing technology (we quote Youngs Modulus values from commercial material available from Buoy Technology Inc.), we would need distances between the masses to be $150-200 \mathrm{~m}$. The Youngs Modulus value here was $E=1.2 \times 10^{6}$ Pascals. So we shall focus on deep water applications.

We now consider the response of the structure to typical sea-spectra as shown in figure 5-9. We consider a structure with 20 segments of cable and 19 masses between them. The masses are separated by lengths of $200 \mathrm{~m}$. The details of the cable and mass are given in table 5-3. The total length of the structure was $4000 \mathrm{~m}$.

The damping constant $\mathrm{b}$ is estimated using the equation

$$
b=C_{D} \rho A
$$




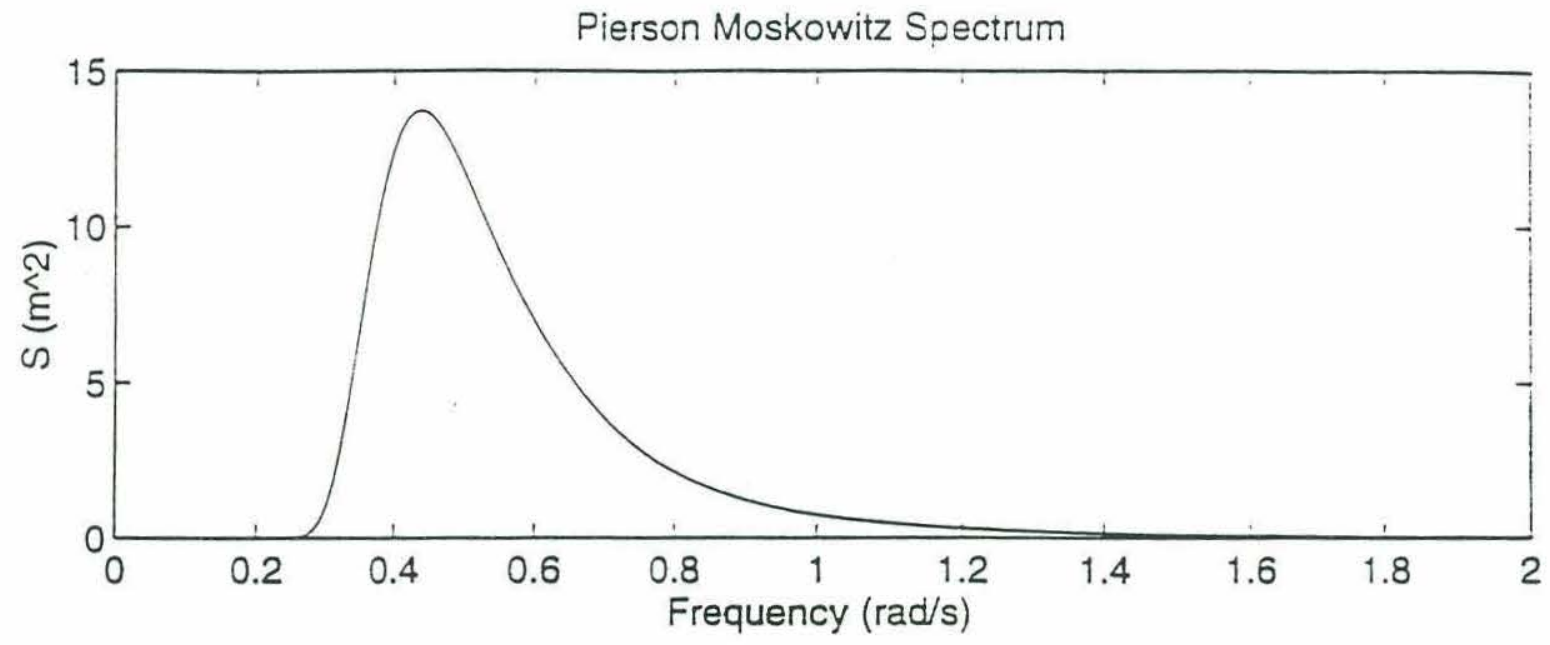

Figure 5-9: Pierson Moskowitz Spectrum 




Figure 5-10: Design Curves for selection of $E$ and the distance between masses 


\begin{tabular}{||c|c||}
\hline \hline Cable & Mass \\
\hline \hline$E=10^{5} \mathrm{~N}$ & $\mathrm{M}=5000 \mathrm{~kg}$ (sphere) \\
$\rho=1070 \mathrm{~kg} / \mathrm{m}^{3}$ & $\mathrm{~d}=1.54 \mathrm{~m}$ (sphere) \\
$d=.3257 \mathrm{~m}$ & \\
$n L=4000 \mathrm{~m}$ & \\
\hline \hline
\end{tabular}

Table 5.3: Column 1: Cable Parameters, Column2: Mass Parameters

The coefficient of drag $C_{D}$ associated with the submerged sphere (This is taken to be .1 from Newman [24]), $\mathrm{V}$ is the velocity associated with the motion of the sphere, and $\mathrm{A}$ is the projected area on the direction of motion of the sphere. This is included in the equations of motion for the submerged sphere.

If $H(\omega)$ is the transfer function of the system and $S(\omega)$ is the sea-spectrum, then the Response Amplitude Operator is

$$
Y(\omega)=|H(\omega)|^{2} S(\omega)
$$

In figure 5-11, we show the transfer function of the mooring for the periodic case. Note the first bay has significantly more amplitude than the other bays because of damping but there is clear evidence of a spatially extended response. In figure 5-12, we show the transfer function of the mooring for the disordered case. The disordered system shows clear evidence of vibration isolation with the lower half of the structure showing almost zero amplitude.

We now examine the responses of the same structure but with the free end condition corresponding to the towed condition. We also ensure that for the disordered structure, the same set of disorder is used to provide comparison.

We now examined the disordered case in figure 5-13. The response is slightly more localized than in figure $5-12$ but not significantly so. Hence we can conclude that changing the boundary conditions did not alter the fundamental nature of the response for the localized case. 
Twenty Bays, Periodic Case

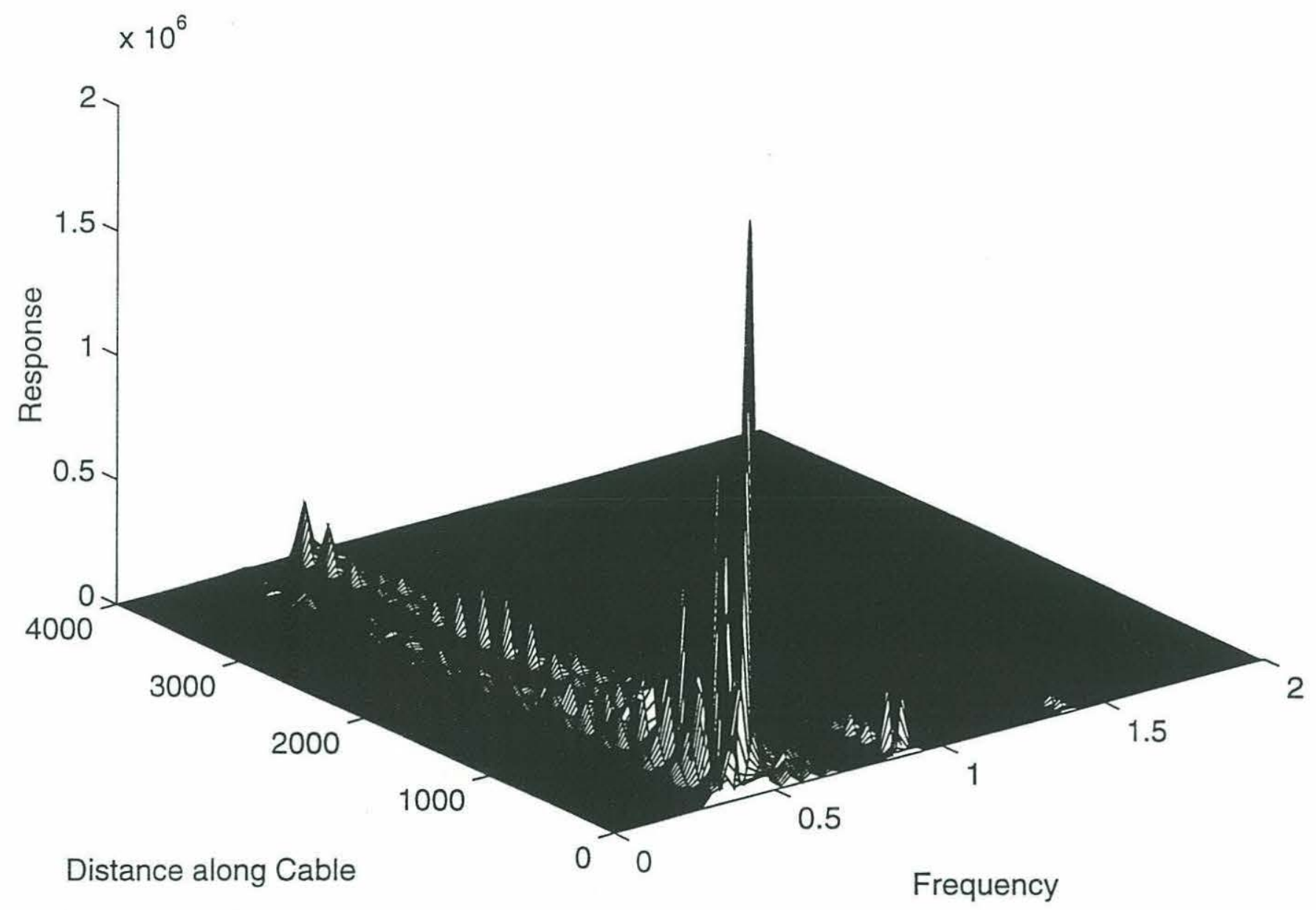

Figure 5-11: Transfer Function for Periodic Case 
Twenty Bays, Disordered Case



Figure 5-12: Transfer Function for Disordered Case 


\subsection{Conclusions}

We demonstrate the application of mode localization to a practical structure (an oceanographic mooring). First. we demonstrate the existence of localization of harmonic modes in this structure. We did not find localization useful as a passive vibration isolation device for shallow water moorings $(40 \mathrm{~m})$ but we found it useful for deep water moorings from 1000-4000 m. We ensure that nonlocalizable (fundamental) modes fall outside the wave zone of the sea spectrum. We found that altering boundary conditions (towed and moored) does not result in any significant change in localization. 
Twenty Bays, Disordered Case, Boundary Condition 2

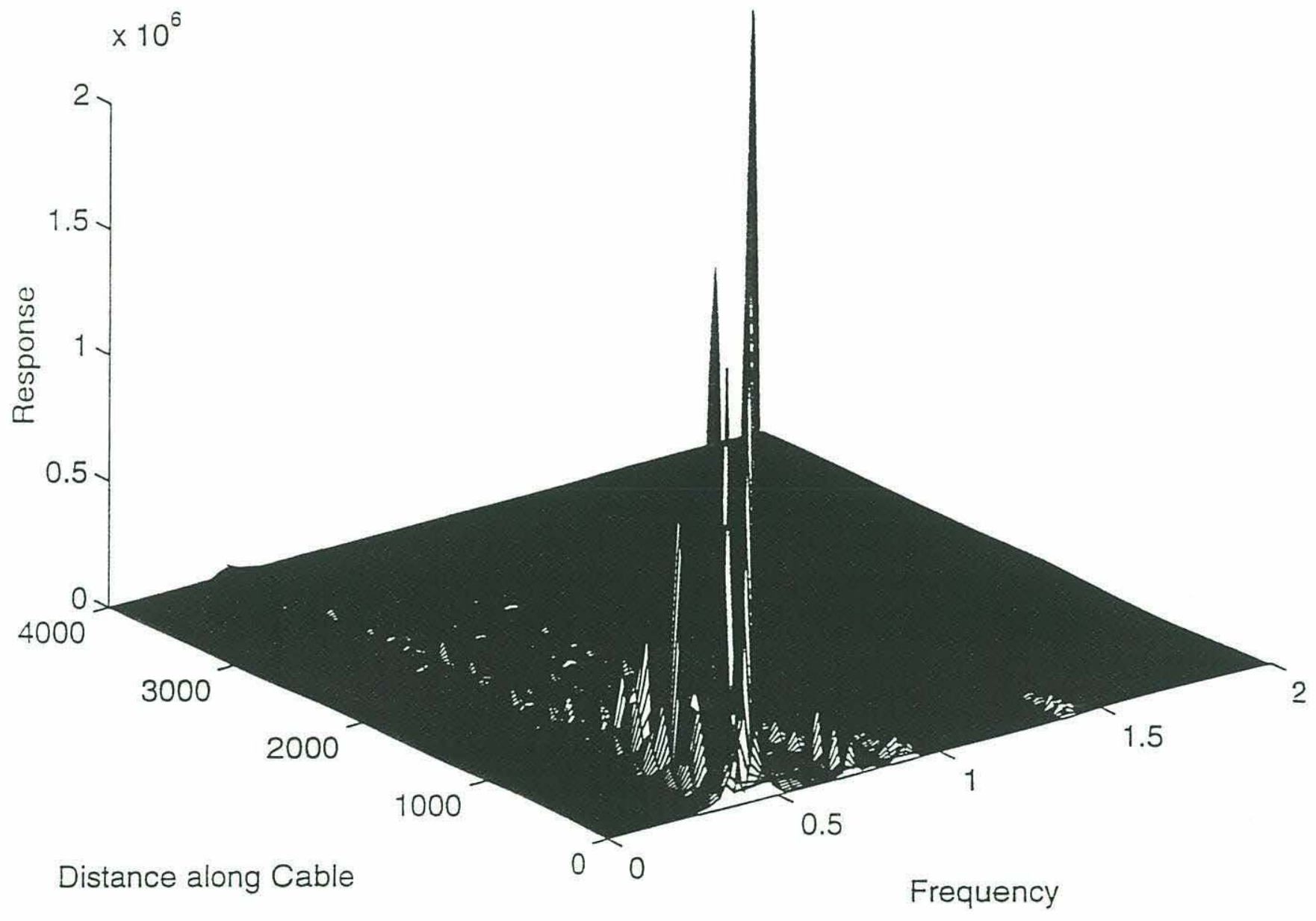

Figure 5-13: Transfer Function for Disordered Case for Boundary Concition 2 


\section{Chapter 6}

\section{Conclusions and}

\section{Recommendations for Future}

\section{Work}

\subsection{Main Features}

We will, in this section, briefly describe the main features of the work in this thesis.

This work has succeeded in filling a few gaps existing in research in the area of localization caused by deterministically introduced disorder.

Perhaps the best way to realize where this work fits in is to understand the chain of research that has occured in the field of localization. The work started with seminal work by Hodges and Woodhouse [16] (See the tree in figure 6-1). Their papers ([17], [19]) resulted in a series of spin-off papers by other authors (Kissel [20], Pierre et al. [27], [29], [28], [31] and Triantafyllou and Triantafyllou [39]). Hodges and Woodhouse [18] also interpreted many of the results of solid-state literature in a form that was meaningful to the structural-dynamics community.

The precise niche that this thesis has carved out for itself is apparent by viewing figure 6-1. The main contribution of this thesis is in providing a framework to understand modal sensitivity and then using that to create a new area of study i.e. optimal 
localization. This has tremendous potential in the area of passive vibration isolation.

\subsection{Summary of Conclusions}

In Chapter 2, we showed that Jordan Block perturbation expansions about branch points in the complex plane are a useful tool to describe modes of a real system of pendula in a state of moderate localization. We also solve the branch point equations to obtain the convergence zones of the various perturbation techniques.

In Chapter 3, we provided numerical confirmation of the fact that modal sensitivity and localization were phenomena associated with the presence of branch points. We examine the effects of parameter combinations which result in the system of pendula being close to branch points of different orders. Sensitivity due to branch points depends on the order of the branch point and the magnitude of the imaginary part. The modes associated with different order branch points have different appearances. The conflicting effects of the two parameters on localization are examined. The existence of optimal directions in the parameter space along which localization is a maximum is also noted.

In Chapter 4, we solve the problem of selecting parameter combinations to ensure all the modes have a certain minimum level of localization. The optimal solutions were found to be at maximum distance from the branch point surfaces and the convergence basins of the optimal solutions were found to be divided by the sectors of space created by the two root coalescence lines. This knowledge was used to install a numerical scheme to track down all the optima. The initial search determined a point within the convergence basin and reasonable close to the final solution. The final solution was found by using a steepest descent method. The number of optima and computational effort was found to grow exponentially with the number of pendula. A statistical analysis was done to show that sampling as few as $10 \%$ of the optima provided a solution within $2 \%$ of the global optimum. However due to the exponential growth of optima and effort with the number of disorder parameters, this result only helps for smaller systems of pendula (approximately two to ten pendula). For larger systems, 


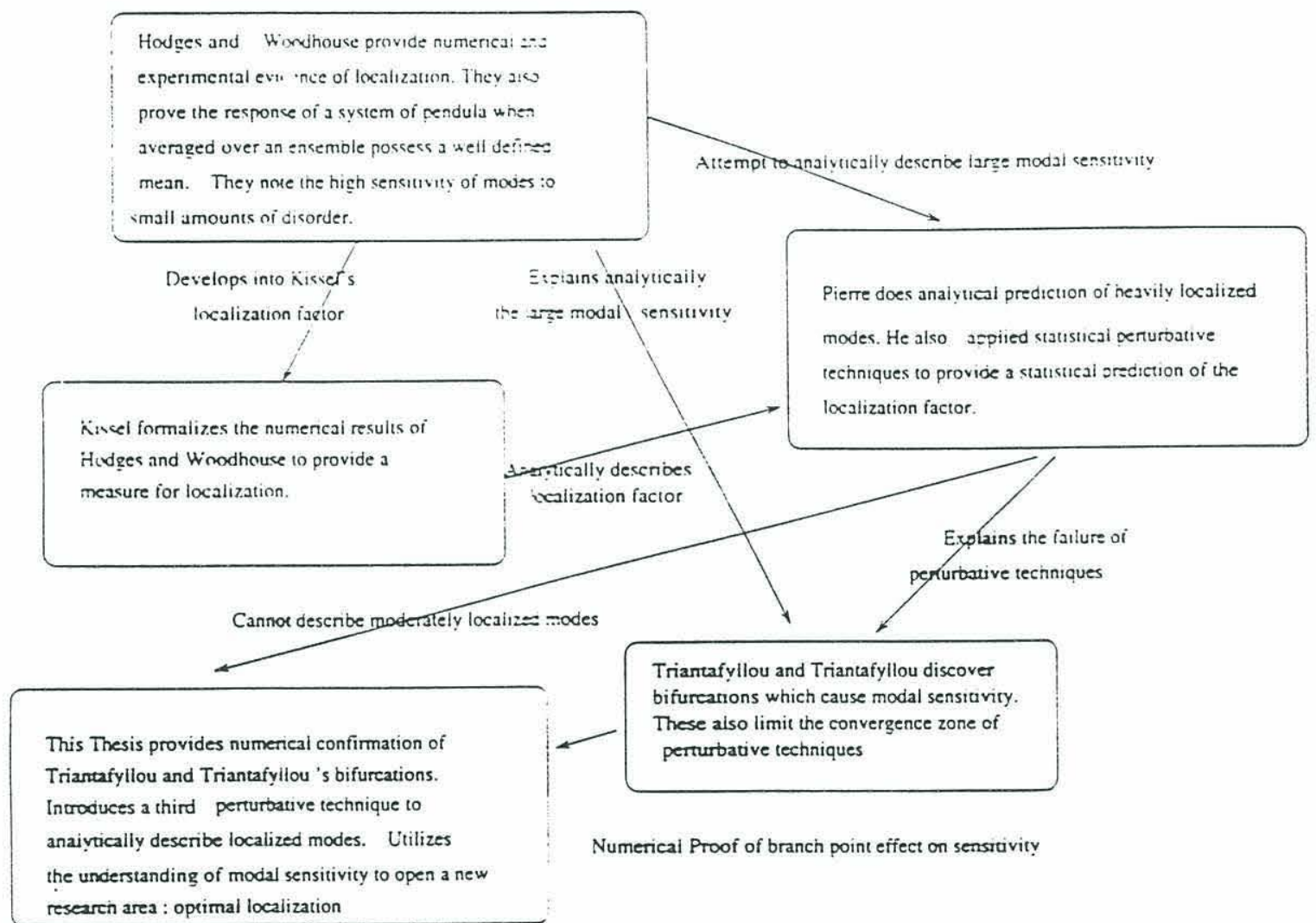

Figure 6-1: Flow of Research in Mode Localization 
the large number of optima makes it impossible to determine the global optimum in any reasonable time. This is a failing in the research.

Applications of localization as a passive vibration isolation device were studied. The structure studied was an oceanographic mooring with regularly spaced subsurface buoys and the vibrations of concern were the inline, elastic oscillations. It was found useful for localization of the harmonics. It was found useful in deep waters of depths from $1000 m-4000 m$. Moored and towed boundary condition were examined. It was found that the alteration of boundary conditions resulted in the towed condition having similar localization.

\subsection{Future Work}

Future Work would involve the following three main areas

(a) Determining the branch point surface for a two dimensional system. Comparing how these surfaces with those for one dimensional surfaces.

(b) Determining the bifurcation equations for a continuous system. How do these branch point surfaces look for such a system? Even the continuous system we looked at in Chapter 5 had a discrete aspect in the sense that the mass was a point mass. How would this system link up with the WKB problems studied earlier (Luongo [23])

(c) Determining methods for applying these optimization techniques to continuous systems. In present form, these methods are only applicable to discrete sytems like the system of coupled pendula or spring-mass systems.

(d) Suitable methods to estimate the global optimum of large systems of coupled pendula.

This thesis is only a small step in understanding localization. We have mostly focused on linear dynamics. There is of course considerable research to be done in the area of response localization in coupled oscillators with nonlinear dynamics. A good start has been made by Tjavaras [38] in examining coupled nonlinear oscillators in one dimension. However nonlinear dynamics and localization for two dimensional coupled oscillators is still an unexplored area. 


\section{Appendix A}

\section{Definition of Modal Sensitivity and Localization Factor}

\section{A.1 Definitions of Modal Sensitivity Parameter and Localization Factor}

Consider a dynamic system dependent on only one parameter $\epsilon$. We seek to study the sensitivity of the mode shapes to the disorder parameter $\epsilon$. So we introduce the modal sensitivity parameter

$$
Q(q, \epsilon)=\lim _{\epsilon^{\prime} \rightarrow 0} \frac{\left|q\left(\epsilon+\epsilon^{\prime}\right)-q(\epsilon)\right|}{\epsilon^{\prime}}
$$

This is a classical definition of a derivative with the vertical lines denoting the Euclidean norm in the $n$ dimensional space spanned by the vector. We will use this definition of the derivative to describe the modal sensitivity. In general for a system of pendula, where we have $n$ different disorder parameters $\epsilon_{i}$, we would have partial modal sensitivity parameters (PMSP). The total derivative for the $i$ th mode would then be expressed as follows :

$$
Q\left(q_{i}\right)=\sum_{j=1}^{j=n} Q\left(q_{i}, \epsilon_{j}\right)^{2}
$$


We also define the following measure of localization. Consider the mode shape given by $q_{i}$. We seek to provide a quantitative measure of the localization in the system. Anderson's original theorems on localization predicted an exponential decay in the mode shape especially in the limit of the number of substructures tending to infinity. This was used as the basis for definitions of measures of localization. Most of these used the exponential decay constant associated with the mode shape to provide a measure of the extent to which the mode shapes were localized. They were used in the context of randomly introduced disorder. However, since we are studying localization caused by deterministically introduced disorder, we will use the following measure of localization. Consider the mode shape $q_{i}(j)$ where $i$ denoted the $i$ th mode and $j$ denotes the amplitude at the $j$ th oscillator. Evaluate $y_{i}(j)=\log \left[q_{i}(j)\right]$. Fit a simple regression to the curve. Determine the slope $\gamma$. This is the localization factor.

In figure A-1, we provide an example where the modes of the system are moderately localized and there is a need to determine a logarithmic fit to determine the constant of exponential decay. In figure A-2, we provide an example where the modes are heavily localized and there exists a well defined exponential decay.

There is an obvious connection between localization and modal sensitivity. The modes for any combination of disorder are essentially an integral of the modal sensitivity over the parameter space. The localization factor is the exponential fit associated with the mode shapes.

Thus zones where the modal sensitivity is high also correspond to zones where the localization factor changes very rapidly. In general, the modal sensitivity contours must run parallel to the localization factor contours because of the relation between the modal sensitivity and the localization factor. 




(a) : Mode



(b) : Mode

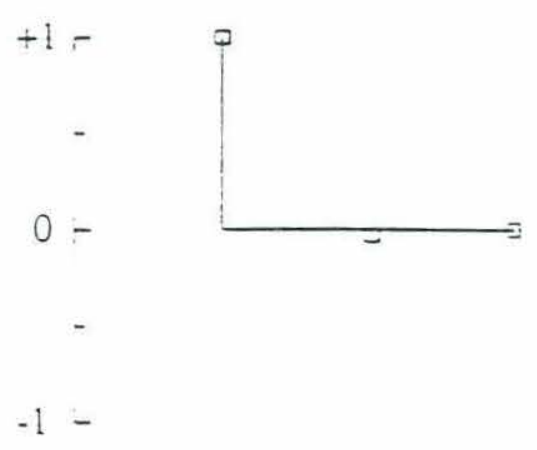

(c) : Mode

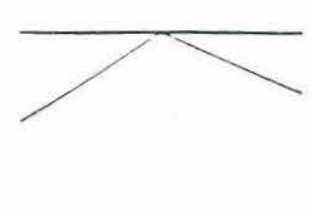

Log Plor


Log Plot

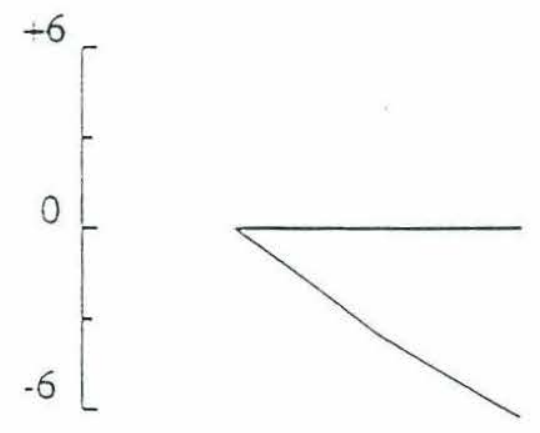

Log Plot

Figure A-1: Example where the exponential fit is good. $\epsilon_{1}=.1, \epsilon_{2}=-.15$ 


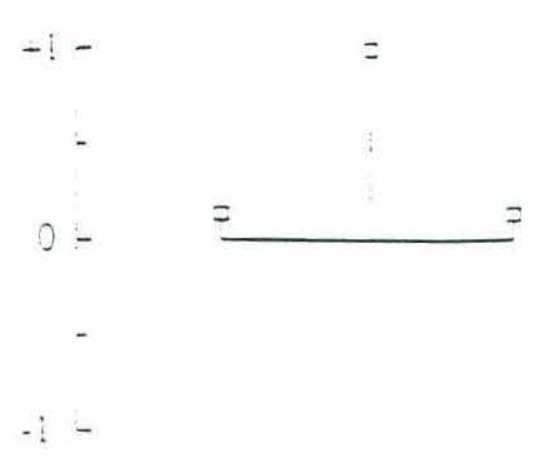

(2): vivae



(b) : Mode

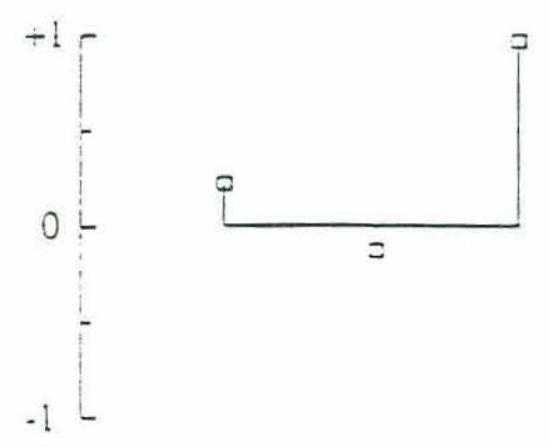

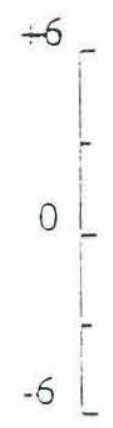

Log Pioc

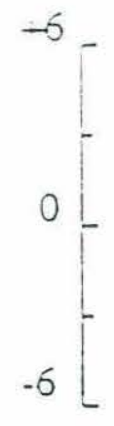

Log Plot

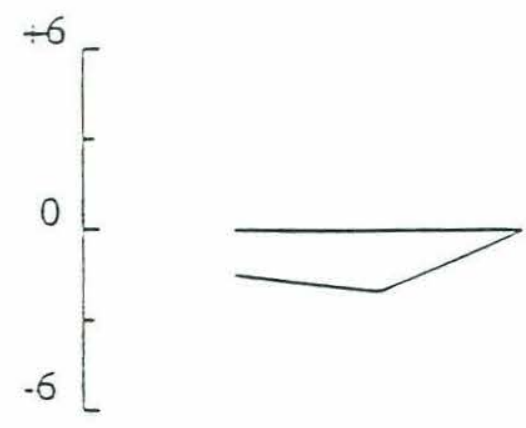

Figure A-2: Example where the exponential fit is poor. $\epsilon_{1}=.005, \epsilon_{2}=.1$ 


\section{Appendix B}

\section{Solutions to Jordan Block Size Three Perturbation}

The perturbation problem for the Jordan block of size three is derived in this appendix. The perturbation series about the Jordan block of size three is written as follows :

$\left[K_{0}+\delta K+\delta^{2} K\right]\left\{x_{01}+\delta^{\frac{1}{3}} x+\delta^{\frac{2}{3}} x+\delta x+\ldots\right\}=\left(\lambda_{0}+\delta^{\frac{1}{3}} \lambda+\delta^{\frac{2}{3}} \lambda+\delta \lambda+\ldots\right)\left\{x_{01}+\delta^{\frac{1}{3}} x+\delta^{\frac{2}{3}} x+\ldots\right\}$

We have expanded the eigenvector and eigenvalue in a series in one-third powers of the disorder.

$$
\begin{gathered}
\boldsymbol{O}(\mathbf{1}) \\
{\left[K_{0}-\lambda_{0} I\right] x_{01}=0} \\
\boldsymbol{O}\left(\boldsymbol{\epsilon}^{\frac{1}{3}}\right) \\
\boldsymbol{O}\left(\boldsymbol{\epsilon}^{\frac{2}{3}}\right) \quad\left[K_{0}-\lambda_{0} I\right]\left\{\delta^{\frac{1}{3}} x\right\}=\delta^{\frac{1}{3}} \lambda\left\{x_{01}\right\} \\
\\
{\left[K_{0}-\lambda_{0} I\right]\left\{\delta^{\frac{2}{3}} x\right\}=\left(\delta^{\frac{1}{3}} \lambda\right)\left\{\delta^{\frac{1}{3}} x\right\}+\left(\delta^{\frac{2}{3}} \lambda\right)\left\{x_{01}\right\}}
\end{gathered}
$$


$O(\epsilon)$

$\left[K_{0}-\lambda_{0} I\right]\{\delta x\}=-[\delta K-(\delta \lambda) I]\{x\}+\left(\delta^{\frac{1}{3}} \lambda\right)\left\{\delta^{\frac{2}{3}} x\right\}+\left(\delta^{\frac{2}{3}} \lambda\right)\left\{\delta^{\frac{1}{3}} x\right\}$

$O\left(\epsilon^{\frac{4}{3}}\right)$

$\left[K_{0}-\lambda_{0} I\right]\left\{\delta^{\frac{4}{3}} x\right\}=-[\delta K-(\delta \lambda) I]\left\{\delta^{\frac{1}{3}} x\right\}+\left(\delta^{\frac{1}{3}} \lambda\right)\{\delta x\}+\left(\delta^{\frac{2}{3}} \lambda\right)\left\{\delta^{\frac{2}{3}} x\right\}$

$O\left(\epsilon^{\frac{5}{3}}\right)$

$\left[K_{0}-\lambda_{0} I\right]\{\delta x\}=-[\delta K-(\delta \lambda) I]\left\{\delta^{\frac{2}{3}} x\right\}+\left(\delta^{\frac{1}{3}} \lambda\right)\left\{\delta^{\frac{4}{3}} x\right\}+\left(\delta^{\frac{2}{3}} \lambda\right)\{\delta x\}+\left(\delta^{\frac{4}{3}} \lambda\right)\left\{\delta^{\frac{1}{3}} x\right\}$

$O\left(\epsilon^{2}\right)$

$\left[K_{0}-\lambda_{0} I\right]\left\{\delta^{2} x\right\}=-\left[\delta^{2} K-\delta^{2} \lambda I\right]\left\{x_{01}\right\}-[\delta K-\delta \lambda I]\{\delta x\}+\left(\delta^{\frac{1}{3}} \lambda\right)\left(\delta^{\frac{5}{3}} x\right)+$

$$
\left(\delta^{\frac{2}{3}} \lambda\right)\left(\delta^{\frac{4}{3}} x\right)+\left(\delta^{\frac{4}{3}} \lambda\right)\left\{\delta^{\frac{2}{3}} x\right\}+\left(\delta^{\frac{5}{3}} \lambda\right)\left\{\delta^{\frac{1}{3}} x\right\}
$$

$O\left(\epsilon^{\frac{7}{3}}\right)$

$\left[K_{0}-\lambda_{0} I\right]\left\{\delta^{\frac{7}{3}} x\right\}=-\left[\delta^{2} K-\delta^{2} \lambda I\right]\left\{\delta^{\frac{1}{3}} x\right\}-[\delta K-\delta \lambda I]\left\{\delta^{\frac{4}{3}} x\right\}+\left(\delta^{\frac{1}{3}} \lambda\right)\left(\delta^{2} x\right)+$

$$
\left(\delta^{\frac{2}{3}} \lambda\right)\left(\delta^{\frac{5}{3}} x\right)+\left(\delta^{\frac{4}{3}} \lambda\right)\{\delta x\}+\left(\delta^{\frac{5}{3}} \lambda\right)\left\{\delta^{\frac{2}{3}} x\right\}+\left(\delta^{\frac{7}{3}} \lambda\right)\left\{x_{01}\right\}
$$

$O\left(\epsilon^{\frac{8}{3}}\right)$

$\left[K_{0}-\lambda_{0} I\right]\left\{\delta^{\frac{8}{3}} x\right\}=-\left[\delta^{2} K-\delta^{2} \lambda I\right]\left\{\delta^{\frac{2}{3}}\right\}-[\delta K-\delta \lambda I]\left\{\delta^{\frac{5}{3}} x\right\}+\left(\delta^{\frac{1}{3}} \lambda\right)\left(\delta^{\frac{7}{3}} x\right)+\left(\delta^{\frac{2}{3}} \lambda\right)\left(\delta^{2} x\right)+$

$$
\left(\delta^{\frac{4}{3}} \lambda\right)\left\{\delta^{\frac{4}{3}} x\right\}+\left(\delta^{\frac{5}{3}} \lambda\right)\{\delta x\}+\left(\delta^{\frac{7}{3}} \lambda\right)\left\{\delta^{\frac{1}{3}} x\right\}+\left(\delta^{\frac{8}{3}} \lambda\right)\left\{x_{01}\right\}
$$

As in classical matrix perturbation, we will expand the eigenvector perturbation at each order as a linear combination of the basis vectors. If $m$ is the order of the perturbation, then: 


$$
\delta^{\frac{m}{3}} x=c \frac{m}{3}, 1 x_{01}+c \frac{m}{3}, 2 x_{02}+c \frac{m}{3}, 3 x_{03}
$$

In these equations, the unknowns are $c \frac{m}{3}, 1, \ldots, c \frac{m}{3}, 3, \delta^{\frac{m}{3}} \lambda$.

\section{Solution of the Problem}

The orthogonality relation yields

$$
y_{01}^{H}\left(x_{01}+\delta^{\frac{1}{3}} x+\delta^{\frac{2}{3}} x+\ldots\right)=\beta
$$

Equating the left and right hand side term by term, we get

$$
c_{\frac{m}{3}, 1}=0
$$

if $m \geq 1$.

At each order, we multiply successively by $y_{01}^{H}, y_{02}^{H}$ and $y_{03}^{H}$ to evaluate the eigenvalue and eigenvector perturbation. We have to solve sytems of equations of size three to obtain solutions to the perturbation coefficients at any order. We get three separate solutions emerging from the Jordan block eigenvector as we perturb the matrix. The lowest order solutions are

$$
O\left(\epsilon^{\frac{1}{3}}\right)
$$

$$
\delta^{\frac{1}{3}} \lambda=\left(\frac{y_{03}^{H}(\delta K) x_{01}}{y_{01}^{H} x_{01}}\right)^{\frac{1}{3}} e^{\frac{2 \pi i j}{3}}
$$

Here $1 \leq j \leq 3$ and $i=\sqrt{-1}$.

$$
\begin{gathered}
c_{\frac{1}{3}, 1}=\delta^{\frac{1}{3}} \lambda \\
c_{\frac{1}{3}, 2}=0 \\
c_{\frac{1}{3}, 3}=0
\end{gathered}
$$


Higher order perturbation coefficients can be obtained by applying this procedure. If we compute perturbation coefficients for $O\left(\epsilon^{2}\right)$, we have to write out equations for orders extending to the $O\left(\epsilon^{\frac{8}{3}}\right)$ problem. This is due to the observation made in Chapter 2 that to solve for the complete problem at any order $m$, we have to write out the equations for $O\left(\frac{m}{n}+\frac{n-1}{n}\right)$. Equations at different orders are coupled together and are solved to obtain the unknown perturbation coefficients. 


\section{Appendix C}

\section{Examples of Branch Point}

\section{Surfaces and their effects on}

\section{Localization}

We examine a few more examples of branch point surfaces and their effects on localization. We examine $Q\left(q_{i}, \epsilon_{1}\right)$ for a system of three pendula. We solve equations 3-1 and 3-2 for complex $\left(\lambda, \epsilon_{1}\right)$ given that $\epsilon_{2}$ is permitted to be real. We plot $Q\left(q_{i}, \epsilon_{1}\right)$ superposed on the square-root branch surface. We again see the correlation between the branch point curves and modal sensitivity.

We now plot the two root coalescence surfaces for the system of four pendula. We permit $\epsilon_{2}$ and $\epsilon_{3}$ to be real and solve for complex $\lambda$ and $\epsilon_{1}$. We obtain three surfaces, the real parts of which are plotted in figures B-1, B-2 and B-3. Note the complicated folds of these surfaces. On these surfaces, we would have two modes with appreciable sensitivity and with appreciable amplitude on two oscillators in complete analogy to the two pendulum problem. The other two modes are localized. Again three root coalescences would occur where these two root coalescences come close together. This three root coalescence forms a line in the space spanned by these real coordinates. Thus we have a surface of reduced dimension associated with the higher order coalescence. 


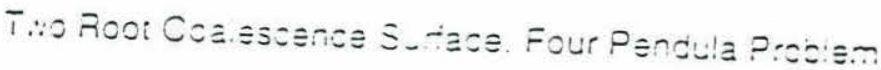

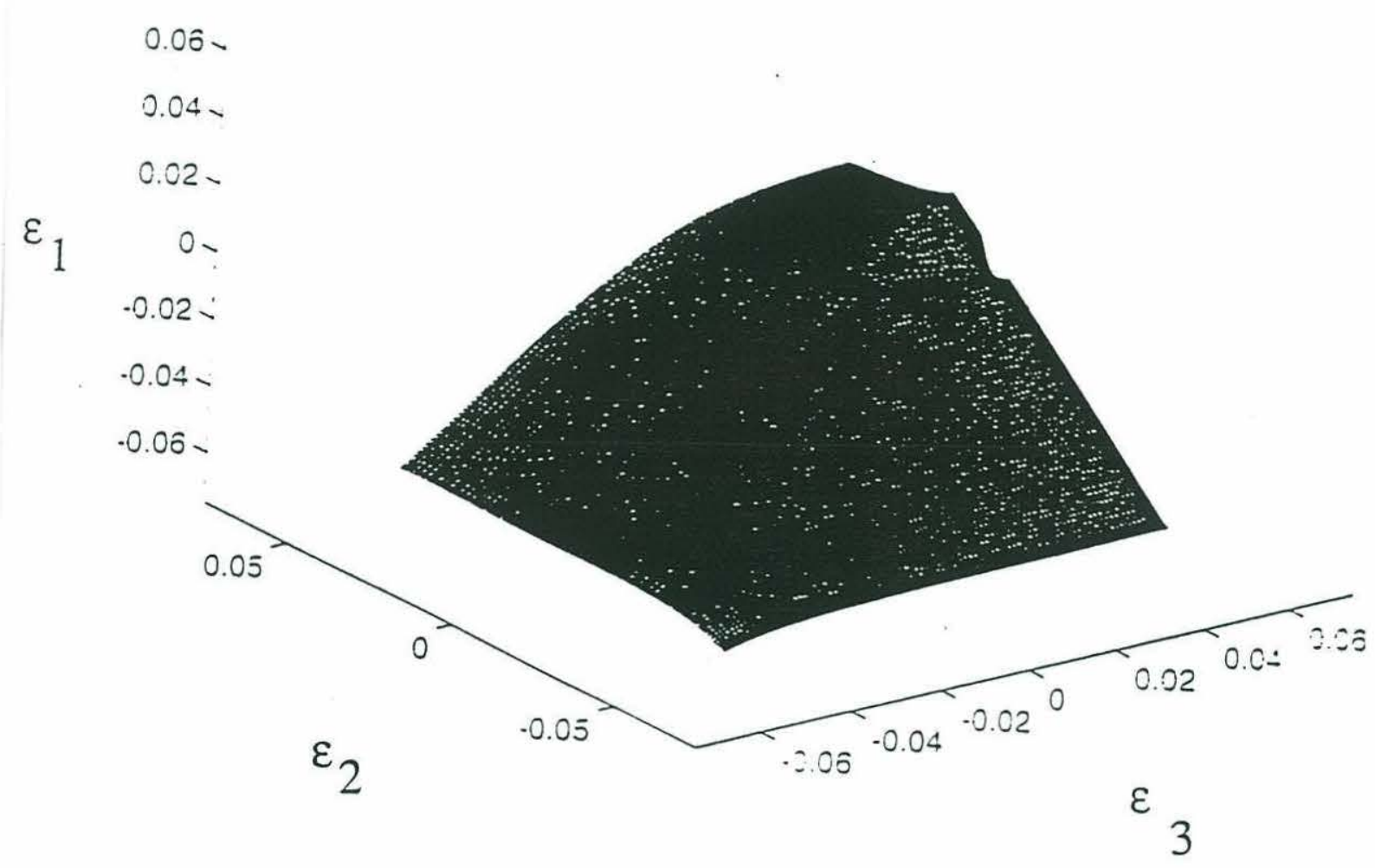

Figure C-2: Four Pendula System: Two Root Coalescence Surface Number 1 (Complex $\epsilon_{1}$ ) 


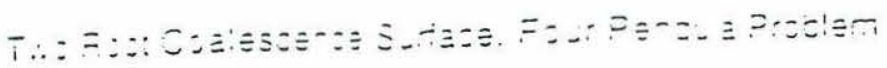

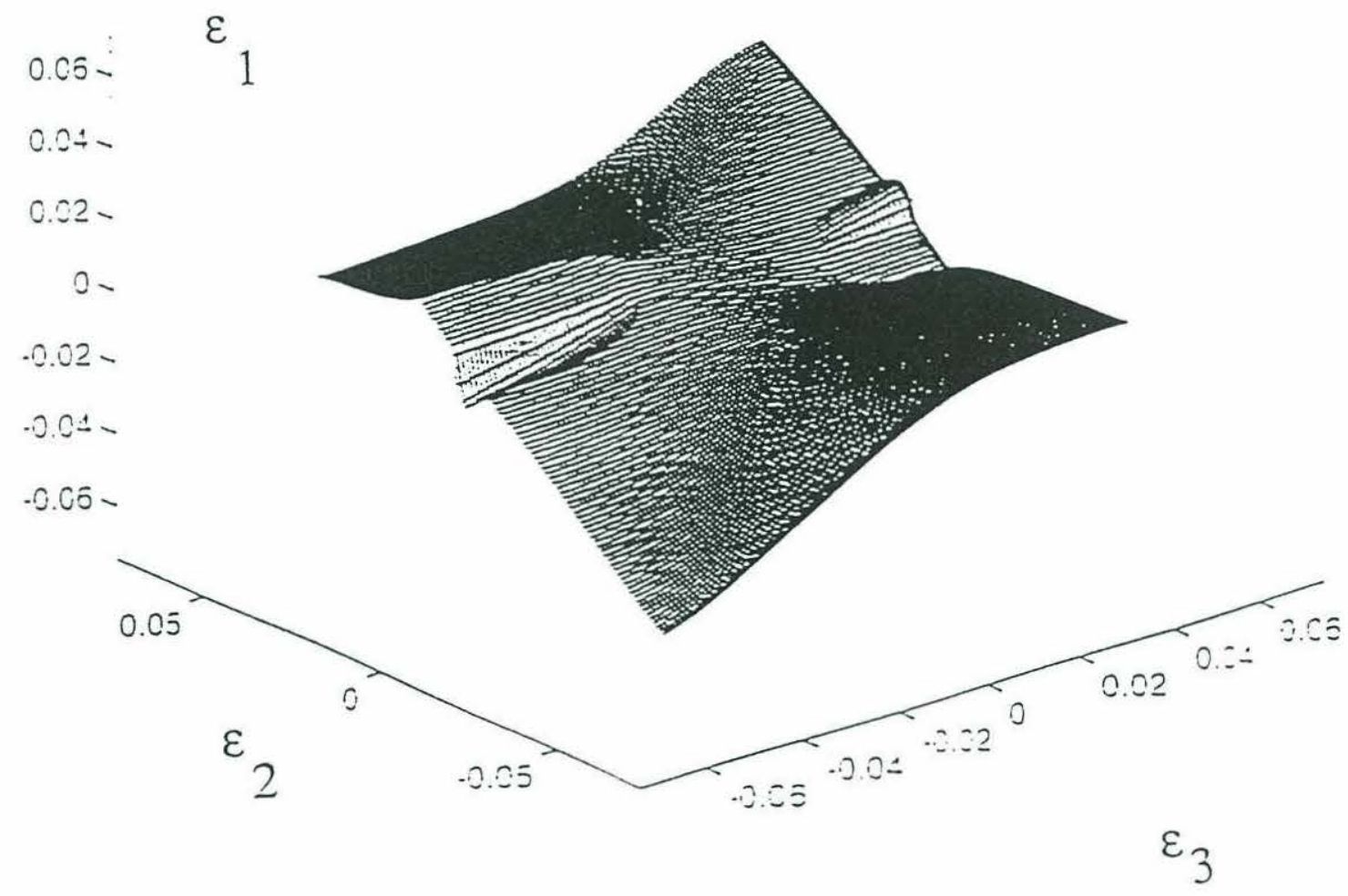

Figure C-3: Four Pendula System: Two Root Coalescezce Suriace Lumb:: 2 (Complex $\epsilon_{1}$ ) 
Two Root Cselescenze Suriace, Four Penzuie Projlen

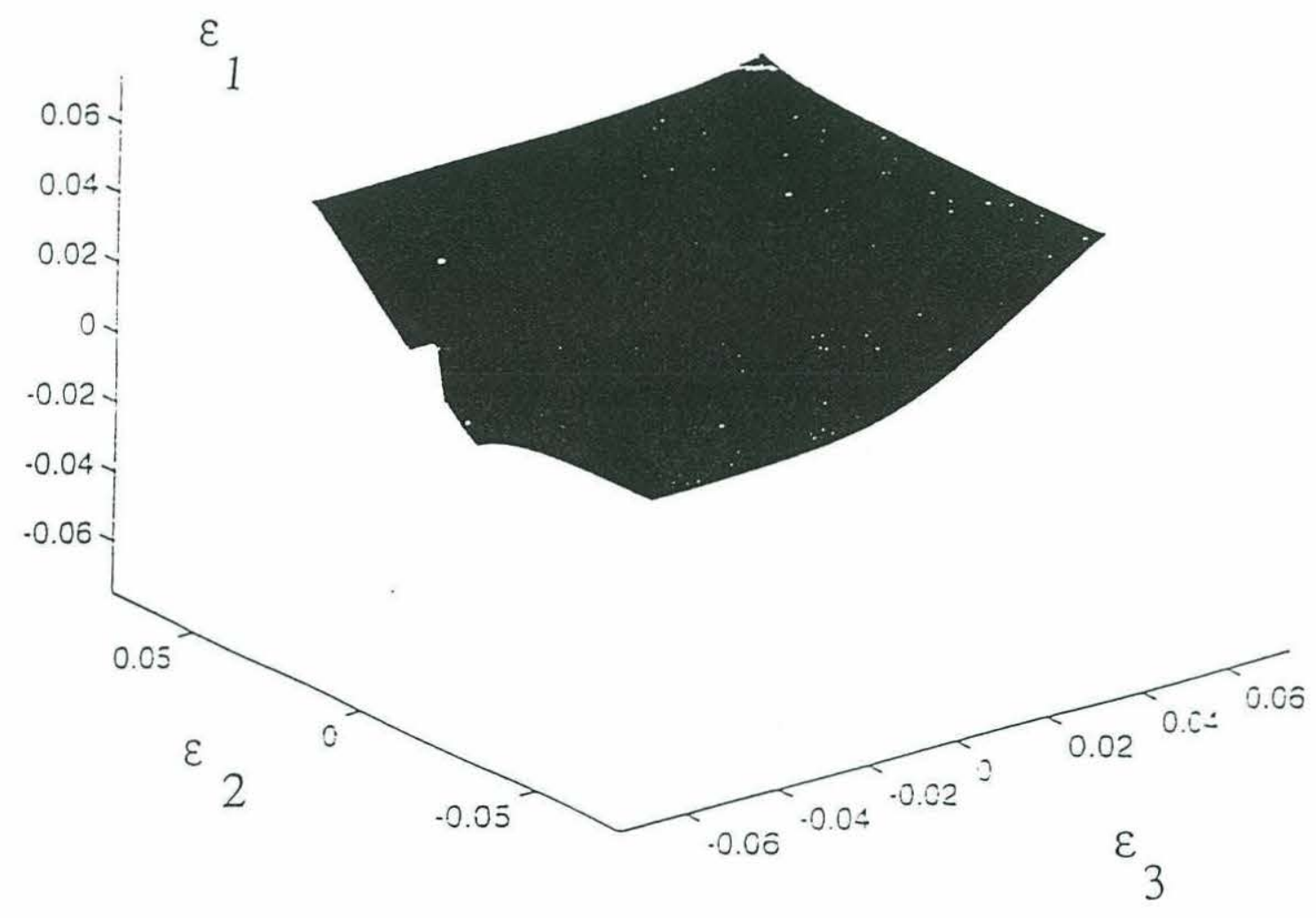

Figure C-!: Four Pendula System : Two Root Coalescence Surface Number 3 (Complex $\epsilon_{1}$ ) 


\section{Appendix D}

\section{Solution of the System of}

\section{Equations}

We now discuss the methods used to solve the system of equations. We note at the outset that optimal solutions using Lagrange multipliers are saddle point solutions. So we have not resorted to using minimization techniques like the conjugate gradient method (Rao [35]) to obtain the minimum of the objective function. There is no minimum, and only a saddle point exists at the solution. The only way to solve the problem is by actual solution of the conditions for existence of an optimal solution i.e. we solve the problem using nonlinear equation solution techniques rather than minimization techniques unless we use a penalty function approach(Rao [35]). The main solution method we used is the Gauss-Newton method with cubic-quadratic line-search (also called the Steepest Descent Method). Some of our calculations were verified using the NAG algorithm, the Fletcher-Powell method. We will briefly review this method also. For some of the trivial (smaller cases of three pendula) examples, we also verified the techniques using the Matlab command "fsolve", which utilized a combination of the Gauss-Newton (with cubic-quadratic line search) and the Levenberg-Marquadt method. The Levenberg-Marquadt solution is essentially a relative of the Fletcher-Powell method.

Consider a general system of nonlinear algebraic equations as given below. 


$$
f_{j}\left(x_{i}\right)=0
$$

Here $f_{i}$ is so that $1 \leq i \leq n$ and $1 \leq j \leq n$. We briefly compare the bare equations of the Steepest Descent and Levenberg-Marquadt Methods. These are all forms of explicit Jacobian Methods.

We shall frequently in this section, utilize the least-squares residue which is denoted as follows :

$$
g=\sum_{i=1}^{n}\left(f_{i}^{2}\right)
$$

\section{D.0.1 The General Form of Equations}

The General Equations of the Explicit Jacobian form are as follows

$$
x_{i+1}=x_{i}-H_{i} f_{i} t_{i}
$$

This general way of viewing these classes of solution methodologies has been advocated by Broyden [7]. Here $H_{i}$ is a matrix at the $i$ th iteration, $x_{i}$ is the $i$ the iteration, $\zeta_{i}$ is the function values at the $i$ th iteration, and $t_{i}$ is a fraction between 0 and 1 .

For the classical Newton method in $n$ dimensions, we have

$$
H_{i}=J_{i}^{-1}
$$

where $J_{i}$ is the Jacobian matrix associated with the system of equations D.1.

$$
t=1.0
$$

For the method of steepest descent, we have

$$
H_{i}=J_{i}^{T}
$$




$$
t_{i} \geq 0
$$

Note in the method of steepest descent, the gradient of the least squares residue $g$ is $2 J^{T} f$. And the factor 2 is absorbed in the $t_{i}$. For the Levenberg-Marquadt method, we have

$$
\begin{gathered}
H_{i}=\left(J^{T} J+\lambda_{i} I\right)^{-1} J^{T} \\
t_{i}=1.0
\end{gathered}
$$

We next provide some deeper explanation of the two methods.

\section{D.0.2 Method of Steepest Descent}

We now briefy prove that this is indeed the steepest descent direction following the development in Rao [35]. The rate of change of $g$ with respect to the step length $d s$ is given by

$$
\frac{d g}{d s}=\sum_{i=1}^{i=n} \nabla g^{T} \frac{d r}{d s}
$$

If $\mathbf{u}$ denotes the unit vector along the direction $d r$, we get

$$
d r=\mathbf{u} d s
$$

We then have

$$
\frac{d f}{d s}=\nabla f^{T} \mathbf{u}
$$

We write the gradient as an expansion in the components of the unit vectors $u_{i}$. We seek to select a set of unit vector components $u_{i}$ so that the descent direction is steepest while ensuring $\sum_{i=1}^{i=n} u_{i}^{2}=1$. We write the Lagrange function as 


$$
L\left(u_{i}, \lambda\right)=\frac{d g}{d s}+\lambda\left(1-\sum_{i=1}^{i=n} u_{i}^{2}\right)
$$

We set the derivatives of $L$ with respect to $u_{i}$ equal to zero. We obtain

$$
u_{i}=\frac{\frac{\partial g}{\partial x_{i}}}{|\nabla g|}
$$

and

$$
\lambda=\frac{|\nabla g|}{2}
$$

Thus the steepest descent direction is indeed the direction whose cosines correspond to that of the gradient.

There have been complaints raised against the convergence properties of the Gauss Newton Method(Rao [35]). See figure 4-1 for an example of situations where the convergence may be poor. We see that there is a tendency for the iterative scheme to move along directions which are in a zig-zag fashion rather than in a move along directions which are in a zig-zag fashion rather than in a path which goes directly to the solution.

The best search direction in a local sense is the direction along the gradient. However when we are attempting a solution in multiple dimensions, it is not necessary that the taking the entire Gauss-Newton step would be of advantage because the function may not necessarily be decreasing the entire step. It is necessary to determine how much of a step we should take in this direction. This is done by assuming the function to have a cubic or quadratic variation in this search direction(See Press et al. [33]) and then taking the step in such a fashion as to maximize the decrement of the function. The Gauss Newton method assures us linear convergence if we are sufficiently close to the solution. 


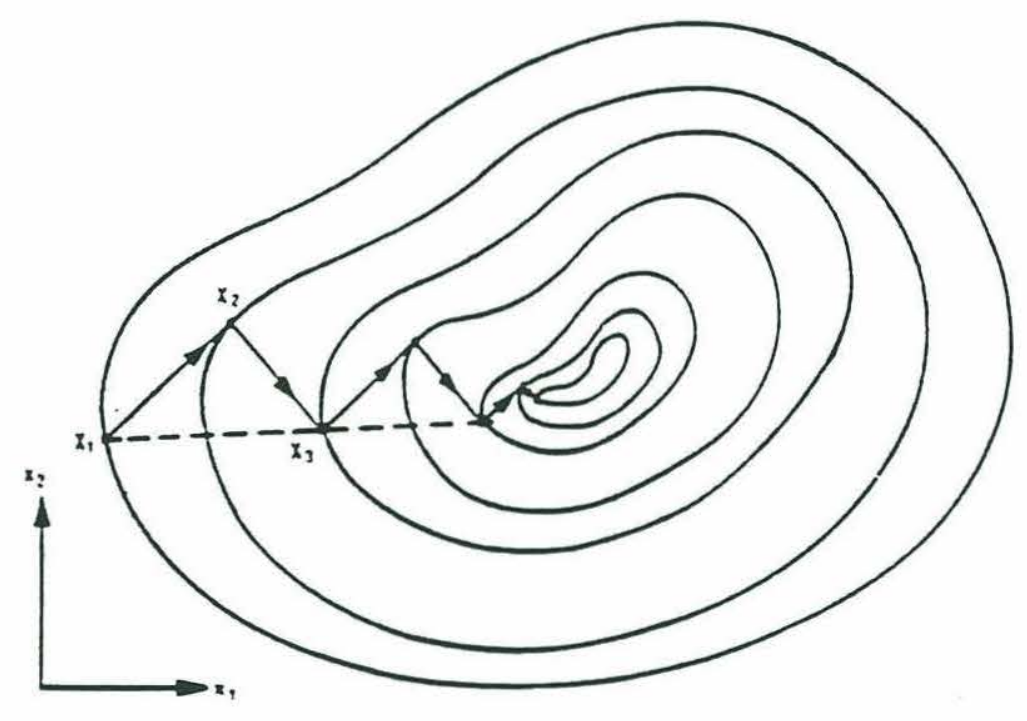

Figure D-1: Example Case where the Steepest Descent Method is inefficient 


\section{D.0.3 Model-Trust Region Approaches}

The Fletcher Powell Method and Levenberg Marquadt Methods are philosophically very different from the Steepest Descent Method and utilize a model trust region approach. We will very briefly describe the Fletcher Powell Method. Interested readers are referred to Broyden [7] or Powell [32] for greater details.

It has been numerically observed that the steepest descent method has linear convergence and is often slow in the neighborhood of the actual solution. But it is more effective further away from the actual solution. The Newton Raphson in multiple dimensions has a much smaller convergence zone but has a quadratic convergence close to the actual solution. The model trust region approach was designed to combine the best of both techniques.

If $\lambda_{i}$ is sufficiently large, we obtain the steepest descent step in the asymptotic limit while if $\lambda_{i}$ is sufficiently small, we get the Newton step. The Fletcher Powell and Levenberg-Marquadt method only differ in the way the Jacobian Matrix is obtained. The Levenberg Marquadt method relies on the use of analytical or finite difference formulations for the Jacobian. The Fletcher Powell Method analytically calculates the Jacobian only for the first iteration. Subsequent iterations use approximations which are as follow

$$
J^{k+1}=\frac{J^{k}+\alpha\left(\gamma^{k}-J^{k} \delta^{k}\left(\delta^{k}\right)^{T}\right)}{\left|\delta^{k}\right|^{2}}
$$

and the inverse of the Jacobian is

$$
H^{k+1}=H^{k}+\alpha \frac{\left(\delta^{k}-H^{k} \gamma^{k}\right) \delta^{k} H^{k}}{\alpha\left(\delta^{k} H^{k} \gamma^{k}\right)+(1-\alpha)\left\|\delta^{k}\right\|^{2}}
$$

The value of alpha is calculated by determining if

$$
\left|\delta^{k} H^{k} \gamma^{k}\right| \leq .1\left|\delta^{k}\right|^{2}
$$

where $\alpha=.8$ else $\alpha=1$. Also at each iteration, 


$$
\begin{gathered}
\delta_{i}^{k}=-H_{i, j} f_{j}\left(x^{k}\right) \\
\gamma_{i}^{k}=f_{i}\left(x^{k}+\delta^{k}\right)-f_{i}\left(x^{k}\right)
\end{gathered}
$$

This defines all the quantities in each iteration. The basis for these formulae are some theorems which guarantee that the error norm with respect to the actual Jacobian, using this sequence of formulae is going to decrease with each successive iteration. This approach avoids all the extra effort in computing the Jacobian at each time step in the Levenberg-Marquadt Method.

\section{D.0.4 Comparison Between the Different Methods}

According to R.S.Schnabel [36], numerical experimentation over the years has not indicated any consistently large differences between these methods. In our numerical experimentation too, we did not notice any really significant differences in the performances of these methods. 


\section{Bibliography}

[1] P. W. Anderson. Absence of diffusion in certain random lattices. Physical Review, 109:1492-1505, 1956.

[2] E. Balmes. High modal density, curve veering, localization: A different perspective on the structural response. Journal of Sound and Vibration, 161(2):358-363, 1993.

[3] C. M. Bender and S. A. Orzsag. Advanced Mathematical Methods for Engineers and Scientists. McGraw Hill Inc, 1978.

[4] R. D. Blevins. Formulae for Natural Frequency and Mode Shape. R. E. Krieger Pub. Co., 1971.

[5] R. E. Borland. The nature of electronic states in disordered one-dimensional systems. Proceedings of the Royal Society, A274:529-545, 1963.

[6] L. Brillouin. Wave Propagation in Periodic Structures. Dover Publications Inc., 1946.

[7] C. G. Broyden. Recent developments in solving nonlinear algebraic equations. In Phillip Rabinowitz, editor, Numerical Methods for Nonlinear Algebraic Equations, pages 61-72. Gordon and Breach Science Publishers, 1970.

[8] K. V. Bury. Statistical Models in Applied Science. John Wiley and Sons, 1975.

[9] P. J. Cornwell and O. O. Bendiksen. A numerical study of vibration localization in disordered cyclic structures. AIAA Journal, 89-1181-CP:199-208, 1989. 
[10] P.J. Cornwell and O. O. Bendiksen. Localization of vibrations in large space reflectors. AIAA Journal, 30:218-226, 1989.

[11] R. Courant and D. Hilbert. Methods of Mathematical Physics, Vol 1. Interscience Publishers Inc., 1953.

[12] P. Devillard, F. Dunlop, and B. Souillard. Localization of gravity waves on a channel with a random bottom. Journal of Fluid Mechanics, 186:521-537, 1988.

[13] Alvin W. Drake. Fundamentals of applied probability theory. Mc Graw Hill and Company, 1967.

[14] I. Gohberg, P.Lancaster, and L. Rodman. Matrix Polynomials. Academic Press Inc, 1982.

[15] G. S. Happawana, A. K. Bajaj, and O. D. I. Nwokah. A singular perturbation analysis of eigenvalue veering and modal sensitivity in perturbed linear systems. Journal of Sound and Vibration, 160(2):225-242, 1993.

[16] C. H. Hodges. Confinement of vibration by structural irregularity. Journal of Sound and Vibration, 82:411-424, 1982.

[17] C. H. Hodges and J. Woodhouse. Vibration isolation from irregularity in a nearly periodic structure: Theory and experiments. Journal of the Acoustic Society of America, 74(3):894-905, 1983.

[18] C. H. Hodges and J. Woodhouse. Theories of noise and vibration transmission in complex structures. Reports on Progress in Physics, 49:107-170, 1986.

[19] C. H. Hodges and J. Woodhouse. Confinement of vibration by one-dimensional disorder(1 \& 2). Journal of Sound and Vibration, 130(1 \& 2):237-269, 1989.

[20] G. J. Kissel. Mode Localization in Disordered Periodic Structures. PhD thesis, MIT, 1987.

[21] H. Lass and P. Gottlieb. Probability and Statistics. Addison-Wesley Publishing Company, 1971. 
[22] M. B. Levine and M. A. Salama. Experimental investigation for the mode localization phenomena. Technical Report JPL D-8767, Jet Propulsion Laboratory, Pasadena, California, 1991.

[23] A. Luongo. Mode localization by structural imperfections in one-dimensional continuous systems. Journal of Sound and Vibration, 155(2):249-273, 1991.

[24] J. N. Newman. Marine Hydrodynamics. MIT Press, 1977.

[25] N. C. Perkins and C. D. Mote. Comments on curve veering in eigenvalue problems. Journal of Sound and Vibration, 106(3):451-463, 1985.

[26] E. C. Pestel and F. A. Leckie . Matrix Methods in Elastomechanics. McGraw Hill, 1963.

[27] C. Pierre and E. H. Dowell . Localization of vibration by structural irregularity. Journal of Sound and Vibration, 114(3):549-564, 1987.

[28] C. Pierre. Mode localization and eigenvalue loci veering phenomena in disordered periodic structures. Journal of Sound and Vibration, 126(3):485-502, 1988.

[29] C. Pierre. Weak and strong localization in disordered structures: A statistical investigation. Journal of Sound and Vibration, 139(1):111-132, 1989.

[30] C. Pierre and P.D. Cha. Strong mode localization of vibration in nearly periodic disordered structures. AIAA Journal, 27:227-241, 1989.

[31] C. Pierre, D. M. Tang, and E. H. Dowell. Localized vibrations of disordered multi-span beams. AIAA Journal, 25(9):1249-1257, 1987.

[32] M. J. D. Powell. A fortran subroutine for solving nonlinear systems of equations. In Phillip Rabinowitz, editor, Numerical Methods for Nonlinear Algebraic Equations, pages 115-150. Gordon and Breach Science Publishers, 1970.

[33] William H. Press, Saul A. Teukolsky, William T. Vetterling, and Brian P. Flannery. Numerical Recipes In Fortran, The Art of Scientific Computing. Cambridge University Press, 1992. 
[34] G. Rajagopal. Mode localization for the case of masses on a string : Theory and experiment. In Proceedings of the 3rd ISOPE, San Francisco, volume 2, pages 426-433, 1992.

[35] S.S. Rao. Optimization Theory and Applications. Wiley Eastern and Company, 1978.

[36] R. B. Schnabel. Unconstrained optimization in 1981. In M.J.D.Powell, editor, Nonlinear Optimization, pages 3-16. Academic Press Inc., 1982.

[37] M. Seides. Mode localization in periodic structures. Master's thesis, Massachusetts Institute of Technology, 1988.

[38] A. A. Tjavaras. Dynamics of discrete nonlinear oscillator models for cables. Master's thesis, Massachusetts Institute of Technology, 1993.

[39] M. S. Triantafyllou and G. S. Triantafyllou. Frequency coalescence and modelocalization phenomena : a geometric theory. Journal of Sound and Vibration, 150:485-499, 1991.

[40] N. A. Valero and O. O. Bendiksen. Vibration characteristics of mistuned shrouded blade assemblies. American Society of Mechanical Engineers No. 85GT-115, 1985.

[41] J. H. Wilkinson. The Algebraic Eigenvalue Problem. Oxford University Press, 1965. 